\title{
Sprachliche Mittel des Humoristischen im Deutschen und im Polnischen
}


$\frac{3}{40}$ 
KATARZYNA SIKORSKA-BUJNOWICZ

\section{Sprachliche Mittel des Humoristischen im Deutschen und im Polnischen}

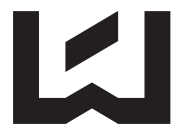

WYDAWNICTWO UNIWERSYTETU

ŁÓDZKIEGO

ŁÓDŹ 2013 
Katarzyna Sikorska-Bujnowicz - Universität Łódź, Fakultät für Philologie, Lehrstuhl für Deutsche und Angewandte Sprachwissenschaft Abteilung für Deutsche Sprachwissenschaft

90-114 Łódź, Sienkiewicza 21, Polen

\title{
REZENSENT
}

Lech Kolago

REDAKTION

Elżbieta Marciszewska-Kowalczyk

SATZ

AGENT PR

EINBANDGESTALTUNG

Barbara Grzejszczak

CC Copyright by Uniwersytet Łódzki, Łódź 2013

Alle Rechte vorbehalten

Wydane przez Wydawnictwo Uniwersytetu Łódzkiego

Wydanie I. W.06333.13.0.M

\section{ISBN 978-83-7969-049-7}

\author{
Wydawnictwo Uniwersytetu Łódzkiego \\ 90-131 Łódź, ul. Lindleya 8 \\ www.wydawnictwo.uni.lodz.pl \\ e-mail: ksiegarnia@uni.lodz.pl \\ tel. (42) 66558 63, faks (42) 6655862 \\ Druk i oprawa: Quick Druk
}




\section{INHALTSVERZEICHNIS}

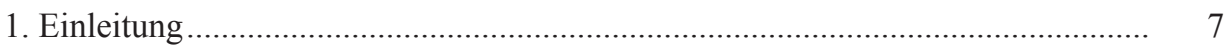

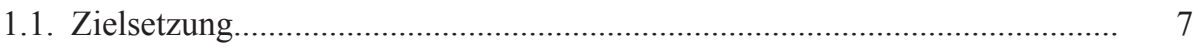

1.2. Methodologische Vorgehensweise und Korpus ......................................... 9

1.3. Zum Forschungsstand ........................................................................ 11

2. Lachen und Humor als Grundbegriffe der Humorforschung ............................. 13

3. Humortheorien. Kurzer Überblick .................................................................... 23

4. Witz als Grundeinheit der scherzhaften Kommunikation .................................. 29

4.1. Begriff des Witzes................................................................................... 29

4.2. Sachwitz und Sprachwitz....................................................................... $\quad 30$

4.3. Sketch und der konversationelle Humor ................................................... 32

4.4. Zum Ethnowitz ...................................................................................... $\quad 34$

5. Humor in den Medien ................................................................................... 39

5.1. Fernsehsendung als Element der Massenkommunikation............................. 39

6. Sprachliche Mittel des Humoristischen .............................................................. 45

6.1. Wortspiel als Mittel des Humoristischen ................................................ 46

6.2. Polysemie, Homonymie und Homophonie als Mittel des Humoristischen.... 55

6.3. Fremdwort als Mittel des Humoristischen..................................................... 64

7. Sprachliche Mittel des Humoristischen in „Was guckst du?““............................ 67

7.1. Struktur und Sprache von „Was guckst du?“ als Ethno-Comedy.................. 67

7.2. Ethnowitz in „Was guckst du? “............................................................ 83

8. Sprachliche Mittel des Humoristischen bei „Paranienormalni““ ......................... 101

8.1. Sprache und Formate von „Paranienormalni“ “........................................... 102

8.2. Quasivulgarismen als Mittel des Humoristischen im Polnischen ................. 108

8.3. Ethnowitz bei „Paranienormalni“ und „Ani Mru-Mru“ .............................. 121 
9. Intertextualität in den deutschen und polnischen Sketchtexten

10. Körpersprache als Element des Humoristischen............................................ 139

11. Schlussfolgerungen und Prognosen ........................................................... 147

Bibliographie und Quellenverzeichnis............................................................... 153

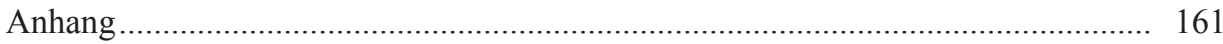

Liste der besprochenen Witze ...................................................................... 161

Verschriftungen der deutschen und polnischen Sketche ...................................... 167

Verzeichnis der besprochenen Sketchtexte ........................................................ 191 


\section{EINLEITUNG}

\subsection{Zielsetzung}

Das vorliegende Buch hat zum Ziel, die Ergebnisse meiner bisherigen Forschungen im Bereich des deutschen und des polnischen Humors zu präsentieren und die Frage zu beantworten, inwieweit sich die beiden Sprachsysteme im Bereich des Witzigen unterscheiden und wo sie doch ihre Gemeinsamkeiten haben.

Ich gehe davon aus, dass es trotz der sprachlichen Unterschiede doch Gemeinsamkeiten beim Ausdruck des Humoristischen gibt, was wiederum davon zeugen kann, dass der Humor an sich, obwohl er als etwas Angeborenes verstanden und definiert werden kann, auch als eine objektive, allgemeingültige Größe anzusehen ist, was auf zwei mit dem Wort Humor zusammenhängende Begriffe - den Sinn für Humor und die Gefühlslage zurückgeht.

„Was guckst du?" ist ein besonders für die Zwecke dieser Analyse geeignetes Programm, weil es als Ganzes als Ethno-Comedy einzustufen ist, was selten der Fall ist, wenn es um Sendungen dieser Art geht. Es werden hier Formate angeboten, die ganze Serien bilden und für die die meisterhafte Art und Weise, auf die der Humor sowohl sprachlich als auch außersprachlich ausgedrückt wird, charakteristisch ist. Im Rahmen der Sendung wird nicht nur mit dem Wortwörtlichen, sondern auch mit dem abwechslungsreichen Inventar an der polysemen Lexik aus verschiedenen Bereichen gespielt.

Im Vergleich zu dem sogenannten typisch deutschen Sinn für Humor, der für solche Komiker wie Markus Maria Profitlich (,Mensch, Markus!“) zu nennen ist oder im Vergleich zu anderen deutschen Comedy-Programmen, wie „Alt und durchgeknallt“ oder „Die dreisten drei“, wo mit dem Wortwörtlichen, dem Direkten gespielt wird, ist „Was guckst du?“ ein besonderes Phänomen innerhalb der deutschen Medienlandschaft des Unterhaltungsfernsehens. Es ist dieses erste Programm, das als die so genannte Ethno-Comedy auf dem deutschen Fernsehmarkt erschienen ist. Zum ersten Mal wurden in den neunziger Jahren so direkt die Probleme der Einwanderer in Deutschland besprochen. Bei der Analyse fällt auf, dass verschiedene Schwierigkeiten, auf die die Ausländer in Deutschland sto- 
ßen, alle Nationen im gleichen Maße betreffen. Nicht weniger wichtig ist dabei die Tatsache, dass alles Problematische zwar im Zerrspiegel, aber mit viel Humor und Sympathie präsentiert wird.

Auf eine äußerst interessante Art und Weise wird von den Autoren der ethnische situative Kontext mit dem dementsprechend formulierten sprachlichen Kontext zusammengestellt, so dass die sich daraus ergebenden Szenen an die deutsche Realität gebunden sind, die für die Einwanderer aus ganz anderen Kulturkreisen völlig fremd und nicht selten auch unverständlich zu sein scheint. Die Konfrontation mit dem voll von Problemen aller Art gefüllten Alltag wird von den Autoren der Texte aus „Was guckst du?" als eine unerschöpfte Quelle der zu besprechenden Situationen und Schwierigkeiten betrachtet. Nicht ohne Bedeutung ist hier der Moderator selbst - Kaya Yanar, in Deutschland geborener Mann türkisch-arabischer Abstammung, dem als dem ersten ausländischen Komiker gelungen ist, sich mit seinen Ideen einer im deutschen Fernsehen auszustrahlenden Ethno-Comedy durchzusetzen und mit jeder neuen Folge an Popularität zu gewinnen, nicht nur beim deutschen Publikum.

Nach jeder neuen Serie konnte festgestellt werden, wie konsequent sich die Autoren daran halten, auf scharfe Kritik der Deutschen zu verzichten und sich darauf zu konzentrieren, alle auszulachenden Nationen gleich zu behandeln. In den Sketchen werden Vertreter anderer Kulturkreise, samt Landsleuten des Moderators im Zerrspiegel dargestellt. Das ist eben das Charakteristische - daran erkennt man eben das Objektive in dieser Sendung, nämlich die Tatsache, dass nicht nur die Deutschen als Zielscheibe zu verstehen sind, sondern dass man versucht, die Vertreter aller für die Zwecke der Sendung in die einzelnen Szenen miteinbezogenen Nationen gleich zu betrachten, was auf den gemeinsamen Nenner zurückgeht - Probleme eines nach Deutschland kommenden Ausländers, der mit allem Möglichen konfrontiert wird, was auf ihn in seiner neuen Heimat zukommt, unabhängig davon, aus welchem Land und welchem Kulturkreis er kommt.

Im Rahmen der Sendung wird der situative Kontext mit dem sprachlichen so ergänzt, dass sich daraus meisterhafte humoristische Szenen ergeben, die sich als Material für diese Analyse eignen, die den Charakter einer konfrontativen Studie hat. Man spielt hier einerseits mit der Mehrdeutigkeit auf der lexikalischen Ebene und andererseits auch mit der fehlenden Kenntnis der deutschen Sprache auf drei Ebenen - der der Aussprache, der Grammatik und der Syntax.

Unter den polnischen Kabarettgruppen, die nur einen ethnischen Humor anbieten, gibt es keine, dafür aber gibt es in dieser Szene Gruppen, die das Problem der auf Schwierigkeiten in Polen als ihrer neuen Heimat stoßenden Ausländer ansprechen - unter anderem „Paranienormalni““ und „Ani Mru-Mru“. Für die Zwe- 
cke der vergleichenden Analyse eignen sich besonders gut die Texte der Gruppe „Paranienormalni“" auch aus diesem Grund, dass hier ähnliche Formate wie in „Was guckst du?", auch in Form der ganzen Serien angeboten werden.

Auf der sprachlichen Ebene konnten gleiche Phänomene erkannt werden, d.h. Probleme mit der polysemen Lexik, fehlende Kenntnisse der Sprachen, sei es des Polnischen, des Französischen oder des Englischen, so wie bei „Was guckst du?“, wobei es in der deutschen Sendung in erster Linie um die Schwierigkeiten mit der deutschen Sprache geht und auch um die Darstellung der Sprachen aus dem arabischen Kulturkreis. Nicht weniger oft wird von den Autoren auch mit den fehlerhaften Wortformen und Wendungen gespielt, so dass im Endeffekt auch über die sprachlichen Fehler gelacht werden kann.

\subsection{Methodologische Vorgehensweise und Korpus}

Die im Rahmen dieser Abhandlung präsentierten und besprochenen sprachlichen und außersprachlichen Erscheinungen basieren auf der Analyse des Forschungsmaterials, das sich aus den von mir verschrifteten Texten aus der deutschen Fernsehsendung „Was guckst du?“ (eigene Aufnahmen des Programms aus den Jahren 1995-2000, insgesamt 116 Sketchtexte $^{1}$ ) und den von der polnischen Gruppe „Paranienormalni“ und „Ani Mru-Mru“( (insgesamt 33 Texte) und aus den deutschen und polnischen Witzen, die den Internetseiten entstammen (47 Witze) zusammensetzt. Es wurden aus dieser Menge insgesamt 80 Texte als Paradebeispiele aus beiden Sprachen ausgewählt und ihre Analyse in dem Buch gezeigt und besprochen.

Aus Platzgründen habe ich als Belege nur die repräsentativsten deutschen und polnischen Texte ausgewählt, um folgende Fragen beantworten zu können:

- Welche sprachlichen Mittel zum Ausdruck des Humoristischen können in beiden Sprachen genannt werden?

- Gibt es, unabhängig von dem Sprachsystem, ein universelles sprachliches Mittel zum Ausdruck der witzigen Inhalte?

- Was ist nur für eines der beiden Systeme charakteristisch?

Ich habe eine These aufgestellt, dass im Polnischen stärker emotional geprägte witzige Texte entstehen als im Deutschen, wobei aber nicht vergessen werden

${ }^{1}$ Die aus eigener Aufnahmensammlung der Fernsehsendung „Was guckst du?“ aus den Jahren 1995-2000 stammenden Szenen wurden von mir benannt und gruppiert. 
darf, dass die Texte in „Was guckst du?" zwar auf Deutsch geschrieben wurden, aber in Verbindung mit den Elementen außersprachlicher Natur aus einem völlig anderen Kulturkreis präsentiert werden, was der Sendung im Endeffekt ein besonderes Kolorit verleiht.

Ich bin davon ausgegangen, dass die deutschen und polnischen witzigen Texte unter dem Aspekt der sprachlichen Mittel des Humoristischen konfrontativ analysiert werden können. Zwischen den beiden Sprachen sind vor allem Unterschiede im Gebrauch fehlerhafter Formen festzustellen, denn im Falle von „Was guckst du?" geht es zusätzlich um die sogenannten Ausländer-Deutsch-Varianten, was dann auch bestimmte Fehler impliziert, vor allem phonetische, grammatische und syntaktische. Es betrifft auch den Gebrauch der polysemen Lexeme. Wegen dieses festzustellenden und zu analysierenden Phänomens habe ich alle Texte in ihrer Originalform gelassen, ohne sie korrigiert zu haben. Ich habe angenommen, dass es in den polnischen Texten um Fehler einer anderen Art geht - um solche, die den übertriebenen Gebrauch von Elementen der Jugendsprache und der Umgangssprache betreffen und dabei auch - um die Vulgarismen. Aus der Analyse ging hervor, dass die von mir für die Zwecke dieser Abhandlung untersuchten Texte von „Paranienormalni“ besonders oft eine homophone Form eines polnischen Vulgarismus enthalten, die ich Quasivulgarismus genannt habe. Interessant ist dabei die Funktion der Vulgarismen als Mittel des Humoristischen im Polnischen, die im Vergleich zu den deutschen Texten mich eindeutig feststellen liess, dass das Gegenwartspolnisch als Sprache der witzigen Texte ohne diese obszönen Elemente heutzutage nicht auskommen kann, denn diese sind schon zum festen Bestandteil der polnischen Lexik geworden, man darf sogar behaupten, dass sie an Schärfe verloren haben, weil sie heutzutage eher als Mittel zum Ausdruck der starken Emotionen gebraucht werden oder einfach als Floskeln, ohne die es kaum Gespräche unter Gleichgesinnten gibt.

Es fällt dabei doch auf, dass in beiden Sprachen vor allem mit drei sprachlichen Erscheinungen gespielt wird - mit der Polysemie, der Homonymie und der Homophonie.

Die Art des angebotenen Humors setzt eher die Kenntnisse der umgangssprachlichen Varianten der beiden Sprachen voraus, nicht die der Literatursprache, die für die Zwecke des Humoristischen alle möglichen stilistischen Mittel zur Verfügung stellt. Auf die genaue Besprechung der stilistischen Mittel wurde demzufolge verzichtet. 


\subsection{Zum Forschungsstand}

Jede Forschung im Bereich des Witzigen beruft sich auf die Ursprünge der Gelotologie, die auf Freud [1993, 1985, 1982] und Bergson [1972] zurückgehen. Im Rahmen der neuesten Humorforschungen gibt es zwar zahlreiche Abhandlungen über den Humor als Phänomen in jeder Sprache, vor allem im Englischen, Französischen [Dżereń-Głowacka 2008, 2009; Kwiatkowska, Stanecka 2012; Dynel 2009; Szeflińska-Baran 2012] und im Polnischen [Buttler 1961, S. 7, 2001; Bystroń 1960; Brzozowska 2004; Sikorska-Bujnowicz 2012c,] betreffen oder aber das Deutsche als Sprache des Humoristischen besprechen, was vor allem auf Kotthoff zurückgeht, die das deutsche Sprachsystem unter verschiedenen Aspekten des Humoristischen untersucht, d.h. u.a. in Bezug auf die Gendersprachen [1986, 1988, 1992, 1998, 2000, 2002a, 2006] und den konversationellen Humor [1994, S. 73-99, 1997a, 1997b, 2004a, 2006, 2007]. In ihren Arbeiten beschäftigt sie sich auch mit den mit dem Verstehen des Witzes zusammenhängenden Erscheinungen [1997c, 1998b] und mit der Intertextualität innerhalb der Witze [2000, 2002b]. Nicht weniger wichtig ist für Kotthoff auch das Problem des Humors in den Medien [2002a, 2004a, 2004b, 2004c] und die mit dem Ethnowitz zusammenhängenden Fragen (2009).

Im Rahmen der polnischen Humorforschung muss vor allem auf Buttler [1961, 2001] und Bystroń [1960] als Gründer der polnischen Humorforschung hingewiesen werden, die sich für das Phänomen der sprachlichen Witze interessiert haben und auf die man sich bis heute in den Abhandlungen über das polnische Sprachsystem als Quelle des Humoristischen beruft. Sie haben den Grundstein zur Humorforschung im Polnischen gelegt.

Da es keine konfrontativen Arbeiten Deutsch vs. Polnisch gab, die das Problem der sprachlichen Mittel des Humoristischen in beiden Sprachen am Beispiel der Sketchtexte besprechen, habe ich mich entschieden, zwei Sprachsysteme unter dem Aspekt des Humoristischen zu untersuchen - das Gegenwartspolnische und das Gegenwartsdeutsche [Sikorska 2009, 2009b, S. 161-167, Sikorska-Bujnowicz 2010, S. 69-78, 2011a, S. 134-141, 2011b, S. 69-78, 2012a, S. 55-62, 2012b, S.17-24, 2012c, S. 153-159]. Bisher hat man noch nicht versucht, eben die beiden genannten Sprachen als Mittel zum Ausdruck der witzigen Texte zu vergleichen. Das Beobachten der Comedyszenen in Deutschland und Polen liess mich zu dem Schluss kommen, dass trotz der sprachlichen Unterschiede doch Gemeinsamkeiten beim Einsatz der Sprache als Mittel des Humoristischen sowohl im Deutschen als auch im Polnischen festgestellt werden sollen. 
Bei der Definition des Humors an sich konnte ich mich auf die Arbeiten von Bremmer und Roodenburg [1999] stützen, die den Humor als ein sprachliches Phänomen betrachten, wobei die psycho-sozialen Aspekte vor allem bei Plessner [1970, 1982, S. 201-387, 1983] zum Thema der Abhandlung wurden.

Als Mittel des Witzigen verstehe ich auch das Nonverbale, das alles Gesagte bzw. Geschriebene nur meisterhaft unterstreichen kann und dem eine besondere Aufgabe zugeschrieben steht. Man darf dabei aber nicht nur dieses Phänomen im Auge behalten, sondern es als einen untrennbaren Teil des Humoristischen verstehen, was in zahlreichen Arbeiten zu diesem Thema zu sehen ist, z.B. bei Matschnig ${ }^{2}$.

Im kommenden Kapitel wird auf die Auffassung des Humors, den Begriff des Lächelns und des Lachens und ihre Funktionen eingegangen, die in verschiedenen Disziplinen zum Forschungsthema geworden sind und von verschiedenem Gesichtspunkt aus betrachtet werden.

Für die Zwecke dieser Analyse schliesse ich mich der Auffassung der Psychologen und Soziologen an, dass der Humor als Charaktereigenschaft an sich eine objektive Größe ist, die jedem Menschen individuell zugeschrieben steht. Der Humor ist ein angeborenes Merkmal, wobei die Interpretationen des gesagten bzw. geschriebenen Humoristischen schon etwas Subjektives darstellen, was im Endeffekt eben auf dieses Angeborene zurückgeht. Dieses Phänomen kann ein Beweis dafür sein, dass der Sinn für Humor und das Verstehen und Interpretieren aller witzigen sprachlichen und außersprachlichen Inhalte eine untrennbare, zusammenhängende Einheit bilden und als solche erst das richtige Verstehen der Witze ermöglichen.

${ }^{2}$ Mit den Problemen der nonverbalen Kommunikation und ihrem Einfluss auf die zwischenmenschlichen Relationen, sei es im Alltag, im Beruf, in der Beziehung usw. beschäftigt sich u.a. Matschnig. Vgl. dazu www.matschnig.com; http://matschnig.com/home/medien-und-presse/fachberichte/, darunter u.a. Matschnig [2007a, 2007b, 2011a, 2011b, 2012]. 


\section{LACHEN UND HUMOR ALS GRUNDBEGRIFFE DER HUMORFORSCHUNG}

Im Rahmen der Kommunikation soll auf zwei Arten dieses Prozesses hingewiesen werden - die ernste und nicht-ernste Kommunikation, die als Scherzkommunikation bezeichnet wird. An der Bezeichnung selbst kann schon erkannt werden, dass mit dem Scherz alles, was nicht ernst ist, zusammenhängt. Mit dem Begriff Scherzkommunikation hängen noch weitere Termini zusammen, d.h. Spaß und Humor ${ }^{3}$, und sie sind als Unterbegriffe der Scherzkommunikation zu verstehen. Es muss dabei betont werden, dass der Humor und die Witzigkeit wegen ihrer Bedeutung innerhalb der nicht-ernsten Kommunikation nicht separat betrachtet werden dürfen, denn sie bestimmen ihren Charakter und tragen dazu bei, dass der scherzhafte Kommunikationsprozess als eine Einheit verstanden werden soll. Kotthoff [1998a, S. 146] weist auf Folgendes hin

Witziges ist im Alltag nicht auf die Gattung der Standardwitze beschränkt, wenngleich der schriftlich präsentierte Witz in der Linguistik die am meisten behandelte Textsorte repräsentiert (Marfurt 1977, Raskin 1985, Attardo/Chabanne 1992). Komik lässt sich aber fast überall entdecken und konversationell erzeugen. Komik ist im Unterschied zu Witzigkeit nicht auf eine Pointe angewiesen.

und betont somit die Rolle der mündlichen Sprache bei der Erzeugung des Komischen, was nicht weniger wichtig als die Verschriftung des Witzigen ist, was spä-

\footnotetext{
${ }^{3}$ Spaß, der; -es, Späße [älter: Spasso < ital. spasso = Zeitvertreib, Vergnügen, zu: spassa$\mathrm{re}=$ zerstreuen, unterhalten, über das Vlat. zu lat. expassum, 2. Part. von: expandere $=$ auseinander spannen, ausbreiten, aus: ex= aus u. pandere= ausspannen, ausbreiten, ausspreizen]: 1. ausgelassen-scherzhafte, lustige Äußerung, Handlung o.̈̈., die auf Heiterkeit, Gelächter abzielt; Scherz 2. $<$ o.Pl. $>$ Freude, Vergnügen, das man an einem bestimmten Tun hat, Duden - Deutsches Universalwörterbuch 2001; Hu|mor, der; -s, -e $<$ Pl. selten $>$ [älter engl. humour = literarische Stilgattung des Komischen, eigtl. $=$ Stimmung, Laune $<$ afrz. humour $<$ lat. (h)umores $=($ Temperament $\mathrm{u}$. Charakter bestimmende) Körpersäfte, zu: (h)umor= Feuchtigkeit, Flüssigkeit]: 1. <o.Pl.> Fähigkeit u. Bereitschaft, der Unzulänglichkeit der Welt u. der Menschen, den Schwierigkeiten u. Missgeschicken des Alltags mit heiterer Gelassenheit zu begegnen [...] 2. sprachliche, künstlerische o. ̈̈. Äußerung einer von Humor (1) bestimmten Geisteshaltung, Wesensart [...] 3. <0.Pl.> gute Laune, fröhliche Stimmung [...] Duden - Deutsches Universalwörterbuch 2001
} 
ter als Ausgangspunkt für die sprachwissenschaftlichen Analysen zur Verfügung steht. Alle Begriffe des Humoristischen werden aus Sicht verschiedener Disziplinen ${ }^{4}$ definiert, wobei auf andere Aspekte hingewiesen wird.

Innerhalb der Kulturkunde sind der Witz und der Humor als solche Erscheinungen zu besprechen, für deren Verständnis von dem Rezipienten, sei es von einem Zuschauer bzw. einem Zuhörer, das kulturelle Wissen verlangt wird, was vor allem in Bezug auf ethnische Witze und ihre Interpretationsmöglichkeiten unbestritten ist.

Im Rahmen der Soziologie wird darauf gezeigt, dass alle mit dem Humor zusammenhängenden Erscheinungen der sprachlichen und außersprachlichen Natur gleichzeitig die Gruppengrenzen innerhalb der Gesellschaft bestimmen. Auf diese Weise kann festgestellt werden, mit wem und worüber gelacht wird und - was noch wichtiger ist - gelacht werden kann. Der Witz bekommt auf diese Weise gleichzeitig eine besondere Aufgabe zugeschrieben. Er soll in alles eingreifen, was sich in der Gesellschaft abspielt und in allen Bereichen kritisch wirken.

Die Voraussetzung für das Witzverstehen und für die richtige, von dem $\mathrm{Zu}$ schauer bzw. Zuhörer erwartete Interpretation ist das Vorhandensein seiner Humorkompetenz [Marhenke 2003], die der Sprachkompetenz gleichgestellt wird. Die individuellen und kulturellen Unterschiede zwischen den an der Scherzkommunikation beteiligten Gesprächspartnern üben einen bestimmten Einfluss auf die Humorperformanz aus. Demzufolge wird dabei in erster Linie darauf geachtet, wer worüber lacht, d.h. ob die Zugehörigkeit zu einer bestimmten sozialen Gruppe die Art der Witze, ihre Struktur ${ }^{5}$, d.h. Thema, Motiv, Zielscheibe und Pointe und das damit verbundene Soziolekt auch die Sprache der Witze beeinflussen oder nicht.

Gewöhnlich wird darauf hingedeutet, dass der Humor als Haltung und Gefühlslage zu verstehen sei und als etwas dem Menschen Nicht-Angeborenes betrachtet wird. Der Humor hängt eher mit der Intention des Gesprächspartners dem anderen Sprecher gegenüber zusammen. Der Humor als Haltung und der als Gefühlslage hängen aber mit dem Begriff des Persönlichkeitsmerkmals zusammen. Den Sinn für Humor kann man nicht erlernen, er ist etwas Angeborenes, etwas Objektives. Diese Auffassung ist für die Psychologie charakteristisch. Dieser These schließe ich mich an.

Das Lachen wird auch als ein soziales Phänomen aufgefasst. Über die soziale Funktion des Lachens schreibt zuerst Bergson [1972], nach dem das Lachen eine soziale Funktion hat. Es ist eine Erscheinung, die für eine Gruppe von Menschen

${ }^{4}$ Vgl. dazu Humorforschung. Wo ist der Witz?, htpp://www.zeit.de/2007/31/humorforschung (Stand vom 14.06.2010).

${ }^{5}$ Dazu hat sich zum ersten Mal Raskin [1985] geäußert, der auf die Semantik der witzigen Texte und deren Struktur hinweist. 
charakteristisch ist. Er geht davon aus, dass die Menschen nur faule Gewohnheitstiere seien, die versuchen, sich in ihrer nicht perfekten Welt zurechtzufinden. Diese Eigenschaft verstößt aber gegen das Interesse der Gesellschaft, in der bestimmte Regeln gelten, wie z.B. die Fähigkeit der Gesellschaftsmitglieder, sich an alle möglichen Umstände anzupassen und ihr soziales Engagement. Um es aufrechtzuerhalten, braucht man nach Bergson so was wie das Lachen, das eben als eine soziale Geste zu verstehen sei. Demzufolge sind auch negativ gekennzeichnete Schadenfreude oder das Auslachen gar nicht als asozial zu verstehen. Ihnen wird eine andere, regulierende Funktion zugeschrieben, denn das Lachen sei ein Erziehungsmittel.

Neben der von Bergson genannten sozialen Funktion gibt es eine weitere, die dem Humor zugeschrieben wird - die informative oder die appellative Funktion. Dies ist darauf zurückzuführen, dass über alles und jeden gelacht werden kann. Erst die Antwort auf die Frage, ob über etwas oder jemanden gelacht werden darf, ist eine kulturspezifische Sache.

Für Plesner [1970, 1982, S. 201- 387, 1983, S. 136-217] ist das Lachen an sich als ein Ausdruck der körperlichen Reaktion auf etwas, was sprachlich nicht beantwortet werden kann, zu verstehen. Dieses Fremde, was den Menschen zum Lachen bringt, weicht von den Normen verschiedener Art ab und somit stellt das Interessante und eben das Normwidrige dar, was seinen Ausdruck in den witzigen Texten wiederfindet. Die Wahrnehmung der nicht nur eine Interpretation zulassenden Witze geht darauf zurück, an welche Normen der Rezipient gebunden ist, an welche Regeln er sich hält und was für ihn komisch ist. Demzufolge kann es nichts Objektives sein, denn die Rezeption bedeutet doch immer etwas Subjektives. Die kulturelle Identität, die Einstellung zu allem Weltlichen und Heiligen, die von dem Rezipienten bevorzugte Lebensweise usw. - das alles beeinflusst nicht nur die Wahrnehmung der außersprachlichen Realität, sondern auch die der sprachlichen. Die Tatsache, wofür und wogegen der Zuschauer oder Zuhörer ist, wen oder was er wichtig findet, welche Prinzipien in seinem Leben an erster Stelle stehen, bewirkt auch seine Reaktionen auf die witzigen Texte, die laut Definition lustig sein sollen und die Aufgabe haben, die Rezipienten zuerst zu lächeln zu provozieren und im Endeffekt zum Lachen zu bringen.

Mit dem Lachen innerhalb einer sozialen Gruppe können unterschiedliche Funktionen assoziiert werden. In erster Linie dient es der Vermittlung von Emotionen, sowohl der positiven, wie z. B. Zuneigung, Sympathie, als auch der negativen, wie die Ablehnung. Nicht weniger wichtig scheint dabei noch eine weitere Funktion des Lachens zu sein - die den Stress abbauende und die Erregung mildernde. Mit dem Lachen wird das Unangenehme an einem bereits geführten Gespräch überdeckt und die ganze Situation somit auch lockerer gemacht. Das 
gemeinsame Lachen unter Menschen bringt sie näher und hat demzufolge die Funktion „eines »sozialen Klebstoffs"“[Titze 2009b, S. 11-39]. Andererseits aber dient das Lachen auch als ,eine kommunikative Waffe“[Ebenda], die zu dem Zweck eingesetzt wird, andere bloßzustellen, zu demütigen und auf diese Weise "geschickt" zu verletzen.

Das Lächeln und das Lachen wurden im Rahmen der Ethologie ${ }^{6}$, also der Verhaltensforschung, als Ausdruck des Negativen, sogar der Aggression interpretiert, was sich auf das Zeigen der Zähne und die Vergleiche der menschlichen Welt mit der Tierwelt zurückzuführen lässt.

Der Begriff des Humors wurde im Laufe der Zeit verschieden erläutert. In DUDEN $^{7}$ (Großes Wörterbuch der deutschen Sprache und Das Herkunftswörterbuch) wird das Lexem Humor einerseits mit der menschlichen Gabe, dem Temperament des Individuums und andererseits - mit der sprachlichen Äußerung von Humor gleichgesetzt. Mit Humor hängt noch eine weitere - fachliche - Bedeutungsvariante aus dem medizinischen Bereich zusammen, die auf die antike Temperamentenlehre zurückgeht. Man geht in dieser Linie davon aus, dass die menschliche Gesundheit von der Balance zwischen den vier Körpersäften, lat. humores genannt, abhängig ist. Diese vier Körperflüssigkeiten - sanguis 'Blut', phlegma 'Schleim', melas cholé 'schwarze Gallenflüssigkeit' und cholé 'gelbe Gallenflüssigkeit' bilden eine Mischung, die für jeden Menschen typisch ist und

${ }^{6}$ Etho|lo|gie, die; - [lat. Ethologia $=$ Sitten- od. Charakterdarstellung $<$ griech. ethología $]$ : Wissenschaft vom Verhalten der Tiere u. des Menschen; Verhaltensforschung. 2000 Dudenverlag; Vgl. Eibl-Eibesfeldt [1967, S. 140], zit. in Titze [2009b].

${ }^{7} \mathrm{Hu} \mid$ mor, der; $-\mathrm{s}$, $-\mathrm{e}<\mathrm{Pl}$. selten $>$ [älter engl. humour = literarische Stilgattung des Komischen, eigtl. $=$ Stimmung, Laune $<$ afrz. humour $<$ lat. (h)umores $=$ (Temperament $\mathrm{u}$. Charakter bestimmende) Körpersäfte, zu: (h)umor= Feuchtigkeit, Flüssigkeit]: 1. $<0 . P 1 .>$ Gabe eines Menschen, der Unzulänglichkeit der Welt u. der Menschen, den Schwierigkeiten u. Missgeschicken des Alltags mit heiterer Gelassenheit zu begegnen: er hat, besitzt einen unverwüstlichen, goldenen H.; etw. mit H. nehmen, tragen; ein Mensch ohne H.; zwischen sehr tiefem Ernst, überwallender Verzagtheit und bitterem H. (Geissler, Wunschhütlein 15); er hat keinen H. (reagiert sehr leicht verärgert, ist nicht in der Lage, etw. gelassen auf-, hinzunehmen); für seine Unverschämtheiten habe ich keinen H. [mehr] (sie missfallen mir zu sehr); er hat keinen Sinn für H. (ihm fehlt der Humor, er ist humorlos); R du hast, er hat [ja vielleicht] H.! (was denkst du dir, denkt er sich eigentlich!); Spr H. ist, wenn man trotzdem lacht. 2. sprachliche, künstlerische o.ä. Äußerung einer von Humor (1) bestimmten Geisteshaltung, Wesensart: der rheinische, der Kölner H.; gezeichneter H. (Humor in der Kunstform der Karikatur o.Ä.); schwarzer (das Grauen, Grauenhafte einbeziehender) H.; Ein tolles Stück (=Theaterstück). Angefüllt mit schwarzen -en und brillanten Pointen (MM 27.1. 68, S. 68). 3. <o.Pl.> gute Laune, fröhliche Stimmung: den H. [nicht] verlieren, behalten; Typen wie dieser... bringen mich um jeglichen H. (Frisch, Stiller 295). 4. Laune, Stimmung, Gefühlslage: Dies gab mir den allerschlimmsten H., besonders da ich den Übungsort selbst ganz unerträglich fand (Goethe, Dichtung u. Wahrheit 4).C 2000 Dudenverlag Hu|mor, der; -s, -es [hu'mo:re:s; lat. (h)umor, 1Humor] (Med. selten): Körpersaft. 2000 Dudenverlag; vgl. dazu auch DUDEN. Das Herkunfstwörterbuch, 295 
nur als seine Eigenschaft verstanden werden kann. Man hat davon die bis heute im Rahmen der Psychologie geläufigen Bezeichnungen des menschlichen Temperaments abgeleitet, d.h.

- Sanguiniker als 'der heitere, aktive Mensch',

- Phlegmatiker als 'der passive, schwerfällige Mensch',

- Melancholiker als 'der traurige, nachdenkliche Mensch' und

- Choleriker als 'der reizbare und erregbare Mensch' definiert.

Der Sinn für Humor wurde dem Sanguiniker zugeschrieben, wegen des für diesen Typ charakteristischen heißen Bluts, mit dem früher die positive Einstellung und die Lebensfreude assoziiert wurden. Dabei tauchte noch ein weiteres Wort auf, nämlich humorig, das man den Individuen als ihre Charaktereigenschaft [+launig] und [+mit Humor] zugeschrieben hat. Die gelassene Art, alles locker und mit Humor zu sehen, bewirkte die Wahrnehmung einer solchen Person als jemanden mit Sinn für Humor. Man ist dabei darauf gekommen, dass ein Mensch mit diesem Persönlichkeitsmerkmal [+mit Sinn für Humor] auch weitere zu nennende, nur für ihn charakteristische Fähigkeiten besitzt, d.h. [+optimistisch] , $[+ \text { mit Abstand betrachtend }]^{8},[+$ alles mit viel Humor wahrnehmend], [+kognitiv flexibel], [+sozial kompetent], [+kommunikativ] und [+mit dem Lachen eigene Kräfte aktivierend] [Titze 2009a, S. 20-25].

In der Literatur zur Gelotologie wird darauf hingewiesen, dass die Wirkung des Lachens sowohl sozial als auch asozial sein kann, was als die an sich widersprüchliche Funktion des Lachens verstanden wird. Wenn gelacht wird, können sich die Gesprächspartner entweder miteinander verbunden oder aber aus der Gruppe ausgeschlossen fühlen, wenn über sie Witze erzählt werden. Im ersteren Fall liegt die Kohäsion, in dem weiteren - die Exklusion vor. Die Geletologen schreiben dem Lächeln und dem Lachen eine besondere Bedeutung zu:

Das lächelnde Gesicht ist ein »Spielgesicht«. Es stellt ein universales Signal für Freundlichkeit, Kooperationsbereitschaft, Zustimmung und Freude dar.[Ebenda]

Die beiden können nämlich als Mittel der Therapie eingesetzt werden, was auf ihre Entspannungswirkung zurückgeht. Man nennt auch Eigenschaften eines hu-

${ }^{8}$ Psychologen sprechen hier über die Resilienz, die als psychische Widerstandsfähigkeit des Menschen zu verstehen sei. [vgl. dazu auch Titze 2009a]. Der heitere Mensch ist imstande, selbst dank seiner Charakterzüge, die notwendige Distanz zu allen ihn belastenden Lebenssituationen herzustellen, indem von ihm deren Bedeutung relativiert wird. Das hat zur Folge, dass Ereignisse und Situationen besser ertragen werden können. 
morvollen Menschen, der andere nie auslacht, sich als Element einer Ganzeit versteht, die Mitmenschen mit seinem Optimismus ansteckt, Gefühle und Vernunft gleichsetzt und weiß, dass das Ernsthafte und das Traurige ihre komplementären Gegenerscheinungen haben, d.h. das Nicht-Ernsthafte und die Freude.

Die Humortheorien im Rahmen der Psychologie gehen auf den bereits mehrmals genannten Freud zurück, der eine Witztheorie und eine Humortheorie als zwei scharf voneinander abgegrenzte Theorien sieht. Freud versteht den Humor als eine sehr positive psychische Leistung, die als eine Abwehrform sogar bei der Bewältigung des Alltags und aller mit ihm zusammenhängenden Probleme helfen soll und demzufolge als positiv zu verstehen ist. Der Witz wird von ihm als Mittel zur Befriedigung sexueller und aggressiver Triebtendenzen gesehen. Freud nennt drei Bereiche, in denen diese Triebe befriedigt werden müssen und wo somit der jeweilige Aufwand erspart bleibt

- feindliche und aggressive, wegen der sozialen Normen zu unterdrückende Impulse

- verbotene, tabuisierte Themen

- unschuldige Spieltriebe.

Freud geht davon aus, dass der Witz und die Komik mit den menschlichen Trieben zusammenhängen. Sie haben eine Entspannungsfunktion. Die den Menschen störenden Tabus können von ihm selbst in einem Witz durchbrochen und besiegt werden. Das Lustige kann aufgrund der tabuisierten Inhalte entstehen, so dass der Erzählende und der Zuhörer auf diese Weise einerseits das Tabu brechen, andererseits - es entkrampfen, in Frage stellen und zum Schluss noch darüber lachen.

Bei Bremmer und Roodenburg [1999] wird der Humor neutral definiert, d.h. als eine Botschaft, die mit Hilfe verschiedener, dem Menschen zur Verfügung stehender Mittel formuliert wird und das Lächeln oder das Lachen hervorruft. Unter diesen Mitteln verstehen sie das Sprechen selbst, das Schreiben, die Bilder oder die Musik. Sie betonen dabei, dass diese Botschaft gezielt formuliert wird, also nicht zufällig zustande gebracht wird. Es gibt aber keine Möglichkeit, eine universelle Humor- und Lachtheorie zu bilden, was auf die Verschiedenheit der anders an sich konstruierten und anderen Normen folgenden Kulturen zurückzuführen ist. Bei Bremmer und Roodenburg ist von dem ontologischen Charakter des Humors die Rede, weil er überall gültig ist und keine Geschichte hat.

Der Humor kann aber auch als eine Widerspiegelung der tieferen kulturell verankerten Vorstellungen und Ideen definiert werden, was vor allem die Ethnowitze betrifft. 
Driessen [1999] unterstreicht eine besondere Funktion der Sprache im Rahmen des Humoristischen, denn eine die Witze verschiedener Art produzierende, erzählende und interpretierende Nation verfügt über ihre eigenen Mittel, mit denen derselbe komische Inhalt versteckt und demzufolge auch - entschlüsselt werden kann. Im Zusammenhang damit soll man an dieser Stelle auch das Problem der Übersetzbarkeit von Witzen ansprechen [Dimova 2008, S. 7-20], was vor allem auf die Spezifik des jeweiligen lexikalischen Systems zurückgeht. Erst die Kenntnis der Sprache, in der ein witziger Text verfasst ist, mit allen ihren Nuancen, Doppeldeutigkeiten und übertragenen Bedeutungen, ermöglicht den Rezipienten eine von ihnen erwartete Interpretation vorzunehmen und zum Schluss noch darüber zu lachen. Driessen vertritt die Meinung, dass eben das Verstehen der Sprache des Witzes mit dem Einblick in die Kultur der jeweiligen Nation zusammenhängt und diese Interpretation dann auch garantiert. Das richtige, also von dem Rezipienten erhoffte Finden, Entschlüsseln und Interpretieren der versteckten humoristischen Inhalte verlangt jedoch die relativ guten Sprachkenntnisse, nicht nur auf der lexikalischen, sondern auch auf der morphosyntaktischen Ebene. Das von den Autoren geschickt für die Zwecke des Humoristischen eingesetzte Spiel mit der Sprache verlangt auch das Sprachgefühl auf der anderen Seite - auf der der Zuhörer oder der Zuschauer als Partner innerhalb der scherzhaften Kommunikation.

Interessant zu sein scheint in diesem Zusammenhang die Auffassung des Humors innerhalb der Soziologie, wo dieser als menschliche Fähigkeit, alles Komische wahrzunehmen, begriffen wird und einen universellen Charakter hat [Berger 1998]. Bergers Theorie gleicht der von Plessner, wo die Rede davon ist, dass der Humor von verschiedenen Nationen und zu verschiedenen Zeiten auf eine ganz andere Art und Weise aufgefasst wird.

Für die Zwecke dieser Arbeit ist jedoch von größter Bedeutung, auf die Sichtweise der Linguistik hinzuweisen, wo betont wird, dass die Sprache mit ihrem ganzen Inventar von lexikalischen und stilistischen Mitteln zur Erzeugung des Humoristischen dient.

Im Rahmen der Kommunikationsforschung wird in Bezug auf das Humoristische vor allem auf die Verbindung von Produktion und Rezeption hingewiesen. Bei Freud [1985] wurden die Funktionen der Witze genannt, die mit der Befriedigung der Aggression, des Triebs zum Sprechen über Tabus und des einfachen Spieltriebs zusammenhängen. Wichtig ist dabei die Tatsache, dass alles zum Thema des Witzes werden kann. Freuds Auffassung des Humors und der Witze wird viele Jahre später um weitere Elemente ergänzt, d.h. um das Gegensätzliche, wo darauf gezeigt wird, dass die Wahrnehmung der Gegensätze zum Entstehen des Komischen führen kann. 
Für das Verstehen des Witzes und seine beabsichtigte Wirkung sind vier Bereiche von Bedeutung, d.h sprachliches Wissen, kulturelles Wissen, das Common Sense-Wissen und das Kontextwissen [Cui 2008].

Die vorhandenen bzw. fehlenden Sprachkenntnisse haben einen großen Einfluss auf das Verstehen des sprachlichen Kontextes innerhalb eines witzigen Textes, wobei das genannte Common Sense-Wissen auf die außersprachliche Realität bezogen ist und somit kulturelles Wissen impliziert. Die genannten Bereiche sind vor allem im Falle der Nationen- bzw. Ethnowitze von Bedeutung. Darunter werden Witze von Nationen und über Nationen verstanden, in denen als Zielscheibe die Nationalität, die Religion bzw. die regionale Zugehörigkeit fungieren. Der Ausgangspunkt für diesen Typ der witzigen Texte sind Stereotypen und Klischees. Bestimmten Nationen werden in den Witzen ${ }^{9}$ konkrete negative Merkmale zugeschrieben, z.B. Polen werden als Autodiebe präsentiert:

\section{(1) Wie lautet die Werbung eines polnischen Reisebüros in Deutsch- land? \\ Kommen Sie nach Polen, Ihr Auto ist schon da!}

Türken sind schlechte Nachbarn der Deutschen

(2) Kauft ein Türke eine Villa, sein Nachbar auch. So ging es weiter mit anderen Sachen. Dann sagt der Türke: "Ich habe es besser." "Warum?", fragt der deutsche Nachbar, der Arzt ist. "Ich habe einen Arzt als Nachbar und Sie - einen Türken!",

Schotten dagegen sind geizig

(3) Der Schotte kommt in die Bäckerei und bittet den Bäcker "Bitte ein Stück Brot! Wickeln Sie es bitte in die Zeitung von heute ein!"

und Russen - alkoholsüchtig

${ }^{9}$ Die Witztexte entstammen verschiedenen Sammlungen, darunter sind folgende zu nennen: www.witz-des-tages.de, www.dein-witz.de, Kunschmann [2003], Drews [2005], Hallstatt [2002], Millowitsch [1970]. In dieser Arbeit werden in den weiteren Kapiteln die Ethnowitze aus „Was guckst du?" und die von den polnischen Gruppen „Paranienormalni“" und „Ani Mru-Mru“ analysiert. 
(4) Ein Russe bestellt immer fünf Schnaps gleichzeitig im Restaurant. Er trinkt für seine vier gefallenen Kameraden mit. Es soll eine Abmachung sein, dass der Überlebende für die anderen mittrinken müsse und er sei der einzige Überlebende. Eines Abends bestellt er nur vier Schnaps.

"Ich trinke nur für meine gefallenen Freunde. Ich selbst bin Antialkoholiker geworden."

Auch die regionale Zugehörigkeit kann zum Thema der Witze werden. Innerhalb der deutschen Komik werden u.a. Ostfriesen als die Dummen dargestellt

(5) Die Frau eines Ostfriesen bekam Drillinge. Was macht der Ostfriese? Er sucht nach den anderen beiden Männern.

Es kann aber vorkommen, dass sie doch als die Klügeren präsentiert werden, wenn ihnen die Bewohner einer anderen Region gegenübergestellt werden, z.B. Schwaben

(6) Was machen die Ostfriesen bei Ebbe? Sie verkaufen Land an die Schwaben.

Zur Zielscheibe der witzigen Texte werden auch verschiedene Berufsgruppen, z.B. Polizisten ${ }^{10}$

(7) Ein Polizist kommt in eine Buchhandlung und will einen Akkordeon kaufen.

Die Verkäuferin erwidert : „Es tut mir leid, aber es ist kein Akkordeon, es ist der Heizkörper!"

(8) Warum gehen die Polizisten nie alleine?

Der eine kann lesen, der andere schreiben.

oder Ärzte

${ }^{10}$ Diese Witze gibt es auch auf Polnisch, was auf ihren internationalen Charakter hinweist. 
(9) Der Arzt beruhigt seinen Patienten vor der Operation. „Machen Sie sich keine Sorgen. Ich habe diesen Eingriff schon hundertmal vorgenommen!"

Der Patient sagt "Da haben Sie mich aber beruhigt, Herr Doktor!" Der Arzt erwidert "Na ja, einmal muss es doch klappen!"

Es hat sich auch eine besonders große Menge von Witzen über Blondinen entwickelt, sowohl im Deutschen als auch im Polnischen.

(10) Jada dwie blondynki tramwajem. W pewnym momencie jedna z nich pyta "Dojade do dworca? " Na co druga: "A ja?"11

(11) Was sagt die Blondine, wenn sie erfährt, dass sie schwanger ist? "Sind Sie sicher, Herr Doktor, dass es meins ist?"

Es kommt aber manchmal auch zur Vermischung der Motive in den Witzen, wo das Ethnische miteinbezogen wird, z.B.

\section{(12) Ein Chinese kommt mit zwei Blondinen aus der Bäckerei. Warum? Er wollte zwei Blödchen!}

In diesem Falle liegt ein Witz vor, der gleichzeitig als ein Ethno- und ein Blondinenwitz einzustufen ist. Im Hintergrund wird angenommen, dass ein Chinese Probleme mit dem Aussprechen des deutschen [r] haben muss und anstelle von [r] nur ein [l] gebraucht, so dass es im Endeffekt zum Missverständnis kommt. Dazu wird das Wort Blödchen mit einer blöden Blondine assoziiert, denn die beiden Lexeme Brötchen und Blödchen sind als Pseudohomophone zu verstehen.

In den Witzen dieser Art wird nicht mit versteckten Inhalten gespielt. Man erwartet von dem Rezipienten nicht, dass er zwischen den Zeilen liest und das Verborgene findet und analysiert. Die Ethnowitze an sich sind direkte Beschreibungen der jeweiligen Nation, in denen nicht mit der Sprache gespielt wird, sondern darauf gezeigt wird, was an einer bestimmten Nation als störend empfunden wird.

${ }^{11}$ Zwei Blondinen fahren mit der Straßenbahn. Die eine fragt „Komme ich mit der Straßenbahn zum Bahnhof?“ Gleich fragt die zweite besorgt „Und ich?“ 


\section{HUMORTHEORIEN. KURZER ÜBERBLICK}

Alle Erscheinungen, die mit dem Humor und der Witzigkeit zusammenhängen, sind seit Langem das Thema der linguistischen Forschungen. Man kann die Humortheorien [Drösser 1998; Kotthoff 1996a] nach dem Aspekt einteilen, der im Vordergrund der Untersuchungen steht. In erster Linie sollen die Abhandlungen im Rahmen der Bewusstseinspsychologie und der Psychoanalyse (Freud) genannt werden, in denen man versucht hat, eine Definition des Humors zu formulieren. Es wird davon ausgegangen, dass das Lachen als menschliche Eigenschaft mit seinem Nervensystem zusammenhängt, d.h. jeder Mensch als Individuum hat die Fähigkeit und dank der Sprache auch die Möglichkeit, das Humoristische zu erzeugen. Nach den Annahmen der Instinkt- und Evolutionspsychologie wird der in den Witzen versteckte lustige Inhalt individuell interpretiert.

Die Überlegenheitstheorie (Bergson) schreibt dem Humor eine eher negative Funktion zu, d.h. der Humor soll als eine Bestrafung und Erniedrigung unsozialer Personen verstanden werden. Dabei wird auf diese Weise auch das Gefühl der Sympathie ausgedrückt.

Die Vertreter der Inkongruenztheorien (Kant, Schopenhauer, Koestler) betonen, dass das gleichzeitige Erscheinen von verschiedenen scheinbar unpassenden Elementen in Form von Ideen oder den von dem üblichen Verhalten abweichenden Situationen zum Entstehen des Humoristischen führt. Die genannten Kontexte lassen mindesten zwei Interpretationen zu, demzufolge ist die Bisoziation möglich, weil die humoristischen Elemente inkongruent sind.

Im Zentrum des Interesses der Überraschungstheorie ${ }^{12}$ steht das plötzliche und unerwartete Ende - die für einen witzigen Text charakteristische Pointe, die die Rezipienten überrascht, so dass sie zum Schluss über den gehörten bzw. gelesenen Witz zu lachen anfangen.

Wenn die Witze erzählt werden, dann geschieht es mit der Absicht, dass zum Schluss gelacht werden soll, wobei das Humoristische und das Lachen aus der Konfrontation der positiven und negativen Emotionen der Erzähler und des Rezi-

\footnotetext{
${ }^{12}$ Diese Auffassung vertrat schon Descartes [1649].
} 
pienten resultieren. Diese Auffassung ist für die Ambivalenztheorie charakteristisch, wobei man auf die Inkongruenz der Elemente keinen Wert legt.

Der Humor kann auch als Mittel der Entspannung definiert werden, dessen Funktion darin besteht, die Belastung und Anspannung zu lösen. Die so genannte Ventilfunktion des Humors steht im Mittelpunkt der Entlastungstheorien. ${ }^{13}$

Die Hauptgedanken der Konfigurationstheorien ${ }^{14}$ bilden scheinbar unpassende, inkongruente Elemente, die plötzlich eine völlig zusammenhängende witzige Einheit mit einem überraschenden Ende bilden. Diese im Endeffekt lustige Form wird als Konfiguration bezeichnet.

In der differenziellen Theorie ${ }^{15}$ wird zwischen kognitiven, konativen und affektiven ${ }^{16}$ Theorien unterschieden. Für die kognitiven ${ }^{17}$ Theorien gilt der Begriff der Inkongruenz, wobei es in den konativen ${ }^{18}$ und affektiven um das Trieb- und Antriebsgeschehen geht.

Zum Schluss sollen noch die Einfaktorentheorien erwähnt werden, zu denen die affektiven und die kognitiven gehören. Nach ihnen können nur diese Witze untersucht werden, in denen das Gefühl der Überlegenheit vermittelt wird. Dabei gibt es noch die sog. Mehrfaktorentheorien, zu deren Vertretern Freud [1905], Eysenck [1972] und Koestler [1964] gehören.

In den 80er und 90er Jahren des 20.Jahrhunderts sind zwei grundlegende, bis heute geltende und führende Theorien des verbalen Humors entstanden - die Semantische Skript Theorie des Humors (SSTH) von Raskin [1985] und die Generelle/Allgemeine Theorie des Verbalen Humors (GTVH) von Attardo/Raskin [1991].

Der Grundbegriff der Semantischen Skripttheorie des Humors (Semantic Script Theory of Humour) von Raskin [1985] ist das Skript. Darunter ist ein semantisches Feld um die Wörter und Begriffe herum zu verstehen, das aus semantischen Informationen besteht, die die mit den in einem Witz gebrauchten Wörtern verbundenen Assoziationen determinieren. Nach Raskin ist das Skript einer kognitiven Struktur ähnlich, die für jeden Muttersprachler im Falle eines konkreten

${ }^{13}$ Schon im 19. Jh hat man es betont [Spencer 1860].

${ }^{14}$ Als Vertreter dieser Theorien wird Maier [1932] genannt.

${ }^{15} \mathrm{Im}$ Rahmen dieser Forschungsrichtung wird Eysenck erwähnt.

${ }^{16}$ af|fek|tiv <Adj.> [spätlat. affectivus] (Psych.): gefühlsbetont, durch Affekte gekennzeichnet.

${ }^{17}$ ko|gni|tiv <Adj.> (bes. Psych., Päd.): das Wahrnehmen, Denken, Erkennen betreffend; erkenntnismäßig: -e Fähigkeiten; -es Lernen; In den Schulungskursen ... liegt der Schwerpunkt auf der Förderung -en Verhaltens, auf Lesen, Rechnen und Schreiben (NZZ 21.8. 83, S. 23); dass der Mensch k., affektiv und psychomotorisch besonders dann zu fördern ist, wenn man seine Funktionen seziert und einzeln trainiert (Zeit 29.3.85, S. 41); k. orientierte Sprachwissenschaft; k. orientierte Therapien. 2000 Dudenverlag;

${ }^{18}$ ko|na|tiv <Adj.> (Psych.): strebend, antriebhaft. 2000 Dudenverlag 
Wortes charakteristisch ist [Raskin 1985]. Beim Entschlüsseln der Bedeutungsvarianten eines Wortes wird gleichzeitig ein bestimmtes Skript abgerufen. Es wird davon ausgegangen, dass der Witz einerseits mit zwei Skripts kompatibel sein muss, die andererseits aber gleichzeitig in einer Opposition zueinander stehen. Raskin hat fünf am meisten in den witzigen Texten zu findende Skriptoppositionen genannt: „good/bad, life/ death, non-obscene/obscene, money/no-money, high stature/low stature“"[Ebenda, S. 113-114, 127; zit. nach: Cui 2008].

Man kann die genannten Thesen der beiden Theorien mit folgenden Beispielen belegen

(13) Zwei Blondinen treffen sich. Die eine prahlt: "Ich habe gestern einen Rekord geschlagen!" "Wieso?" fragt die andere "Ich habe ein Puzzle nur in 3 Wochen gemacht, obwohl auf der Packung geschrieben steht 3-5 Jahre!"

Das erste Skript hängt mit dem richtigen Verstehen der Aufschrift „3-5 Jahre" als Alter von 3-5 Jahren, wobei es im zweiten Skript um die Interpretation als Zeitdauer geht. Mit dem ersten Skript sind folgende Elemente verbunden, d.h. Alter (3-5 Jahre), richtiges Verstehen, das Merkmal [+normal ] bzw. [+nicht dumm]. Die Elemente des zweiten Skripts, d.h. Zeitdauer (3-5 Jahre), falsches Verstehen und das Merkmal [+dumm] stehen in einer Opposition zum Skript 1.

Raskin führt im Rahmen der Skripttheorie den Begriff der Ambiguität ${ }^{19}$ ein. Darunter wird der Doppelsinn mit seiner besonderen Funktion in einem Witztext verstanden. Die Doppeldeutigkeit lässt nicht nur eine, sondern auch zahlreiche verschiedene Interpretationen eines humoristischen Inhalts zu. Der Rezipient greift gewöhnlich in erster Linie zu der ersten bekannten Bedeutungsvariante. Erst das weitere Lesen oder Hören des Textes macht ihn darauf aufmerksam, dass eine zweite Bedeutungsvariante mit im Spiel sein kann und sogar auch ist. Raskin nennt folgende Typen von Ambiguität:

- reguläre Ambiguität, worunter die Polysemie und Homonymie verstanden werden

(14) Ein Polizist zu einem in Deutschland lebenden Türken: „Könnten Sie sich bitte ausweisen?" Der Türke antwortet: "Wieso sollte ich, ich lebe gerne in Deutschland!"20

\footnotetext{
${ }^{19}$ Neben der Ambiguität wird von Raskin auch der Begriff Widerspruch genannt.

${ }^{20}$ Dieser Witz erscheint in einer Folge der Sendung „Was guckst du?”
} 
In diesem Fall wird das Verb sich ausweisen mit dem jdn ausweisen verwechselt.

- figurative Ambiguität

(15) Der Chef brüllt seinen neuen Mitarbeiter an: "Bringen Sie etwas zustande, was Hand und Fuß hat?" Der Buchhalter seufzt nur: "Nicht, solange Ihre Tochter die Pille nimmt!"

Es liegt hier der Wechsel von der übertragenen Bedeutung von Hand und Fu $\beta$ haben als 'gut durchdacht sein' zur wortwörtlichen von Hand und Fu $\beta$ als Bezeichnungen der menschlichen Körperteile vor. Im Skript 2. wird das zusätzliche Element Sex eingeführt.

- syntaktische Ambiguität

(16) Eine Frau fragt den Verkäufer: "Kann ich die Bluse im Schaufenster anprobieren?" Der Verkäufer erwidert überrascht: "Sehr gern, tun Sie das, aber Sie können es auch in der Kabine tun"

Geschickt wird in diesem Fall die Wortgruppe im Schaufenster gebraucht, die einerseits als Attribut zu Bluse und andererseits als Lokalergänzung zu anprobieren verstanden werden kann, woraus das Witzige resultiert.

- situative Ambiguität

(17) "Ist der Doktor zu Hause?" flüstert der Patient mit der Erkältungsstimme.

"Nein", haucht die hübsche Frau des Arztes, "kommen Sie doch schnell herein!"

Das Doktorskript und das Verführungsskript stehen in der Opposition Sex vs. kein Sex zueinander. Der situative Kontext ist doppeldeutig. Die Konstruktion des Witzes lässt den Leser bzw. Hörer die Pointe nicht voraussehen.

- quasi Ambiguität auf Grund phonetischer Mehrdeutigkeit

Diese Erscheinung wurde bereits im Witz (12) besprochen, 


\section{(12) Ein Chinese kommt mit zwei Blondinen aus der Bäckerei. Warum? Er wollte zwei Blödchen!}

wo Brötchen und Blödchen als Pseudohomophone gebraucht werden.

Die Allgemeine Theorie des Verbalen Humors (General Theory of Verbal Humour) von Attardo/Raskin [1991] basiert auf der semantischen Skripttheorie und wird als Erweiterung der früher genannten Semantischen Skript Theorie verstanden. Neben der semantischen Ebene werden noch weitere Faktoren genannt:

- logischer Mechanismus, d.h. logische Regeln bei der Umschaltung vom Skript zum Skript

- Situation, also die im Witz präsentierte Szene

- Zielscheibe, d.h. eine Person oder eine Sache mit ihren charakteristischen Eigenschaften,

- narrative Strategie, also die Gattung des Witzes

- Form und Struktur des Witzes, d.h. Erzählung, Dialog oder aber FrageAntwort-Konstruktion

Außerdem wird noch dem Vorhandensein des für die richtige Interpretation erwarteten Wissens eine große Bedeutung zugeschrieben.

Es fällt bei der Analyse des Humoristischen auf, dass es zur Vermischung verschiedener Formen des Komischen kommt, demzufolge kann die scherzhafte Kommunikation als ein gelungenes Zusammenspiel von Komik, Humor und Witzigkeit verstanden werden.

In der Scherzkommunikation tritt noch klarer als in anderen Kommunikationsformen zutage, dass wir es mit einem kreativen und ganzheitlichen Prozeß zu tun haben, der heuristisch arbeitet und mit einem gewissen Grad an Vagtheit gut zurechtkommt [Kotthoff 1998a, S. 2]

Bei der Erzeugung der Struktur eines witzigen gesprochenen bzw. geschriebenen Textes muss an die Pointe gedacht werden, die als ein überraschendes Ende definiert wird. Meistens hat sie eine solche Struktur, dass sie mit der Doppeldeutigkeit und der damit verbundenen Bisoziation zusammenhängt. Dieses Unerwartete ist hier am wichtigsten, es soll für einen der beiden Gesprächspartner vor allem nicht vorauszusehen sein. Das hat zur Folge, dass ein witziger Text nicht nur eine, sondern eine ganze Vielfalt von Interpretationen zulässt. In der Pointe wird die Inkongruenz der scheinbar unpassenden Elemente aufgelöst. Beim Hören oder Lesen 
der witzigen Texte werden von den Gesprächspartnern weitere Kontexte mitproduziert, was auf die genannte Bisoziation der scheinbar inkongruenten Elemente zurückzuführen ist. Der einen humoristischen Text schaffende Gesprächspartner verzichtet auf den gewöhnlichen Code, verlässt seine Rahmen und produziert ungeahnte Zusammenhänge. Auf diese Weise kommt es zur Entstehung eines als witzig einzustufenden Textes. Die Scherzkommunikation ist somit als ein kreativer und ganzheitlicher Prozess zu verstehen. 


\section{WITZ ALS GRUNDEINHEIT DER SCHERZHAFTEN KOMMUNIKATION}

\subsection{Begriff des Witzes}

Der Gegenstand der Humorforschung ist neben dem Humor, der als psychosozialo-linguistische Erscheinung aufzufassen ist, auch die Form des Humoristischen - der Witz. Diese Ausdrucksform wird vor allem in Bezug auf ihre Form und Funktion besprochen, d.h. es ist meist eine kurze Unterhaltungsform, mit einer bestimmten Struktur - Thema, Zielscheibe, Motiv und dem dazu gehörenden unerwarteten Ende - Pointe. Die lustigen Inhalte werden aber von dem Autor absichtlich versteckt, so dass man meist zwischen den Zeilen lesen muss, um den Sinn der Aussage zu verstehen. Der Witz soll so konzipiert sein, dass er seine Rezipienten zum Lachen bringt und ihnen somit die Entspannung garantiert. Oft werden die Witze als eine Art Flucht vor dem Alltag verstanden, indem sie sich auf die ganz normalen Alltagssituationen beziehen, sie im Zerrspiegel darstellen, so dass trotz aller damit zusammenhängenden, oft nicht nur positiven Assoziationen gelacht werden kann, genauso wie die Maxime sagt „Humor ist, wenn man trotzdem lacht.“

Im Rahmen der Humorforschung wird vor allem auf den Charakter der witzigen Texte hingewiesen. Es wird die Frage beantwortet, was das Komische ausmacht, welche Elemente dabei eine wichtige Rolle spielen. Den Ausgangspunkt für alle Erwägungen in diesem Bereich bilden die bereits erwähnten Forschungen von Freud [1905], der u.a. den Witz mit der Traumdeutung gleichsetzt. So wie im Traum kommen auch im Witz alle versteckten Träume und Triebe des Menschen zum Vorschein. Die Träume werden passiv und die Witze aktiv erlebt.

Des Weiteren sollen noch die Definitionen von Plessner [1950] und Röhrich [1977] genannt werden. Plessner versteht den Witz als eine besondere Form des Komischen, was zum Lachen provoziert und den Rezipienten unterhalten soll. Bei Röhrich wird der Witz als die wichtigste Gattung der Volkserzählung verstanden. Es wird vor allem die Art und Weise der Entstehung und Weiterleitung der humoristischen Texte besprochen. Nach Röhrich ist der Witz eine kurze Erzählung, die 
ein überraschendes Ende hat, das als Pointe bezeichnet wird, die den Hörer bzw. Leser zum Lachen bringen soll.

In der Soziologie wird der Witz als diese Form der menschlichen Kommunikation verstanden, die nur in einem bestimmten sozialen Kontext sinnvoll und verständlich wirkt.

\subsection{Sachwitz und Sprachwitz}

Es wird auch zwischen dem Sprachwitz und dem Sachwitz unterschieden. Man geht in erster Linie davon aus, was im Vordergrund steht - die Sprache als Mittel des Humoristischen oder vielleicht alles Außersprachliche, das den situativen Kontext ausmacht. Dabei muss aber betont werden, dass im Rahmen der sog. Medienkomik die beiden genutzt werden - das Sprachliche wird mit dem Außersprachlichen so kombiniert, dass sich daraus das Komische ergibt. Damit hängt auch die Unterscheidung zwischen den verschiedenen Möglichkeiten der Pointeerzeugung zusammen.

Im Falle eines Sachwitzes haben wir es mit einer solchen Art Witzigkeit zu tun, dass komische Situationen in den Vordergrund treten, indem zahlreiche Figuren gegen alle möglichen Tabus kämpfen, nicht selten mit ihrer Verhaltensweise gegen Regeln und Normen verstoßen, gar nicht erwartete Reaktionen zeigen und somit den Rezipienten auch zum Lachen bringen. Dem situativen Kontext kann zuerst ein breites Spektrum an sprachlichen Mitteln angepasst werden, was dann geschickt gebraucht werden kann, wenn ein Sketchtext entstehen soll. Des Weiteren bekommt auch der Zuschauer die Möglichkeit, die Pointe auf seine eigene Art und Weise zu entschlüsseln und somit den ganzen Witz zu verstehen. Interessant ist dabei die Tatsache, dass die Sachwitze international verständlich sein können, was aber in erster Linie aus dem Vorwissen der Rezipienten resultiert, das bei der Rezeption dieser Art Witze verlangt wird. Es geht dabei um das politische, soziale, kulturelle bzw. konfessionelle Wissen.

Im Vergleich zu den Sachwitzen, die aus dem situativen Kontext resultieren, hängen die Sprachwitze, anders Wortwitze genannt, mit allen Elementen des jeweiligen Sprachsystems zusammen, die als Mittel des Humoristischen eingesetzt werden.

Im Rahmen der sprachlichen Kommunikation entscheidet jeder der Gesprächspartner darüber, zu welchen Mitteln er greift, um das Beabsichtigte möglichst schnell, präzise und gleichzeitig mit wenig Mitteln zum Ausdruck zu bringen, was vor allem in den kompakten Aussagen zu sehen ist. Einerseits gilt die 
Sprache als Medium der Verständigung, der Mitteilung und des Informierens, andererseits aber dient sie dem Ausdruck der Emotionen und Gefühle oder sogar wird sie auch als Mittel der Unterhaltung verstanden, unabhängig davon, ob es schriftlicher oder mündlicher Humor ist. Das Inventar von Vokabeln, das jedem Gesprächspartner individuell „zugeschrieben“ steht, führt dazu, dass die geführten Gespräche verschieden konzipiert und somit auch unterschiedlich wirkend sein können, manchmal sogar gelingt die Kommunikation gar nicht. Die dabei aufkommenden Missverständnisse gehen oft auf die fehlende Kenntnis des Wortschatzes oder sogar die der Sprache zurück, die für einen der beiden Gesprächspartner fremd ist. Diese Mechanismen werden absichtlich für die Zwecke der Unterhaltung gebraucht, indem man die Texte schreibt, die sich auf die bereits genannten Erscheinungen berufen. Natürlich darf man nicht außer Acht lassen, wie stark die beabsichtigte Wirkung der humoristischen Texte sein soll, so dass im Endeffekt das Ziel erreicht werden kann.

Um das Komische zu erzielen, greifen die Sprecher oft zu den Mitteln, die ihnen das Spiel mit der Sprache und somit auch mit dem Gesprächspartner ermöglichen. Dazu gehören vor allem Wortspiele, Doppeldeutigkeiten, Kontaminationen und Paronomasie [Ostromęcka-Frączak 2008]. Nicht weniger wichtig sind die Merkmale der gewählten Lexik auf der semantischen Ebene, das heißt vor allem ihre Polysemie und auf der morphologisch-semantischen - die Homonymie. Alle diese Merkmale haben an sich, dass sie für die Unterhaltungszwecke gebraucht werden können, weil sie den Wert des gebildeten witzigen Textes erhöhen.

Es muss darauf hingewiesen werden, dass für die Scherzkommunikation ${ }^{21}$ die Mitproduktion weiterer Kontexte charakteristisch ist. Der Zuschauer bzw. der Hörer will den Witztext richtig verstehen. Verschiedene Assoziationen und die Herstellung der nicht vorauszusehenden Zusammenhänge sind Zeichen für die Scherzkommunikation, die als ein ganzer und kreativer Prozess zu verstehen ist. Von den Rezipienten wird verlangt, dass sie ein besonderes Sprachgefühl haben, was ihnen demzufolge ermöglicht, die in den Witzen versteckten Informationen zuerst zu finden und sie dann zu entschlüsseln. Dabei wird noch von ihnen erwartet, dass sie eine gewisse Reaktion zeigen, d.h. das Gehörte akzeptieren, es richtig interpretieren und zum Schluss noch darüber lachen. In diesem Falle wird von den Rezipienten also nicht nur das allgemeine Vorwissen verlangt, sondern auch noch das Wissen über die jeweilige Sprache und deren Elemente, ihre Bedeutungsvarianten usw.

${ }^{21}$ Die Scherzkommunikation wird als Gegenteil der sog. ernsthaften Kommunikation verstanden. Die erste hängt mit der Mitproduktion der Kontexte - sowohl der sprachlichen als auch der situativen - zusammen, was die Folge der ständigen Kopplung von Kognition und Kommunikation ist. Mehr dazu: Kotthoff [1998b]. 


\subsection{Sketch und der konversationelle Humor}

In einem Sketch ${ }^{22}$ hat man es in erster Linie mit dem konversationellen Humor zu tun. Es ist ein Sprachwitz, in dem das breite Spektrum der Sprache auszunutzen ist, indem man vor allem auf die Polysemie, Homonymie und Homophonie $^{23}$ der jeweiligen, in unserem Falle der deutschen und der polnischen, Lexik zurückgreift. Es stellt sich heraus, dass komische Effekte auch beim Übertreiben im Gebrauch falscher oder gebrochener Flexionsformen oder bei der Aussprache der fremdsprachigen Elemente entstehen können.

Selbst die Unterhaltungskünstler, wie Kaya Yanar ${ }^{24}$, behaupten, dass im Zentrum ihres Interesses die Sprachen als Mittel der Unterhaltung stünden, denn

Sprachen haben Charaktereigenschaften [...] Eine Sprache kann ja auch ein Lebensgefühl rüberbringen.

Der konversationelle Humor, der für die Sketchtexte typisch ist, kann folgendermaßen charakterisiert werden [Kotthoff 1998b]:

- Am Anfang und zum Schluss darf gelacht werden.

- Das Spiel mit der Sprache betrifft auch die sog. institutionaliserten Inhalte.

- Das Humoristische besteht aus der Bisoziation, der Ambiguität und den Interpretationen.

- Beim Entstehen des Komischen wird gemeinsames Wissen vorausgesetzt.

- Die Kreativität und die Phantasie spielen im Rahmen des Humoristischen eine besondere Rolle.

- Die nicht-ernste Kommunikation ermöglicht das Anspielen auf die Gefühle und das soziale Umfeld mit ihren Eigenschaften.

- Die Wirkung des Humors hängt mit der guten Stimmung zusammen.

${ }^{22}$ Sketch, der; -[es], -e[s] u. -s, (auch:) Sketsch, der; -[es], -e [engl. sketch = Skizze, Stegreifstudie $<$ niederl. schets $=$ Entwurf $<$ ital. schizzo, Skizze]: (bes. im Kabarett od. Varieté aufgeführte) kurze, effektvolle Szene mit meist witziger Pointierung: einen S. aufführen; die Fernsehshow Spitting Image..., bei der Kautschuk-Karikaturen mit ätzenden Sketches Aktuelles aus Kabinett und Königshaus parodieren [Spiegel 13, 1986, 286]. 2000 Dudenverlag

${ }^{23}$ Mehrdeutigkeit, mehr|deu|tig <Adj. >: 1. aufgrund mehrerer Bedeutungen missverständlich. 2. (bes. Fachspr.) mehrere Deutungen zulassend.; Ho|mo|ny|mie, die; - [griech. homonymía = Gleichnamigkeit] (Sprachw.): lautliche Übereinstimmung von Wörtern mit verschiedener Bedeutung [u. Herkunft]; Ho|mo|pho|nie, die; - [griech. homophonía = Gleichklang] (Musik): Satztechnik, bei der die Melodiestimme hervortritt $u$. alle anderen Stimmen begleitend zurücktreten. 2000 Dudenverlag

${ }^{24}$ Kaya Yanar, geb.1973, deutscher Stand-up-Comedian, bekannt geworden dank der TVSendung Was guckst du? ; Vgl. dazu Wittner J., Interview Kaya Yanar, von stern.de/ unterhaltung $-5.3 .2008$ 
Wenn von dem konversationellen Humor die Rede ist, sollen an dieser Stelle auch seine Strategien genannt werden, d.h. Anspielung, Stimmungszeichen, Wortspiel und Platzierung.

Interessant ist auch die Antwort auf die Frage nach dem Thema, der Zielscheibe und dem Motiv, die als Bestandteile der humoristischen Texte zu verstehen sind. Bei der semantischen Analyse soll vor allem auf die Pointe geachtet werden, denn die Witze haben eine bestimmte Funktion, nämlich die der Sinnübertragung an die Zuhörer bzw. Leser. Darauf weist vor allem Raskin (1985) hin. Er reduziert die Humortheorie auf die verbale Witzigkeit und nennt den Begriff der Skripts als die Information, die um ein Wort herum existiert. Der verbale Humor hängt demzufolge mit dem Einsatz einzelner Subsysteme der Sprache zum Zwecke des Komischen zusammen. Die Texte der Witze können als Hybriden bezeichnet werden, weil sie an sich eine Mischung aus verschiedenen Typen der witzigen Texte sind.

\subsection{Zum Ethnowitz}

Das vorhandene Vorwissen aus möglichst vielen Bereichen und die Kenntnis der immer noch geltenden Stereotype verschiedener Art hängen mit der Rezeption und der richtigen, d.h. der erwarteten Interpretation der ethnischen Witze zusammen. Die Texte aus dieser Gruppe von Witzen werden noch im Falle eines lustigen Bühnenstücks - des Sketches - mit allem Nonverbalen unterstützt, was vor allem an bestimmte stereotype Vorstellung einer konkreten Nation angehängt wird [Sikorska-Bujnowicz 2012].

Es sind Witze über und von Nationen und haben einen internationalen Charakter. Bei der Klassifizierung der Texte dieser Art spielen vor allem die Stereotype eine Rolle, z.B.

- Man kritisiert an den Schotten ihren Geiz.

(18) Die schottische Nationalmannschaft hat ein Spiel gewonnen. Der Trainer kommt in die Kabine und sagt: "Jungs, ihr wart großartig.

Ihr habt euch eine Erfrischung redlich verdient. Los, John, mach das Fenster auf! "

(19) In der Zeitung: Schottisches Taxi stürzt in einen Fluss: 42 Tote... 
(20) Woran erkennt man ein schottisches Schiff? Keine Möwen!...

- Italiener werden als faule und laute Machos präsentiert.

(21) Wieso sind die Italiener so klein?

Wenn sie groß sind, müssen sie arbeiten gehen.

(22) Ein Amerikaner und ein Italiener treffen sich in einer Kneipe. Sagt der Amerikaner: "Gib mir einen Hammer und ich bau dir einen Flugzeugträger!"

Sagt der Italiener: "Gib mir deine Tochter und ich mach dir die Besatzung dazu!"

(23) Eine italienische Reisegruppe besichtigt die berühmten Niagarafälle. Der Fremdenführer macht sie aufmerksam:

"Meine Damen und Herren, wenn Sie für einen Moment Ihre Unterhaltung einstellen würden, könnten Sie das gewaltige Tosen und Brausen des Wasserfalls hören."

- Türken werden als faule, gewalttätige und klauende Nation ausgelacht

(24) Kommt ein Türke zum Arbeitsamt.

Berater: ... und was kann ich für Sie tun?

Ali: Ich brauche einen Job.

Berater: Mann haben Sie Glück, ich hab sogar schon einen Job für Sie im Angebot.

Ali: Im ernst?

Berater: Ja, Sie werden eine große Luxuslimousine fahren, im Büro sitzen und 600.000 DM im Jahr verdienen.

Ali: Sagen Sie mal, wollen sie mich verarschen?

Berater: Na hören Sie mal, wer hat den damit angefangen?

(25) Ein Türke ist auf der Autobahn unterwegs. Plötzlich bekommt er einen Plattfuss. Als er gerade dabei war das Rad zu wechseln, kommt ein Pole angefahren, steigt aus und geht zu dem Türken und reist das Radio raus. Darauf der Türke wütend: "Ey, spinnst du! "Da meinte der Pole: "Pssst, ich Radio und du Reifen."

(26) Welches ist das freundlichste Volk auf der Welt?

Die Türken, sie kommen mit 20 Mann auf dich zu und fragen dich, ob du ein Problem hast. 
- Auch Polen erscheinen als Zielscheibe der Ethnowitze, wobei schon im genannten Witz (25) auf das Stereotyp gezeigt wird, dass jeder Pole stiehlt und dazu noch viel zu viel Alkohol trinkt

(27) Ein Satz mit 10 Wörtern und 4 Lügen?

Ein ehrlicher Pole fährt nüchtern mit seinem Wagen

(28) Was ist in Polen der Unterschied zwischen einer Hochzeit und einer Beerdigung? Auf letzterer ist einer weniger besoffen.

(29) In Polen hat man nie Probleme, wenn man im Halteverbot parkt: Bis die Politesse da ist, ist der Wagen eh geklaut. ${ }^{25}$

Im Rahmen der Humorforschung nehmen die Ethnowitze einen besonderen Platz ein, was darauf zurückzuführen ist, dass sie zeit- und raumunabhängig sind, weil sie doch überall verstanden und gleich interpretiert werden [Sikorska-Bujnowicz 2011a]. Als Witze dieser Art werden Texte einerseits über Nationen und andererseits von Nationen verstanden, wobei die ersteren überwiegen. Dabei können die Unterscheidungsfaktoren genannt werden, wie

- nationale Zugehörigkeit

- Religion

- ethnische Zugehörigkeit.

Alle genannten Faktoren sind bei der Bildung der Ethnowitze von Bedeutung, weil diese meist die Mentalität der jeweiligen Nation im Zerrspiegel darstellen. Man richtet sich in erster Linie nach den Stereotypen, die überzeitlich sind und überall gelten.

Humor lässt sich nicht als sprachliches Phänomen isolieren mit dem Hinweis, ersei mit einem Signifikat namens ,nationaler Kulturraum“ identisch - umso weniger, als viele Witze und Anekdoten mühelos Sprach- und Landesgrenzen überwinden und von den Rezipienten nach einer Weile behandelt werden wie ihr eigenes kulturelles Besitztum [Frahm 2008, S. 24].

Die ethnischen Stereotype betreffen alle möglichen Eigenschaften, die einer bestimmten nationalen Gruppe mental zugeschrieben werden [Rösch O. (Hrsg.) 2000]. Ihre besondere Wirkung kann vor allem deswegen festgestellt werden, weil die Witze dieser Art die ganze Gruppe, nicht nur ein Individuum betreffen und

${ }^{25}$ Die im Text zitierten Ethowitze entstammen den Internetseiten: http://www.grocceni.com/, http://www.lustich.de/. 
somit eine generalisierende Funktion haben. Diese Verallgemeinerung hat eher einen destruktiven Charakter, weil mit den verallgemeinerten Informationen Menschen aus einer anderen Kultur eher negativ bewertet werden. Es muss jedoch auch darauf hingewiesen werden, dass ethnische Stereotype neben allem, was mit der Kritik und dem scharfen Beurteilen einer anderen sozialen, beruflichen oder ethnischen Gruppe verbunden ist, auch konstruktiv wirken, weil mit ihrer Hilfe eine gewisse Zusammenstellung von Informationen über Gruppen ermöglicht wird und unabhängig davon, welche soziale Gruppe gemeint wird, bestimmte Signale und Muster strukturiert und angeordnet werden.

Quasthoff [1989] nennt folgende Funktionen von Stereotypen

- kognitive Funktion

- affektive Funktion

- soziale Funktion

Die kognitive Funktion der Stereotype besteht darin, dass die Informationen über eine fremde Kultur dank der Generalisierung verarbeitet werden können, so dass sie im Endeffekt eben mit der jeweiligen Kultur assoziiert werden, was auf einen dabei entstehenden interkulturellen Lernmechanismus zurückzuführen ist.

Im Falle der affektiven Funktion wird davon ausgegangen, dass psychische Einstellung des Individuums bzw. einer ganzen Gruppe zu einer anderen, die eine fremde ethnische Minderheit bildet, rationalisiert wird. Der interkulturelle Kontakt trägt dazu bei, dass verschiedene Nationen miteinander in Berührung kommen und sich somit im Laufe der Zeit ihrer eigenen ethnischen Identität immer bewusster werden und die der anderen Nation auch anerkennen.

Aus Angst vor dem Fremden und Unbekannten entstehen jedoch in den meisten Fällen die Vorurteile. Darauf habe ich schon in einem meiner Artikel hingewiesen [Sikorska-Bujnowicz 2012b, S. 19]:

Das fehlende kulturelle Wissen trägt dazu bei, dass im Witz nur teilweise wahre Informationen vermittelt werden. Es entstehen demzufolge die Texte, die vor allem auf dem Vom-Hören-SagenBekannten basieren und nicht auf der Wahrheit. An dieser Stelle muss noch darauf hingewiesen werden, dass alles, was mit den Stereotypen zusammenhängt, in enger Verbindung mit den ethnischen Vorurteilen steht. Infolge dessen bekommen die witzigen Texte eine generalisierende Funktion. Einerseits dienen sie der Kritik oder der scharfen Auseinandersetzung mit der jeweiligen Nation, andererseits aber werden sie zum Mittel der Integration. Es wird betont, dass die ethnischen Stereotype sowohl als Denkstereotype als auch als sprachliche Stereotype zu verstehen sind.

Die soziale Funktion ist vor allem in der Organisation und Strukturierung der Gruppen und in der Herausbildung der alltäglichen Relationen zwischen einer 
bestimmten ethnischen Gruppe und dem Rest der Gesellschaft zu sehen. Es geht dabei nicht nur um das Sich-Abheben vom Rest, sondern eher um das Zusammenleben innerhalb einer und derselben Gesellschaft. Die Ethnowitze als überzeitlich geltende und raumunabhängige Einheiten der scherzhaften Kommunikation werden sogar als internationales Gut verstanden. 



\section{HUMOR IN DEN MEDIEN}

Für den Humor in den Medien ist es charakteristisch, dass mit vielen verschiedenen Formaten und demzufolge auch verschiedenen Ebenen gleichzeitig gearbeitet wird, denn das Publikum soll auf diese Weise mit vielen möglichen Mitteln des Humoristischen konfrontiert werden und demzufolge soll mindestens eine Ebene verstanden werden - entweder die außersprachliche oder die der Sprache. Neben den notwendigen Sprachkenntnissen wird von den Rezipienten auch das Verstehen der weiteren Ausdrucksebenen verlangt, denn nur so können die beiden Bereiche - der sprachliche und der außersprachliche zusammen richtig verstanden, entschlüsselt und interpretiert werden. Die für bestimmte witzige Figuren charakteristischen Eigenschaften, wie das Äußere (Kleidung, Frisur, Maske), die Körperhaltung und - bewegung, ihre typische Körpersprache (Mimik, Gestik), aber auch Stimme, Akzent oder Intonation werden dem Zuschauer gleichzeitig mit einem witzigen Text angeboten, damit dieser die weiteren Kontexte mitproduzieren kann und das Versteckte entschlüsselt, was ihn im Endeffekt zum Lachen bringen soll. Der Rezipient hat noch eine weitere Aufgabe - er soll sich geschickt zwischen verschiedenen Medieninhalten bewegen, die im Rahmen der jeweiligen Comedysendungen angeboten werden, was wiederum auf den intertextuellen Charakter der im Fernsehen angebotenen Witze hinweist.

\subsection{Fernsehsendung als Element der Massenkommunikation}

Im Rahmen der deutschen Humorforschung unterscheidet Kotthoff [2004a, S. 1] zwischen der Alltagskomik und der Medienkomik und weist darauf hin, dass zwischen ihnen dann doch Differenzen zu nennen sind, unter anderem die Tatsache, dass

[...] im Alltagshumor auch auf Wissen angespielt wird, welches nur die beteiligten haben. Dazu liegt die Komik oft nur auf einer oder zwei Ebenen (zum Beispiel Worten und Mimik), während sie im Film zum Beispiel auf mehreren Ebenen angesiedelt wird. 
Dabei darf aber nicht vergessen werden, wie die beiden Arten Komik einander beeinflussen, was natürlich nicht zu bedeuten hat, dass der Rezipient genau auf dieselbe Art und Weise auf einen witzigen, in den Medien angebotenen Text vs. auf eine witzige Situation aus dem Alltag reagiert. In jedem Fall wird darauf geachtet, dass das im Fernsehen angebotene Witzige uns als Rezipienten nicht persönlich verletzt, wobei ein und derselbe Witz auf unsere Kosten doch anders wahrgenommen und interpretiert wird. Nicht weniger wichtig ist dabei, inwieweit man in einem Witz gegen alle Normen und Gesetze verstoßen darf, ohne dass man die Gefühle anderer Gesprächspartner verletzt. Damit meine ich auch die Grenzen des guten Geschmacks, die doch leicht und schnell überschritten werden können, ohne dass sich die Autoren irgendwelche Gedanken darüber machen, dass sie mit ihren Texten die Gesetze verschiedener Art verletzen. Beim Schreiben der witzigen Texte geht man gewöhnlich in erster Linie davon aus, dass am Ende über das Erzählte gelacht werden soll, demzufolge greift man zu verschiedenen Mitteln, um dieses Ziel zu erreichen. Zur Zielscheibe werden dann die Ausländer, die einer für sie neuen und bisher unbekannten Sprache nicht mächtig sind und immer wieder wegen der fehlenden Sprachkenntnisse verschiedene Fehler begehen (alle Figuren in „Was guckst du?“, bei „Paranienormalni“ - Chinesen, die während einer Hochzeit auftreten sollen - Sketch „Wesele w chińskiej restauracji“, bei „Ani Mru Mru“ - ein Chinese, der in seinem Restaurant die Bestellung aufnimmt - Sketch „Chińska restauracja“usw.)

Auffällig ist, wie sehr man sich in Scherzen auf geteiltes Wissen verlässt. Insofern ist es sehr geeignet, Gruppenzugehörigkeit zu bekräftigen, die ingroup von der out-group abzugrenzen, was in der Geschichte der Humorforschung von Henri Bergson über Sigmund Freud bis zu Helmuth Plessner auch immer wieder betont wurde. Als weitere wichtige Funktion wäre zu nennen, dass man sich im Scherz implizit einer geteilten Moral versichert. Explizites Moralisieren hat in unserer Gesellschaft einen schlechten Ruf.[Ebenda, S. 5]

Im Rahmen der Kommunikation wird nicht nur zwischen der ernsten und der scherzhaften, sondern auch zwischen dem primären und dem sekundären Kommunikationsprozess ${ }^{26}$ unterschieden. Wenn mit dem Begriff der primären Kommunikation die face-to-face Kommunikation gemeint wird, geht es im Falle der Sekundärkommunikation um einen indirekten Prozess.

Die Aussagen werden in der letzteren mit Hilfe technischer Geräte vermittelt, wobei es sowohl eine räumliche als auch eine zeitliche Distanz gibt. Die Gesprächspartner sind voneinander getrennt, die Informationen werden an das

${ }^{26}$ Vgl. mehr dazu Gabler Wirtschaftslexikon, Stichwort: Massenkommunikation, http://wirtschaftslexikon.gabler.de/Archiv/55023/massenkommunikation-v5.html (Stand vom 2012). 
Publikum weitergeleitet. Die Frequenz und die Intensität dieser Art Kommunikationssituationen sind eher geringer als die der primären Kommunikation. Darunter wird die so genannte Massenkommunikation verstanden. Dabei soll beachtet werden, dass auf diese Weise nicht nur einzelne Kommunikationspartner angesprochen werden, sondern ein ganzes Publikum. Die Informationskanäle sind nur Ein-Weg-Kanäle, vor allem wegen der für die Gesprächspartner nicht bestehenden Möglichkeit, an diesem Prozess des Gedankenaustausches aktiv, im Sinne der face-to-face Kommunikation, teilzunehmen [Maletzke 1963]. Mit der Massenkommunikation hängt ein disperses Publikum zusammen, das der Struktur nach heterogen ist.

Zu den Medien, die diese Art Kommunikation ermöglichen, gehört das Fernsehen. Über dieses Medium werden nicht nur Nachrichten aus der ganzen Welt vermittelt, sondern es wird auch darauf geachtet, die Zuschauer zu unterhalten. Trotz der zwischen dem Textproduzenten und den Rezipienten bestehenden Distanz, die nur selten aufgehoben werden kann, nur dann, wenn z.B. im Fernsehstudio eine Live-Show produziert wird, zu der auch das Publikum eingeladen wird, kann auch von dem Feedback die Rede sein, denn jeder Sender hat seine Homepage, wo er auch den Zuschauern zugänglich ist und mit ihnen im Kontakt bleibt. So ist es auch im Falle der Sendung „Was guckst du?“, auf deren Seite alle für die Zuschauer wichtigen Informationen zu lesen sind - Termine der nächsten Ausstrahlung, Konzerttermine, Ereignisse aus dem Leben der Darsteller usw. Auch die polnischen Kabarettgruppen bleiben mit ihren Zuhörern bzw. Zuschauern dank ihrer Internetseiten - http://www.paranienormalni.pl/, http://www. animrumru.pl/ und http://www.kabaretmoralnegoniepokoju.pl/ im Kontakt. Auf diese Weise ist ein weiteres Medium an der Massenkommunikation beteiligt - das Internet. Die Definition der Massenkommunikation, die auf die siebziger Jahre zurückgeht, wird zur Zeit noch um weitere Medien ergänzt, unter denen auch das Internet genannt wird. Es ist dann von Multimedien die Rede, die digital und somit auch interaktiv sind. Im Rahmen der Kommunikation solcher Art hat jeder Kommunikationspartner das gleiche Recht, von dem Medium unabhängig zu sein, in dem Sinne, dass von ihm die zu vermittelnden Botschaften formuliert und zu dem von ihm selbst bestimmten Zeitpunkt weitergeleitet werden können. Bilder, Texte, verschiedene graphische Präsentationen und die dabei in die Sendung eingesetzten Geräusche stehen in verschiedenen Relationen zueinander, d.h. entweder ergänzen sie sich, widersprechen einander oder können gar nicht zusammenpassen. Dem Rezipienten selbst wird dann überlassen, diese Schwierigkeiten zu überwinden. Das Internet als ein neues Medium gibt dem Menschen die Möglichkeit, zeitlich und räumlich unbegrenzt eine Diskussion nicht nur mit einem Kommunikationspartner zu führen, sondern mit vielen auf einmal, nur mit einem 
kleinen Unterschied - das alles läuft virtuell. Das Mündliche wird somit zum Schriftlichen, was jedoch voll von umgangssprachlichen Elementen ist und Merkmale bestimmter Soziolekte aufweisen kann, je nachdem, welche Foren angesprochen werden. Unter den Internetusern gibt es auch solche, für die das Internet als Quelle der witzigen Texte zu verstehen ist, aus der unbegrenzt geschöpft werden kann und in der ständig neue Witze erscheinen - mit der Anmerkung der Autoren bzw. der Erzählenden, wie der Text in seiner Wirkung ist, d.h. ob er lustig, wenig lustig oder gar nicht komisch ist. Die Internetuser bilden ein anonymes Publikum und sind gleichzeitig die Moderatoren, von denen es abhängig ist, welche Witze auf einer konkreten Seite erscheinen und wie sie zu beurteilen sind. Genauso ist es mit den Fernsehzuschauern, die ein verstreutes Publikum bilden, weil sie eine unzählige Gruppe bilden, einander nicht kennen und demzufolge anonym sind, aus verschiedenen sozialen und beruflichen Gruppen kommen, unterschiedliche Meinung zu demselben Thema haben können und innerhalb dieses dispersen Publikums jedoch keine Rolle zugeteilt bekommen, weil es keine klare Struktur und Hierarchie hat. ${ }^{27}$ Nach Meinung von Maletzke ist es jedoch wichtig, dass sich der Sender ein gewisses Bild von seinem Publikum zu machen versucht, um den Zuschauern auf diese Weise ein an sie angepasstes Programm anzubieten. Das ist dank dem unmittelbaren Kontakt mit den Zuschauern möglich, vor allem dann, wenn sie ins Studio eingeladen werden und an der Sendung aktiv teilnehmen. Über das Fernsehen werden dann die Aussagen an das breite, disperse Publikum geleitet und der Zuschauer hat die Möglichkeit, von allen möglichen Programmangeboten etwas für sich zu finden.

Das Feldschema der Massenkommunikation von Maletzke wird des Weiteren von Burkhart und Hömberg [1997] erweitert und es entsteht das Modell, das schon die elektronischen Medien mitberücksichtigt. Die Massenkommunikation wird demzufolge als eine elektronisch mediatisierte Gemeinschaftskommunikation verstanden. Die Vernetzung ermöglicht den Kommunikationspartnern weltweit zu kommunizieren, sogar samt Bild und Ton, ohne dass sie sich von zu Hause fortbewegen. In der heutigen Auffassung der Massenkommunikation erscheinen folgende Faktoren:

- Texte und Bilder sind als Zeichen der Kommunikation zu verstehen.

- Als technische Mittel wird allgemein die Elektronik gemeint.

- Medien können analog oder digital und vernetzt sein.

- Elektronische Medien können entweder als Mittel der öffentlichen oder der privaten Kommunikation verstanden werden.

${ }^{27} \mathrm{Vgl}$. Anm. 26. 
- Der Gedankenaustausch erfolgt anonym, kann zur gleichen Zeit stattfinden oder zeitlich versetzt sein.

- Die räumliche Distanz kann mit Hilfe der neuesten Programme wie ,skype“ zum Teil behoben werden, weil sich die Sprechenden dann sehen können. Es entsteht demzufolge ein quasi face-to-face Gespräch.

- Die Kommunikation betrifft entweder den einzelnen Kommunikationspartner, mehrere von ihnen oder sogar eine unzählige Menge von Rezipienten.

Wenn man eine Fernsehsendung zu analysieren anfängt, denkt man in erster Linie daran, wie eben dieses Verhältnis zwischen dem Moderator als Produzenten und dem Zuschauer als Rezipienten wiedergegeben werden soll - ist das etwas Echtes oder aber nur gespielt? Das ins Fernsehstudio eingeladene Publikum nimmt an dem Gezeigten und Erzählten teil, wovon seine Reaktionen in Form der Gelächter zeugen. Ein disperses Publikum als Rezipient innerhalb des Massenkommunikationsprozesses hat demzufolge seine Vertreter im Studio, die das Programm mit ihren Reaktionen - Lächeln und Lachen - um ein wesentliches Element ergänzen - um den Kommunikationspartner, der zwar selten etwas sagt, dafür aber zuhört und mit seiner Körpersprache der beste Gutachter der jeweiligen Sendung ist, nach dem Motto - „vom Gesicht lässt sich alles ablesen“ - von der Abneigung bis zur Liebe. Wenn der Darsteller seine vorzutragenden Sketchtexte mit den dazu passenden Elementen der Körpersprache ergänzt und diese bewusst einsetzt, um gewisse Reaktionen der Rezipienten zu erzielen, dann geht es um die Erfüllung des Wunsches nach der Bereicherung der Präsentation eines witzigen Textes und demzufolge - um das Lachen. Wenn der Komiker hastig auf der Bühne hin und her läuft, drückt er noch stärker als nur mit den Worten selbst seine Nervosität aus, wenn er zufrieden ist, reibt er sich die Hände, wenn er etwas verschweigen will, legt er einen Finger auf seine Lippen, wenn er mit den Fingern trommelt, ist er nervös usw. - solche Signale als Elemente der Gestik sind in allen Kulturen leicht zu entschlüsseln, weil sie dem Menschen angeboren und nicht erlernt sind. Auch die Mimik, also der Gesichtsausdruck spielt hier eine Rolle. Wenn man langes Gesicht macht, ist man nicht zufrieden, lehnt man jemanden oder etwas ab und man zeigt auf diese Weise seine negativen Gefühle. Das Erstaunen kann vom Gesicht dann abgelesen werden, wenn der Sprecher seine Augenbrauen hochzieht, manchmal dabei auch den Mund aufmacht, wobei die hochgehobenen Augenbrauen selbst auch als Zeichen der Skepsis zu verstehen sind. Eine wichtige Rolle wird dem Blickkontakt zugeschrieben, der ein Zeichen des Interesses an dem Gegenüber ist, wobei kein Augenkontakt das Desinteresse symbolisiert.

Mit der sozialen Norm hängt es zusammen, dass negative Emotionen sprachlich eher nicht formuliert werden sollen, wobei sie jedoch nonverbal ganz gut 
vermittelt werden, ohne dass der Sprechende es will. Diese Signale sind an den Kontext gebunden, sowohl den sprachlichen als auch den außersprachlichen. Die kontrollierte Wahl der anzuwendenden Lexik, die Suche nach den Euphemismen, die Versuche, das Negative nicht so extrem schlecht darzustellen, hängen von der Situation ab, in der die Kommunikation stattfindet. In einem Gespräch face-toface kommt es dazu, dass der Gesprächspartner vom Gesicht und besonders von den Augen seines Gegenübers auch alle versteckten und verheimlichten Informationen ablesen kann, aber natürlich nur dann, wenn der Sender seine Körpersprache nicht steuern kann. 


\section{SPRACHLICHE MITTEL DES HUMORISTISCHEN}

$\mathrm{Zu}$ den wichtigsten sprachlichen Mitteln, mit denen das Komische erzeugt werden kann, gehören die Polysemie, die Homophonie und das Wortspiel. Mit dieser Thematik setzte ich mich schon in meinen früheren Beiträgen zum Problem der sprachlichen Mittel des Humoristischen sowohl im Deutschen als auch im Polnischen auseinander [Sikorska 2009a, 2009b; Sikorska-Bujnowicz 2010, 2011, 2012a, 2012b]. In dieser Arbeit geht es einerseits darum, die genannten Erscheinungen kurz zu besprechen und andereseits auch darum, diese in ihrer Funktion im Rahmen der Scherzkommunikation zu charakterisieren. Die Autoren der Sketchtexte greifen zu den genannten sprachlichen Erscheinungen wegen ihres überzeitlichen Charakters, d.h. wegen ihrer Kraft, das Lustige in einem bestimmten Kontext zu schaffen. Die in den meisten Fällen fehlende Kenntnis verschiedener Bereiche gibt dem Zuschauer oder Zuhörer die bzw. keine Möglichkeit, das im witzigen Text Versteckte zuerst zu finden, dann zu analysieren und zum Schluss zu interpretieren, was im Endeffekt mit dem Lachen, also mit der erwarteten Reaktion, endet.

Am häufigsten wird das Wortspiel auf der Basis der Gleichheit oder Ähnlichkeit der Lautung von mindestens zwei Wörtern mit verschiedenen Bedeutungsvarianten realisiert, was zur Folge hat, dass dann die Ambiguität entsteht. Dies erscheint meist in der Form der Homophone, Homographe, Homonyme oder der Paronomasie. Es ist aber jedoch nicht meine Aufgabe, mich mit all diesen Theorien auseinanderzusetzen, sondern sie nur kurz zu skizzieren.

Für die Zwecke dieser Arbeit schließe ich mich der Auffassung an, dass der Humor eine echte Mischung aus vielen Faktoren ist, die bei der Analyse zu beachten sind. Einerseits geht es um die Sprache, deren Nuancen von den Autoren der Texte ausgenutzt werden und andererseits um die Kooperation des Sprachlichen mit dem Außersprachlichen, was mit dem Charakter des Sketches als Bühnenform zusammenhängt. 


\subsection{Wortspiel als Mittel des Humoristischen}

Laut DUDEN (Großes Wörterbuch der deutschen Sprache) ist das Wortspiel $^{28}$ als ein Spiel mit Worten zu verstehen, in dem das Witzige dadurch zustande kommt, dass man dabei zu den doppeldeutigen Wörtern greift oder zu den Lexemen, die gleich oder ähnlich lauten, wie z.B. im Polnischen das Adjektiv winny, das einerseits eine Ableitung von wino 'Wein' ist und demzufolge die Bedeutung von 'mit einer säuerlichen Geschmacksnote, besonders in Bezug auf Äpfel' hat und andererseits von wina 'Schuld', wenn dann winny im Sinne von 'ein schuldiger Mann' steht. Im Deutschen kann als Beispiel ein Wortpaar genannt werden offen gestanden als 'nicht verschlossen sein' und offen gestanden als 'ehrlich gesagt', das auch in einer witzigen Frage-Antwort-Konstruktion erscheinen kann, wenn ein Angeklagter gefragt wird, wie er die Tat begangen hat und ob die Wohnungstür auf war oder nicht und dann antwortet Offen gestanden hat die Wohnung offen gestanden. Demzufolge ist es also eine Figur der Rhetorik, die auf die Polysemie der gebrauchten Wörter zurückgeht, auf der Verdrehung oder Verwechslung beruht oder aber andere Veränderungen an den eingesetzten Lexemen mitberücksichtigt und dem Autor der humoristischen Texte lustig zu sein scheint.

Diese Art Witze ${ }^{29}$ betreffen verschiedene Bereiche, sind u.a. unter der Kategorie der Computer-Witze zu finden, z.B. in denen man u.a. mit der Mehrdeutigkeit des Verbs surfen ${ }^{30}$ spielt, das sich gar nicht auf eine sportliche Aktivität im Sinne von 'Surfing treiben', sondern auf das Suchen von Informationen im Internet bezieht. Zum Betonen der konkreten witzigen Inhalte wird in diesem Text noch ein Determinativkompositum genannt Surfladen, also 'Laden, in dem man alle beim Surfen nötigen Sachen kaufen kann', das unmittelbar mit dem Surfen als

${ }^{28}$ Wort|spiel, das $<_{0 . P 1}$. : Spiel mit Worten, dessen witziger Effekt bes. auf der Doppeldeutigkeit des gebrauchten Wortes od. auf der gleichen bzw. ähnlichen Lautung zweier aufeinander bezogener Wörter verschiedener Bedeutung beruht: ein witziges, albernes W.; Nach einer Umfrage der New York Times meint nur jeder fünfte Amerikaner, dass es sich bei »Whitewatergate «, wie das unvermeidliche W. lautet, um eine Angelegenheit von nationaler Bedeutung handele (Spiegel 12, 1994, 148). 2000 Dudenverlag.

${ }^{29}$ Alle in diesem Kapitel besprochenen Witze entstammen der Internetseite www.witz.de

${ }^{30}$ sur|fen [,:] <sw. V.> [engl. to surf, zu: surf= Brandung, H. u.]: 1. Surfing betreiben $<$ hat/ ist $>$. 2. a) Windsurfing betreiben $<$ hat $/$ ist $>$ : sie lernt s.; b) surfend ( 2 a) irgendwohin gelangen $<$ ist $>$ : über den See s. 3. (EDV Jargon) (im Internet) wahllos od. gezielt nach Informationen suchen, indem durch das Anklicken von Hyperlinks (b) nacheinander verschiedene Seiten (11) aufgerufen werden $<$ hat/ist $>$ : im Internet s.; Service für Generalisten und Spezialisten, die im Netz nicht surfen, sondern arbeiten (Handelsblatt 20.7.99, 46); <subst.: > Im WWW ... Sie können an einer beliebigen Stelle in das Gewebe einsteigen und dank der Verknüpfungen von Dokument zu Dokument wandern das viel zitierte »Surfen im Web« (Schieb, Internet 102). 2000 Dudenverlag. 
Sportart und nicht mit der Computerbranche zusammenhängt. Das Humoristische wird in dem Moment erzeugt, wenn als Pointe die Frage gestellt wird, in der der außersprachliche Kontext genannt wird, d.h. wenn der Rezipient mit der Tatsache konfrontiert wird, dass der Surfladenbesitzer selbst noch keinen Computer hatte.

\section{(30) Surfen}

Thomas geht zur Party. Fragt er die Partygäste: "Was machst du denn nächstes Wochenende?" - "Surfen" - "...und du?" - "Surfen" - "...und du?" "Auch surfen." Nach hunderten gleichen Antworten geht Thomas zufrieden nach Hause und eröffnet gleich am nächsten Tag einen Surfladen. In einem halben Jahr ist er Pleite. Auf der nächsten Party trifft er seine Freunde und wundert sich, dass keiner bei ihm eingekauft hat. Fragt sein Freund: "Na Thomas, hast du dir endlich auch einen Computer gekauft?"

Auf Polysemie als Träger eines Witzes stößt man auch besonders oft in der Kategorie der Blondinen-Witze, z.B. wo in allen Texten die Blondine die Zielscheibe ist und ihre fehlende Intelligenz zum Thema wird, wobei die Motive verschieden sein können, was im Text mit Hilfe der mehrdeutigen Lexik zum Ausdruck gebracht wird. Die Doppeldeutigkeit des Wortes surfen wird auch in dem nächsten Text ausgenutzt, zu betonen sei hier aber, dass in diesem Falle ein Witz vorliegt, der den beiden Kategorien zugeordnet werden kann - den Computerund den Blondinen-Witzen.

\section{(31) Blondine am Computer \\ Warum schüttet eine Blondine Wasser über den Computer? Damit sie auch mal surfen kann!}

Der nächste Text geht auf die unzureichende Kenntnis des Deutschen zurück und zeigt die Frau als Wesen, das alles entweder nur mit Sex oder mit Fußball assoziiert. Im folgenden Witz wird nämlich mit den Bedeutungsvarianten der Verben anmachen $^{31}$ als anfeuern ${ }^{32}$ gespielt, die von der Hauptfigur in der nicht richtigen

${ }^{31}$ an|ma|chen <sw.V.; hat>: 1. (ugs.) befestigen, anbringen: Gardinen a.; ein Schild an der Haustür a. 2. (ugs.) a) anschalten; einschalten: die Lampe, das Radio a.; wir müssen die Heizung a.; mach doch bitte mal das Licht an!; b) anzünden: Feuer a. 3. a) mischend gebrauchsfertig machen, zubereiten, anrühren: Gips, Mörtel a.; Salat mit Öl und Essig a.; Der Teig für Mutters Kuchen wird nicht mit Wasser angemacht (Strittmatter, Der Laden 163); b) panschen (1): Sie verkauften ihm auch ein paar Fässchen angemachten Weines (Keller, Romeo 24). 4. (salopp) a) [herausfordernd] ansprechen u. dabei unmissverständlich [sexuelles] Interesse zeigen: Mädchen in der Disko a.; dür- 
Lesart assoziiert werden, d.h. anmachen als 'jdn ansprechen und dabei unmissverständlich sexuelles Interesse zeigen' und anfeuern in der Bedeutungsvariante ' antreiben, anspornen', wobei der Mann das Lexem im wortwörtlichen Sinne meint, d.h. als 'einschalten'

\section{(32) Ofen}

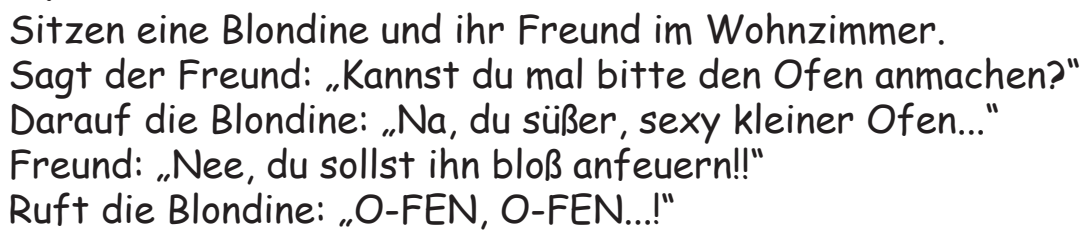

Im nächsten Witzbeispiel wird mit der Bedeutung des auf das polyseme Verb streichen $^{33}$ zurückgehenden Wortes gestrichen als 'mit einer Farbe versehen' im

fen Frauen auch Männer a.?; Obwohl er seine Freundin dabeihatte, machte er mich immer an (Hornschuh, Ich bin 29); so ein paar Typen, ...die haben mich angemacht. Einer wollte mich nach Ibiza einladen (Heim, Traumschiff 164); Und Sieglinde Huntscha versuchte, die Osslieb anzumachen. Die schmusten ganz schön (Grass, Butt 428); b) zum Mitmachen animieren: der Sänger fing an zu klatschen und machte das Publikum an; c) in irgendeiner Weise ansprechen, anregen, reizen; in Stimmung bringen: das macht mich nicht an; Musik, die anmacht (Szene 6, 1983, 50); Sie kommen in den Genuss meines ersten Leserbriefes, weil mich Ihr Artikel über Jogging echt angemacht hat (Spiegel 50, 1978,7); das Einzige, was diesen Mann anmacht, scheint Geld zu sein (Lindlau, Mob 178); mach mich nicht an! (lass mich in Ruhe!); d) jmdm. hartnäckig zusetzen; jmdn. behelligen, belästigen: einen Menschen wegen seines fremdländischen Aussehens a.; Mein Vater macht meine Mutter ständig an, sie gäbe zu viel Geld aus (Christiane, Zoo 20); ...wurden Schüler, die das Etikett im Unterricht trugen, von ihren Lehrern angemacht (Spiegel8, 1982, 28); Ewig fühlt er sich falsch verstanden, falsch zitiert, zu Unrecht angemacht (Hörzu 45, 1982, 16); Ich verdiene zurzeit gutes Geld, aber mein Job macht mich an (geht mir auf den Geist; Oxmox 7, 1985, 96). 2000 Dudenverlag

${ }^{32}$ an|feu|ern $<$ sw.V.; hat $>$ : 1. anzünden, anheizen: den Ofen, einen Herd, den Kessel a. 2. antreiben, anspornen: die Kämpfer, jmds. Mut a.; jmdn. zu immer größeren Leistungen a.; Bogatini... feuert das Orchester zu rasendem Fortissimo an (Thieß, Legende 196). 2000 Dudenverlag.

${ }^{33}$ strei|chen $<$ st. V. $>$ [mhd. strichen, ahd. strihhan, verw. mit Strahl]: 1. <hat $>$ a) mit einer gleitenden Bewegung [leicht, ebnend, glättend] über etw. hinfahren (3), hinstreichen: jmdm. freundlich, zärtlich, liebevoll durch die Haare, über den Kopf, das Gesicht s.; sie strich mehrmals mit der Hand über die Decke; er strich sich nachdenklich über den Bart/(auch:) strich sich den Bart; er streicht die Geige (veraltend; er spielt auf der Geige); Die Geiger strichen (spielten auf Streichinstrumenten) den Kammerton (Wellershoff, Körper 262); <nur im 2. Part.:> ein gestrichener (bis zum Rand gefüllter, aber nicht gehäufter) Esslöffel Mehl; das Maß sollte gestrichen voll (genau bis zum Rand gefüllt) sein; der Aschenbecher ist mal wieder gestrichen (ugs.; übermäßig) voll; Ü Der Strahl aus Schwester Noras Taschenlampe strich über die Ziegelmauer (Sebastian, Krankenhaus 86); wie eine Katze schnurret, wenn man sich mit ihr anlässt, ihr den Balg streicht (streichelt; Gotthelf, Spinne 94); b) mit einer streichenden ( 1 a) Bewegung irgendwohin befördern: mit einer raschen Bewegung strich sie die Krümel zur Seite, vom Tisch; ich strich ihm das Haar, eine Strähne aus der Stirn; mit einem Spachtel Kitt in die Fugen s.; Er nahm ihr das Brettchen mit den würflig geschnittenen Zwiebeln 


\section{Zusammenhang mit einem auch mehrdeutigen Substantiv Streifen ${ }^{34}$ gespielt, das in diesem Text nur im Sinne von den Mustern auf dem Fell des Zebras gebraucht wird.}

ab und strich sie mit dem Küchenmesser in die Sauce (M.L. Fischer, Kein Vogel 32); die gekochten Tomaten durch ein Sieb s. (Kochk.; durchschlagen, passieren). 2. $<$ hat $>$ a) mit streichenden (1 a) Bewegungen [mithilfe eines Gerätes] in einer Schicht über etw. verteilen, irgendwo auftragen: Butter, Marmelade [dick] aufs Brot s.; er strich mit dem Spachtel Salbe auf die Wunde; b) durch Streichen (2 a) mit einem Brotaufstrich versehen; bestreichen: ein Brötchen mit Leberwurst, Honig, Marmelade s.; der Vater streicht den Kindern die Brote; c) mithilfe eines Pinsels o. Ä. mit einem Anstrich (1 b) versehen; anstreichen: die Decke, die Wände s.; sie hat die Türen, die Gartenmöbel mit Ölfarbe gestrichen; Das Fenster musste gestrichen werden (H.Gerlach, Demission 270); ein grün, mit grüner Ölfarbe gestrichener Zaun; Vorsicht, frisch gestrichen! 3. (etw. Geschriebenes, Gedrucktes, Aufgezeichnetes) durch einen od. mehrere Striche ungültig machen, tilgen; ausstreichen $\langle$ hat $>$ : einen Satz [aus einem Manuskript, in einem Text] s.; er hat einige Passagen der Rede, einige Szenen des Theaterstückes gestrichen; du kannst sie, ihren Namen aus der Liste s.; Nichtzutreffendes bitte s.!; Es versteht sich, dass ich kein Wort gestrichen habe (Reich-Ranicki, Th. Mann 266); Ü du musst diese Angelegenheit aus deinem Gedächtnis s. (musst sie vergessen, darfst nicht mehr daran denken); Kann ich zehn Jahre aus meinem Leben s.? (sie als nicht da gewesen betrachten?; Seidel, Sterne 143); der Auftrag wurde gestrichen (zurückgezogen); dass ihm ein Kredit erst zugesagt und dann auf unerklärliche Weise wieder gestrichen worden sei (H.Weber, Einzug 78); Zuschüsse, Subventionen s. (nicht länger gewähren, abschaffen); dem Häftling sind alle Vergünstigungen gestrichen worden (werden ihm nicht länger gewährt); deinen Urlaub, diese Pläne kannst du s. (ugs.; aufgeben, fallen lassen); Der Computerhersteller ... soll innerhalb von zwei Jahren 40000 Stellen s. (abschaffen, abbauen; Tages Anzeiger 26.11. 91, 5). 4. <ist> a) ohne erkennbares Ziel, ohne eine bestimmte Richtung einzuhalten, irgendwo umhergehen; sich irgendwo umherbewegen: durch Wälder und Felder s.; abends streicht sie durch die Straßen, um ihr Haus; wenn er mit den Kindern durch die Gegend strich (Harig, Ordnung 19); die Katze streicht (bewegt sich mit leichten, gleitenden Bewegungen) mir um die Beine; b) (bes. Jägerspr.) in ruhigem Flug [in geringer Höhe] fliegen, irgendwo umherfliegen: ein paar Enten, Wasservögel streichen aus dem Schilf; Ein Flugzeug strich über die Wolken (Loest, Pistole 78); c) irgendwo gleichmäßig, nicht sehr heftig wehen: ein leichter Wind streicht durch die Kronen der Bäume, über die Felder, um die Mauern; Kühl streicht Morgenluft über meine Schultern (Lentz, Muckefuck 21); War der kühle Wind, der bei einem Morgenbad über das spiegelglatte Meer strich, schuld? (Gregor-Dellin, Traumbuch 101). 5. <in den Vergangenheitsformen ungebr.> a) (Geol.) (von schräg verlaufenden Schichten) in bestimmtem Winkel eine gedachte horizontale Linie schneiden; b) (Geogr.) (von Gebirgen) in eine bestimmte Richtung verlaufen, sich erstrecken: das Gebirge streicht nach Norden, entlang der Küste. 6. (Rudern) (das Ruder) entgegen der Fahrtrichtung bewegen od. stemmen, um zu bremsen od. rückwärts zu fahren $\langle$ hat $>$ : sie haben die Riemen gestrichen. 7. (Seemannsspr. veraltet) herunterlassen, einziehen, einholen $\langle$ hat $\rangle$ : die Segel, die Stenge s. 8. (landsch.) melken (1) <hat>. 9. verprügeln; züchtigen; schlagen $<$ hat $>$ : Wenn die Burschen schwimmen und ich seh einen nackten Buckel, gleich fallen sie mir zu Dutzenden ein, die ich habe mit Ruten s. sehn (Goethe, Egmont II); die Mutter ... nahm mich und strich mich eine lange Zeit mit der Rute (Rosegger, Waldbauernbub 178). 2000 Dudenverlag.

${ }^{34}$ Strei|fen, der; -s, - [mhd. strife, verw. mit Strahl; 2. gek. aus Filmstreifen]: 1. a) farblich von seiner Umgebung abgehobener langer, schmaler Abschnitt einer Fläche: ein silberner, heller S. am Himmel, Horizont; das Kleid hat breite, schmale, feine S.; er hat den weißen S. auf der Fahrbahn überfahren; *in den S. passen (ugs.; zu jmdm., etw. passen, mit seiner Umgebung, Umwelt harmonieren; wohl nach der Vorstellung der Abstimmung verschieden gemusterter Stoffe aufeinander): die neue Mitarbeiterin passt in den S.; jmdm. [nicht] in den S./[nicht] in jmds. S. passen [Kram 20]). 2000 Dudenverlag. 


\section{(33) Zebra}

Ist eine Blondine im ZOO, sieht sie am Zaun vom Zebragehege ein Schild ,frisch gestrichen'. ,Oh!' sagt sie: "ich dachte die Streifen wären echt!"

Die Situationen mit den Blondinen am Steuer werden oft zum Thema der Witze, das man auch mit Hilfe der zur Verfügung stehenden sprachlichen Mittel im Rahmen eines kurzen Frage-Antwort-Witzes präsentiert, z.B.

\section{(34) Autounfall mit Blondine}

Eine Blondine hat mit ihrem Auto ein anderes Fahrzeug gerammt. Brüllt der Fahrer:

"Sie dummes Huhn, haben Sie überhaupt eine Fahrprüfung gemacht?"

Zischt die Blondine zurück: „Bestimmt öfter als Sie!”

Im zitierten Witz wird mit der Doppeldeutigkeit der Wortgruppe eine Prüfung machen gespielt, wobei man in diesem Falle sogar die beiden Lesarten einsetzt, d.h. die wortwörtliche Bedeutung von 'an der Prüfung teilnehmen' wird im Zusammenhang mit der Blondinen-Figur und die von 'eine Prüfung bestehen' mit dem Mann gemeint.

An dieser Stelle soll noch ein weiteres Beispiel genannt werden, in dem der Sinn der Aussage deutlich mit der Polysemie des Wortes Test im Determinativkompositum Schwangerschaftstest ${ }^{35}$ zusammenhängt und für den Rezipienten nur eine richtige Variante impliziert, wobei es von den Witzfiguren selbst im Sinne von 'eine Art schriftliche Prüfung' verstanden wird

\section{(35) Schwangerschaftstest}

Zwei Blondinen unterhalten sich. Sagt die eine: "Ich machte einen Schwangerschaftstest".

Darauf die andere: "Und, waren die Fragen schwer?"

${ }^{35}$ Test, der; $-[\mathrm{e}] \mathrm{s},-\mathrm{s}$, auch: $-\mathrm{e}$ [engl. test, eigtl. $=3$ Kapelle $<$ afrz. test $(>$ mhd. gleichbed. test $)=$ Topf (für alchemistische Versuche) < lat. testum, zu: testa= Platte, Deckel; (Ton)schale, Scherbe]: nach einer genau durchdachten Methode vorgenommener Versuch, Prüfung zur Feststellung der Eignung, der Eigenschaften, der Leistung o.Ä. einer Person od. Sache: ein wissenschaftlicher, klinischer, psychologischer T.; 2000 Dudenverlag Schwan|ger|schafts|test, der: Test zur Feststellung einer Schwangerschaft. 
Interessant ist die Tatsache, dass die Witze einen internationalen Charakter haben können, was man u.a. am Beispiel des zitierten Textes sagen kann, der auch auf Polnisch zu finden ist und eine 1:1 Entsprechung ist, was wiederum mit der Bedeutung des Wortes test selbst im Zusammenhang steht, das als Internationalismus zu verstehen ist, wegen seiner fachbezogenen Bedeutung:

(35*) Rozmawiaja dwie blondynki. Jedna mówi „Wiesz, zrobiłam sobie test ciażowy", na to druga "No i co? Trudne były pytania?"36

Im Polnischen entspricht dem Determinativkompositum eine Wortgruppe Substantiv+Adjektiv test ciażowy.

Mit dem Begriff des Wortspiels werden dann weitere Begriffe assoziiert [Detering 1983; Fiedler 2003; Ulrich 1976, 2007; Tęcza 1997] ${ }^{37}$, d.h.

- Sprachscherz

- Sprachspiel

- Sprachspielerei

- Sprachwitz.

Den Definitionen zufolge scheint es eindeutig zu sein, dass es ein kreativer und absichtlicher Vorgang sein soll. Der Erzähler oder Textautor bedient sich seiner Sprache bewusst, was auch mit der Kenntnis aller versteckten Inhalte und Nuancen des jeweiligen Sprachsystems auf allen seinen Ebenen verbunden ist. Er nutzt dieses Wissen aus, um humoristische Texte zu schreiben, indem er seinen Figuren bestimmte Idiolekte und Soziolekte zuteilt, sie auf eine bestimmte Art und Weise ihre Aussagen formulieren lässt, die nicht selten voll von verschiedenen fehlerhaften Elementen sind, was den humoristischen Effekt noch verstärkt. Die Figuren sprechen meist die Umgangssprache, wenn sie Fehler begehen, dann sind es Schwierigkeiten mit der fachbezogenen Lexik oder mit den fremdsprachigen Elementen. Wegen der Eigenschaften, die von den Autoren der jeweiligen Figur zugeschrieben werden und die sich in den meisten Fällen auf deren fehlende Intelligenz bzw. das gar nicht vorhandene Wissen aus einem bestimmten Bereich oder

${ }^{36}$ Vom Hören-Sagen bekannt

${ }^{37}$ Laut DUDEN ist Sprach|spiel, das [eingef. von dem österr. Philosophen L.Wittgenstein (1889-1951)] (Sprachw.): Verwendung von Sprache, sprachliche Tätigkeit als Teil einer Tätigkeit od. Lebensform od. im Zusammenhang mit einer anderen beliebigen Handlung. 2000 Dudenverlag; Sprach|spie|le|rei, die: Spielen mit der Sprache unter Nutzung von Mehrdeutigkeiten, lautmalenden Klängen, Wortspielen o.Ä. 2000 Dudenverlag. 
sogar auch das fehlende Allgemeinwissen zurückführen lassen, werden für die Zwecke des Humoristischen solche sprachlichen Mittel eingesetzt, mit denen das alles besonders scharf ausgedrückt und eindeutig gezeigt werden kann.

Mit dem Sprachspiel wird vor allem der kreative Umgang mit der jeweiligen Sprache verstanden, wobei es um die Wortspiele, Spiele mit der Wortbildung, Reime und Alliterationen oder auch um die Anspielung geht. Dieser Begriff wird von Wittgenstein [1989, Bd. 1] im Rahmen der von ihm formulierten Auffassung der Sprachspiele und der damit zusammenhängenden Gebrauchstheorie der Bedeutung genannt. Wittgenstein nennt in seinen Arbeiten zahlreiche Beispiele der Ebenen, auf denen das Sprachspiel entstehen kann, unter denen er auch den Witz platziert - den Text selbst und sein Erzählen. Wittgenstein betont auch das Wesen des Sprachspiels als Summe aller sprachlichen Elemente und bespricht ihre Wirkung auf den Rezipienten. Dem Sprachspiel wird auf diese Weise eine Funktion zugeschrieben, die erst in Verbindung mit allem Außersprachlichen sichtbar ist. Die Umstände, in denen eine lustige Geschichte produziert und vorgetragen wird, entscheiden im Endeffekt über ihre Interpretation, denn sie wirken in dem gleichen Maße auf den Rezipienten und beeinflussen seine Wahrnehmung des Erzählten. Die Körpersprache betont die sprachlich ausformulierte Aussage so, dass man konkrete Absichten des Gesprächspartners sofort erkennt und nach seiner Erwartung handelt - im Falle der scherzhaften Kommunikation ist es das Gelächter.

Es darf dabei aber nicht außer Acht gelassen werden, dass es innerhalb eines Sprachsystems „normale“, also konventionelle und konventionalisierte Wörter gibt und dass neben ihnen auch die sog. ,,anormalen“ zu finden sind, die wegen ihrer unklaren Relation zu der außersprachlichen Welt verschiedene Interpretationen zulassen. Es liegt u.a. dann vor, wenn ein Wort z.B. eine neue Bedeutung erfährt und in einem alten, bisher geltenden Kontext gebraucht wird, wie es im Falle eines aus dem Deutschen entlehnten Wortes sein kann, z.B. das Lexem fladra im Polnischen als 'eine alte, geschwätzige Frau', das aber von dem Rezipienten in der Grundbeutung, also als 'Plattfisch' verstanden wird oder landara, was im Polnischen als pejorative Bezeichnung für 'eine unsympathische, dicke Frau' verwendet wird und nur die Verschiebung der Grundbedeutung der deutschen Vorlage 'klobige Kutsche' ist. ${ }^{38}$

Im Rahmen der polnischen Humorforschung ist Buttler [2001] das größte Verdienst zuzuschreiben, die das Polnische unter dem Aspekt des Humoristischen analysierte. Sie betont die Funktion der Sprache innerhalb der scherzhaften Kommunikation und weist auch darauf hin, dass es neben den reinen sprachlichen

${ }^{38}$ Mit zahlreichen solchen Beispielen habe ich mich in meiner Dissertation befasst: Sikorska [2001]. 
Witzen auch solche gibt, die nicht nur aus der Sprache schöpfen, sondern auch die Mechanismen des Komischen so ausnutzen, dass sie die erwartete Reaktion der Rezipienten hervorrufen [Ebenda, S. 68-69].

Bei der Definition des Wortspiels spielt vor allem ein Faktor die entscheidende Rolle - die Absicht des Sprechers, woraus resultiert, dass fehlerhafte Normen, die zufällig dem Sprechenden unterlaufen, vor allem wegen ihrer Zufälligkeit, nicht als Wortspiele zu klassifizieren sind.

Es kommt nicht selten vor, dass die Bezeichnungen Sprachspiel und Wortspiel abwechselnd gebraucht werden [Grassegger 1985; Poethe 2002; Müller 2002; Ulrich 2007; Garski 2008]. Heibert [1993, S. 19] erklärt, wie ein Wortspiel zu verstehen sei, wenn es als ein so genanntes absichtliches Missverständnis zustande kommt und betont, dass die Polysemie und Homonymie die gleiche semantische Situation betreffen:

Die geläufige Vorstellung, ein Wortspiel ist so etwas wie ein absichtliches Missverständnis, das auf der Mehrdeutigkeit von Wörtern beruhe, bezieht sich auf jenen Wortspieltyp, der zwei homonyme Lexeme miteinander verknüpft. Es gibt aber auch solche, die mit Syntagmen, also Lexemsequenzen spielen (mit Redensarten, Sprichwörtern), welche als ganzes zum Sprachbesitz gehören.[...] Die Wortspielanalyse behandelt [...] Wortspiele mit homonymen Lexemen und solche mit doppeldeutigen Lexien [...] als Varianten der gleichen semantischen Situation.

An dieser Stelle muss noch einmal betont werden, dass das Wortspiel dem Sprachspiel untergeordnet ist. Die sprachlichen Spiele werden so realisiert, dass entweder mit den Wörtern, Wortpaaren, Wortgruppen oder mit den ganzen Sätzen absichtlich so gespielt wird, dass es aufgrund der erzeugten Doppeldeutigkeit zu den Missverständnissen kommt, was im Rahmen der scherzhaften Kommunikation geschickt ausgenutzt wird. Es muss dabei auch darauf aufmerksam gemacht werden, dass auch die Wortbildungsmodelle den Ausgangspunkt für die komischen Elemente sein können, wo man z.B. im Deutschen mit den Komposita spielt, indem man einfache Lexeme nach dem Modell des Determinativkompositums zu erklären versucht, z.B. Stahl vs. Diebstahl im weiteren Teil dieser Arbeit zitierten Witz (39). In erster Linie werden die Wortspiele in zwei Gruppen eingeteilt - die im weiteren und im engeren Sinne [Tęcza 1997]. Im ersteren Fall geht es um eine solche Relation zwischen dem Ausdruck und dem Inhalt, dass einem Lexem mindestens zwei Varianten zugeschrieben stehen, die zu den Missverständnissen führen können, wie es im Falle der bereits zitierten Witze ist, z.B. surfen, Test, anmachen oder anfeuern, in dem letzteren dagegen darum, dass entweder Neologismen gebildet werden oder dass man gegen morphosyntaktische Normen verstößt und falsche Formen für die humoristischen Zwecke gebraucht oder aber dass die 
aus einer anderen Sprache entlehnte Lexik in einem falschen Kontext erscheint, so dass somit komische Effekte erzielt werden können [Janicka 2011]. Die Normverstöße jeder Art können demzufolge als Mittel des Humoristischen dienen und werden den Wortspielen im weiteren Sinne zugeordnet.

Neben dieser Klassifikation gibt es auch eine andere, in der von zwei Kategorien der Wortspiele die Rede ist - von den textimmanenten und den kontextuellen Wortspielen.

Als textimmanente Wortspiele werden Spiele solcher Art betrachtet, die aus der Funktion der sprachlichen Mittel innerhalb eines konkreten Textes resultieren und die kontextuellen sind dagegen diese, die erst aus der Relation zu den Kontextpartnern zu entschlüsseln sind. Dabei ist aber auch darauf hinzuweisen, dass nicht nur der sprachliche, sondern auch der situative Kontext zu beachten ist. Das ist besonders im Witz (30) Surfen von Bedeutung, denn erst der in der Pointe genannte situative Kontex, also die Tatsache, dass Thomas keinen Computer hat, bewirkt eine bestimmte Reaktion des Rezipienten, der auf einmal mit etwas Unerwartetem konfrontiert wird, so dass somit die Spannung gelöst wird und der Inhalt des ganzen Witzes mit dieser notwendigen Information einerseits ergänzt und andererseits in eine andere Richtung der Interpretation gelenkt wird. Das ganze Feld von Assoziationen, das für jeden Kommunikationspartner einzigartig und nur für ihn charakteristisch ist, lässt nämlich zahlreiche Interpretationen eines und desselben Textes zu. Wenn mit den textimmanenten Spielen oft in der Werbung [Sauer 1998] manövriert wird, werden für die Zwecke der humoristischen Texte die kontextuellen Spiele ausgenutzt.

Nach Hausmann [1974] wird zwischen den vertikalen und horizontalen Wortspielen unterschieden. Die ersteren liegen dann vor, wenn einem Ausdruck zwei verschiedene Bedeutungen zugeschrieben stehen, von denen die eine die wörtliche und die andere die übertragene Bedeutung ist, so wie es im Falle von etw. gegen etw. haben im Sinne von 1.'etw. nicht wollen', 2.'etw. gegen etw. Wirkendes haben' und zum Schluss noch 3.'etw., was das Empfinden von etw. mildert oder sogar verdrängt', wie im Satz z.B. Haben Sie etwas gegen Kopfschmerzen?, der verschieden interpretiert werden kann, d.h. als Frage in der Apotheke 'Haben Sie Tabletten gegen Kopfschmerzen?' oder als eine ziemlich seltsame Frage 'Mögen Sie Kopfschmerzen nicht?', die selten verneint wird. Auf diese Weise entsteht die Doppeldeutigkeit. Ein und dassselbe Wort bezieht sich in diesem Kontext auf mindestens zwei verschiedene Sachverhalte.

Wenn in einem Satz zwei gleiche oder ähnliche Wörter auftauchen und sich auf einen und denselben Sachverhalt beziehen, dann liegt ein horizontales Wortspiel vor. Hausmann [Ebenda] bedient sich des Beispiels von natürlich, wo zwei verschiedene Bedeutungen genannt werden müssen, d.h. 1.'selbstverständlich' 
und 2.'echt, nicht künstlich, naturbelassen', Fältchen kann man natürlich akzeptieren. Oder auf natürliche Weise mindern.

Im Rahmen der humoristischen Texte werden von den Autoren in den meisten Fällen vertikale Spiele gebraucht, vor allem deswegen, weil sie schneller zu den Missverständnissen führen können. Es kommt vor, weil die Autoren höchstwahrscheinlich davon ausgehen, dass einem Rezipienten die eine oder andere Bedeutungsvariante der für die humoristischen Zwecke gebrauchten Lexik nicht bekannt sein kann und sogar soll, damit eben das Komische entstehen kann.

\subsection{Polysemie, Homonymie und Homophonie als Mittel des Humoristischen}

Wie schon mehrmals betont wurde, spielen in der scherzhaften Kommunikation vor allem die Polysemie, die Homophonie und das Wortspiel eine wichtige Rolle. Die Autoren greifen gezielt zu diesen Mitteln wegen ihrer Bildhaftigkeitsfunktion in den Witzen, die sie schreiben. Der Sprachökonomie zufolge kann auf diese Weise ein relativ reicher Inhalt mit möglichst wenig Mitteln zum Ausdruck gebracht werden. Diese Mehrdeutigkeit hängt aber nicht nur mit der sprachlichen Seite eines witzigen Textes, sondern auch mit dem außersprachlichen Kontext zusammen und betrifft somit auch die Körpersprache [Passi 1980], die sich aus verschiedenen Gesten, Körperbewegungen und aus der Mimik zusammensetzt. Interessant kann dabei aber auch eine andere Erscheinung sein, wenn das Komische ganz zufällig zustande gebracht wird, vor allem dann, wenn der Erzählende selbst eine oder andere Bedeutungsvariante des gebrauchten polysemen Wortes nicht kennt [Bystroń 1960; Buttler 1936]. Der Witz, für den man vor allem zu der Polysemie als zu diesem sprachlichen Mittel greift, das das größte Bedeutungspotenzial hat, gilt als die typischste Form des Wortwitzes.

Polyseme oder mehrdeutige Wörter [Bußmann 1990, S. 593] werden in erster Linie metaphorisch gebraucht, was auf ihre gemeinsamen gleichen oder ähnlichen Bedeutungsmerkmale zurückzuführen ist [Sikorska 2001, S. 44-45]. Demzufolge stehen u.a. dt. Bein und poln. noga als Bezeichnungen für 'Körperteil' und (Tisch) bein und noga (stolu) 'Teil eines Gegenstands', dagegen ist koński ząb 'Zahn des Pferdes' im Sinne von 'Mais' zu verstehen. Die Relation von 'Tierbezeichnung' zu 'Körperteil des Tieres' und die damit verbundene Übertragung der Bezeichnung sind somit sichtbar. Dies betrifft auch die Beziehungen: 'Mensch' und 'Tier' (dt. Ameise vs. poln. mrówka als Bezeichnung eines tüchtigen Menschen) oder 'Mensch' und 'Mensch' (dt. Xanthippe vs. poln. Ksantypa als 'streitsüchtige Frau'). 
Eine andere sprachliche Erscheinung, die mit der Übertragung der Begriffe aus einem Sinnbereich auf einen anderen zusammenhängt ist die Synästhesie [Mitempfindung; Bußmann 1990, S. 760], z.B. kalte Augen als 'keine Emotionen ausstrahlende Augen', seidiges Haar 'sehr weiches, feines, der Seide ähnliches Haar', eiserner Blick 'harter Blick', poln. zimne spojrzenie für 'kalter, tadelnder Blick' oder poln. kamienna twarz 'Gesicht, von dem man keine Emotionen ablesen kann', politisches Klima 'politische Situation' oder poln. zamrozić ceny 'Preise einfrieren' und Preise einfrieren usw.

Laut Lexikonsdefinitionen wird Polysemie als ,,das Vorhandensein mehrerer Bedeutungen bei einem Wort" [GD 1165] verstanden, Homonymie dagegen - als ,,lautliche Übereinstimmung von Wörtern mit verschiedener Bedeutung [u. Herkunft]" [GD 734]. Dabei wird auf die Grenzen zwischen ihnen hingewiesen, die nach folgenden Kriterien unterschieden werden [Schippan 1972, S. 90ff.]

- etymologische

- semantische

- orthographische

- morphosyntaktische

Jedoch muss an dieser Stelle betont werden, dass eine eindeutige Abgrenzug der Polysemie und der Homonymie nicht möglich ist [Sikorska 2001]. Nach dem etymologischen Kriterium wird die Lexik diachronisch analysiert und somit kann das genannte Kriterium nicht bei der synchronen Untersuchung beachtet werden. Demzufolge werden polyseme Wörter als die Bedeutungsvarianten definiert, die auf dieselbe Wurzel zurückgehen, wohingegen die Homonyme in der Regel mit keiner Art Relation zwischen den Bedeutungsvarianten eines und desselben Wortes zusammenhängen. „, Alle Bedeutungsvarianten eines polysemen Wortes hängen zusammen, weil sie durch gemeinsame semantische Merkmale miteinander verbunden sind." [Bondzio 1980, S.109]. So ist das Merkmal [+Vorwärtsbewegung] das Gemeinsame für das polyseme Verb laufen [Ebenda, S. 109f.], dessen Lesarten durch das Hinzukommen der weiteren semantischen Eigenschaften ([+zu Fuß], [+räumlich begrenzt], [+zeitlich begrenzt] usw.) differenziert werden.

Syntaktische Gebrauchsregeln tragen dazu bei, dass die Monosemierung zustande kommt. Erst der Kontext löst die Mehrdeutigkeit eines polysemen Wortes auf und somit wird das jeweilige Wort eindeutig. So kann Fuchs als polysemes Substantiv in folgenden Varianten vorkommen [GD 545]:

1. 'kleineres Raubtier mit rötlichbraunem Fell, spitzer Schnauze, spitzen Ohren und buschigem Schwanz'

2. a.'Fell des Fuchses', b.'aus dem Fuchsfell angefertigter Pelzmantel' 
3. (abwert.) 'listiger Mensch'

4. (ugs., meist abwert.)'Mensch mit roten Haaren'

5. 'Pferd mit rötlichbraunem Fell'

6. 'Tagfalter mit gelb- bis rotbraunen, blau bis schwärzlich gefleckten und gesäumten Flügeln'

7. (Studentensprache) 'noch nicht vollberechtigtes Mitglied einer Studentenverbindung im ersten und zweiten Semester'

8. (veraltet) 'Goldmünze'

9. 'Abzugskanal einer Feuerung zum Schornstein'

Erst im Kontext wird die Polysemie von Fuchs aufgelöst und das Wort tritt nur in einer seiner zahlreichen Bedeutungsvarianten auf, z.B. 1. Er reitet einen Fuchs. impliziert die Lesart (5) des genannten Wortes, 2. Frau Müller lässt sich einen Fuchs nähen. hängt mit Fuchs (2.b.) zusammen bzw. 3. Er ist ein echter Fuchs, denn er ist schlauer als wir alle. betrifft Fuchs in der Bedeutungsvariante (3). Die Mehrdeutigkeit soll vor allem als eine lexikalische, den sprachlichen Zeichen zugeschriebene Eigenschaft definiert werden. Der aktuelle Gebrauch des jeweiligen mehrdeutigen Lexems bewirkt die Auflösung der ganzen Reihe von Bedeutungsvarianten zugunsten nur einer Bedeutung. Der Kontext, in dem das bisher mindestens doppeldeutige Wort gebraucht wird, bewirkt es, dass das Lexem disambiguiert ${ }^{39}$ wird. Die im Kontext genannten semantischen Merkmale passen nur zu einer der alternativen Bedeutungsvarianten des mehrdeutigen Lexems, so dass demzufolge die anderen nicht kompatibel sind und zurücktreten müssen, wie es am Beispiel des folgenden Blondinen-Witzes gezeigt werden kann, in dem die Ambiguität des Lexems scharf ausgenutzt wird:

\section{(36) Blondine und Fernseher \\ Warum streut eine Blondine Pfeffer auf ihren Fernseher? Damit das Bild schärfer wird!}

In dem genannten Kontext geht es um das Adjektiv scharf und seine Variante 'deutlich [+sichtbar], klar [+hervortretend], nicht verschwommen', der von der Witzfigur die Lesart 'stark, intensiv' gegenübergestellt wird, was im Endeffekt das Humoristische erzeugt.

${ }^{39}$ dis $\mid$ am $\mid$ bi|gu|ie|ren <sw.V.; hat $>$ [zu lat. dis-= un-, nicht u. ambiguus, ambig] (Sprachw.): die Ambiguität eines sprachlichen Zeichens, eines Ausdrucks (durch bestimmte syntaktische od. semantische Zuordnungen) aufheben; etw. eindeutig machen. 2000 Dudenverlag. 
Im Falle der gesprochenen Witze, deren Texte dem Rezipienten nur auf dem Wege des Gesagten präsentiert werden, kommt es auch dazu, dass sich die Mehrdeutigkeit auf die phonetische Ähnlichkeit bezieht und auf die Homophonie zurückzuführen ist, z.B. im Falle des Verbs modern als 'faulen, faul werden 'und des Adjektivs modern 'modegerecht'.

Wenn die beiden Lexeme nicht in einem Kontext schriftlich gegenübergstellt werden, dann kommt es dazu, dass das Wortpaar selbst auch nicht ausreicht, damit die phonetische Mehrdeutigkeit gelöst wird. Es müssten dann zusätzliche Informationen genannt werden. Diese Erscheinung taucht oft in den Sketchen auf, vor allem deswegen, weil diese auf der Bühne präsentiert werden, also ohne dass der Rezipient sie dank dem sprachlichen Kontext identifizieren und richtig entschlüsseln kann.

Demzufolge wird dem Sprecher und dem Hörer als Kommunikationspartner eine besondere Funktion zugeschrieben, nämlich eine solche, dass sie aufgrund ihres Wissens die gehörten bzw. gelesenen sprachlichen polysemen Elemente monosemieren sollen, was mit der Entschlüsselung und der Interpretation der gesagten bzw. gehörten Lexeme und ihren Bedeutungen zusammenhängt. Von dem Rezipienten wird im Falle der humoristischen Texte verlangt, dass er über ein ausreichendens Vorwissen verfügt, der jeweiligen Sprache samt ihren Nuancen auf allen Ebenen mächtig ist, mindestens gute Englischkenntnisse vorzuweisen hat und zu guter Letzt noch - viel Sinn für Humor hat, der ihm erlaubt, alles Lustige komisch zu finden und alles Humoristische, was von den Autoren versteckt, verschlüsselt und zwischen den Zeilen gesagt wird, zu finden, alle damit verbundenen Absichten der Texter zu erkennen, zu verstehen und demzufolge den Text als eine Einheit zu interpretieren. Der Rezipient wirkt an jedem Sketch mit, weil er die Kontexte mitproduzieren soll, was aus der Ambiguität der sprachlichen und der außersprachlichen Kontexte resultiert und auf die mindestens zwei Skripts der jeweiligen Witztexte zurückgeht. Das Assoziationsfeld um jedes polyseme Wort herum lässt nicht nur eine Interpretation zu, sondern überlässt dem Zuhörer bzw. Zuschauer die Entscheidung, wie die von ihm gehörten bzw. gelesenen Texte wirken, d.h. ob sie tatsächlich aufgrund der Ambiguität der dort auftauchenden Elemente als lustig einzustufen sind. Natürlich darf dabei nicht außer Acht gelassen werden, dass die Wahrnehmung der Witze auch von der Sensibilität jedes einzelnen Menschen abhängt, die ihm entweder erlaubt oder aber auch verbieten kann, über bestimmte Sachen zu lachen, weil diese für ihn nicht in die Kategorie der Witze gehören, wenn sie u.a. alte Menschen, ihre Krankheiten, den Tod, sexuelles Leben usw. thematisieren.

Das erwähnte semantische Kriterium betrifft die Homonyme, die dieselben grammatischen Merkmale haben, z.B. der Kater als 1.'männliche Katze' und 
2.'Zustand nach Alkoholgenuß' oder das Futter als 1.'Nahrung für Tiere' und 2.'untere Stoffschicht' bzw. warten als 1.'harren' und 2.'behüten' usw. Das letzte Kriterium - das orthographische hängt mit dem Signalisieren unterschiedlicher Wörter zusammen, ohne dass auf ihre Etymologie bzw. Semantik bzw. ihre grammatischen Eigenschaften eingegangen wird

Als Ausgangspunkt für die Unterscheidung der Homonyme sind nach dem morphosyntaktischen Kriterium die Unterschiede im Bereich der grammatischen Kategorien (Genus und Numerus bei den Substantiven, Grundformen bei den Verben) zu verstehen. Es können entweder die etymologisch verwandten (der Band 'Buch'/ das Band 'Stoffstreifen'< binden) oder etymologisch nicht zusammenhängenden Homonyme [Schippan 1972, S. 91] genannt werden (die Mark 'Währungseinheit' / das Mark 'Inhalt der Knochen', der Kiefer 'Schädelknochen'/ die Kiefer 'Nadelbaumart', der Tau 'Niederschlag'/ das Tau 'Seil' usw.). Des weiteren wird die unterschiedliche Pluralbildung als Merkmal der Homonyme verstanden, z.B. das Wort 'Vokabel' - die Wörter und das Wort 'jds Aussage' - die Worte; das Band 'Stoffstreifen' - die Bänder und der Band 'Buch' - die Bände usw. In die Kategorie der Homonyme fallen auch Verben mit unterschiedlichen grammatischen Formen, d.h. ihre Infinitiv- und Präsensform sind gleich, aber die Formen des Präteritums und des Partizip II. und ihre Bedeutungen unterscheiden sich voneinander, z.B. hängen - hing - gehangen vs. hängen - hängte - gehängt, quellen - quoll - gequollen vs. quellen - quellte - gequellt usw. Als homonyme Wörter können auch gleichlautende Vertreter verschiedener Wortarten betrachtet werden, z.B. morgen vs. der Morgen, rot vs. das Rot, essen vs. das Essen usw. „Damit ist mit der Wortart auch die Einheit in eine semantische Kategorie eingeordnet, so daß wir berechtigt sind, davon zu sprechen, daß wir es mit zwei Wörtern und nicht mit einem polysemen Wort zu tun haben." [Ebenda, S. 91].

Ein besonderer, auch in den witzigen Bühnetexten gebrauchter Typ der Homonymie ist die Homophonie. Laut DUDEN ist ein homophones ${ }^{40}$ Wort ,, Wort, das mit einem anderen gleich lautet, aber verschieden geschrieben wird (z.B. Lehre: Leere)".

Im Rahmen des Humoristischen werden Homophone vor allem in den Sketchtexten gebraucht, denn nur auf dem phonetischen Wege kann diese Ei-

${ }^{40}$ Ho|mo|phon, das; -s, -e (Sprachw.): Wort, das mit einem anderen gleich lautet, aber verschieden geschrieben wird (z.B. Lehre Leere); ho|mo|phon $<$ Adj. $>$ [griech. homóphonos $=$ gleich klingend, übereinstimmend]: 1. (Musik) in der Kompositionsart der Homophonie, wobei die Melodiestimme durch Akkorde gestützt wird u. die Stimmen weitgehend im gleichen Rhythmus verlaufen: eine Komposition in -em Satz, -er Schreibweise. 2. (Sprachw.) (von Wörtern od. Wortsilben) gleich lautend: die vier -en Wortsilben in zweieinhalb Zeilen verletzen... das... Sprachgefühl (Deschner, Talente 334) 2000 Dudenverlag. 
genschaft der lautlichen Ähnlichkeit ausgenutzt werden und zu den erwarteten Spielen mit der Sprache führen. Homophone Elemente führen leicht zu den Verwechslungen, erst in der Pointe stellt sich heraus, welches Lexem eines homophonen Paares gemeint wurde, z.B. poln. szef 'Chef' oder szew 'Naht'. Dabei wird noch die Bedeutung des polnischen Verbs krzywić się in seinen zwei Varianten gebraucht - der von 1.'langes Gesicht machen, Gesicht verzerren' und 2.'nicht gerade sitzen, liegen' in Bezug auf Strumpfhose mit Naht:

(37) Co to jest - krzywi się w biurze i na nodze? Szef / szew. ${ }^{41}$

oder im Deutschen, wenn z.B. ein homophones Paar - das Lexem Uhr und das Morphem $\underline{U}$ - gebraucht wird. Die Wortbildung des Deutschen lässt nämlich die Ad-hoc-Komposita zu (Uhrwald) und gibt dann auch die Möglichkeit, gezielt zu solchen Mitteln im Rahmen der scherzhaften Kommunikation zu greifen.

\section{(38) Was machen die Elefanten, wenn sie wissen wollen, wie spät es ist? \\ Sie gehen zum Urwald!}

Auch die Bildung per analogiam kann für die humoristischen Zwecke gebraucht werden, indem in einem witzigen Text das deutsche Kompositum Diebstahl falsch eingestuft, jedoch absichtlich eingesetzt wird, wenn das Material Stahl im Kompositum Diebstahl erscheint

(39) Woraus wird ein deutsches Auto gebaut? - Aus deutschem Stahl. Woraus wird ein schwedisches Auto gebaut? - Aus schwedischem Stahl. Und woraus wird ein polnisches Auto gebaut? - Aus Diebstah!

Unter den Witzen, in denen die Homophonie ausgenutzt wird, gibt es auch solche, wo man mit den Eigennamen spielt, z.B. Freitag im Sinne von ` fünfter Tag der mit Montag beginnenden Woche' und als Eigenname eines Romanhelden von Dafoe „Robinson Crusoe“

${ }^{41}$ „Was ist das - macht langes Gesicht/liegt nicht richtig im Büro und am Bein?“ - „Chef/ Naht") (eigene Übersetzung). 


\section{(40) Was haben Robinson Crusoe und Beamte gemeinsam? Beide warten auf Freitag}

Eine ähnliche Erscheinung kann im Falle des nächsten Witzes festgestellt werden, in dem die Ähnlichkeit des von einem Frosch produzierenden Geräusches Quaaaaak und der Bezeichnung eines Milchproduktes Quark zur Verwechslung führt:

\section{(41) Kommt ein Frosch in einen Milchladen. "Was wünschen Sie?", fragt der Verkäufer "Quaaaaak!"}

Diese Art Humor war der Ausgangspunkt zur Herausbildung der Wortspiele und der Witze selbst. Die wichtigsten Hinweise für die Analyse der polysemen witzigen Texte nennt schon Buttler [1968, 2001], die sich auf Bühler [1934] beruft und darauf zeigt, dass es wegen der in diesen Texten gebrauchten mehrdeutigen Lexik auch Schwierigkeiten beim Übersetzen geben kann, was auf die für jedes Sprachsystem charakteristische Struktur zurückzuführen ist, die sich u.a. in der Bedeutungsstruktur der vorhandenen Lexik manifestiert. Jedes einzelne Element des Wortschatzes hängt in gewissem Maße mit seinen Nachbarn im Wortfeld zusammen, das um ein bestimmtes Thema gebildet wird und alle Relationen innerhalb der Lexik beeinflusst, so dass die Lexeme verschiedene paradigmatische und syntagmatische Bedeutungsrelationen ${ }^{42}$ untereinander eingehen. Die Lexik besteht aus vielen Teilausschnitten - Wortfeldern, die eine zusammenhängende Einheit bilden. Ein Wort als Element der Lexik ist nicht als Einzelwort zu verstehen, sondern ist ein Teil einer semantisch komplementären Gruppe, die von ihm und seinen begrifflichen Nachbarn gebildet wird. ${ }^{43}$

Es kann am Beispiel eines kurzen Satzes Er ist ein Gesandter, aber kein Geschickter. gezeigt werden, wo einerseits mit der Polysemie der substantivisch gebrauchten partizipialen Formen der Verben schicken und senden und andererseits mit der Homophonie der Lexeme geschickt als 'gesandt' und geschickt im Sinne von 'wendig, gewandt' und gleichzeitig mit den Substantiven der Gesandte ' der Abgeordnete' und Geschickter 'eine geschickte, gewandte Person', das ein substantivisch gebrauchtes Adjektiv in der Bedeutung 'wendig, gewandt' ist, gespielt wird. Diese Vermischung der Formen beeinflusst das Entstehen des

${ }^{42}$ Dazu haben sich Porzig, Coseriu u.a. geäußert

${ }^{43}$ Darüber habe ich in meiner Dissertation geschrieben, wo ich mich mit der Bedeutungsveränderung der entlehnten Lexik befasst habe [Sikorska 2001]. 
komischen Kontextes, aber man muss alle sprachlichen Nuancen kennen, um den versteckten Sinn zu finden und diesen dann auch zu entschlüsseln. Ein anderes Beispiel dafür wären hier auch Witze aus dem Bereich des schwarzen Humors [Millowitsch 1970]

(42) Ein Mann besucht seinen sterbenden Freund.

"Ich leide Höllenqualen", ächzt der und wirft sich auf seinem Lager herum.

"Jetzt schon?" fragt der Freund

Die Bedeutung von Höllenqualen leiden, also 'sehr starke Schmerzen empfinden', wird hier mit der wortwörtlichen Bedeutung von Hölle 'Ort der ewigen Verdammnis für die Sünder; Reich des Teufels' vermischt. Der situative Kontext erlaubt der Witzfigur eine solche Bemerkung, denn ein im Sterben liegender Freund lebt noch, also es kann demzufolge keine Rede von der Hölle sein

(43) "Wenn ich sterbe”, sagt der Mann nachdenklich, "möchte ich verbrannt werden"

"Typisch für dich", sagt seine Frau gehässig. "Dann werde ich auch einmal die Asche im ganzen Haus verstreuen"

In diesem Fall geht es um das Spiel mit dem Wort Asche als 'staubig-pulveriger Rückstand verbrannter Materie' und der Wendung Asche verstreuen 1. 'streuend verteilen' oder 2.'versehentlich ausstreuen, verschütten' oder aber 3.' unachtsam, unordentlich an verschiedenen Stellen ablegen'. Die Frau reagiert mit Wut auf die Äußerung des Mannes wegen seiner schlechten Gewohnheit, die Zigarettenasche im ganzen Haus zu verstreuen. Es wird von ihr aber eine ganz andere Reaktion erwartet, die mit der Bedeutung von Asche als die der verbliebenen staubig-pulverigen Rückstände des verbrannten menschlichen Körpers zusammenhängt. Die Pointe erfüllt demzufolge ihre überraschende Funktion.

(44) "Stellen Sie sich vor", weint Frau Meier, "mein Mann ist von einer Dampfwalze überfahren worden und liegt im Städtischen Krankenhaus."

"Grauenhaft", ruft die Freundin aus. "In welchem Zimmer liegt er denn?"

"Zimmer dreizehn bis achtzehn" 
Wie in den früheren Texten haben wir es hier mit dem Sprachspiel zu tun. Eine direkte Information über den Unfall, die von der Hauptfigur des Witzes vermittelt wird, stellt einen gewissen außersprachlichen Kontext dar, d.h. den Verlauf und die Folgen des Unfalls. Die Überraschung wird aber im Schluss erzeugt, wenn die Phrase Zimmer dreizehn bis achtzehn an die schrecklichsten und unvorstellbaren Folgen des Unfalls erinnert, so dass der Rezipient keine Fragen mehr stellen braucht, um den Sinn des Witzes zu verstehen.

Ein unerwartetes Ende in einem Witz kann eben aus dem Kontext resultieren, wenn zum Schluss eine ganz andere als hervorgesehene Reaktion der Witzfigur dargestellt wird, z.B.

\section{(45) "Herr Müller", ruft die Nachbarin aufgeregt, "Ihre Frau ist so- eben im Dorfweiher ertrunken!" "Dann sollten Sie sofort die Seu- chenstelle verständigen!"}

Die Ehefrau-Figur wird von ihrem Mann als eine Seuchenquelle präsentiert, was aber nicht aus der einleitenden Aussage resultiert, denn es wird mit dem einführenden Satz eine traurige Nachricht vermittelt. In diesem Fall spielt das Außersprachliche die entscheidende Rolle, denn erst die zwischen den Zeilen ausgedrückte Meinung des Mannes über seine Frau macht das Komische aus.

Der Doppelsinn der Aussage entsteht auch dann, wenn bestimmte Gebrauchsvariante eines Lexems bzw. eine Wortgruppe eben zwei Interpretationen zulässt, z.B. im folgenden Witz wird mit der Wendung jdn nackt malen gespielt, was einerseits auf den Maler und andererseits auf das Modell bezogen werden kann.

\section{(46) Der Maler sagt nachdenklich zu seinem Modell: \\ "Ich überlege mir gerade, ob ich Sie nicht lieber nackt malen soll." \\ "Um Himmels willen”, entsetzt sich das Modell, "bleiben Sie bes- ser angezogen!"}

Interessant kann dabei aber auch eine andere Erscheinung sein, wenn das Komische ganz zufällig zustande gebracht wird, vor allem dann, wenn der Erzählende selbst eine oder andere Bedeutungsvariante des gebrauchten polysemen Wortes nicht kennt. Im unten zitierten Witz geht es um das Spiel mit der Wendung Selbstgespräche führen und dem Wort allein, was aber im folgenden Witz geschickt ausgenutzt wird, indem die Figur des Zeugen durch seine Antwort selbst das Ge- 
richt in eine humoristische Situation bringt, was aus dem falschen Gebrauch der genannten lexikalischen Elemente resultiert.

\section{(47) "Wissen Sie", wird der Zeuge gefragt, "ob der Angeklagte Selbstgespräche führt, wenn er allein ist?" "Ich war nie bei ihm, wenn er allein war" bedauert der Zeuge.}

Als nächstes Beispiel kann der folgende Witz genannt werden, in dem die Wendung ein Kind erwarten in einem doppeldeutigen Kontext erscheint, so dass es möglich ist, zwei verschiedene Interpretationen zuzulassen, d.h. die von 1. 'schwanger sein' und 2.'auf jdn warten'.

(48) Ein Vater will sein Kind von der Schule abholen. "Erwarten Sie ein Kind?" fragt eine Lehrerin "Nein, ich bin einfach so dick!" erwidert der Vater

Die beiden genannten Bedeutungen können verschiedene außersprachliche Kontexte zulassen, wobei in dem Witz 'schwanger sein' und 'dick sein' vom dem Vaterfigur gleichgesetzt werden. Demzufolge hat der auf der Polysemie basierende Witz den größten Umfang und tritt am häufigsten auf [Bystroń 1960; Buttler 1936].

\subsection{Fremdwort als Mittel des Humoristischen}

Die scherzhafte Kommunikation bedient sich auch der fremdsprachigen Elemente, die bewusst von den Autoren in die Texte eingesetzt werden. Es ist jetzt nicht meine Aufgabe, die Problematik der Fremdwörter im Deutschen und Polnischen ${ }^{44} \mathrm{zu}$ besprechen, sondern sie kurz zu skizzieren, um veranschaulichen zu können, welche Funktion sie in den humoristischen Texten haben können und aus welchen Gründen sie von den Autoren gewählt werden.

Die Globalisierung bewirkt das Aufkommen von Internationalismen. Auf der interlingualen Ebene sind sie Bestandteile eines ,, ausdrucksseitig nicht realisierbaren" Interlexems [Glück 1993, S. 277]. Als in verschiedenen Sprachen bekannte Lexeme haben sie eine ähnliche oder gleiche Formative und Bedeutungen

${ }^{44}$ Mit dieser Problematik setzte ich mich in meiner Dissertation auseinander: Sikorska [2001], und in meinen früheren Beiträgen zu den Problemen der entlehnten Lexik in der entlehnenden Sprache:[1997, 2002, 2004, 2008],; Sikorska-Bujnowicz [2011]. 
in allen Sprachen, z.B. engl. $\mathrm{Hi}-\mathrm{Fi}-\mathrm{dt}$. $\mathrm{Hi}-\mathrm{Fi}-$ poln. $\mathrm{Hi}-\mathrm{Fi}$, engl. Computer - dt. Computer - poln. komputer, engl. telephone - dt. Telefon - poln. telefon, engl. Fax - dt. Fax - poln. fax, engl. Radio - dt. Radio - poln. radio, engl. toilet - dt. Toilette - poln. toaleta usw. Oft stehen sie als Termini und gehen auf Latein oder Griechisch zurück, wie z.B. dt. Morphologie < Morph + griech. logos 'Lehre' poln. morfologia. Dies kann die Herausbildung der faux amis bewirken. Es sind morphologische und idiomatische Entsprechungen in zwei verschiedenen Sprachen, wenn zwei Lexeme scheinbar einander entsprechen, wobei sie unterschiedliche Referenzbereiche haben. Man denke u.a. an engl. carnation 'Nelke' vs. poln. karnacja 'Teint' bzw. dt. Fragment 'nicht fertiggestelltes Werk' vs. poln. fragment 'Teil des Ganzen' oder dt. bekommen vs. engl. to become 'werden'usw.

In den Sketchtexten werden oft vor allem Modewörter gebraucht, z.B. sowohl im Polnischen als auch im Deutschen sind es u. a. Wörter englischer (cool, bye, VIP, remake, topless, rock'n'roll), französischer (voila, vis-á-vis, á propos, faux-pas, foyer, adieu) oder italienischer Herkunft (ciao, bella) usw. Meist werden fremdsprachige Lexeme von einzelnen Interessengruppen entlehnt. Mit der Zeit werden sie feste Bestandteile der jeweiligen Berufssprachen, z.B. der Handwerkersprache: poln. hebel $<\mathrm{dt}$. Hobel, poln. muterka $<\mathrm{dt}$. Mutter (als 'Teil einer Schraube'), poln. waserwaga $<\mathrm{dt}$. Wasserwaage, poln. szuwaks $<\mathrm{dt}$. Schuhwachs, poln. kielnia $<$ dt. Kelle usw.). Diese Lexik erscheint dann auch im Rahmen der für die Zwecke des Humoristischen gebrauchten Handwerkersprache.

Als Fremdwort im engeren Sinne ist ein Wort fremder Herkunft zu verstehen, das seine ursprünglichen phonologischen und morphologischen Merkmale beibehalten hat. Es passt sich nicht ganz an die Regeln des entlehnenden Sprachsystems an. Seine ursprünglichen Merkmale werden beibehalten und sie betreffen die phonologische (Phonembestand, Phonemposition, Akzent) und/oder graphische (Graphembestand, Graphemkombination, Graphemposition) und/oder morphematische Ebene, z.B. dt. engl. barbecue - dt. Barbecue - poln. barbecue; engl. leasing - dt. Leasing - poln. leasing; eng. computer - dt. Computer - poln. komputer).

In Bezug auf Gebrauch, Verständlichkeit, Denotatsbezug und Verbreitung lassen sich die Fremdwörter in folgende Gruppen unterteilen [Glück (Hrsg.) 1993, S. 197; Bußmann (Hrsg.) 1990, S. 253]:

- allgemein bekannte und geläufige Internationalismen (engl. computer - dt. Computer - poln. komputer, engl. hotel - dt. Hotel - poln. hotel),

- Fachwörter (engl. laser - dt. Laser - poln. laser, engl. hardware - dt. Hardware - poln. hardware, engl. software - dt. Software - poln. software), 
- Bezeichnungsexotismen, die das nur für ein bestimmtes Land Charakteristische wiedergeben (dt. Tamtam - poln. tamtam - engl. tamtam, engl. igloo - dt. Igloo - poln. igloo, dt. Kreml - engl. Kreml-poln. Kreml),

- Modewörter (aus dem Englischen - dt./ poln. prima, super, cool)

- Salonwörter, die als Mittel zur Aufwertung eigener Position in der Gesellschaft gelten und als emotional stärker markierte Wörter neben den einheimischen Entsprechungen mit derselben Grundbedeutung, aber mit anderer Konnotation, stehen und von den Sprechern meist bevorzugt werden (aus dem Französischen, z.B. dt. Courage - poln. kuraż neben poln. odwaga, dt. Audienz - poln. audiencja neben poln. wizyta).

Die Fremdwörter werden auch deswegen gebraucht, weil sie Fachbegriffe aus dem Bereich der Wissenschaft (z.B. poln. Amper, Volt) und der Technik (z.B. poln. maszyna, rajzbret, klupa) sind bzw. weil sie im Unterschied zu stilistisch neutralen einheimischen Wortern einen markierten Charakter haben, z.B. dt. Visage: Gesicht, poln. tysina 'Glatze': glaca, oder aber weil sie das lokale, historische oder nationale Kolorit zum Ausdruck bringen (z.B. dt. Baklava - poln. baklawa, dt. Halva - poln. chatwa, dt. Sushi-poln. sushi).

Innerhalb der humoristischen Texte wird vor allem auf die fehlenden Sprachkenntnisse der Witzfiguren angespielt, was zusammen mit dem situativen Kontext eine interessante Mischung mit viel Biss, aber vor allem mit viel Humor ausmachen kann. In „Was guckst du?“ sind es diese Fälle, wenn das Deutsche, das Arabische, das Türkische, das Englische oder aber auch das Polnische als Fremdsprachen und somit als Quellen aller möglichen Fehler gesehen werden. Bei „Paranienormalni“ sind es die fehlenden Kenntnisse des Polnischen und des Englischen. Die Paradebeispiele für diese Art Witze werden des Weiteren detailliert präsentiert und besprochen. 


\section{SPRACHLICHE MITTEL DES HUMORISTISCHEN IN ,WAS GUCKST DU?“6}

\subsection{Struktur und Sprache von „Was guckst du? als Ethno-Comedy}

Im deutschen Fernsehen hat die Comedy-Sendung „Was guckst du?“ eine besonders wichtige Rolle zugeschrieben bekommen, was vor allem auf ihren multikulturellen Charakter zurückzuführen ist [Sikorska 2009a]. Zur Quelle des Humoristischen wird das Leben eines in Deutschland lebenden Einwanderers, was nicht nur mit den Problemen mit der deutschen Sprache zusammenhängt, sondern auch mit den Schwierigkeiten, die mit dem Alltag verbunden sind. Der Moderator - Kaya Yanar, der sich seiner türkisch-arabischen Abstammung rühmt und immer betont, wie wichtig für ihn die Ausländerproblematik bei der Erstellung der witzigen Texte ist. In seiner Show wird geschickt mit den Stereotypen gespielt. Zur Zielscheibe werden in diesem Fall die ausgedachten und meisterhaft dargestellten Figuren der in Deutschland lebenden Ausländer - Türken, Polen, Italiener, Russen, Inder oder Araber. Sie sind sympathisch und haben viele Fans. Wichtig ist, dass sich die Sketche in der deutschen Szenerie abspielen, was als Mittel der Konfrontation verschiedener Kulturen dient.

Die von mir analysierte Sendung hat ein konkretes Format. Es ist kein einfaches Zusammenstellen von verschiedenen Sketchen, sondern ein abwechslungsreiches Angebot von witzigen Szenen und Geschichten, deren Autoren sich u.a. nach dem Kalender richten und auch diese Feste in Deutschland darstellen, die für die Ausländer ein wenig problematisch sein können, was auf den multikulturellen Charakter der deutschen Gesellschaft zurückgeht. Es kann an dieser Stelle eine Folge genannt werden, die ganz dem Weihnachten in Deutschland gewidmet ist. In der Sendung wird dann ein langer narrativer Witz über die Vorbereitungen auf dieses Fest präsentiert, in dem die in Deutschland herrschende Atmosphäre der Vorweihnachtszeit ausgelacht wird - Weihnachtsmärkte mit allen mit ihnen ver- 
bundenen Spezialitäten, wie z.B. Glühwein, das Warten auf den Weihnachtsmann, das Schmücken des Weihnachtbaumes usw.

\section{(49) Advents- und Weihnachtszeit in Deutschland \\ Am Sonntag ist schon ja erster Advent. Da rücken die Menschen wieder ein bisschen näher zusammen. Vor allem im Kaufhaus.}

Der Witz wird unterbrochen und ein kurzer Satz formuliert, der mit Gesten und Mimik unterstützt wird. Die Stimme von Yanar ist ganz anders, sie erinnert an eine weibliche Stimme, Yanar versucht mit diesem Satz eine Szene im Kaufhaus nachzuahmen, wenn die Deutschen im Einkaufsrausch sind und alles Mögliche kaufen wollen.

"Können Sie bitte die Kerze aus meiner Nase nehmen???!!"

Yanar erinnert auch an seine Familie, die Probleme damit hatte, sich an die neue Tradition zu gewöhnen.

Für meine Familie waren diese Weihnachtvorbereitungen ja neu, als wir nach Deutschland kamen. Aber wir haben alles mal getestet - Glühwein, Zimtsterne oder auch Marzipankartoffeln. Hier macht er eine kurze Pause, damit auf diese Weise noch Spannung steigen kann. Das war mein erster und letzter Kartoffelsalat! Er lächelt, das Lächeln ist echt, was wir an den Augen erkennen können, die mitlächeln. Anfangs haben wir sogar den ersten Advent mit dem ersten April verwechselt. Das machte uns dann Spaß - Scheiße in jeder Plätzchendose. Advent, Advent! Die Stimme lässt uns erkennen, wie glücklich er ist. Ich hab etwas anderes in der Dose... Schei... Beee!!! Jetzt erkennt man an seinem Gesichtsausdruck vor allem Überraschung und Wut.

Im Zusammenhang mit den Weihnachtsvorbereitungen erwähnt Yanar auch, was die Ausländer mit dieser Zeit assoziieren

Mittlerweile sind wir Ausländer aber auch voll auf Weihnachten eingestellt. Türken legen ihren Schlenker tiefer, im Verkauf statt Rosen - Tannenbäume, und Chinesen suchen sich ein Tier für ihren Weihnachtsbraten aus. 
Hakan steht total auf diese Adventskränze mit Äpfeln und Walnüssen, obwohl er Walnüsse immer falsch versteht. "Was? Das sind Walnüsse? Du armer Fisch!"

Wieder fällt auf, dass in die erzählte Geschichte andere witzige Bemerkungen eingebettet sind, wo schon mit der Sprache gespielt wird, wie hier, wenn unter Walnüssen Hoden verstanden werden, also die umgangssprachliche übertragene Bedeutung tritt in diesem Kontext in den Vordergrund.

Es wird außerdem noch den Stereotypen nachgegangen, indem Süleyman, ein türkischer Radiomoderator, als Dieb dargestellt wird, für den der Adventskalender etwas anderes ist als für die Kinder, die hinter jeder Tür dieses Kalenders eine Schokolade finden können.

Mein Kumpel Süleyman freut sich auf die Adventszeit. Der macht jede Nacht ein Türchen auf. Kaya macht ein Miene, die eine Überraschung ausdrückt, zieht die Augenbrauen hoch. "Oh, ein Rembrandt. Danke, Christkind!" Er macht Süleyman nach, lächelt zufrieden und glücklich.

Die Weihnachtsmärkte in Deutschland sind in diesem längeren Witz zum Thema und zur Zielscheibe geworden, indem Kaya sie detailliert beschreibt und unter anderem auf die Schwächen der Deutschen zeigt

Besonders liebe ich am Advent die Weihnachtsmärkte. Da kann man sich von seiner Nase leiten lassen. Wo es nach [...] Zimt riecht, gibt es Plätzchen und wo es nach Kotze riecht - den Glühwein! Ja, ein Glühweinstand. Glühwein. Am Glühweinstand merkt man immer - Besinnlichkeit und Besinnungslosigkeit liegen dicht beieinander.

In diesem Falle wird schon wieder mit der Sprache gespielt, indem dem Verb liegen in der Verbindung mit dem Adverb dicht in dem genannten situativen Kontext die Bedeutung von 'nach zu viel Alkohol ohne Bewegung liegen ' zugeschrieben wird, was auf den Zustand der Glühweinkonsumenten zurückzuführen ist, genauso wie das Zusammenstellen von Besinnlichkeit und Besinnungslosigkeit.

Interessant ist auch die Tatsache, dass in diesem narrativen Witz selten Fehler auftauchen, diese erscheinen erst in den stilisierten kurzen Präsentationen, die mit konkreten Szenen verbunden sind. Als komisch sind eben die Ereignisse selbst einzustufen, die aus der Konfrontation mit einer neuen Mentalität und einer 
neuen, bisher fremden Kultur resultieren. Dabei werden andere Sachen mitberücksichtigt, d.h. die Assoziationen mit den Aktionen zugunsten der Ausländer in Deutschland

K: Wir Ausländer finden den Advent immer toll. Zumindest anfangs. Endlich wieder Lichterketten. Hier macht er eine Pause, als ob er sich etwas überlegen würde. Sein Gesicht zeigt etwas Enttäuschung. Ach so, wegen Weihnachten und nicht wegen uns...

In diesem Abschnitt wird nicht die wortwörtliche Bedeutung von Lichterkette als Christbaumschmuck verstanden, es geht hier um eine Aktion, während deren man die Lichter als Symbol der Solidarität mit den Ausländern anzündet.

Es wird auch ein weiteres Zeichen von Weihnachten mitberücksichtigt der Weihnachtsmann und bei der Gelegenheit auch die Briefe, die man an ihn schreibt. Die Fragen danach, wie man einen Brief an den Weihnachtsmann richtig formulieren sollte, sind in dem folgenden Abschnitt zur Zielscheibe geworden. Die deutschen Anreden und typische Amtssprache werden ausgelacht.

K: Der Advent ist natürlich auch die Zeit der Wunschzettel. Dabei geht es natürlich um die richtige Anrede. "Lieber Weihnachtsmann!..", nee, "Lieber Herr Weihnachtsmann!" nee, "Lieber sehr geehrter Herr Weihnachtsmann!" Scheiße!!! Oder... oder... die türkische Anrede "Alde! oder "Sehr geehrter Herr Alde!"

Des Weiteren wird ein deutscher Versandkatalog des Sexshops Beate Uhse angesprochen als Katalog, der auch die Wünsche der Väter erfüllen kann.

K: Bei meinem Vater hieß das nicht Weihnachtszettel, sondern Beate-Uhse-Katalog. Schickt mir dies und dies.

Zum Schluss erwähnt er noch vielleicht die wichtigste Sache an einem Weihnachtsgeschenk - den Kassenbon, den man aufbewahren soll. Ausgelacht wird dabei noch die Tatsache, dass sich die Deutschen auf die Ergebnisse verschiedener Tests verlassen mögen, bevor sie sich zum Kauf von etwas entscheiden.

$\mathrm{K}$ : Aber denken Sie dran, wichtig ist nicht, was man schenkt oder welche Marke oder wie teuer. Wichtig ist, dass man den Kassenbon 
aufbewahrt und wenn Sie dann mal einkaufen, sollen Sie sich besser nicht von irgendwelchen Meinungsforschern aufhalten lassen.

Für die Zwecke der Sendung greifen die Autoren auch zu den Originaltexten, die sie als Quelle des Humoristischen gebrauchen. Im Laufe der Sendung wird noch ein weiteres Problem angesprochen - die kaum verständliche Bedienungsanleitung einer Lichterkette, wo es von den Fehlern wimmelt. Es sind grammatische Fehler (50a-c), die Fehler in der Syntax (fehlendes Subjekt, fehlendes Objekt im Satz (50a), der Gebrauch von man anstelle von es (50c):

(50) „Achtung! (50a)Wenn die Lampe nicht wäre, darf man nur mit der Ersatzlampe oder gleichen Typen Lampe wechseln. Bei dem Wechseln muss die Lampe nach gleicher Richtung in die Lampedose (50b) zustecken sein. Wenn man die Lampe nicht wechseln könnte, kann man zum Beispiel (50c) man mit dem Hersteller abreden $[\ldots]^{\prime \prime}$

Auf diese Weise ist auch die Intertextualität mit im Spiel, denn ein Originaltext wird als Beispiel der unverständlich geschriebenen Bedienungsanweisungen genannt, die nicht nur dann schwer zu verstehen sind, wenn der Originaltext nicht ganz korrekt übersetzt wurde, sondern allgemein - wegen der zu komplizierten Fachsprache, die dort auftaucht. Diese Anleitung wird von Yanar noch kommentiert.

$\mathrm{K}$ : Da fragt man sich, wie es zu solcher Übersetzung überhaupt kommen kann. Wir haben einen deutschen Übersetzer gefunden, der zu diesem Thema interessante Insider-Informationen liefern kann. Natülich will er aus Angst vor seinen Kollegen unerkannt bleiben, deswegen befindet er sich in einem abgedunkelten Raum.

- Guten Abend!

- Guten Abend, Herr Yanar!

- Sie behaupten also an der solchen Übersetzung steckt die regelrechte Übersetzungsmafia?

- Nun, es ist folgendermaßen, dass...

- Hallo, ich muss es kurz sauber machen...

- Ja, danke für Informationen. Übrigens [...], die Lichterkette und viele andere Preise können Sie also beim Adventskalender gewinnen unter www.wasguckstdu.de [...] 
Die Autoren lachen auf diese Weise die Übersetzer aus, die ihren Kollegen alles Schlimme anhängen wollen, weil diese ihnen die Aufträge wegnehmen. In dem zitierten Abschnitt erscheint schon die Verweisung auf die Internetseite von „Was guckst du?", so dass somit auch die Funktion der Sekundärtexte um die Sendung herum betont wird.

Wie in vielen Folgen tauchen auch in dieser die Parodien der Werbung - in diesem Fall geht es um „Miracolli - die beste Nudel der Welt“, die im Programm als „Irakoli, die beste Nudel der Welt“ erscheint. Es geht also um die Anspielung auf den Irak und auf Hussain. Wichtig dabei sind alle außersprachlichen Elemente - irakische Soldaten machen Werbung für irakische Pasta, zum Schluss wird sogar geschossen. Das Erscheinungsbild der Soldaten ist hier von Bedeutung - Yanar sieht wie Hussain aus, verzerrt das Gesicht, das Essen von „Irakoli“ sieht gar nicht vorteilhaft aus, das Essverhalten der Soldaten erinnert sogar an Tiere.

Im Laufe der Zeit haben die Autoren auch daran gedacht, die einzelnen Sketche in Form einer Serie zu präsentieren. Dazu gehören u.a.

- Geschichte von Familie Yildirim „Nicht mit meiner Tochter“ (somit wird der Film „Nicht ohne meine Tochter“ angesprochen),

- Fahrschule von Kelal Yildirim mit dem einzigen deutschen Schüler Manfred Winter, der von dem Fahrlehrer ständig veräppelt und sogar schikaniert wird, was nicht nur das Spiel mit den Assoziationen mit seinem Namen bedeutet (Manfred Winter heißt dann Weihnachtsmann, Schneemann, Rutschpartie),

- Geschichte von dem sympathischen Inder Ranjid, der unterwegs mit seiner Kuh ist, Deutschland kennen lernt, verschiedene Berufe auszuüben versucht und dabei ganz gute Kenntnisse im Computerbereich und dem Tantrasex hat,

- Geschichten von Hakan, dem türkischen Türsteher,

- „Tagesguck“ mit Hakan als Moderator

- Geschichten mit der russischen Helleseherin Olga

- Interviews von Tarek ab del Kalek

- Liebestreffen von dem machohaften Italiener Francesco

- Geschichten mit dem Sirtaki - Mann, der immer bereit ist, allen zu helfen

- Geschichten mit Süleyman, der mit seinem Süleyhund eine besondere Art Radiosendung führt

Alle für die Zwecke der Sendung ausgedachten Sketche kreisen um die Thematik des Ausländerlebens in Deutschland. Sie werden noch um die Präsentationen der Ausschnitte aus den Programmen aus aller Welt, vor allem aus den arabischen Ländern, ergänzt. Dabei werden lustige Bilder gezeigt, wie z.B. eine aus dem Becher ihr Getränk schlürfende alte Nonne mit einem Auge, ein lachender 
Mann mit einem Zahn, arabische Musiker in einem Fernsehstudio, in dem die Dekoration zusammenfällt und vieles mehr. Es wird auch die polnische Synchronisation der ausländischen Filme ausgelacht, weil es nur eine Person ist, die diese Dialoge vorliest, am meisten ist es ein Mann, dessen Stimme zu den Frauengestalten gar nicht passt, was am Beispiel von der Synchronisation des Films „Alf“ gezeigt wird. Yanar wiederholt sogar die Phrase Ani twój, ani mój und betont auch, dass die polnische Version dem Tempo nach nicht dem Original entspricht. Yanar sucht sich interessante Fälle aus und stellt sie im Programm dar. Das häufigste Wort, das als Homophon zu betrachten wäre, ist arabisch Aschrat 'zehn', das als Arschratte wiedergegeben wird und das Lachen des Publikums im Studio hervorruft.

Der schon erwähnte Hakan wird in verschiedenen Situationen platziert - bei der Arbeit als Türsteher, wenn er seinen Satz Ey du, du kommst hier nicht rein! wiederholt, zu Hause, wenn der Gerichtsvollzieher ihn besucht, in der Werbung für einen Dreier - BMW, im Nachrichtenprogramm ,tagesguck“, das an das Original „Tagesschau“ erinnert und schließlich auch als werdender Vater, der seiner Freundin bei der Geburtsvorbereitung helfen soll, dargestellt wird.

\section{(51) Hakan und Geburtsvorbereitung}

Im Studio von Yanar sitzen Aische und Hakan. Die Frau versucht Hakan dazu zu überreden, dass er bei der Geburtsvorbereitung dabei ist.

A: Hakan, gehst du jetzt zu der Geburtsvorbereitung oder ne, he? $H:$ (51a) Hast du Baby in den Ohren? Ich habe gesagt, ich muss Kayas Mütze finden.

A: Aaa!!!

H: (51aa) Mach dir Knack am Arsch oder was?

A: Du Idiot, es ist das Baby, das Baby kommt. Könntest du mir helfen?

$H$ : (51b) A, ruf mal an den Doktor Stefan Frank oder was! ScheiBe!

A: Der Stuhl, der Stuhl, der Stuhl!!!

$\mathrm{H}: \mathrm{Ja}, \mathrm{ja}, \mathrm{ja}$, der Stuhl, eine gute Idee mit dem Stuhl!

$A: \mathrm{Ja}, \mathrm{ja}$, der Stuhl!

$H$ : Und du?

A: Ey, (51c) wie schon die Wehen, doch muss ich entspannen, o.k.?

Du musst mich jetzt massieren. Tu dies für Baby!

$H$ : Da komm ich, komm jetzt dran..

A: Muss ich jetzt versuchen, das wegzuatmen.

$H$ : Was? Das Baby? 
A: (51d) Den Schmerzen, du Idiot! Machst du mit?

$H:$ Ah, So...

A: Eee, mach du richtig!

$H:$ Korrekt?

A: (51e) Gehst du mal runte auf den Boden? (51f) Haltest du Hände auf den Knien

H: Scheiße!

A: Genau, jetz† mach mich den Becken locker..

$H$ : Das soll gut sein für das Baby?

A: Keine Ahnung, aber Mama macht Spaß!

$\mathrm{H}$ : Kommt das Baby jetzt raus oder nicht?

A: Neee, (51g) aber hast du wenigstens gemacht Geburtsvorbereitung!

$H:$ E! Pass du auf! Dich verarsche ich auch, wenn ich schwanger bin oder so!

Im genannten Sketch fällt das Türkisch-Deutsch auf. Es werden mit Absicht fehlerhafte Formen gebraucht, wie (51c) *wie schon die Wehen, (51e) *Gehst du mal runte auf den Boden?, (51d) *Den Schmerzen und (51f) *Haltest du Hände auf den Knien und falsche Wortfolge erscheint im Satz (51g) *aber hast du wenigstens gemacht Geburtsvorbereitung! Es wird auch auf einen bekannten deutschen Serienfilm „Dr. Stefan Frank“ verwiesen - (51b) A, ruf mal an den Doktor Stefan Frank oder was! und ein Element der Umgangssprache gebraucht (51a)Mach dir Knack am Arsch?oder aber eine Wendung (51aa) Hast du Baby in den Ohren?, wo statt des Lexems Watte (wie in der Wendung Watte in den Ohren haben im Sinne von 'nicht reagieren') das Wort Baby erscheint.

Die Ausländerproblematik betrifft die ganze Sendung, es können aber konkrete Sketche genannt werden, in denen es um das Leben der Ausländer in Deutschland geht, z.B.

\section{(52) Umfrage auf der Straße}

D: Entschuldigung, wir machen eine Unterschriftenaktion zum Thema "Ausländer". Was sagen Sie zu den Ausländern?

Der angehaltene Türke will sich aber nicht dazu äußern, sondern schnell weiter gehen.

T: Bitte, (52a) müsse nach Hause gehen! 
Der Deutsche ist mit der Antwort zufrieden, obwohl der Befragte etwas Anderes gemeint hat und lächelt.

D: Genau, müssen nach Hause gehen. Bitte unterschreiben Sie! Danke schön! Günther...

In der genannten Szene werden die gegen Ausländer in Deutschland protestierenden Deutschen dargestellt, die die fehlende Deutschkenntnis bei den Ausländern für ihre Zwecke ausnutzen. Die fehlerhafte, pseudohomophone Form (52a)*müsse nach Hause gehen bildet demzufolge den Kern des Humoristischen, um den herum die Pointe entsteht.

In einer anderen Szene wird ein Spot gegen Ausländerfeindlichkeit gedreht. In diesem Spot spielen Neonazis und Kaya. Die Neonazis haben die Baseballschläger, schlagen auf Kaya zu, wobei die Szene mehrmals wiederholt werden muss, weil der Regisseur nicht ganz zufrieden ist. Wichtig dabei ist aber die Tatsache, dass die Neonazis echt zu sein scheinen, weil sie den armen Ausländer tatsächlich verprügeln, so dass alles im Endeffekt realistisch wirken kann. Die Szene endet mit dem Spruch „Die Ausländerfeindlichkeit ist Scheiße!“, womit gezeigt werden kann, wie die Ausländer in Deutschland behandelt werden, sogar wenn es nur um den Spot für einen guten Zweck geht.

Die Frage nach den Deutschkenntnissen ist das Motiv von vielen Szenen in „Was guckst du?“', es werden verschiedene Situationen dargestellt, wo diese dann die entscheidende Rolle spielen, z.B.

\section{(53) Türken und Polizeikontrolle}

Ein Auto wird von der Polizei angehalten. Am Steuer sitzt ein Türke, neben ihm - seine Frau. Der Polizist fragt den Fahrer nach dem Führerschein.

P: Ihre Dokumente bitte.

T: E? Der Türke scheint die Frage nicht zu verstehen.

$P:(53 a) \mathrm{Na}$, Sie wissen schon...

T: (53b) Nazis? Nazis?

P: Dokumente: Ausweis, (53c)Führerschein...

$\mathrm{T}$ : (53d) Führer, Führer, Adolf Hitler! Hilfe!

P: Schon gut, schon gut, fahren Sie weiter! Der Polizist gibt auf und lässt sie los. Es stellt sich heraus, dass der Türke keinen Führerschein hat und seine mangelnden Deutschkenntnisse nur vortäuschte. 
In dieser Szene fällt vor allem das Spiel mit den homophonen Formen auf (53a) Na Sie wissen vs. (53b) Nazis und (53c) Führerschein, wenn von dem ganzen Determinativkompositum nur das Bestimmungswort (53d) Führer gebraucht wird und auf Hitler bezogen wird. Es wird außerdem noch auf eine weitere Erscheinung aufmerksam gemacht - die Probleme im Alltag können sehr schnell gelöst werden, wenn ein Ausländer alles auf die Schwierigkeiten mit der Sprache zurückführt und sich auf diese Art und Weise zu entschuldigen versucht.

Interessant ist dabei auch, dass man sich über die Deutschen lustig macht, indem man die Szene wie „Ausländer raus!“ zeigt, wenn es vor dem Haus eines Ausländers eine Demonstration gibt und nach den Ausrufen „Ausländer raus!“ aus dem Haus eine Gruppe Ausländer rauskommt. Es wird demzufolge mit der Doppeldeutigkeit gespielt, indem das Wort raus wortwörtlich und nicht als Aufruf dazu, Deutschland zu verlassen, verstanden wird.

Der sympathische Inder Ranjid versucht sich auch in der Arbeit bei „Was guckst du?" und greift sogar nach der Mütze, dem Markenzeichen von Yanar, die er als Putzlappen benutzt und zum Schluss doch erkennt, womit er geputzt hat

\section{(54) Ranjid und Kayas Mütze}

$\mathrm{R}: \mathrm{Na}$, Kaya, jetzt kriegst du ein Geschenk.

$K:$ Ey! Was hast du in der Hand?

R: (54a) Meine neue Putzlappe, habe ich es (54b) in der Dusche gefunden, sehr (54c) strapaziert. Wirklich! Der geht einfach nicht kaputt, der hat mehr Leben und wir haben schon 100! Gleich nur mal 99.

K: $O$, toll! Meine Mütze, gib her!

R: (54d) Du willst lieber nicht aufziehen...

$K$ : Findest du? Ich wollte schon immer mal ohne Mütze...

R: Setz die auf!

K: Schon gut, schon gut!

Wieder fällt es auf, dass fehlerhafte Formen zum Zwecke des Humoristischen eingesetzt werden. Es sind hier: falscher Artikel (54a)*meine nеие Putzlappe, falsche Präposition (54b)*in der Dusche statt unter der Dusche und fehlendes Objekt im Satz (54d)*Du willst lieber nicht aufziehen und der Gebrauch von (54c) strapaziert statt strapazierfähig.

Ranjid lernt die Putzfrauen, die die Fernsehstudios putzen, kennen und sie machen ihn mit seinen Pflichten bekannt. Im Gespräch erscheinen die Marken der Putzmittel Meister Proper und General (Fresh) und somit wird der intertextuelle Charakter dieses witzigen Textes sichtbar. 
(55) Ranjid im SAT.1-Studio

R: Hallo!

P: Hallo!

R: Kurz bevor wir anfangen - wer von euch ist Meister Proper und wer ist hier der General?

P: Ich bin General und jetzt geht's los!

Ranjid geht von Tür zu Tür und nennt die bekannten Namen auf den Schildern R: Das ist Harald Schmidt, das ist Anke Angelke und der Ingolf Lück und die Benita.

Er fängt an zu putzen, gebraucht dabei die ihm zugeteilten Putzmittel.

R: Eins, zwei, drei und so... eins, zwei... ich habe es gleich benutzt und ein bisschen geputzt. So000... habe ich jetzt die Mattscheibe geputzt, jetzt ist es sauber.

P: Gut!

R: Es ist jetz† Klarscheibe!

Ranjid spielt jetzt in einer Quizshow und versucht auf die letzte Frage zu antworten.

P: Und jetzt die Meisterfrage - es geht um 16000 Mark. Passen Sie gut auf! Womit reinigt man am schnellsten? Erstens: mit Glasreiniger, zweitens - mit Spiritus, drittens - mit Spucke.

R: Mit Spucke?

P: Richtig! 16000 Mark!

Das Format von „Wer wird Millionär?“ wird im Rahmen der Sendung oft ausgenutzt, das beste Beispiel dafür ist der Sketch „Deutscher Integrationstest“ .

\section{(56) Deutscher Integrationstest}

Szene in einem deutschen Büro. Ein Türke will den deutschen Pass bekommen und muss die drei letzten Fragen des Integrationstestes beantworten.

Beamter: Ja, Herr Egötürk, nur noch drei Fragen und Sie haben den deutschen

Integrationstest bestanden. Im Hintergrund läuft die Musik aus ,Wer wird Millionär? "Welche Unterhose trug Helmut Schön bei der WM 1954 in der Schweiz beim Endspiel Deutschland - Ungarn, das wir mit 3:2 gewonnen haben?

Kandidat: Das ist eine Fangfrage. Erstens Trainer war (56a)nichts Helmut Schön, sondern Sepp Herberger und zweitens (56b) Unterhosenfarbe war blau. 
B: Gut, gut, richtig. Im Hintergrund läuft die Musik aus ,, Wer wird Millionär?".

Aber wir haben noch zwei Fragen. Er guckt den Kandidaten ironisch und bösean. Was sagte August Heinrich Hoffmann von Fallersleben, der uns unsere Nationalhymne schenkte, am 26. August 1841 auf Helgoland um genau 19.45?

K: Das kann ich Ihnen genau sagen. Genau um 19.45 sagte er: "Wo habe ich diesen Liedtext hingetan?" Bei der Antwort gestikuliert er heftig.

B: Gut, gut, schöööön... Aber jetzt zur entscheidenden Frage. Dann können Sie Ihren deutschen Pass mit nach Hause nehmen. Was geschah in Deutschland von 1933 bis 1945?

K: Zwischen' 33 und ' 45?

B: Der Beamte nickt.

$\mathrm{K}: A$, ich weiß, ich weiß. Er wird traurig, zuckt mit den Augenbrauen, verzieht das Gesicht zur Grimasse und überlegt sich die Antwort. Doch, doch, ich weiß!

B: Ja??????? Jetzt erkennt man an der Mimik, dass der Beamte völlig überrascht ist. Aber er hofft zugleich darauf, dass der Kandidat durchfällt.

$\mathrm{K}$ : Ich kann mich einfach nicht erinnern... Er ist sehr traurig und fängt an zu weinen, da er weiß, dass er die Chance verpasst hat.

B: Bravo!!!!!!! Ich auch nicht! Er macht die Begrüßungsgeste der Nazis. Herzlich willkommen in Deutschland! Sehr glücklich überreicht er dem Türken seinen erträumten deutschen Pass.

Die Antworten des sich um den deutschen Pass bewerbenden Türken sind sowohl inhaltlich als auch sprachlich korrekt, einzige Fehler betreffen den falschen Gebrauch des Negationswortes (56a) nichts, den fehlenden Artikel in der Konstruktion (56b) Unterhosenfarbe war blau. und die Aussprache. Das Gesagte wird einerseits mit der Mimik der beiden Gesprächspartner und andererseits mit der Musik aus der Sendung „Wer wird Millionär?“ unterstützt. Zum Thema der lustigen Texte dieser Art werden verschiedene Situationen, die das Leben eines Ausländers in Deutschland erschweren, nicht nur die Probleme mit der deutschen Sprache. Es wird u.a. der erwähnte Integrationstest angesprochen und der damit verbundene Traum vom deutschen Pass im Zerrspiegel präsentiert. Eine besondere Funktion hat in diesem Falle die Hintergrundmusik aus „Wer wird Millionär?“, die den Kontext um emotionale Elemente ergänzt, weil auf diese Weise alle Prozeduren und Schwierigkeiten beim Erlangen des deutschen Passes ironisch dargestellt werden können. Das hängt mit dem intertextuellen Charakter der Witze und der ganzen Sendung zusammen. Dieser kann aber nur dann zur Geltung kommen, 
wenn der Rezipient das Vorwissen aus allen damit zusammenhängenden Bereichen hat, das ihm das Verstehen der jeweiligen Kontexte nicht nur erleichtert, sondern es sogar manchmal ermöglicht.

Die ausgedachten Sketche werden von den Autoren nicht nur um die Ausschnitte aus den ausländischen Programmen ergänzt, sondern auch um die narrativen Witze und die wahren Geschichten, die Yanar erzählt, z.B.

(57) K: Guckst du "Was guckst du?", ich freue mich sehr, dass "Was guckst du?" Fans in ganz Deutschland hat, z.B. in Kuxhafen, guckst du hier! Da habe ich Folgendes entdeckt Yanar zeigt das Kennzeichenschild cux-Du 33 und unter den Kleinzuschauern wachsen sogar meine Nachfolger Kaya zeigt die von den Zuschauern geschickten Bilder, auf denen u.a. Kinder mit einer schwarzen Baskenmütze zu sehen sind und ihn nachahmen.

Der erwähnte kurze Abschnitt zeugt davon, wie wichtig es für die Autoren ist, den Kontakt mit den Zuschauern aufrechtzuerhalten, so dass die Sendung auch außer dem Fernsehstudio weiter lebt. Dank der Internetseite bleiben sie mit ihnen im Kontakt. Alle Sekundärtexte, die dort zu lesen sind, auch die Interviews, die Yanar gibt, haben einen großen Einfluss darauf, dass die Sendung nicht in Vergessenheit gerät und somit diese Art Humor, die Yanar anbietet, weiter gezeigt werden kann.

Yanar schlüpft auch in die Rolle von Süleyman, der im Radio zusammen mit seinem Hund Süleyhund auftritt und auf die Fragen und Bitten seiner Zuhörer antwortet. Die Gespräche sind dabei voll von Doppeldeutigem und Anspielungen auf Männer-Frauen-Relationen, z.B.

\section{(58) Radio Süleyman}

S: Hallo, Leute! Hier ist wieder Sprechradio Süleyman. Natürlich (58a) mit meine beste Freund Süleyhund. Scheiße! Muss mal wieder Gassi gehen. Braucht Auslauf. Also mach schnell. Erster Anrufer A1: Hallo. Süleymann! Ich bin traurig.

S: Traurig? Es ist ein Scheißname! (58b) Aber was geht, traurig? A1: Nein, du verstehst mich nicht! Ich heiße Thomas und bin traurig. Ich lerne einfach keine Frau kennen, weil ich immer Angst habe, dass sie nur mein Geld wollen, ich bin nämlich sehr reich.

S: Keine Angst, wenn du erst eine Frau hast, bist du bald nicht mehr reich...!

A1: Ich will keine Frau, die nur auf Materielles aus ist. Ich habe sogar schon einmal überlegt, mein Geld einfach wegzuschmeißen. 
S: Was??? Mach keine Dummheiten, (58c) helf ich dir, bist du alles los - Probleme und Geld. Scheiße!! Süleyman ist aufgeregt, weil sich ein anderer Zuhörer dazwischen meldet und unterbricht das Gespräch mit Thomas. Des Weiteren unterhält sich Süleymann u.a. mit Wachtmeisterin Hümmer.

A3: Guten Tag, hier spricht die Wachtmeisterin Hümmer. Uns liegt eine Anzeige wegen Umweltverschmutzung vor.

S: Umweltverschmutzung? Ich bin doch kein Umweltverschmutzer. (58d) Umweltverschmutzer ist Mann, der Sache wegwirft oder... Moment! Er kommt auf die Idee und verbindet sich wieder mit Thomas.

S: Traurig? Ich hab eine gute Idee für dich. Wirfst du doch Geld weg, packst du alles in großen Sack, wirfst du (58e) rückwärts von Rheinbrücke...

A1: Was??? Von mir aus... Und dann lerne ich eine Frau kennen?

S: Ja, (58f) vertraust du mir! Wieder verbindet er sich mit der Polizistin. Wachtmeisterin. (58g) Habe ich gehört, steht Mann am Rheinbrücke und wirft Müllsack in Fluss. Das ist ein Umweltverschmutzer, darfst du ihn festnehmen, mich nicht!

Süleyman spricht wieder mit Thomas und arrangiert ein Treffen mit der Polizistin.

S: Thomas, (58h) verspreche ich dich, wenn du den Sack geworfen hast, kommt nette Frau auf dich zu und nimmt dich mit, auch ohne Geld!

A1: Ohne? Danke, Süleyman! [...]

Die Szenen aus dem Radio „Süleyman“ gehören zu diesen, die als Teile einer Serie zu verstehen sind. Der Zuschauer kann sich an die Figur gewöhnen, sie entweder akzeptieren oder aber auch ablehnen. Das Spiel mit der Mimik und Gestik wird mit dem Sprachspiel noch verstärkt, so dass damit das Humoristische erzeugt werden kann, ohne dass man sich das merken kann, weil die dabei gebrauchte Lexik nicht lustig ist und weil erst die Mischung des Sprachlichen und des Außersprachlichen das Komische ausmacht. In dem genannten Sketch fallen vor allem fehlerhafte Formen auf - von den entweder grammatisch ganz unkorrekten Formulierungen (58a) *mit meine beste Freund Süleyhund, (58d) Umweltverschmutzer ist Mann, der Sache wegwirft oder..., (58f) vertraust du mir!, (58g)* verspreche ich dich, über teilweise falsche Elemente, z.B. (58e) rückwärts von Rheinbrücke, wo nur ein Artikel fehlt bzw. mehrere Artikelformen (58g) Habe ich gehört, steht Mann am Rheinbrücke und wirft Müllsack in Fluss. bis zu dem Konditionalsatz, der zusätzlich zur Verstärkung des stereotypen Bildes eines Türken beiträgt, nämlich (58c) helf ich dir, bist du alles los - Probleme und Geld. 
Die Autoren der Sendung haben auch ein gewisses Format ausgedacht, das als Garant für Witz und Spaß gilt - die Passantenbefragung. Es werden dabei verschiedene Sachen angesprochen - von den einfachen sprachlichen Problemen, über Fragen in Bezug auf die deutsche Kultur bis zu den ämtlichen Angelegenheiten.

\section{(59) Passantenbefragung}

K: Entschuldigen Sie, der Herr, die Dame da, haben Sie vielleicht eine Minute Zeit für die Umfrage für eine türkische Zeitschrift? Eine Frage: warum, glauben Sie, vertragen die Deutschen mehr Bier als Ausländer? Zieht die Augenbrauen hoch und wartet auf die Antwort.

P1: Das ist regelmäßig, trinken!

K: Was gefällt Ihnen bei Deutschen am wenigsten? Mit drei Worten müssen Sie sagen. Also, eine..., ja?

P2: Sie sind pingelig ${ }^{45}$

P3: Pingelig. Aber auch penibel ${ }^{46}$, manchmal

P2: Ja, penibel, ja..und...

$\mathrm{K}$ : Was heißt penibel eigentlich? Weil ich kenne..Also...großzügig

P2. Nein, nein, penibel ist, ... ist..., ist... der Gegenteil von...

P3: Penibel ist pingelig

K: Ergänzen Sie: was für den Türken sein Schnurrbart ist, ist für den Deutschen sein...

P1: Was würde ich sagen? Die deutsch... typisch deutsch... kein Schnurbart.

K: Kaya nickt verständnisvoll und notiert die Antwort. Kein Schnurrbart Was heißt pingelig? Wenn man oft aufs Klo gehen muss und...?

P2: Nein, nein.. Doch, penibel ist Sauberkeit.

$\mathrm{K}$ : Aha, pingelig ist, wenn es nicht so sauber ist! Kaya nickt verständnisvoll und notiert die Antwort.

${ }^{45}$ pin|ge|lig <Adj.> [rhein., westniederd. Nebenf. von peinlich] (ugs.): übertrieben gewissenhaft; pedantisch genau: ein sehr -er Mensch; dort ist es p. sauber; nicht sehr p. sein; p. mit etwas umgehen; wie p. die im Betrieb sind, wenn auf dem Fahrtenschreiber zwei Stunden Aufenthalt stehen ohne Grund (v.d. Grün, Glatteis 70); sei doch nicht so p.!; die -e (buchstabengetreue) Auslegung eines Paragraphen. 2000 Dudenverlag

${ }^{46}$ pe|ni|bel $<$ Adj.; ...bler, -ste $>$ [frz. pénible= mühsam; schmerzlich, zu: peine $<$ lat. poena, Pein] (bildungsspr.): a) bis ins Einzelne so genau, dass es schon übertrieben od. kleinlich ist: eine penible Ordnung; Die peniblen Prüfer ermittelten jedoch, dass es 880 Mark sein müssten (Szene8, 1984, 25); er ist in Geldangelegenheiten überaus, schrecklich p.; Seine Notizen ... übertrug er dann p. mit gestochener Schrift in zwei verschiedene Büchlein (Süskind, Parfum 118); b) (landsch.) peinlich (1): dass ... die in der Rückrunde unbesiegte Equipe einen enttäuschenden, sogar peniblen Eindruck hinterließ (NZZ 3.5. 83, 29). 2000 Dudenverlag 
Warum, glauben Sie, haben die deutschen Männer bei ausländischen Frauen so viel Erfolg? Also, bei uns, in der Türkei sind deutsche Männer sehr beliebt.

P1: Ehrlich?

$\mathrm{K}: \mathrm{Ja}$, glauben Sie mir.

P2: Das glaube ich nicht.

K: Glauben Sie nicht?

P2: Was ist denn mit verheirateten und und und..das weiß ich nicht $\mathrm{K}$ : Also, pingelig hat nichts zu tun mit aufs Klo gehen?

P1: Nein, nein, nein

P2: Nein

Es kommt eine Frau auf ihn zu und überrascht ihn mit dem Satz.

P4: Wissen Sie, wem Sie ähnlich aussehen?

K: Nein.

P4: Dem Komiker, der sag† "Was guckst du?" Kaya lächelt.

$\mathrm{K}: \mathrm{Ja}$, da gibt's einen. Kaya ist überrascht, lächelt und gibt nicht zu wissen, dass es um ihn geht

P4: Sie sind ihm ähnlich.

$\mathrm{K}$ : Momentan, wir sind vom türkischen Schlag alle gleich!

P4: Ja, aber gut! Kaya ist überrascht, lächelt und gibt nicht zu wissen, dass es um ihn geht

$\mathrm{K}: \mathrm{O}$, finden Sie inn gut?

P4:Ja! [...]

Das Format der Passantenbefragung erscheint in vielen Folgen von „Was guckst du?", weil die Autoren auf diese Weise auch den sog. Menschen von der Straße in das Programm mit einbeziehen, so dass die dargestellten Themen glaubwürdiger werden können.

Die auf das Alltagsleben bezogenen Fragen widerspiegeln Probleme verschiedener Art, auf die die Ausländer in Deutschland stoßen - von den Sprachproblemen, über die Schwierigkeiten in Kontakten mit den Deutschen, bis zu den amtlichen Angelegenheiten.

Die deutsche Szenerie trägt dazu bei, dass die in der Sendung auftauchenden Figuren leicht stilisiert werden können. Den intertextuellen Charakter der Sketche garantieren die auf die Fernsehshows stilisierten Szenen (mit Bezug auf „Wer wird Millionär?“, „Jeopardy“), die Figuren, die den Helden der Filme ähneln, wie z.B. ein türkischer Polizist im Film „Der Türke von Tölz“ (mit Bezug auf die Krimiserie „Bulle von Tölz“) oder die „Aktion Sorgenrind“ von Ranjid, die auf die in Deutschland seit Jahren organisierte „Aktion Sorgenkind“, zurückgeht. Da der Sketch als eine Performance zu verstehen ist, verbindet er das Sprachliche mit 
dem Außersprachlichen, so dass die an die Szenen angepasste Lexik meisterhaft mit den nonverbalen Elementen ergänzt wird.

Der Sketch als eine besondere Art Witz erscheint vor allem in Form eines Monologs, in dem die Pointe in erster Linie aus der Sprache und andererseits aus dem situativen Kontext hervorgeht, manchmal ist es sogar der Fall, dass eben das Nonverbale zum richtigen Entschlüsseln der versteckten lustigen Inhalte beiträgt. Die Komik wird mit den von den Autoren stilisierten Figuren, ihren Erscheinungsbildern, Körperbewegungen und ihrer Sprache zum Ausdruck gebracht. Jede Figur hat ihr eigenes Potenzial, das Humoristische zu erzeugen und den Rezipienten zum Lachen zu bringen.

Die in „Was guckst du?“ angebotenen witzigen Dialoge haben eine Pointe, die auch mit der Mimik der Darsteller vorher erkennbar sein kann, so dass der Zuschauer ungeduldig darauf wartet, was gleich gesagt wird. Es darf nicht außer Acht gelassen werden, dass im Falle der Sketche das mit dem Darstellen zusammenhängende Dramatische die Hauptrolle spielen kann. Dabei ist eben die übertriebene Darstellung der stilisierten Figuren der Vertreter verschiedener Nationen das Hauptinstrument, mit dem diese Aufgabe gelöst wird. Den Autoren und den Darstellern gelingt es, im Rahmen der Sendung auch darauf hinzuweisen, wie schwer es für einen aus einem ganz anderen Kulturkreis kommenden Ausländer ist, sich der deutschen Realität anzupassen. Es werden das deutsche Gesprächsstil, die Amtssprache, die angebliche Ordentlichkeit der Deutschen im Zerrspiegel präsentiert und auf diese Weise auch ausgelacht.

\subsection{Ethnowitz in „Was guckst du?“6}

Nach der in diesem Buch besprochenen Analyse der ausgewählten Sketchtexte aus "Was guckst du?" kann man zu dem Schluss kommen, dass zwischen der in der Sendung angebotenen Art Humor und dem sog. typisch deutschen Sinn für Humor sowohl Gemeinsamkeiten als auch Unterschiede bestehen. Yanar als Mitautor und Moderator der Show führt die Zuschauer in eine interessante, ihnen bisher fremde Welt anderer Kulturen, Traditionen und Mentalitäten. Das alles wird jedoch nicht als eine Kritik verstanden, sondern eher als Präsentation mit viel Sympathie und Humor. Die Sprache der Show, die sich vor allem auf die arabische Kultur beruft, voll von fehlerhaften Formen ist, die die Autoren mit Absicht einsetzen, bildet ein gutes Untersuchungsmaterial für die Analyse unter dem Aspekt des Humoristischen. Die Autoren der Sendung betonen selbst, dass die Sprachen im Zentrum ihres Interesses stehen, was auf ihre Charaktereigen- 
schaften zurückgeht, die nur einer konkreten Nation zugeschrieben stehen. Yanar betont in einem seiner Interviews ${ }^{47}$ :

Sprachen haben Charaktereigenschaften [...] Eine Sprache kann ja auch ein Lebensgefühl rüberbringen. Das Wort „Merde“ heißt auf Deutsch „Scheiße“, aber wie hört sich das an: Scheiße! [...] Aber Merde könnte auch, wenn man kein Französisch kann, eine Vorspeise sein.

Man greift zur fremden Aussprache und zu den Elementen des AusländerDeutschen, was jedoch von Yanar als nichts Negatives betrachtet werde, da es ihn an seinen Vater erinnere [Neumayer 2007].

Wenn man versucht, die kulturellen Hintergründe einer lustigen Sendung zu finden, zu nennen und schliesslich auch zu analysieren, dann soll man natürlich auch im Auge behalten, wie unterschiedlich sie sein können, was sich mit dem Charakter der jeweiligen Nation und ihrer Mentalität erklären lässt. Dabei darf das oft genannte und kritisierte, für jede einzelne Nation andere, nur für sie charakteristische Tabu nicht vergessen werden, das entweder in den witzigen Texten ausgelacht wird oder aber sogar nicht ausgelacht werden darf.

Grundsätzlich sind die kulturellen Gemeinsamkeiten beim Humor stärker ausgeprägt als die Unterschiede. Letztere sind schwer festzumachen, wenn auch manchmal spürbar und empirisch nachweisbar. So variiert etwa der Umgang mit Ironie, wenngleich es dieses Stilmittel in allen Kulturen gibt.[...] Je nach Region verlagern sich auch die Tabugrenzen. So arbeitet englischer Humor eher mit Ekel als der deutsche. Die Briten machen auch öfter den Tod zum witzigen Gegenstand als Deutsche. Besonders gut sind solche Differenzen auch hinsichtlich der Beziehung von Humor und Religion erkennbar: in der christlichen und vor allem in der jüdischen Kultur etwa haben Witze über Gott eine lange Tradition; in der muslimischen Kultur gibt es sie nicht. Witze über religiöse Inhalte und muslimische Geistliche dagegen schon. ${ }^{48}$

Die Sprache der Figuren von „Was guckst du?“ ist eine gelungene Hybride, die aus den Elementen des Deutschen und den der anderen Sprachen besteht (Türkisch, Arabisch, Russisch, Italienisch), was wiederum mit dem Herkunftsland der jeweiligen Figur (u.a. türkischer Türsteher Hakan, Italiener Francesco, türkischer Fahrlehrer Yildirim, russische Hellseherin Olga, arabischer Journalist Tarek ab del Kalek, Ranjid aus Indien) zusammenhängt. Es kann hier die Rede von der ,[...] wesentlich durch Mischsprachen geprägten interkulturellen Kommunikation, verstanden als öffentliches Gespräch unter Angehörigen verschiedener Szenen oder

${ }^{47}$ Vgl. dazu J.Wittner, Interview Kaya Yanar. Merde in Germany, von stern.de/ unterhaltung - 5.3.2008 (=http://www.stern.de/kultur/tv/interview-kaya-yanar-merde-in-germany-613128.html).

${ }^{48} \mathrm{Vgl}$. dazu Interview mit H. Kotthoff, Die Humorforscherin: Lachen, theoretisch, von : fudder.de (=http://fudder.de/artikel/2008/01/21/die-humorforscherin-eher-sach-als-lachgeschichten/) 
Subkulturen mit unterschiedlichen Soziolekten [...]“ sein [Hübner 2006]. Yanar selbst äußert sich nicht nur in einem seiner zahlreichen Interview dazu, wie wichtig für seine Show die Sprachen verschiedener Nationen sind, deren Elemente er geschickt in die Texte einsetzen kann, um auf diese Weise ein bestimmtes Kolorit der Sendung zu unterstreichen. Nicht weniger wichtig ist dabei die Tatsache, dass er auch nach dem gemeinsamen Punkt sucht, wo sich sein Sinn für Humor und sein Geschmack mit dem seines Publikums überlappen.

Die Konfrontation der enstandenen Hybridesprache mit dem Deutschen lässt auf folgende Merkmale der für die Sendung charakteristischen Sprache hinweisen, d.h.

- Fehler in der Flexion

- Fehler im Bereich der Syntax

- Polysemie der gebrauchten Lexeme und das fehlende Wissen über die jeweilige Bedeutungsvariante

- Gebrauch der Elemente der Fachsprache

- Gebrauch der Elemente fremder Herkunft

Diese Art Sprache - broken german - ist nicht nur für die Sendung „Was guckst du?“ charakteristisch. Auch andere Komiker, die die so genannte Ausländer-Comedy machen, wie z.B. Erkan\&Stefan, bedienen sich dieser Form beim Schreiben lustiger Texte für die Bühne. Der Sendung „Was guckst du?““ wurde jedoch eine besonders wichtige Rolle im deutschen Fernsehen zugeschrieben, was auf ihren multikulturellen Charakter zurückzuführen ist. In der Geschichte der deutschen Unterhaltung war es die erste Sendung, in der nicht die Deutschen, sondern Einwanderer mit viel Sympathie und nur wenig Biss präsentiert werden. Diese Gruppe umfasst verschiedene Nationalitäten, was dazu beiträgt, dass „Was guckst du?" als Sendung mit einer besonderen Rolle verstanden werden kann - als solche, deren Aufgabe ist, das Multikulturelle wiederzugeben, zu präsentieren und vor allem die deutschen Zuschauer damit bekannt zu machen. Der Moderator bemüht sich dabei um eine authentische Darstellung der jeweiligen Figur, ihrer Identität und Mentalität [Schröder 2001]:

Meine Art von Humor ist anders. Bei den Imitationen, in die ich schlüpfe, bemühe ich mich schon um die Authentizität. Natürlich spiele ich mit Klischees, sonst wäre das nicht lustig, aber das ganze ist noch mal so authentisch, weil ich selbst halb Araber, halb Türke bin. Und ich reize halt keine Figur aus, sondern springe von einer Rolle in die nächste. Es wird ständig gewechselt zwischen Italienern, Arabern, Deutschen, Indern und das ergibt dann so ein lustiges Konglomerat.

Die Sendung „Was guckst du?“ wird als Ethno-Comedy klassifiziert, der eine integrierende Funktion zugeschrieben steht. Ein breites Spektrum von Figuren aus 
verschiedenen Nationen ermöglicht eine ziemlich scharfe Kritik an der deutschen Gesellschaft auszuüben, wobei „Was guckst du?“ eine gelungene Mischung aus Sketchen ist, in denen nicht nur eine bestimmte Nation, sondern viele verschiedene ausgelacht werden. Yanar selbst bezeichnet seine Art Humor als ,, nicht von oben herab, sondern auf einer Ebene mit dem Publikum " [Neumayer 2007] und seine Sendung versteht er als ,,eine Art Liebeserklärung an die Nation “49.

In der Sendung „Was guckst du?“ werden verschiedene Nationen im Zerrspiegel dargestellt, vor allem anhand dieser bis heute noch geltenden Stereotype. Im Folgenden werden die repräsentativsten Sketchbeispiele aus „Was guckst du?“ unter dem Aspekt der früher genannten nicht-muttersprachlichen Varietäten der deutschen Sprache besprochen. Die von Yanar selbst dargestellten Figuren bedienen sich einer besonderen Sprache, d.h. des Ausländerdeutschen, sei es ItalienerDeutsch, Polen-Deutsch, Arabisch-Deutsch oder aber Türkisch-Deutsch ${ }^{50}$

In „Was guckst du?“" werden Italiener vor allem als Angeber kritisiert.

\section{(60) Szene im Zimmer von Kaya Yanar}

Kaya unterhält sich mit Francesco

Francesco: Was ist so wichtig, (60a) dass du mich hat wegholen von einem Essen mit (60aa) bella signorina?

Kaya: (60b) Du bist bestimmt wieder in die Hose gegangen. Francesco: (60c) Stimmt es nix! Bin ich gar nicht in die Hose gegangen, aber bis Bluse...

Kaya: Hör zu, ich habe drei junge Damen eingeladen, die an einer Show mit Herzblatt spielen werden.

Francesco: Ich darf in deiner Sendung spielen mit drei jungen Damen? (60d) Was ist genau für eine Sendung, die du hie machst? Kaya: Nein, nein! Du solltest dir nur eine aussuchen. Hier! Ich habe dir Fragen schon völlig aufgeschrieben. Bis später.

Francesco: Kandidatin eins, ich bin Italiener. Kannst du ein bisschen Italienisch? Wieso Italienisch??? (60e) Besser ist es, viel Französisch...

In diesem Dialog fällt vor allem die sog. Ausländer-Deutsch-Variante auf, weil Francesco ein gebrochenes Deutsch spricht, das noch zusätzlich mit den ita-

${ }^{49}$ Kaya Yanar erzählt: „Liebeserklärung an die Nation“, von www.kino.de (=http://www.kino. de/news/liebeserklaerung-an-die-nation/165950)

${ }^{50}$ Dabei muss betont werden, dass es im Falle dieser Sendung kein richtiges Kanak-Deutsch ist, nur ein sog. broken german, was schon bei Kotthoff (2004c) erwähnt wurde. 
lienischen Elementen vermischt wird (60aa - bella signorina). Es können hier die Fehler im Bereich der Wortfolge gefunden werden (60a - *dass du mich hat wegholen und 60d*Was ist genau für eine Sendung) und in demselben Abschnitt tauchen zwei grammatische Fehler auf (60a - *du hat wegholen). Die von Yanar gespielte Figur - Francesco - formuliert auch Sätze, in denen nicht richtig erlernte Lexik auftaucht (60c - *bin nicht in die Hose gegangen, aber bis Bluse) und zum Schluss betont er noch seine Vorteile als Liebhaber (60e Wieso Italienisch??? Besser ist es, viel Französisch), wenn er an eine Bedeutungsvariante des Wortes Französisch im Sinne von 'französische Liebe, Oralverkehr' denkt.

In den Ethnowitzen werden, wie Yanar selbst behauptet, alle Nationalitäten gleich kritisch und ironisch, aber dabei nicht bissig behandelt, auch Polen, wobei man zum Vorurteil greift, dass jeder Pole ein Dieb ist [Sikorska-Bujnowicz 2011a].

\section{(61) Szene auf dem Polizeirevier}

Ein Pole, namens Olszewski, will eine Anzeige erstatten, denn jemand hat ihm sein Auto geklaut.

Polizist 1: Ihr Name?

Pole: Olszewski

Polizist 1: Nationalität?

Pole: Pole

Polizist 1: Und? Warum möchten Sie die Anzeige erstatten?

Pole: (61a)Hat man mir geklaut meine Auto.

Polizist 1: Hehe

Pole: (61b)Was los?

Polizist 1: Heheh, Tschuldigung, es ist nur, ein Pole, dem man das Auto geklaut hat. Egal. Haben Sie schon irgendwelche Hinweise auf den Täter?

Pole: Nein.

Polizist 1: Haben Sie schon bei mal bei Ihren Bekannten in Polen nachgefragt? Vielleicht haben die ja, naja. Waren denn im Wagen noch irgendwelche persönlichen Besitztümer?

Pole: Persönliche was??

Polizist 1: Naja, Sachen, die Ihnen gehören. Tasche, Hut, ... ein Brecheisen

Pole: Jetzt habe ich aber genug. Willst du damit sagen, dass (61c) jede Pole ist eine Dieb?

Polizist 1: Schon gut, tut mir leid. Die dummen Vorurteile, Sie wissen schon. Hier. Sie müssen noch einmal unterschreiben

Pole: Also (sucht sich ab, hat keinen Kugelschreiber) 
Polizist 1: Nehmen Sie meinen. (Olszewski unterschreibt und verschwindet)

Polizist 2: Was?!

Polizist 1: Ah, nichts. Bin noch mal kurz weg. Mir n neuen Kuli kaufen. Wo ist mein Portemonnaie? Und meine Schreibmaschine? Heinz, hast du?

Polizist 2: Nee. (sitzt nackt am Schreibtisch)

Der angeführte Sketchtext hat die Form eines Dialogs zwischen einem Polen und einem deutschen Polizisten. In diesem Fall werden sprachliche Mittel zum wichtigen Mittel des Humoristischen. Die Sprache des Polen ist voll von Fehlern, zu denen folgende fehlerhafte Formen gehören: falsche Wortfolge (61a) - an erster Stelle in diesem Aussagesatz erscheint das Prädikat, und (61c) - falsche Wortfolge nach dass, das Weglassen des Kopulaverbs (61b - *was los), zweimal falscher Artikelgebrauch (61c - *jede Pole und *eine Dieb). Das Komische betonen in dem Gesamtkontext auch die Worte des Polizisten Haben Sie schon bei mal bei Ihren Bekannten in Polen nachgefragt? Vielleicht haben die ja, naja ... , mit denen gemeint wird, dass Polen eine klauende Nation sind. Der situative Kontext hat hier eine ergänzende Rolle zu spielen, weil damit gezeigt wird, dass sogar Polizisten auf ihrem Revier beklaut werden können

Auch die Türken erscheinen als Zielscheibe in den Sketchen von Yanar, indem er auf ihre Sprech- und Sprachprobleme zeigt.

\section{(62) Szene im "Radio Süleyman"}

Süleyman: Hallo, Leute! Ich bins wieder, Süleyman. Heute (62a) aus provisorische Studio, (62b) naturlich mit meine beste Freund Süleyhund. Süleyhund, (62c) kommst du! So, erster Anrufer. (62d) Was gibt's oder gibt's nix?

Süleyman spricht broken german, entweder kennt er die Regeln der deutschen Grammatik nicht bzw. verstößt gegen sie, was die von ihm gemachten Fehler bestätigen, unter denen die Deklinationsfehler (62a) - *aus provisorische Studio, (62b) *mit meine beste Freund und die bei der Bildung des Imperativs (62c) *kommst du! genannt werden können. Es gibt auch einen Aussprachefehler (62b) *naturlich. Die nicht mit der Intonation eines Fragesatzes ausgesprochene Frage (62d) )Was gibt's oder gibt's nix? dient als Begrüßung der Anrufer und soll sie zum Sprechen ermuntern. Der zitierte Satz wird somit zum Erkennungszeichen dieser Figur. 
Eine andere türkische Gestalt ist Hakan, der Türsteher, mit seinen bekannten Sätzen Ey, du, du kommst hier nicht rein! oder Ey, du, willst du Stress oder was? oder aber Ey, Alder, mach mal locker!, in denen die typischen Elemente des Türkisch-Deutschen auftauchen, d.h. Ey, $d u$ ! und Ey, Alder! Diese werden in allen Szenen mit Hakan gebraucht.

Im Rahmen von „Was guckst du?" wird auch gezeigt, wie man auf die Sprachprobleme eines Ausländers in Deutschland reagiert und wie man diesem dabei hilft, wobei eben diese Hilfsversuche der Deutschen dem Ausländer gegenüber und der sich dabei entwickelnde sprachliche und außersprachliche, sowohl der situative als auch der mit der Körpersprache der beiden Gesprächspartner zusammenhängende Kontext, zur Herausbildung des Humoristischen führen. Der im Folgenden präsentierte und besprochene Sketch könnte als Ausgangspunkt und zugleich als Zusammenfassung der von mir in diesem Buch angesprochenen Problematik dienen. Die Autoren platzieren die Szene in der deutschen Realität - in der Fußgängerzone einer deutschen Stadt, der Sketch hat die Form einer Passantenbefragung und der Zuschauer muss nur einfach abwarten, was sich aus diesem „Experiment" ergibt - ob die darzustellende Realität tatsächlich im Endeffekt eine komische Wirkung haben kann, wie die Reaktionen der Befragten sind, was ihre Körpersprache signalisiert - ist das Sympathie, Abneigung oder vielleicht Gleichgültigkeit? Eine einfache Antwort auf die so formulierte Frage hängt einerseits davon ab, wie der sprachliche Kontext konzipiert ist, welcher Mittel man sich bedient, um eben die Doppeldeutigkeiten zu erzeugen und andererseits ist das alles mit den manchmal gar nicht erwarteten Reaktionen der befragten Passanten verbunden, was dazu führt, dass der Zuschauer lacht, was wiederum die Aufgabe des Witzes erfüllt.

\section{(63) Ein Ausländer befragt die Passanten}

Kaya (K) steht, ein Wörterbuch in der Hand haltend, auf der Straße und fragt die Passanten (P1, P2) nach verschiedenen Sachen.

Kaya: (63a)Entschuldigen, ich habe eine Frage...

K: (63b) Wo ... kann man... hier gut... Er fängt an, im Wörterbuch nachzuschauen.

P1: Langsam...

$\mathrm{K}:$...(63c) Popel essen?

Das Gespräch wird unterbrochen und wir sehen den zweiten Fußgänger, der gefragt wird.

K: (63d) Wo ist hier... die Toilette... machen?

P2: (63e) Machen nichts. Machen nichts. 
K: Doch, doch!

P2: Sie müssen zur Toilette.

K: Hose

P2: (63f) Wenn Sie nicht rechtzeitig auf die Toilette, dann geht es in die Hose.

Eine vorbeigehende Frau wird gefragt

K: (63g) Entschuldigen. (63h) Wo ... kann man hier in den Rhein schiffen?

Sie ist überrascht, geht weiter, ohne ein Wort zu sagen.

An dieser Stelle wird der Dialog mit zwei früher erwähnten Passanten fortgesetzt.

P1: Da kommt unten aus, aus (der Mann gestikuliert viel) eine Säule (am Regenschirm will er dem Ausländer zeigen, was eine Säule ist und macht dabei eine mehrdeutige Geste, die mit der Selbstbefriedigung der Männer assoziiert wird. Kaya lacht.)

P1: Ja, Hohe Straße, ja... (schaut den Kaya an, um sich zu vergewissern, dass er ihn verstanden hat)

K: Säule (Kaya wiederholt die vorher von dem Passanten gemachte Geste), essen, (63i) nichts Säule (lacht weiter)

An dieser Stelle wird der Dialog mit dem netten älteren Mann fortgesetzt.

P2: Da ist die Toilette.

K: (63j) Dort Hose

P2: Da ist die Toilette.

$\mathrm{K}$ : Ok, danke schön!!

Wieder tauchen fehlerhafte Formen auf, wie falsche Verbform (63a),(63g)*Entschuldigen, Fehler beim Gebrauch des Negationswortes (63e)*machen nichts, (63i)*nichts Säule und eine fehlerhafte Zusammenstellung von zwei Wörtern (63j)*Dort Hose. Es wird auch die Lexik gebraucht, die zwar im Wörterbuch zu finden ist, leider aber nicht richtig verstanden wird (63c)*Popel essen, (63d)*die Toilette machen nicht im Sinne von 'sich frisch machen', sondern als 'auf die Toilette müssen' und im Falle der Form (63h)*in den Rhein schiffen liegt die veraltete Bedeutung des Verbs vor.

Beim Schreiben der Texte wird die deutsche Szenerie gewählt, weil auf diese Weise die Alltagsprobleme der Ausländer in Deutschland glaubwürdiger dargestellt werden können. Diese werden u.a. bei der Arbeitssuche gezeigt, indem ihr Verhalten während eines Vorstellungsgesprächs, vor allem aber die Probleme mit der deutschen Sprache ausgelacht werden, wobei auch darauf angespielt wird, dass es unter ihnen auch solche gibt, die der deutschen Sprache auch im Rahmen der offiziellen Amtssprache mächtig sind, was im folgenden Sketch auffällt. 
(64) Türkischer Dolmetscher beim Bewerbungsgespräch

Wir sehen einen Deutschen im Büro sitzen. Er ist Berufsberater (B). Zwei Leute kommen herein (T1 als Dolmetscher und T2 als Arbeitsuchender, Herr Elgül)

B: Herein! A, Herr Elgül... und wer sind Sie?

T1: Sein Bekannter. Ich übersetze.

B: Ah, ja. Es soll diesmal besser gehen. Nehmen Sie Platz.

T1: Danke schön.

B: Ok, dann übersetzen Sie mal, bitte. Guten Tag, Herr Elgül!

T1: (64a) Hey, Alde! Sie begrüßen sich mit den Gesten wie die Mitglieder einer Subkulturgruppe.

B: Sie arbeiten seit 14 Jahren als Schlosser.

T1: (64b) Ey, bist du Schlosser oder was? Er verzieht das Gesicht.

T2: Das weißt du doch!

T1: Ja.

B: und plötzlich hat Ihnen Ihr Arbeitgeber gekündigt

T1: $(64 c)$ und dann haben sie dich die Schweine den Arschtritt vor die Tür gejagt

T2: (64d) Dann sag doch, die Arschlöcher, ja!

T1: Ja.

B: Aus welchem Grunde ist das passiert?

T1: Wieso?

T2: (64e) Ich habe Bohrmaschine geklaut.

T1: Herr Elgül und seine Arbeitgeber hatten gewisse Differenzen bezüglich seines Gehaltes und da seine Arbeitgeber auf wiederholte freundliche Schreiben von Herrn Elgül verständnislos, um nicht zu sagen, schroff reagierten, hat Herr Elgül, obwohl er eine sehr große Stütze für seinen Betrieb ist, ...war, ein weinig zu viel Eigeninitiative entwickelt. Das gefiel seinen Arbeitgebern überhaupt nicht und sie haben ihm deswegen gekündigt.

B: Hmm, verstehe.

T1-T2: (64f) Korrekt! Sie klatschen einander ab.

B: Ja..., leider habe ich im Moment keine Angebote für Schlosser, aber könnensie sich vorstellen, als Packer zu arbeiten?

T1: $(64 \mathrm{~g})$ Ey, willst du Scheißjob als Packer oder was?

T2: Wie viel? Mit dem Reiben der Finger der rechten Hand wird von ihm auf das Geld hingedeutet.

T1: Wie sind dann die Verdienstmöglichkeiten?

$B: 0$, da vertut man sich. Moment... Ja... Er blättert die Unterlagen durch. Wenn Sie die Zulagen, Weihnachtsgeld und Wochenendausgleich berücksichtigen, dann kommen Sie auf 4500 brutto. 
T1: (64h) Sie wollen dich verarschen. (64i) Das ist Scheißdreck-arsch-scheißjob, keine Kohle, Dreckkohle, Scheiße.

T2: Er fängt an, auf türkisch zu schimpfen und verlässt den Raum.

B: Was ist denn jetzt passiert? Was haben Sie gesagt?

T1: Ich habe natürlich nur das übersetzt, was Sie gesagt haben. Aber meine andere Frage: kann ich den Job haben?

$\mathrm{B}: \mathrm{Ja}$, natürlich.

Im oben zitierten Text fallen zwei deutsche Sprachvarietäten auf - das Hochdeutsch (Beamter und Türke1) und das Türkisch-Deutsch. Im Gespräch zwischen den beiden Türken wird nur broken german gebraucht, was gleichzeitig mit dem Dolmetschen der Worte des Beamten zusammenhängt (64a) Hey, Alde!, (64b) Ey, bist du Schlosser oder was?, (64c) Und dann haben sie dich die Schweine den Arschtritt vor die Tür gejagt., (64d) Dann sag doch, die Arschlöcher, ja! (64f) Korrekt!, (64g) Ey, willst du Scheißjob als Packer oder was? (64h) Sie wollen dich verarschen. Mit Hilfe der Komposition entstehen die Neubildungen in Form von mehrgliedrigen Zusammenbildungen, wie (64i) Scheiß-dreck-arsch-scheißjob.

In diesem Sketch werden synonyme Strukturen für Beamtendeutsch im Rahmen des Türkisch-Deutschen gebraucht. Bei der Bildung neuer Lexeme als Äquivalente für hochdeutsche Wörter spielt das Emotionale die entscheidende Rolle, weil dabei neutrale Grundwörter mit den vulgären oder saloppen Elementen kombiniert werden (Job vs. Scheiß-dreck-arschscheißjob). In der Konstruktion (64e) Ich habe Bohrmaschine geklaut. fehlt der Artikel. Erst im Zusammenhang mit dem Text tragen die außersprachlichen Elemente zur Entstehung des Komischen bei. Das Äußerungsbild der beiden Türken, verbunden mit dem stereotypen Bild dieser Nation, die Verhaltensweise der beiden, die Gegenüberstellung ihrer Idiolekte und Soziolekte ergeben im Endeffekt eine interessante Mischung des Sprachlichen und des Außersprachlichen, was die Rezipienten zum Schluss lachen lässt, weil die beiden Figuren in der Opposition zueinander stehen. Der Dolmetscher bedient sich einerseits fließend der deutschen Hochsprache und Amtssprache, andererseits kommuniziert er mit seinem Freund mit Hilfe des Türkisch-Deutschen. Diese Szene betont auch die Bedeutung der Soziolekte innerhalb der Einwanderergesellschaft, weil es dabei um ihre Funktion innerhalb einer fremden Kultur geht - sie sind ein Verständigungsmittel, das gleichzeitig fast wie eine geheime Sprache zu sein scheint.

$\mathrm{Zu}$ der türkischen Kultfigur aus „Was guckst du?“ ist Hakan, der bereits erwähnte türkische Türsteher, geworden, für den der Spruch 65a-c Ey, $d u, d u$ kommst hier nicht rein! charakteristisch ist. 


\section{(65) Werbung für Dreier-Fahrt}

Wir sehen Hakan, der aus seinen langen Haaren eine merkwürdige, aber doch perfekt haltende Frisur gemacht hat.

20:00 Szeneladen,

Hakan: (65a) Ey du, du kommst hier nicht rein! Die Frisur hält. 23:00 Discoeinlass, Hakan: (65b) Ey du, du kommst hier nicht rein! Die Frisur hält. 1:00 Coctailbar, Hakan: (65c) Ey du, du kommst hier nicht rein! Perfekter Halt dank Dreier-Fahrt, denn nur die Dreier-Fahrt gibt Ihrer Frisur den perfekten Halt für den ganzen Tag.

In dieser kurzen Szene fällt die Anspielung auf die Werbung für die Haarkosmetikreihe Drei-Wetter-Taft auf. Es ist aber nicht die Werbung für den Haarlack, sondern für den BMW. Auch hier wird von dem Rezipienten das Vorwissen verlangt, damit von ihm auch der intertextuelle Charakter und somit der Sinn dieser Szene verstanden werden können, denn oft kommt es vor, dass das fehlende Wissen aus einem bestimmten Bereich das Finden, Entschlüsseln und Interpretieren der versteckten lustigen Inhalte erschwert, manchmal sogar auch unmöglich macht.

Als Paradebeispiele der Szenen, wo die fehlenden Deutschkenntnisse bewusst zum Thema, Motiv und auch zur Zielscheibe werden, gelten die folgenden, in denen man bewusst u.a. mit der Polysemie des Verbs (66a) (sich)ausweisen ${ }^{51}$ spielt

${ }^{51}$ aus|wei|sen $<$ st.V.; hat $>$ : 1. des Landes verweisen, jmdm. nicht länger den Aufenthalt in einem bestimmten Land gestatten: einen Staatenlosen a.; jmdn. als unerwünschte Person a. 2. [mithilfe eines Ausweises (1)] seine, jmds. Identität nachweisen: bitte weisen Sie sich aus!; die Dokumente haben ihn als Unterhändler ausgewiesen; Ü der (=ein Anglist) zwar hochschulpolitisch noch kaum hervorgetreten, aber als Mitglied des Bundes Freiheit der Wissenschaft eindeutig ausgewiesen war (Nuissl, Hochschulreform 67). 3. a) <a. + sich> sich erweisen: sich als guter/(selten:) guten Geschäftsmann a.; wenn man in der Gruppe eine Aktionsform zu sehen bereit ist, die sich an Erfolgen auszuweisen vermag (Hofstätter, Gruppendynamik 21); b) unter Beweis stellen: sein Talent a.; Dieses Ansehen hat er sich mehr noch als durch sein ausgewiesenes Können durch die Furchtlosigkeit... erworben (Böll, Und sagte 152 [Nachwort]); c) <a. + sich> (schweiz.) (Kenntnisse, Fähigkeiten) nachweisen: Der Bewerber muss sich über eine abgeschlossene handwerkliche Berufslehre a. können (Basler Zeitung 9.10. 85, 50); d) <a. + sich $>$ (schweiz.) beweisen (2): Weltrekordhalter Bubka wies sich über Nervenstärke aus (NZZ 31.8. 86, 33); der Platzklub ... wies sich auch über eine gute Raumaufteilung aus (NZZ 2.9. 86, 37). 4. rechnerisch nachweisen, zeigen: wie die Statistik ausweist; amtliche Register wiesen aus, dass sie an diesem Tage in Paris geweilt hatten (Mostar, Unschuldig 25); ausgewiesene Überschüsse. 5. (Bauw.) für einen bestimmten Zweck vorsehen, zur Verfügung stellen: Zu den noch verfügbaren Flächen wurden schon neue Gebiete ausgewiesen, die in fünf bis zehn Jahren verkauft 


\section{(66) Polizeikontrolle 1}

Ein Türke geht einem Polizisten entgegen. Wenn sie schon aneinander vorbeigehen, fragt der Polizist

\section{P: Können Sie (66a) sich bitte ausweisen? \\ Darauf antwortet der Türke \\ T: Wieso sollte ich? Ich lebe gerne in Deutschland.}

oder mit der Bedeutung des Verbs durchfallen ${ }^{52}$ spielt, das der Wendung Durchfall $^{53}$ kriegen gleichgesetzt wird, was im Endeffekt eben zur Bildung des lustigen Kontextes verhilft

\section{(67) Fahrschule Kelal Yildirim 4}

Fahrlehrer Yildirim spricht mit seinem deutschen Schüler Manfred Winter

\section{Yildirim: Wenn du willst Auto fahren lernen wie ein Türke, du musst anfangen türkisch zu denken. Sonst (67a) kriegst du Durchfall.}

werden können (Hamburger Abendblatt 23.5. 85, 23); obwohl auch hier der Bebauungsplan ... eine ganz andere Bebauung auswies (Stuttg. Zeitung 14. 10. 89, 34). 6. a) offiziell als etw. bezeichnen, zu etw. erklären, deklarieren: Auch das Saarland unterstützt eine Initiative des Bundesrats, wonach der Ausbau der A8 ... als Bedarf ausgewiesen wird (Saarbr. Zeitung 28. 12. 79, 24); Die einen erhoffen sich Entschädigungen, die anderen ein Gutachten, das ihre Häuser als einsturzgefährdet ausweist und staatliche Mittel verheißt (Fest, Im Gegenlicht 246); b) kennzeichnen, angeben: mit der Angabe der Verbraucherfrist, sie wird seit November 1986 generell ausgewiesen, kommen wir den Wünschen der Kunden entgegen (Freie Presse 17. 11. 83,3); die Kosten für Eltern und Kinder werden nicht separat ausgewiesen (a\&r 9, 1998, 136). 2000 Dudenverlag

${ }^{52}$ durch|fal|len <st.V.; ist> [2: urspr. Studentenspr.; geht auf den ma. Schwank vom Schreiber im Korbe zurück, in dem ein Mädchen seinen Liebhaber zum Fenster hochzieht, um ihn dann durch den schadhaften Boden fallen zu lassen]: 1. durch eine Öffnung hindurch nach unten fallen: die kleinen Steine fallen [durch den Rost] durch. 2. (ugs.) a) (von einem Theaterstück o.Ä.) keinen Erfolg haben: die Aufführung ist [beim Publikum] durchgefallen; Schon 167 v.Chr. fielen während der Triumphalspiele griechische Flötenspieler bei den Zuhörern durch (Thieß, Reich 355f.); b) (eine Prüfung) nicht bestehen: er ist [im Examen] durchgefallen; bei der Fahrprüfung d.; er ist mit Glanz durchgefallen (ugs.; hat in der Prüfung vollständig versagt); c) (bei einer Wahl) verlieren, nicht gewählt werden: der neu aufgestellte Kandidat ist bei der Wahl durchgefallen. 3. (Fliegerspr.) durchsacken: die Maschine... fiel eine Strecke weit durch wie ein Stein (Gaiser, Jagd 92). 2000 Dudenverlag

${ }^{53}$ Durch|fall, der; -[e]s, ...fälle [2: zu durchfallen (2)]: 1. [Krankheit, die gekennzeichnet ist durch die häufige] Ausscheidung von dünnflüssigem Stuhl; Diarrhö: D. bekommen, haben; eine mit schweren Durchfällen einhergehende Krankheit. 2. (ugs.) a) (von der Aufführung eines Theaterstücks o.Ä.) Misserfolg: das Stück wurde ein D.; Meine Komödie befremdete, missfiel. Es war ein D. (K.Mann, Wendepunkt 187); b) das Versagen, Nichtbestehen (bei einer Prüfung): groß ist die Panne ja nicht, die es zu beraten gilt, ein D. in der Schule (Frisch, Gantenbein 477); er hat beim Examen einen D. erlebt. 2000 Dudenverlag 
Es kann aber auch so sein, dass sich die Autoren ganz geschickt der falschen Wortfolge und des Sich-Versprechens bedienen, damit auf diese Weise die Schwierigkeiten mit der deutschen Sprache und die Unkenntnis der Bedeutung des mehrdeutigen Verbs durchfallen und des Substantivs Durchfall gezeigt werden. Das genannte Substantiv, das in Verbindung einen Durchfall erleben die erwünschte und gemeinte Bedeutung von 'nicht gelingen' hat, steht in der Wortgruppe (67a) Durchfall kriegen/bekommen weit entfernt von dem beabsichtigten Sinn.

Besonders im Falle von „Was guckst du?" liegt das sexuelle Witzeln vor, das in zahlreichen Szenen mit Francesco, der sich seiner Liebeleien rühmt, mit im Spiel ist.

Die Anmache mit viel Witz ist sein Markezeichen und wir sehen ihn im Restaurant, wenn er mit Frauen kurze Gespräche besonderer Art führt. Er ist frech und zu direkt. Das unerwartete Ende überrascht den Rezipienten, weil dieser mit der Frechheit von Francesco konfrontiert wird. Solche Art Witz ist für alle Szenen mit diesem italienischen Macho charakteristisch, in denen z.B. die Frau ausgelacht wird, weil sie angeblich zu viel zum Essen bestellt und zu schnell isst, z.B.

\section{(68) Francesco im Restaurant 1}

$F$ : $O$, bella, ich habe noch nie so eine Frau gesehen,

$\mathrm{B}: \mathrm{Ja}$ ?

F:... die so schnell essen kann er dreht seinen Kopf zur Seite .......... und wiederholt diese Kopfbewegung so viel Es sieht so aus, das er sich umsieht, ob jemand sie dabei beobachtet... und so teuer

oder nicht auf die Körperpflege achtet, weil sie nicht schön duftet

\section{(69) Francesco im Restaurant 2}

F: $O$, Bella, kannst du mir sagen, was ist das Parfüm, das du benutzt? Francesco zieht die Augenbrauen hoch. Die Frau versteht die Frage als Zeichen seines großen Interesses an ihr.

B: Wieso? Gefällt es dir? Die Frau ist überglücklich, dass er eben diesen Duft spürt.

F: No, no, no! Es überdeckt deinen Mundgeruch! Die Frau ist empört, steht auf und geht weg. Zahlen!

bzw. ihre lästige Beharrung nicht entfernt 


\section{(70) Francesco im Restaurant 3}

B: Zu dir oder zu mir? Die Frau lächelt verlockend.

F: Lieber zu mir... Von seinem Gesicht lässt sich aber keine Freude ablesen, eher das, dass gleich etwas Überraschendes gesagt wird.

B: Wieso? Die Frau erhöht die Stimme, zieht die Augenbrauen hoch, aber hört nicht auf zu lächeln. Man sieht ihre Überraschung wegen des Wortes lieber

F: Der Gesichtsausdruck von Francesco verrät böse Absichten - er lächelt seltsam, verzieht das Gesicht. Wegen meines Rasierapparates! Sie steht auf und geht weg.

oder aber wenn sie als Kandidatin für seine Ehefrau gelten kann, wenn sie seine Fragen beantwortet

\section{(71) Francesco im Restaurant 4}

$\mathrm{F}: \mathrm{O}$, Bella! Ich suche eine Frau, die nicht nur kochen kann...

B: Ja? Die Frau lässt sich auf dieses provokante Frage-Antwort-Spiel ein. Von ihrem Gesicht kann man ablesen, dass sie auf einen Heiratsantrag von Francesco wartet.

F: Sondern auch ... backen! Der Gesichtsausdruck ändert sich, die Frau ist empört, steht auf und verlässt das Restaurant. Zahlen! Francesco scheint aber zufrieden zu sein, zieht seine Augenbrauen hoch, bewegt seinen Schnurrbart, hebt die Hand hoch und ruft zum Kellner Zahlen!

und die Reaktion der Frau ist in jeder Szene gleich - sie ist entsetzt, steht auf und will weg und jedes Mal endet das Gespräch mit dem an den Kellner gerichteten Wort Zahlen! Die Szenen im Restaurant laufen nach demselben Schema und werden von denselben Dartsellern gespielt. Es fällt die italienisch-deutsche Variante der Sprache auf, d.h. die charakteristische Aussprache, der Gebrauch von italienischen Ausdrücken, wie Bella und das alles wird auf der außersprachlichen Ebene zusätzlich mit dem Erscheinungsbild dieser Figur betont, weil Francesco immer einen schicken Anzug anhat, frisch frisiert ist, ständig locker bleibt und schon dem Ausssehen nach ein Machotyp ist. Dabei spricht er viel und schnell, so wie in der stereotypen Darstellung eines Italieners der Fall ist. Den intertextuellen Charakter dieser Sendung bestätigt noch eine weitere Szene, in der Francesco an der Show „Herzblatt“ teilnimmt und seine Frau fürs Leben sucht. In diesem Fernsehformat wird der Sketch angeboten, in dem Francesco viele Fragen stellt, die ihm dabei helfen können, von drei Kandidatinnen nur eine zu wählen. Zum Schluss wird 
wieder auf Sex angespielt, indem Francesco eine Frage nach den Sprachkenntnissen stellt und überrascht feststellt Wieso Italienisch, besser ist viel Französisch! In dem zitierten Satz kann es um beides gehen - Kenntnisse des Französischen selbst und um die Kenntnis der französischen Liebe, also um das Sexuelle. So entsteht eben der Witz - der Zuschauer muss für sich entscheiden, was mit dieser Frage gemeint wurde und auf Grund seines Vorwissens, das sich unter anderem auch auf die stereotypen Bilder der einzelnen Nationen beruft, muss er konkrete Schlussfolgerungen daraus ziehen, um den Sinn der Aussage zu verstehen und den Witz als eine Einheit zu interpretieren.

Interessant ist es aber die Antwort auf die Frage, wie Witze dieser Art von den Rezipienten selbst eingestuft werden, je nachdem, aus welchen sozialen Gruppen sie kommen, welche Altersgruppe sie repräsentieren oder sogar welcher Partei sie angehören, worauf Kotthoff [2004a] hinweist. Es darf aber gleichzeitig auch nicht vergessen werden, dass das sexuelle Witzeln ein tabuisiertes Thema anspricht, das in vielen Kreisen, unabhängig von der Nation, Kultur und Mentalität, nicht zum Thema der humoristischen Texte werden sollte. Das Durchbrechen von diesem Tabu kann von den Rezipienten verschieden beurteilt werden - das Überraschungseffekt entsteht demzufolge aus der Konfrontation des Verbotenen mit dem Ausgelachten oder Auzulachenden. Das Komische wird dank der Doppeldeutigkeit erzeugt, aber es ist in diesem Falle nicht die semantische Mehrdeutigkeit, weil es in den genannten Szenen eher um das mehrdeutige Verhalten von Francesco geht - wenn er sich neutral zu verhalten versucht, wird er doch, dem Stereotyp nach, eindeutig als ein sexbesessener italienischer Macho verstanden und dann auch so beurteilt.

Wie schon mehrmals betont wurde, haben wir es im Rahmen von „Was guckst du?" mit der Wiedergabe des Multikulturellen zu tun, indem sich der Moderator um eine authentische Darstellung der jeweiligen Figur und der für sie typischen nationalen Identität und Mentalität bemüht. Das Verbale wird geschickt mit verschiedenen Elementen der nonverbalen Kommunikation ergänzt und ergibt somit ein breites Spektrum der witzigen Figuren, Situationen und Sprüche. Eine dieser Witzfiguren, gespielt auch von Kaya Yanar, ist Tarek ab del Kalek, der als Journalist von Dubai TV u.a. die Neuigkeiten von der Modemesse in Düsseldorf darstellen soll. In solchen Fällen wird von den Autoren absichtlich die arabische Sprache gebraucht, die mit der deutschen Sprache konfrontiert wird. Viele Sachen werden in der Originalsprache gedreht, aber die Szenen werden dann mit den Untertiteln versehen, wo der gesprochene Text übersetzt wird und auf diese Weise erfahren wir, ohne Arabisch oder Türkisch zu können, wo der Witz steckt. Manchmal wird auch mit der Kenntnis des Englischen gespielt, wie z.B. in der folgenden Szene: 


\section{(72) Tarek ab del Kalek auf der Modemesse in Düsseldorf 1.}

Tarek ab del Kalek spricht mit einem Model.

T: You speak Deutsch? Allemange, anything you come, spanish? How is it to play in Germania, to make the fashion-show in the..., here?

M: Its good!

$T$ : Is good?

M: Jeah!

$T:$ Is ist different from Spain?

$M$ : I've never modelled in Spain

T: Are you from Spain?

$M:$ No, Kolumbien.

$\mathrm{T}$ : Oh, Kolumbien! How is it to model in Kolumbien?

$M$ : I' ve never modeled in Kolumbien.

$\mathrm{T}$ : Are you model?

$M:$ Yes!

Auch in diesem Fall wird der Mangel an Sprachkenntnissen zum Thema, diesmal geht es um das Englische, wobei die Lexik nur auf einige, von der Figur wiederholte Wörter beschränkt ist.

Als nächstes Mittel des Humoristischen dient die Homophonie, die von den Autoren ziemlich oft benutzt wird, u.a. in einer Szene in dem Gespräch mit dem Vertreter der Firma Kwark festgestellt werden kann. Die saloppe Bedeutung von Quark in der Wendung Quark sein ${ }^{54}$ wird in Bezug auf den Firmnennamen gebraucht, was als Träger des Komischen zu verstehen ist.

\section{(73) Tarek ab del Kalek auf der Modemesse in Düsseldorf 2.}

Tarek ab del Kalek präsentiert die Ausstellung von der Firma Kwark

T: Auf dieser Messe sind Firmen aus der ganzen Welt vertreten. Dahinten Frankreich, hier Italien. Nur Dubai ist nicht (73a) dubei, dabei, duba, duba, du, dabei. T: (73b) Ist das hier alles Quark / Kwark?

${ }^{54}$ Quark, der; -s [spätmhd. quarc, quarg, twarc, aus dem Slaw.; vgl. poln. twaróg]: 1. aus saurer Milch hergestelltes, weißes, breiiges Nahrungsmittel: vollfetter, fettarmer, 40\%iger Q.; Spr getretener Q. wird breit, nicht stark (etw. ohne inhaltliche Tiefe wird auch durch noch so großen Aufwand nicht auf ein höheres Niveau gebracht; Goethe, Westöstlicher Diwan). 2. (salopp abwertend) Unsinn, Unfug, dummes Zeug: so ein Q.!; was soll der Q.?; der Film war ein absoluter Q.; red nicht solchen Q.!; sich über jeden Q. (über jede noch so belanglose Kleinigkeit) aufregen; *einen Q. (ugs.; gar nichts; in keiner Weise): das interessiert mich einen Q.; das geht dich einen Q. an. 2000 Dudenverlag 
Vertreter: Ja, das ist..

T: (73c) Alles Quark. Also Frauen ziehen gern Quark an. (73d) Und für Männer ist es wahrscheinlich Quark, oder?

In den beiden oben angeführten Sketchtexten fallen die fehlenden Englischkenntnisse auf, die zu den Missverständnissen führen, andererseits wird geschickt mit der deutschen Sprache gespielt. In der letzten Szene fällt die Paronomasie (73a) dubei, dabei, duba, duba, du, dabei auf, also das Zusammenstellen lautlich ähnlicher Wörter. Nicht weniger wichtig für die Zwecke der Darstellung der fehlenden sprachlichen Kompetenz bei einem Ausländer ist das Wortspiel mit den Homophonen ${ }^{55}$ und mehrdeutigen Substantiven, z.B. (73b-d) Ist das hier alles Quark / Kwark? (Kwark als Firmenname vs. Quark'1. aus Milch hergestelltes, weißes, breiiges Nahrungsmittel, 2. Unsinn, Unfug, dummes Zeug')

Die letztere genannte Erscheinung ist auch in der nächsten Szene zu sehen, wo die Bedeutungen der Elemente schwarz und (74a) weiß vermischt werden.

\section{(74) Schachweltmeisterschaften.}

Am Tisch sitzen zwei Spieler, wobei der eine schwarz ist.

Richter: Ich begrüße die beiden Spieler im Finale der Schachweltmeisterschaft und eröffne damit offiziell die Partie. (74a) Weiß beginnt

Spieler: He? Wer jetzt?

Das Vermischen der beiden Bedeutungsvarianten der Farbbezeichnungen wegen ihrer Doppeldeutigkeit und das damit zusammenhängende falsche Verstehen des ganzen Kontextes werden zusätzlich mit den außersprachlichen Elementen verstärkt, denn an dem Spiel nehmen ein weißer und ein schwarzer Spieler teil.

Bei der Analyse der Texte aus „Was guckst du?" konnte festgestellt werden, dass vor allem die Elemente der Lexik des Türkischen, des Arabischen und des Italienischen als Fremdwörter im engeren Sinne gebraucht wurden, was mit dem Charakter der Sendung, ihrem Moderator und den Figuren, die von Yanar gespielt wurden, zusammenhängt.

${ }^{55}$ ho $\mid$ mo $\mid$ phon $<$ Adj. $>$ [griech. homóphonos = gleich klingend, übereinstimmend]: 1. (Musik) in der Kompositionsart der Homophonie, wobei die Melodiestimme durch Akkorde gestützt wird u. die Stimmen weitgehend im gleichen Rhythmus verlaufen: eine Komposition in -em Satz, -er Schreibweise. 2. (Sprachw.) (von Wörtern od. Wortsilben) gleich lautend: die vier -en Wortsilben in zweieinhalb Zeilen verletzen... das... Sprachgefühl (Deschner, Talente 334). 2000 Dudenverlag 
Die Bezeichnungsexotismen werden von den Autoren von „Was guckst du?“ bevorzugt, indem in den Texten, die für dieses Programm geschrieben werden, fremdsprachige Elemente erscheinen, u.a. typisch italienische Namen der Speisen Pizza, Lasagne, Spaghetti, Getränke Cappucino oder die der Zubereitungsart al dente oder Bezeichnungen aus der türkischen Küche, z.B. Döner Kebab oder Baklava.

Für die Sendung „Was guckst du?“ ist eine besondere Art Humor charakteristisch, weil die Zuschauer im Studio und die vor dem Fernseher das erzählte Lustige kreativ rezipieren können. Der gesagte Text und das gezeigte Bild werden schnell assoziiert und der Gesamtkontext richtig verstanden, d.h. das Komische wird erzeugt. Die für die Scherzkommunikation charakteristische Mitproduktion weiterer Kontexte liegt auch hier vor, weil sich der Zuschauer oder Hörer weiter darum bemüht, den gehörten witzigen Text richtig zu verstehen. Auf diese Weise tragen verschiedene Assoziationen und die Herstellung der nicht vorauszusehenden Zusammenhänge dazu bei, dass die Scherzkommunikation als ganzer und kreativer Prozess zu verstehen ist. In „Was guckst du?“ überwiegt der konversationelle Humor, wo das Lustige in dem Gespräch versteckt ist und zwischen den Zeilen gehört werden soll. Wenn für den Sachwitz lustige Situationen mit verschiedenen Witzfiguren, zahlreichen Gags und untypischen, unerwarteten, manchmal sogar „unnormalen“ Verhaltensweisen charakteristisch sind, wird im Rahmen der Sprachwitze das den Autoren zur Verfügung stehende breite Spektrum der Sprache auszgenutzt, indem man auf die Polysemie, Homonymie oder Homophonie der deutschen Lexik zurückgreift. Der übertriebene Gebrauch falscher oder nicht ganz korrekter Flexionsformen oder aber die Fehler in der Aussprache werden von den Autoren gezielt eingesetzt - es müssen komische Effekte entstehen und der Rezipient soll zum Schluss lachen. In „,Was guckst du? " hat man es also mit beiden Typen des Witzes zu tun - dem Sachwitz und dem sprachlichen Witz. Nicht weniger wichtig sind natürlich auch die Körpersprache aller von Yanar und seinem Team gespielten Figuren, ihre Kleidung und Verhaltensweise, die der jeweiligen, in einer konkreten Szene und im ganzen Sketch angesprochenen Nation und deren Mentalität entsprechen sollen.

Für das Weiterleben der Sendung sind auch die sog. Sekundärtexte um die Sendung herum wichtig. Yanar gelingt es in verschiedenen zahlreichen Interviews, in denen er sich zu den Funktionen seiner Show äußert und auch die an ihn als Vertreter der Einwanderungsgesellschaft von dem Publikum gestellten Erwartungen anspricht. 


\section{SPRACHLICHE MITTEL DES HUMORISTISCHEN BEI „PARANIENORMALNI“"}

Beim Vergleich der beiden Sprachen im Bereich der scherzhaften Kommunikation fällt auf, dass es auch in der polnischen Medienlandschaft Gruppen gibt, die sich genauso geschickt wie die Textautoren von „Was guckst du?“ der sprachlichen Mittel bedienen, um das Humoristische zu erzielen und demzufolge den Hörer bzw. Zuschauer zum Lachen zu bringen. Das polnische Sprachsystem stellt auch ein großes Inventar von Doppeldeutigkeiten und von polysemen Lexemen zur Verfügung, was man am besten mit den Sketchtexten von der Gruppe „Paranienormalni“" zeigen kann.

Das Komische kann im Falle eines für die Bühne geschriebenen Sketchtextes nicht nur mit den sprachlichen Mitteln erzielt werden, sondern auch in einer geschickten und durchdachten Verbindung des Sprachlichen mit allem Außersprachlichen, was aus dem situativen Kontext, dem Erscheinungsbild der Spielenden und derer Körpersprache resultiert, worauf in dieser Arbeit schon vielmals hingewiesen wurde. Das Vorzeigen konkreter Bilder, verbunden mit einem zu ihnen passenden Text soll im Sinne der Autoren richtig assoziiert und entschlüsselt, dekodiert werden. Erst die beiden Erscheinungen, d.h. die Assoziation und die Dekodierung, sind die Voraussetzung für das richtige Verstehen eines bestimmten humoristischen Textes und gleichzeitig auch aller mit ihm zusammenhängeneden Kontexte. Wir haben es nämlich neben dem Hauptkontext mit den weiteren Kontexten zu tun, die aus der Mitwirkung der Zuhörer bzw. Zuschauer resultieren und gleichzeitig notwending sind, damit der Text richtig interpretiert werden kann. Subjektive Rezeption eines humoristischen Textes bedeutet für den Rezipienten, eigene Assoziationen haben zu können bzw. sogar zu dürfen. Damit hängt noch eine weitere Erscheinung zusammen - das Bilden unvorhergesehener Verbindungen des sprachlichen und des außersprachlichen Kontextes, was wiederum aus der kreativen Rezeption der Realität resultiert. 


\subsection{Sprache und Formate von „Paranienormalni““}

Da in den für die Zwecke dieser Arbeit analysierten Programmen die Form des Dialogs überwiegt, muss wieder betont werden, dass hier der konversationelle Humor an erster Stelle steht. Interessant ist dabei die Tatsache, dass sowohl „Was guckst du?“ als auch „Paranienormalni” diese Art von Humor anbieten. In beiden Sprachen, sowohl im Deutschen als auch im Polnischen, kann auf Folgendes hingewiesen werden - die Polysemie, die Homonymie und die Homophonie sind die meist zum Ausdruck des Komischen gebrauchten sprachlichen Mittel. Interessant ist dabei noch eine weitere Erscheinung, die für "Paranienormalni“" charakteristisch ist, nämlich der übermäßige Gebrauch von Quasivulgarismen ${ }^{56}$, die als Floskeln zu verstehen sind und von Elementen der polnischen Jugendsprache, die für viele Rezipienten nicht verständlich sind (spoksik, narajana, pojechana, no szczera jesteś, wiesz!, rewelka, zarąbisty).

Aus der Analyse der zahlreichen witzigen deutschen und polnischen Texte geht hervor, dass die Art und Weise, wie von den Autoren mit den polysemen und homonymen Lexemen gespielt wird, d.h. welche von den mehreren Bedeutungsvarianten gewählt werden und was darüber entscheidet, eine besondere Bedeutung zugeschrieben bekommen kann. In erster Linie kann bei den polysemen Ausdrücken eine solche Tendenz bemerkt werden, dass von den Autoren in den meisten Fällen die übertragene Bedeutung gebraucht wird, die dem Rezipienten oft entweder nicht bekannt oder nur teilweise bekannt ist. Dasselbe betrifft lexikalische Elemente, die neben ihrer Grundbedeutung auch eine fachliche haben. An dieser Stelle kann man den polnischen Sketchtext nennen (75),, M jak mtotek ", wo zahlreiche Bezeichnungen aus der Handwerkersprache (z.B. wieratrka dostała udaru, francuz kręci śruby, beton wylali, ściana nośna, tapeta odeszła od ściany usw.) benutzt werden, so dass der Text doppeldeutig wirkt und seine Aufgabe als ein humoristischer Text erfüllt.

In einem weiteren Sketch der Gruppe „Paranienormalni“, nämlich „Papieros“ (78), wird von den Autoren und Darstellern geschickt mit der polnischen Sprache und ihren Nuancen gespielt, indem vor allem die Polysemie als sprachliches Mittel des Komischen zu nennen ist. Es werden mit Absicht solche Wendungen gebraucht wie czuć coś przez bibułe 'ein Vorgefühl haben', wypalić się 'keine Kraft und Lust mehr haben, etwas zu tun', rzucać kogoś 'sich von jdm trennen', rozklejać się 'weich werden und in Tränen ausbrechen' oder przyjść cata paczka 'mit der gan-

${ }^{56}$ Unter Quasivulgarismus verstehe ich das polnische Lexem $k u w a$, in dem der Konsonant $r$ weggelassen wird (kuwa vs. kurwa), damit einerseits der Text teilweise „milder“ lautet und seinen obszönen Charakter verliert, andererseits auch insgesamt das Komische in dem jeweiligen Text betont. 
zen Gruppe erscheinen'. In dieser Szene werden sie entweder in ihrer übertragenen Bedeutung gebraucht, oder sie erscheinen in der Grundlesart bzw. ihre beiden Bedeutungsvarianten werden vermischt, so dass noch klarer zu sehen ist, welche Funktion die mehrdeutigen Wörter und Wendungen haben können. Dieser Text wird des weiteren detailliert untersucht und besprochen (S. 119-121).

Mit zahlreichen in den Sketchtexten gebrauchten Lexemen sind in den meisten Fällen mehrere Lesarten verbunden, so dass die fehlende Kenntnis der einen oder anderen Variante zu den Missverständnissen führen kann, z.B.

- drzeć się als 1.'reißen' und 2.'laut schreien',

- odchodzić od 1.'sich von jdm trennen' und 2.'sich von etw. lösen' oder aber

- kleić się 1.'kleben', 2.' sich kleben lassen' und in der übertragenen Bedeutung 3.'in der Beziehung gut miteinander auskommen',

- rozklejać się 1.'sich von etwas ablösen, weil der Klebstoff nicht mehr zusammenhält'2.'weich werden und in Tränen ausbrechen',

- rzucać kogoś/coś 1.'sich von jdm trennen', 2. 'mit etwas aufhören, z.B. mit dem Rauchen, sich etwas abgewöhnen' oder

- paczka als 1.'Schachtel' und 2.'Clique'.

Die zweite, bereits erwähnte sprachliche Erscheinung - Homophonie - hängt, wie es schon vorher betont wurde, damit zusammen, dass zwischen mindestens zwei Lexemen eine phonetische Ähnlichkeit besteht, die nicht nur zur Verwechslung der Lexeme beiträgt, sondern auch die falsche Interpretation impliziert.

Sprachfehler als Normenkonflikte schaffen also günstige Voraussetzungen für Pointenbildungen in Witzen, auch (und gerade) weil sie im alltäglichen sprachlichen Leben nicht so einfach zu behandeln und zu verarbeiten sind [Cherubim 2008, S. 187].

So wie im Falle der Sendung „Was guckst du?“ ist es auch bei „Paranienormalni“ der Fall, dass fremdsprachige Lexik zum Zwecke des Humoristischen eingesetzt wird. In einem Sketch mit dem französischen Tanzlehrer („Tanzunterricht") werden vor allem Bezeichnungen aus dem Französischen gebraucht, ohne dass man sie dann richtig ins Polnische übersetzt. Es erheben sich dabei folgende Fragen:

- inwieweit diese Lexik korrekt ausgesprochen wird,

- ob es tatsächlich Wörter französischer Herkunft sind oder aber

- ob die Darsteller nur so geschickt mit der Sprache spielen, dass man den Eindruck hat, echtes Französisch hören zu können, 
wenn z.B. die polnische vulgäre Imperativform spierdalaj 'Hau ab!' mit dem französischen Akzent ausgesprochen wird. Auch hier spielt man mit den Stereotypen. Die Gruppe bemüht sich darum, so eine Stimmung zu schaffen, die für einen Tanzunterricht mit einem berühmten französischen Lehrer tatsächlich typisch wäre - ein eingebildeter Tanzlehrer, der den Schüler missachtet, ihn ständig provoziert und auslacht, wobei er der Schülerin viel mehr Aufmerksamkeit schenkt und großes Interesse an ihr zeigt. Interessant ist die Tatsache, dass dabei das Publikum mitspielen kann, indem aus ihm eine Frau herausgebeten wird und an dem Sketch teilnimmt. Auch wenn man sprachlich nicht viel verstehen kann, ist der Sketch verständlich - vor allem wegen der Art und Weise der Präsentation und der Körpersprache der Darsteller. Der Lehrer macht langes Gesicht, wenn sein Schüler etwas falsch macht, verpasst ihm sogar Tritte und lacht ihn aus. Der Lehrer ahmt auch den Schüler nach, vergleicht ihn mit einem Schwein, das sich nicht gar nicht leicht bewegen kann oder aber auch mit einem schweren Sumoringer und tut das alles, um dann in Gelächter auszubrechen, was natürlich auch erwartet wird. Dem Franzosen werden dann noch weitere Merkmale zugeschrieben, u.a. sein besonders großes Interesse an Frauen und Sex, was er auf Schritt und Tritt manifestiert. Von Anfang an entsteht eine besondere Verbindung zwischen dem Publikum und der Gruppe, was zum Schluss das Erwartete mitbringt - das Lachen.

In einem anderen Sketch, nämlich „Wesele w chińskiej restauracji“ wird auf die polnische Tradition und die damit verbundenen Sitten und Bräuche angespielt, die einem Ausländer doch nicht bekannt sind, auch wenn dieser eine Hochzeitsparty organisieren soll. Die Darsteller, typisch chinesisch angezogen, erscheinen auf der Bühne und versuchen ihr Bestes zu geben, indem sie auch mit dem chinesischen Akzent sprechen. Da erscheinen in ihrer Sprache u.a. solche Elemente wie statt Boney M. verstanden als bo nie jem, was das Lustige ergibt, denn die Gruppe Boney M. wurde auf der Party erwartet, stattdessen wird ein Chinese vorsingen, weil er während der Party nichts isst. Das geht auf die Homophonie zurück.

Der Vergleich der Texte von „Paranienormalni“ ist zugleich die vergleichende Analyse verschiedener Bühneformen, vor allem die der Sketchtexte in Form eines Dialogs oder eines Monologs. Die Analyse betrifft ausgewählte Texte der Gruppe „Paranienormalni“, und von der Gruppe „Ani Mru Mru“, die von mir für die Zwecke dieser Arbeit verschriftet wurden ${ }^{57}$. Im Vergleich zu den analysierten Sketchen aus „Was guckst du?" werden die in den polnischen Texten gefundenen

${ }^{57}$ Die Gruppe „Paranienormalni“ existiert seit 2004. Am Anfang bestand sie aus Igor Kwiatkowski, Robert Motyka, Rafał Kadłucki (technischer Mitarbeiter), seit 2008 tritt zusammen mit ihnen noch Michał Paszczyk auf. Die Gruppe „Ani Mru-Mru“ existiert seit 1999 und besteht aus Marcin Wójcik, Michał Wójcik und Waldemar Wilkołek. 
sprachlichen Mittel des Humoristischen anders vermerkt, d.h. alle polnischen Elemente in den Sketchtexten der jeweiligen Gruppe werden durchnummeriert.

Aus der Analyse geht eindeutig hervor, dass zahlreiche sprachliche Mittel, wie Wortspiele, übermäßiger Gebrauch von manchen Strukturen oder grammatischen Formen, Wiederholungen, Polysemie, Homonymie und Homophonie, die das Komische bilden sollen, der Ausgangspunkt für die Bildung durchdachter lustiger Texte mit einem unerwarteten Ende, also für die Bildung der Witze sind.

Als Beispiel kann an dieser Stelle der Sketch „M jak młotek“ dienen. Vor allem fällt hier seine Intertextualität auf. Die Autoren berufen sich dabei auf den polnischen Serienfilm „M jak miłość“. Sie ändern jedoch den Titel in „M jak młotek" und den Text des damit zusammenhängenden Liedes. Der Sketch hat die Form eines Dialogs zwischen einem Hammer und einem Nagel. Als Mittel des Humoristischen werden vor allem die neben den fachlichen existierenden übertragenen Bedeutungen bestimmter Lexeme gebraucht, was dazu führt, dass von dem Zuhörer bzw. Zuschauer bei der Rezeption dieses Textes zwei Kontexte mitproduziert werden - der mit der Fachsprache zusammenhängende und der aus der übertragenen Bedeutung resultierende Kontext.

\section{(75) $M$ jak młotek}

Im Hintergrund hören wir die Musik aus dem Film „, M jak miłość“, der Titelsong hat jedoch einen geänderten Text (,,M jak mlotek wiele funkcji ma, M jak mlotek zawsze rade da...). Der Hammer erscheint auf der Bühne und sucht nach dem Nagel, fragt dabei das Publikum, ob jemand ihn gesehen hat.

M: Gwoździu, gwoździu! Słuchajcie, nie widzieliście może gwoździa? Co, nie bardzo? Taki (1) przybity trochę. O, jesteś...

G: O kurcze, czekaj, ale (2) mnie boli szyjka od obrazka. Er bewegt mit dem Kopf und man sieht, dass ihm etwas weh tut.

M: No gdzież ty się podziewał?

G: (3)Tkwiło sie to tu, to tam, nie? A co nowego na budowie?

M: Co na budowie? .4) teb mnie strasznie boli. Er fasst sich am Kopf. Wiesz, słuchaj, jak ja sie wczoraj (5) nawaliłem z kilofem, tak żeśmy (6) się skuli, słuchaj, no, impreza w "Castoramie" była, słuchaj, co mówię, no. Powiem ci, że nawet nie wiedziałem, że (7a) francuz potrafi (7b) takie śruby kręcić, słuchaj, no...

G: Męska impreza, co?

M: Nie była taka męska, wiesz, (8a) podkowa też tam była

$G$ : A ona po co?

M: (8b) Na szczęście, hyhyhy. Nie, no za kierowce robiła, wiesz. 
G: Ty ty ty ty ty ty, a tej, no, wiertarki, nie było z wami?

M: Wiertarki? No co ty! Jaja sobie robisz? Nie słyszałeś o wiertarce? Er ist empört und überrascht, dass der Gesprächspartner von nichts weiß.

G: Nie.

M: (9)Wiertarka na słońcu leżała i udaru dostała. [...]

G: Ty patrz, jakie nieszczęście, co nie? Bo o betonie to styszałeś, prawda?

$M:[. .$.$] No gadaj, co z betonem? Geste und Mimik betonen die Wichtig-$ keit dieser Information.

G: (10) Wylali go!

$M:$ [...] A o tapecie i ścianie styszałeś? [...] (11) Tapeta odeszła od ściany.[...]

(12) Przestało się między nimi kleić i (13) tapeta się zwinęła. G: [...] Przecież (14) ściana zawsze tak była za tapeta.[...]

$M$ : Ale znasz tapete, wiesz, jak (15) ona lubi się drzeć [...] Er zuckt mit den Schultern.

G: (16) A ściany majq uszy! [...] A powiedz, (17a) jak ściana to zniosła?

M: [...] Bardzo dobrze, przecież to (17b) ściana nośna. [...] Trzeba przyznać, że (18a) chociaż beton to(18b) ma kontakty. [...] Mit der Mimik wird die Begeisterung zum Ausdruck gebracht.

In dem oben angeführten Text gibt es vor allem polyseme Strukturen, wobei es vorwiegend Lexeme oder Wortgruppen aus der Handwerkersprache sind. Die von den Autoren geschickt eingesetzten sprachlichen Elemente haben neben der Fachbedeutung auch ihre meist übertragene Bedeutung. Von dem Zuschauer bzw. dem Hörer wird die Kenntnis der Bedeutung der Elemente der präsentierten Texte verlangt, sonst könnten von ihm die weiteren Kontexte nicht identifiziert, dekodiert und im Endeffekt richtig interpretiert werden, z.B. (1) przybity 1.' festgenagelt, 2.'etwas besorgt', (2) mnie boli szyjka od obrazka hat aufgrund der Bedeutung von 1. szyjka Hals als Körperteil und 2.Teil eines Gegenstandes in einer länglichen, halsähnlichen Form' eine vermischte Bedeutung, (3) tkwito się to tu, to tam 1. tkwić 'hier und da sein, 2.stecken', (4) teb 1. 'Kopf', 2. 'der obere Teil eines Gegenstandes',(7a) francuz potrafi, (7b) kręcić śruby, wo wir es mit einem Fachwort francuz als Bezeichnung eines Werkzeuges 'Franzose, verstellbarer Schraubenschlüssel 'zu tun haben und mit der Bedeutung von 1.' Schrauben ziehen und dazu noch 2.' im Sport - Figuren drehen'; (8) podkowa tam była na szczęście 1.' Hufeisen als Symbol des Glücks' und dabei noch 2. 'zum Glück an Ort und Stelle sein'. Interessant ist aber auch die Vermischung der Bedeutungsvarianten in ei- 
nem Satz, wie es im Falle von (9) wiertarka udaru dostata ist, wenn die Konstruktion auf das Gruppenlexem zurückgeht, d.h. udar stoneczny 'Sonnenstich' und auf eine fachliche Bedeutungsvariante von udar in der Bezeichnung eines Werkzeugs wiartarka z udarem 'Schlagbohrmaschine'. Im Falle des Elementes (11) tapeta odeszła od ściany haben wir es mit der Doppeldeutigkeit zu tun, weil das Gesagte unterschiedlich verstanden werden kann - als 1.' sich von der Wand lösen' oder 2. 'sich von jdm trennen', genauso wie es im Falle von (14) ściana była za tapeta ist, wo die Bedeutungen 1.'hinter etwas sein, sich hinter etwas befinden' und 2. 'jdn lieben' vorliegen.

Nicht selten wird von den Autoren auch die Umgangssprache eingesetzt, z.B. (15) tapeta lubi się drzeć, wo die Grundbedeutung von 1.' reißen' in Frage kommt und dazu noch eine zweite Variante, die erst aus dem Kontext entschlüsselt werden soll, d.h. 2'.laut schreien' und (16) ściany maja uszy 1. 'Ohren haben', 2. 'Griffe haben' und daneben eine übertragene Bedeutung 3. 'lauschen'. An einer Stelle in dem angeführten Sketch werden die beiden Bedeutungsvarianten geschickt miteinander kombiniert, so dass die Doppeldeutigkeit entsteht, d.h. (17a) jak ściana to zniosta? 1. 'heruntertragen, 2. etwas durchstehen, ertragen' und (17b) to ściana nośna, wo in (17b) die Grundbedeutung 1.'Tragwand' im Rahmen der Fachsprache im Spiel ist.

Für die Zwecke des Humoristischen werden auch die Elemente der Umgangssprache gebraucht, die neben ihrer Fachbedeutung eben diese umgangssprachliche haben, z.B. (5) nawalitem się als 'sich besaufen' (neben der Fachbedeutung 'schlagen'), (6) żeśmy się skuli 'sich besaufen' (neben der fachlichen Variante 'schmieden, hämmern') (10) [beton] wylali 1.' Beton vergießen' neben der umgangssprachlichen Bedeutung von 2. 'jdn feuern', (12) kleić się 1. 'kleben' neben umgs. 2. 'richtig funktionieren', (13) tapeta się zwinęta 1.'zusammenrollen' und umgs. 2.'wegziehen, weggehen', (18a) chociaż beton 1.'Beton' und 2. 'ein dummer, beschränkter Mensch' to ma (18b) kontakty 1. 'Steckdose', 2. 'Schalter' und die hier gebrauchte Variante 3. 'Kontakt zu anderen Menschen' und im übertragenen Sinne 'Vitamin-B haben, also gute Beziehungen haben'.

Aus der Analyse geht hervor, dass dieser Sketchtext zwei verschiedene Kontexte hat, die dabei gleichzeitig zwei verschiedene sprachliche Kontexte sind, was auf seine fachliche und übertragene Bedeutung zurückgeht, wobei auch der außersprachliche Kontext verschieden, d.h. doppelt verstanden werden kann - als ein Fachgespräch und auch als ein lockeres Gespräch mit den versteckten Hintergrundinformationen, die von dem Zuhörer bzw. Zuschauer entschlüsselt werden sollen. 


\subsection{Quasivulgarismen als Mittel des Humoristischen im Polnischen}

Die Analyse der Sketchtexte hat ergeben, dass den vulgären sprachlichen Elementen im Polnischen eine besondere Funktion im Rahmen des Humoristischen zugeschrieben steht. Eigentlich könnte man wagen zu behaupten, dass es heute kaum gelacht wird, wenn es im Witz keine Vulgarismen ${ }^{58}$ gibt. Wegen der umgangsprachlichen Formen, die in den Witzen auftauchen, ist es der Fall, dass eben der obszöne Wortschatz zum sprachlichen Inventar des jeweiligen Autor der Sketchtexte gehört, was darauf zurückgeht, dass diese Lexik doch einen wichtigen Bestandteil des Gegenwartspolnischen ausmacht.

Bartmiński [2001] weist daruf hin, dass die Umgangssprache einen besonderen Stellenwert innerhalb aller Sprachvarianten des jeweiligen Sprachsystems einnimmt, was in erster Linie darauf zurückzuführen ist, dass eben die Umgangssprache als die erste anzueignende Variante der Muttersprache gelernt wird.

W rzeczywistości styl potoczny obejmuje szeroką gamę wypowiedzi zarówno ustnych z ich wewnętrznym zróżnicowaniem gatunkowym (rozmowy przy stole, na ulicy, w urzędach i sklepach, dyskusje w kolejkach, powitania i pożegnania, prośby i skargi, polecenia i życzenia, gratulacje i kondolencje, groźby i kłótnie, opowiadania wspomnieniowe, relacje ze zdarzeń, flirty, kawały itd.), jak i pisanych, również zróżnicowanych gatunkowo [...]

Styl potoczny nie daje się zamknąć w granicach jednej (np. mówionej) formy przekazu, jednej (np. kolokwialnej) sfery użycia i jednego (np. emocjonalnego) typu postawy. Jest to język obiegowy, powszechny [Ebenda, S. 118].

An dieser Stelle muss aber noch auf eine Erscheinung hingewiesen werden, nämlich auf die Unterscheidung der weiteren Schichten innerhalb der Umgangssprache. Eine der Schichten bilden Vulgarismen, die im Rahmen der Kommunikation einerseits als stark emotional geladene Wörter und andererseits als kurze Unterbrechung verstanden werden, als Wörter, die als notwenige Pausen zwischen den Gesprächseinheiten eingesetzt werden. Der obszöne Wortschatz wird oft in Form von Euphemismen gebraucht, deren Bedeutung keinen so stark emotional geprägten Charakter hat, z.B. wkurzyć się in der „leichteren“Variante als vs. wkurwić się

${ }^{58}$ Dies Element der Lexik wird auch Vulgärausdruck, Element der Fäkalsprache, Gossenausdruck, Gossenwort, [unanständiger] Ausdruck, unanständiges / ordinäres / unfeines / derbes Wort, Kraftausdruck, Kraftwort genannt.

DUDEN gibt folgende Definition an: Vul|ga|ris|mus, der; -, ...men [engl. vulgarism, zu: vulgar= vulgär, ordinär < lat. vulgaris, vulgär] (bes. Sprachw.): vulgäres (1) Wort, vulgäre (1) Wendung. 2000 Dudenverlag 
'sich ärgern, sich aufregen oder Kurza twarz! als 'Scheiße!’ vs. kurwa mać! Diese einerseits weniger stark wirkenden, trotzdem aber immer noch stark emotional markierten Lexeme bekommen im Rahmen des Humoristischen eine ganz neue Aufgabe - die betonen zusätzlich den Charakter der witzigen Aussage, besonders dann, wenn sie in ihren homophonen Varianten gebraucht werden, wie z.B. kuźwa, kurna und kuwa, die anstelle von kurwa gebraucht werden. Ich nenne sie Quasivulgarismen, weil sie eigentlich ihren obszönen Charakter verlieren und keine so stark beleidigende Funktion mehr haben, sondern nur als Floskeln erscheinen.

In einem anderen repräsentativen Text von der Gruppe „Paranienormalni“, nämlich „Mariolka 1“ griffen die Autoren zur Homophonie als Mittel des Humoristischen. Es fällt dabei auf, dass diese Szene die Form eines Monologs hat, der mit einem Telefongespräch zwischen der Hauptfigur - Mariolka - und ihrer Freundin Gabryśka kombiniert ist. Wir hören zwar den Gesprächspartner von Mariolka nicht, aber sie wiederholt kurz und fasst zusammen, was sie gehört hat. Demzufolge kann diese Szene als ein Quasidialog verstanden werden. Die beiden Figuren erscheinen in allen Folgen von „Mariolka “. Die Sprache ist hier eine Mischung aus Elementen der Jugendsprache, Quasivulgarismen, Fremdwörtern im engeren und im weiteren Sinne und aus den mehrdeutigen Lexemen.

\section{(76) Mariolka 1}

Auf der Bühne erscheint der als Frau verkleidete Igor Kwiatkowski, der in diesem Sketch die Figur Mariolka spielt. Mit einer Blondperücke, einen Pullover in grellen Farben anhabend, mit einer kleinen umgehängten Tasche. Wenn die Gestalt auf der Bühne erscheint, hören wir die Musik von Danzel (,,Don 't you know, pump it up, You got to pump it up, Don 't you know, pump it up, You got to pump it up").Dieses Musikmotiv begleitet Mariolka in jedem Sketch und wird somit zum Wiedererkennungszeichen dieser Figur.

Słuchajcie, (19) ja pitole, (20) jestem taka podjarana, taka podenerwowana, że szok, normalnie, (21) kuwa, tipsy zjadłam, no mówię wam, (22) tera mi sie plastikiem odbija. Mam takiego (23) newsa dla Gabryśki, że jak jej powiem, to (24) kuwa, padnie. Greift zum Handy und versucht Gabryśka zu erreichen. Super, (25) kuwa, granie na czekanie! Man hört die Musik „Don't you know, pump it up, You got to pump it up, Don't you know, pump it up, You got to pump it up" No cześć, Gabryśka, Mariola mówi. A ty kto (26) kuwa, jesteś, a kim pan jest? Ale ja (27) kuwa nic nie rozumiem po polsku, jaki (28) „the subscriber you hale called” " [...] (29) Kuwa, co to było? (30) Kuwa, ale (31) jestem pojechana. Sie versucht 
noch einmal Gabryśka zu erreichen. No cześć, no! Teraz dobrze, Gabryśka, słuchaj, mam dla ciebie takiego (32) newsa, że padniesz, dziewczyno, Mariolka ist nervös und läuft auf der Bühne hin und her (33) ja pitole, stoisz, to (34) kuwa usiadź, siedzisz, to (35) kuwa wstań, Gabryśka, twoja przyjaciółka (36) sensu stricto, czyli ja, (37) kuwa, wygrałam zagraniczna wycieczke, słuchaj, no gdzie jedziemy, gdzie się (38) kuwa bawimy, no nieważne, taki (39a) casting_był „Zostań twarza firmy budowlanej " i (40) kuwa wygrałam. (39b)Co będe reklamować? Pustaki! No, (41) spoksik, spoksik. Gabryśka, no to gdzie się bawimy, gdzie szalejemy [...] Gabryśka, (42) gdzie jedziemy, gdzie się (43) kuwa bawimy? No, ja słyszałam, że jak sie jedzie na zagraniczna wycieczke, to się koniecznie trzeba (44) sczepić. No, ja wiem, że głupio, ty będziesz chciała gdzieś pójść, to jak pójdziemy takie sczepione? No, no. Mit der Mimik und den Gesten gibt sie ein Zeichen, dass sie derselben Meinung ist. Co? (45a) Paszport mam, a po co mam go (45b) przedłużać? Żeby mi się do torebki nie zmieścił? Greift zu ihrer Tasche und zeigt, wie groß diese ist. Ty, zabiore ten krem, co mi dałaś. No dziewczyno, (46) rewelka, (47) zarabisty jest, ale mi pudełka nie dałaś, nie wiem, na co on jest, co on robi. No, weź mnie (48) kuwa nie strasz, (49) ściaga pory??? Mit Angst greift sie an ihre Hose. Szczera jesteś, wiesz? Dobra, Gabryśka, [...] (50) gdzie jedziemy, gdzie się (51) kuwa bawimy? Co? (52a) Morze Egejskie? No wariatko, (52b) kuwa zwariowałaś? Dwa tygodnie z (52c) egejami? (54) Hello! Sie ist empört. Man sieht es ihr an. Ty, (55a) kuwa., gdzie ty, zaraz mnie, (55b) kuwa ${ }_{\Perp}$ na wyspe Lesbos wyślesz! I co byśmy tam w ogóle robiły? Skoki na (56) bungee? Super. A ty wariatko, (57) kuwa widziałaś, jakie to małe jest, no ja widziałam gościa w telewizji, jak na tym (58) bungee grat, no! Ahmt einen Banjospieler nach. Torebke (59) se kupiłam, (60) podrabiana imitacja sztucznej skóry. Słuchaj, okazyjnie, w tym sklepie "Wszystko po 5 złotych". Jak to (61) kuwa, ile dałam? Gabryśka, (62) kuwa, sklep po 5 złotych. (63)Don't matter! [...] A tak w ogóle, to co byśmy tam [na Hawajach] robiły? W czym spały? $W(64)$ bunga... bungalowach? A coś ty, ja tam tradycyjnie, jak ciepło jest, w samych gaciach wolę. A w ogóle słyszałam, że na Hawaje się (65) zarabiście długo pociagiem jedzie. Samolotem to (66) kuwa_zapomnij, zapomnij, wiesz, jak ja (67) kuwa gadać lubie, a słyszałam, że tam na lotnisku jest taka (68) strefa bez słowa. Strefa bez słowa, mówię ci. [...] Dobra, Gabryśka, dawaj, gdzie jedziemy? Jamajka odpada, nie, nie, nie, tam sa te (69) laleczki łubudu, to odpada. [...] Ciebie jak o coś 
poprosić, to tak jak z tym calgonem, tak żeś mi poradziła. Co z (70) calgonem? Jeszcze się (70a) kuwa pytasz, beznadzieja! Nie dość, że (70b) kamienia nie usuwa, to wiesz, (70c) kuwa, jaka zgage miałam!

In dem genannten Sketch sind am meisten die Elemente der polnischen Jugendsprache zu sehen, d.h. (19) ja pitolę 'nicht schlecht, Scheiße!', (20) jestem taka podjarana 'ich bin so begeistert', (31) jestem pojechana 'ich bin so verrückt',(41) spoksik, spoksik 'immer mit der Ruhe', (46) rewelka 'toll, cool', (47) zarąbisty 'unglaublich, toll, phantastisch' und (65) zarabiście 'niesamowicie'. Daneben haben die Autoren auch die Quasivulgarismen benutzt, als sich ständig wiederholende Floskel wird das Wort kuwa gebraucht (21, 24, 25, 26, 27, 29, 30, $34,35,37,38,40,43,48,51,52 \mathrm{~b}, 55 \mathrm{a}-\mathrm{b}, 57,61,62,66,67,70$ a,c).

Außerdem werden hier die doppeldeutigen Wörter gebraucht, wie z.B. (44) przedłużać paszport 'Gültigkeit des Passes verlängern' vs. przedtużać coś 'etwas verlängern', (48) ściagać pory im Sinne von 'Hautporen verkleinern und unsichtbarer machen' vs. ściagać pory 'Hose runterziehen', (70b) kamienia nie usuwa im Sinne von 'Zahnstein entfernen' vs. 'Kalk entfernen'. Wichtig ist, dass es dabei noch um eine bekannte Marke Antikalk-Mittel für Waschmaschinen „Calgon“ geht. Die polnischen Bedeutungen von kamień 1.'Zahnstein' und 2.'Kalk' werden vermischt oder eher absichtlich verwechselt, was einen komischen sprachlichen und situativen Kontext ergibt.

Eine weitere interessante Erscheinung ist der Gebrauch der Anglizismen und die Kritik der fehlenden Englischkenntnisse, d.h. Englisch wird entweder gar nicht (27) the subscriber you have called oder falsch verstanden (56) skoki na bungee 'Bungeejumping', was von Mariolka als *'Banjojumping'verstanden wird, (58) na tym *bungee grat statt banjo, (63) don't matter statt it doesn't matter, (64) spać $w$ *bungalowach, wo bungalow in diesem Kontext als Bezeichnung der Nachtwäsche funktioniert. Daneben wird auch das fehlende Grundwissen kritisiert und ausgelacht, was sich im falschen Verständnis mehrerer Bezeichnungen manifestiert, z.B. es gibt homophone Formpaare der gewählten Strukturen, wie strefa bez stowa (67) 'ruhige Zone' anstatt strefa bezclowa 'zollfreie Zone', genauso wie es im Falle von laleczki łubudu (69)'*łubudu Puppen' anstatt laleczki woodoo 'Woodoo Puppen' oder sczepić się (43)'aneinanderklemmen' anstatt szczepić się 'sich impfen lassen' ist. Es werden auch falsch verstandene bzw. der Figur nicht bekannte Wörter gebraucht, wobei das Komische eben aus dem fehlenden Grundwissen resultiert, d.h. mit den folgenden Bezeichnungen (52a) Morze Egejskie, (52c) egejami werden homosexuelle Männer und Frauen (55b) na wyspę Lesbos assoziiert. In den ersten beiden Fällen haben wir es mit der Verwechslung zu tun, 
d.h. Egejskie wird als 'mit den Homosexuellen zusammenhängend, sie betreffend' verstanden wegen des polnischen Wortes gej 'homosexueller Mann', das der Figur fälschlicherweise als * egej bekannt ist.

Im zitierten Sketchtext wurden auch falsche Wortformen gebraucht, was den Hörer bzw. Zuschauer zum Lachen bringen soll, d.h. (21) *tera statt teraz 'jetzt', $(41,49) *$ gdzie jedziemy statt *dokąd jedziemy, (59) *se kupiłam statt sobie kupiłam und das Anhäufung von Adjektiven in der attributiven Funktion: (60) podrabiana, sztucznej zusammen mit dem synonymen Lexem (imitacja skóry) zu der ganzen Wortgruppe, was noch deren Bedeutung betont - (60) podrabiana imitacja sztucznej skóry.

Die Autoren bedienen sich auch der fremdsprachigen Elemente, deren Bedeutung unserer Mariolka-Figur doch bekannt ist, d.h. aus dem Englischen (22, 31) mam newsa 'ich habe Neuigkeiten' und aus dem Latein (35) sensu stricto.

Nicht weniger wichtig ist das Spiel nicht nur mit der Sprache, sondern auch mit den Assoziationen, was im Falle der Aussage (39a-b) zu sehen ist, wo sich die Autoren auf das Vorwissen der Zuschauer und auf die Assoziationen mit den Blondinenwitzen berufen, wobei die umgangssprachliche Variante von pustak 'dumme Frau' erwartet wird (39a) casting byt „Zostań twarza firmy budowlanej” i (40) kuwa wygrałam. (39b) Co będę reklamować? Pustaki!

Wegen der Zugehörigkeit zu demselben Wortfeld 'Bauarbeit' hängt die Grundbedeutung von pustak 'Lochziegel' mit der von firma budowlana 'Baufirma' zusammen.

Auch im nächsten Sketch, wo Mariolka als Hauptfigur auf der Bühne erscheint, fällt es auf, dass man für die Zwecke dieser Texte bestimmte sprachliche Erscheinungen geschickt ausnutzt, indem man mit der polnischen Lexik und ihrem manchmal fehlendem Verständnis spielt. Die Form ist gleich, d.h. Mariolka führt wieder ein Quasigespräch mit ihrer Freundin Gabryśka und unterhält sich mit ihr darüber, was sie neulich erlebt hat, erinnert sich an ihre Arbeit als Hostess in einem Supermarkt, wo sie den Kunden das Katzen- und Hundefutter als Kostprobe angeboten hat, wobei ihre Freundin noch schlechter war, denn sie hat den Kunden die Reinigungsmilch zum Probieren empfohlen. Mariolka erzählt von ihrem neu kennen gelernten Freund und dem „McDonalds-Test“, dem sie ihn unterziehen will, um zu prüfen, ob sie beide irgendwelche Zukunft haben. 


\section{(77) Mariolka 2}

Auf der Bühne erscheint wieder der als Frau verkleidete Igor Kwiatkowski und wir hören die Musik von Danzel (,,Don't you know, pump it up, You got to pump it up, Don't you know, pump it up, You got to pump it up")

(71) Hejka! Cześć! Czekajcie, czekaj, telefon mam! Poczekajcie, Gabryśka dzwoni, poczekajcie... Gabryśka cześć! Hej Gabrycha. Gabryśka, skad Ty (72) kuwa wiedziałaś, że ja tu bede ? Nieważne. Gabryśka, czekaj, Gabryśka! Ty masz dzisiaj urodziny, (73) co nie? No to Gabryśka! (74) Happy Birthday kuwa to you! Happy Birthday kuwa to you! (75) Brechtasz się? No ja też! Sie lacht. Co robisz? (76) Bez kitu! Uczysz się? Ja (77) pitole! Naprawdę? Gabryśka, to ja słyszałam, że jak się człowiek dużo uczy, to musi dużo (78) magnezu jeść, masz magnez? Jak nie masz, widziałam, na lodówce masz, no! Sie lacht. Tylko tego plastiku (79) kuwa nie jedz, nie? A słuchaj, (80) a propo w ogóle nauki na uczelni, to muszę ci się pochwalić, że (81) założyłam kółko na uczelni, wiesz? No, metalowe, na głowe, (82) kuwa ściagnać nie mogłam! No mówię ci! Nie wiem, sylwestra tu maja czy coś, szaleja nie... Ale w ogóle, Gabryśka, smutna mam nowine, słuchaj, nie jestem już hostessa w supermarkecie, wiesz... Dlaczego, dlaczego, nie wiem dlaczego, wiesz... Byłam na spożywczym, swoja osoba jedzenie reklamowałam, pomyślałam (83) se wiesz, nie wyłożę na tacę, ludzie nie spróbuja, (84) kuwa nie kupia co nie? Ty, wyłożyłam (85) zarabiście wszystkim smakowało, Ty, nie? Przyszła kierowniczka, zobaczyła to, z gęba na mnie, wywaliła mnie, wiesz? A (86) zarabiste jedzenie w takich fajnych puszkach z pieskiem i kotkiem, no mówię ci, no. Sie lacht. A co, co, co, ty nie lepsza byłaś! Nie pamiętasz za co ciebie wywalili? No za to mleko, (87) helot, no... No jakie mleko, nie pamiętasz, co wszystkich mlekiem częstowałaś, a to się okazało, że to mleczko do demakijażu było! Sie lacht. (88) Ja pitole, ja nigdy (89) tyle pawi na raz nie widziałam, normalnie szok... Sie lacht. Gabryśka, ale się w ogóle tym nie przejmuje, wiesz. Mam nowa robote, wiesz? W solarium (90) bede robiła, mówie ci. (91) Zarabiste, takie dużo, yyy imienia yyy... Andrzeja Leppera! Sie lacht. Gabryśka, (92) bez kitu, ty nie wiesz kto to jest Lepper? (93) Ja pitole, (94) jaka siara, dziewczyna...! (95) Kuwa, ja nie moge, nie wiesz, kto to jest Lepper? Gabryśka, to piłkarz jest, co ty! Ale słuchaj ty, poszłam do tego szefa na (96) rozmowe kwa... kwa... kwalifikacyjna, o! Ty i go pytam, (97) ile miesięcznie można wyciagnać, a on mówi, że 1500, myślę (98) spoko 
pieniadz, co nie? To się go pytam, kiedy moge przyjść, a on mówi (99) „powiem pani ten sposób - w ogóle tu pani nie musi przychodzić"! (100) Czujesz to?! (101) Bede miała 1500, w ogóle do roboty nie muszę przychodzić! A Gabrycha! Gabrycha, mam nowego chłopaka. (102) Zarabisty, (103) rewelacja, niebieskooki blondyn, dociera to (104) kuwa do ciebie? (105) Czujesz to? (106) Rewelacja, słuchaj, przystojny, namiętny... Jak ma na imię? Gabryśka, (107) heloł, heloł! Przecież ja mam jakieś zasady, na pierwszej randce o takie rzeczy nie pytam, co nie? Co ty... Tylko żeśmy się całowali, wiesz. Ale słuchaj, zabiera mnie na romantyczna kolacje... do McDonald'a! Sie lacht. O (108) kuwa Gabryśka, (109) o kuwa, Gabryśka ty, (110) co to takiego jest przypomnij mi, ty, jak boli głowa i obrazki sa? Jak boli głowa i obrazki sa co to jest? Pomysł, właśnie, pomysł mam, ty! Słuchaj no, zrobię mu ten nasz test, pamiętasz? Jak kupi (111) cheeseburgera to sknera, co nie? Jak cały zestaw, znaczy, że ma gest, nie? Jak (112) Happy Meal, znaczy, że rodzinny jest, dzieci lubi, wiadomo (113) o co kaman, (114) co nie... Ty, a jak (115) wieśmaka, to wiadomo! Kupuje wieśmaka - zmieniam chłopaka, nie! Sie lacht. A Gabryśka... Gabryśka, pamiętasz tego pieprzyka co go miałam? No (116) co na niego tak chłopaki leciały... No co mnie z ta wokalistka mylili. Jak która ta co śpiewa to "Nieee Heee". Kojarzysz? No Gabryśka! "Nieee Heee”. (117) Kuwa to czekaj, to ci obszerniejszy fragment "Mam dość, nie mów mi nic, pozwól żyć, pozwól mi byyć, nie chce już..."No, słuchaj, to ja już nie mam tego pieprzyka... Taa, usunełam... Sam zszedł, bo to kleszcz był! No wiem, ja też (118) sie nie brechtam, no... A jeszcze ci nie mówiłam, ze mandat ostatnio dostałam, wiesz! No wiem, że (119) prawka nie mam, ale posłuchaj jak było. Ty, (120) jechałam na balety, na stopa, wiesz, (121) zatrzymałam się na wylotówce i (122) kuwa gibie przy trasie, co nie? Ty, ale się nikt nie chce zatrzymać, (123a) co nie, (123b) w końcu się zatrzymat koleś, ale tirem, (124) co nie! Ty, on wysiada i się mnie pyta, (125) czy mu nie pomoge towaru rozładować! (126) Wiadomo o co kaman, (127) co nie? Ty, więc mu mówie (128) no sex, ty, a on za mna, ty, ja biegne, a on za mna, Gabryśka, tak (129) spieprzałam, że mi fotoradar zdjęcie zrobił! No, mówię ci! A Gabrycha, Gabryśka, a gdzie ty w ogóle jesteś (130) pojechana na maxiora ty krejzolko jedna, co? W kuchni? Gabrycha koniecznie idź to dużego pokoju. Do dużego pokoju idź i mi drzwi od balkonu otwórz, bo się (131) kuwa zatrzasnęłam 
Wieder erscheinen hier übertrieben oft gebrauchte Quasivulgarismen kuwa $(72,79,82,84,95,104,108,109,117,122,130)$, ein Vulgarismus (129) spieprzatam 'ich bin so schnell weggelaufen', zahlreiche Elemente der polnischen Jugendsprache und der Umgangssprache, d.h. (71) Hejka! 'Hallo!', (77, 88, 93)*ja pitole 'nicht schlecht, Scheiße!', $(85,86,91,102)$ zarąiście /zarabisty/ zarabiste und (106) rewelacja als 'super, toll!', (94) jaka siara 'was für Schande!', (130) pojechana na maxiora ty krejzolko als 'Du bist so verrückt!', (89) tyle pawi na raz nie widziałam 'ich habe nie so viele auf einmal kotzende Menschen gesehen', (92) bez kitu 'echt!', (95) Kuwa, ja nie mogę! Im Sinne von 'Mann, das trage ich nicht mehr aus!', (98) spoko pieniądz 'ganz schön viel Geld!',(118) się nie brechtam 'ich lache nicht', (120) jechatam na balety, na stopa 'Ich war unterwegs zur Party, fuhr per Anhalter', (122) kuwa gibie, (125) czy mu nie pomogę towaru rozładować 'ob ich Sex mit ihm will?' .

Unter den Elementen der Jugend- bzw. Umgangssprache gibt es auch Wörter fremder Herkunft, die oft entweder nicht korrekt ausgesprochen oder sogar falsch verstanden werden $(87,107)$ helot 'Hör auf, erzähl keinen Unsinn!', (80) *a propo statt a propos, (125) no sex, (126) Wiadomo o co kaman! 'Man weiß, worum es geht'. Daneben erscheinen in diesem Text auch Eigennamen, die als Bezeichnungen der von McDonalds angebotenen Sachen zu verstehen sind, wie (111) cheeseburger, (112) Happy Meal und (115) wieśmak. Als Fremdwörter im engeren Sinne sind dann (74) Happy Birthday und (125) no sex zu verstehen, die in ihrer Originalform gelassen werden.

In der Sprache der Mariolka-Figur wimmelt es von Floskeln ${ }^{59}$, die als Unterbrechungselemente gelten, wie z.B. der schon erwähnte Quasivulgarismus kuwa $(72,79,82,84,95,104,108,109,117,122,131)$, co nie? 'nicht wahr?'(73, 111, $114,122,127)$, das genannte Element $(87,107)$ helot und $(100,105)$ Czujesz to?! 'verstehst du das?'.

In diesem Sketch werden auch die mit dem Autofahren zusammenhängenden Wörter gebraucht, wie z.B. (119) prawka nie mam 'ich habe keinen Führerschein', (120) jechatam na balety, na stopa 'Ich war unterwegs zur Party, fuhr per Anhalter' , (121) zatrzymałam się na wylotówce 'Ich blieb an der Ausfallstraße stehen', (122) gibie przy trasie 'Ich bleibe an der Straße stehen', (123b) w końcu się zatrzymat koleś, ale tirem 'Endlich hielt jemand an, aber in einem LKW'.

Auch hier wird das Humoristische mit der Homophonie erzeugt, d.h. (78) magnez 'Magnesium' wird mit magnes 'Magnet' verwechselt, wobei das zweite

${ }^{59}$ Flos $\mid$ kel, die; -, -n [lat. flosculus= Blümchen, eigtl.= Redeblume, Vkl. von: flos, 1Flor]: nichts sagende Redensart; formelhafte, leere Redewendung: eine höfliche, abgedroschene, eingefahrene F.; seine Rede bestand nur aus -n. 2000 Dudenverlag 
Wort und seine Bedeutung eher von den Rezipienten aus dem Kontext entschlüsselt werden sollen, denn Mariolka sagt ihrer Freundin, dass bei dieser zu Hause so was an der Kühlschranktür hängt. Auch die Wendung założyć kótko wird von der Figur im wortwörtlichen Sinne gebraucht 'sich einen Ring um den Hals herum anlegen', aber zuerst von dem Publikum und - wie es sich später herausstellt - auch von ihrer Gesprächspartnerin eher als 'Interessenzirkel gründen' verstanden, was im Ausbruch des Gelächters manifestiert wird.

In den Sketchtexten erscheinen auch ganze Sätze mit dem versteckten komischen Inhalt, wie z.B. das schon erwähnte Beispiel (89) tyle pawi na raz nie widziałam, normalnie szok und der Satz (81) założyłam kótko na uczelni bzw. (97) ile miesięcznie można wyciagnać 'Wie viel kommt dabei für mich heraus?'.

Unter den Sätzen gibt es aber auch solche, die das fehlende Grundwissen der Hauptfigur auslachen und ihre Klugheit in Frage stellen (99) ,powiem pani ten sposób - w ogóle tu pani nie musi przychodzić"! 'Ich sage es Ihnen so, dass Sie gar nicht kommen brauchen' (dieser Satz wird von Mariolka falsch verstanden als Angebot einer Arbeit, bei der man gar nicht arbeiten braucht) und (110) co to takiego jest, przypomnij mi, ty, jak boli głowa i obrazki sa?? 'Sag mal, was ist das, wenn man Kopfschmerzen hat und Bilder sieht?', der so und nicht anders formuliert wird, weil Mariolka sich nicht mehr an das polnische Wort pomyst 'Idee' erinnern kann.

Im Sketch gibt es auch mit Absicht eingesetzte Fehler, wie z.B. (80) * a propo statt a propos, $(83) *$ se,$(87,107) *$ helot $(90) *$ bede robiła $(72,101) *$ Bede miała, (116) co na niego tak chtopaki* leciaty... und die schon früher genannten Wörter und Wendungen bzw. Sätze $(77,88,93)^{*}$ ja pitole 'nicht schlecht, Scheiße!' *kuwa $(72,79,82,84,95,104,108,109,117,122,131)$.

Kleine Probleme mit der Aussprache der schwierigen Wörter werden auch hier präsentiert (96) rozmowę kwa... kwa... kwalifikacyjng

Es muss noch darauf hingewiesen werden, dass sowohl von dem Zuschauer als auch von dem Zuhörer, also unabhängig von der nonverbalen Ebene, das Grundwissen aus verschiedenen Bereichen verlangt wird, damit der Witz zur Geltung kommen kann, indem die Rezipienten nicht nur den sprachlichen Kontext richtig verstehen können, sondern sich auch auf ihr kulturelles, politisches, technisches usw. Wissen berufen und somit die Verbindung zwischen dem Gesagten und der außersprachlichen Realität herstellen können. Nicht weniger wichtig ist dabei auch die Tatsache, wie man sich als Zuschauer die Freundin von Mariolka vorstellt, denn das sich daraus ergebende Bild lässt in jedem Falle eine andere Interpretation zu, je nachdem, wie wir als Zuschauer den weiteren situativen 
Kontext bilden, d.h. je nachdem, welches Bild von Gabryśka im Bewusstsein der Rezipienten entsteht, das dann noch die Möglichkeit gibt, die beiden Figuren zu vergleichen, denn nur auf diese Weise kann man sich ein volles Bild von dem Außersprachlichen schaffen, das zusammen mit der Sprache der genannten Texte eine Einheit bildet und bestimmte Interpretationen zulässt. Der sprachliche Kontext deutet jedoch darauf hin, wie intelligent diese zweite Figur sein soll, weil die durch verschiedene Fehler gekennzeichneten Aussagen selbst schon unsere Vorstellung von dieser Frau beeinflussen. Die beiden Figuren bilden eine gemeinsame Zielscheibe - die Frau, zusätzlich noch wird Mariolka mit den Blondinenwitzen assoziiert und eben als Figur dieser Art Witze dumm, naiv, wenig wissend, aber gleichzeitig selbstbewusst ist, was ihr eigen- und einzigartiges Auftreten definiert und es auch zum Markenzeichen der ganzen Gruppe macht. Im Falle der Mariolka-Sketche haben wir es damit zu tun, dass die Zielscheibe, das Thema und das Motiv gleich sind - es geht dabei um eine Frau.

In dem nächsten Sketch „Papieros“ wird auch gezielt mit der Doppeldeutigkeit der polnischen Lexik gespielt. Der auf der Bühne stehende Mann erzählt seine Geschichte, d.h. welche Schwierigkeiten ihm das Rauchen bereitet und dass es ihm schwerfällt, mit dem Rauchen aufzuhören. Plötzlich erscheint auf der Bühne sein langjähriger Freund - die Zigarette und es beginnt ein Gespräch, wo sich die Zigarette darüber beschwert, dass sie von unserem Helden verlassen wurde und wie es dazu kommen konnte. Der Rezipient wird mit der bildhaften Darstellung einer ziemlich komplizierten Beziehung konfrontiert, wo das Außersprachliche, das schon an sich außergewöhnlich ist (der Mensch unterhält sich mit einer Zigarette) mit dem Sprachlichen noch stärker betont wird und die Verbindung der beiden Kontexte bringt auf diese Weise den Rezipienten zum Lachen.

\section{(78) Papieros}

Wir sehen auf der Bühne einen Darsteller ( $R$ - Robert), der das Publikum nach Zigaretten fragt. Gleich erscheint auf der Bühne eine Zigarette (in dieser Rolle sehen wir Igor Kwiatkowski).

R: Słuchajcie, ma ktoś może papierosa?Albo nie... Słuchajcie, chodzi o to, że ja teraz jestem $w$ takiej sytuacji obecnie, że mi ciężko o tym mówić, ponieważ ja palę i próbuję rzucić, ale mi się nie udaje, wiecie? I to jest tak z nałogiem, że ja sobie zdaje sprawe, że to jest szkodliwe i...Na przykład dzisiaj rano wstałem z taka myśle, że ani jednego więcej, słuchajcie, ani jednego więcej papierosa. Takie miałem postanowienie i nie da rady! Normalnie od rana (132) chodzi za mna papieros. Słuchajcie, ta myśl mi się pojawia i od razu... 
Auf der Bühne erscheint die Zigarette, gespielt von Igor Kwiatkowski

I: A kuku, cześć! Haha! Hej, hej!

R: A co ty tu robisz?

I: Co ja tu robię? No nic, wiesz, nie myśl, że cię śledzę czy coś. Po prostu zupełnie przypadkowo sobie tędy przechodze, wiesz, koło Rotundy, patrze, stoisz... podchodze, nie?

Zigarette lacht, dann fängt sie an zu weinen

R: Co się dzieje? I: Jak co się dzieje, nie udawaj! Robert, (133) ty mnie ostatnio unikasz!

R: Nie no, coś ty...

I: Co nie, nie kłam! Tylko mi tu nie kłam! Robert, ja (134) czuje przez bibułe, że coś się święci. Co się dzieje?

R: Oj, bo (135) ja się wypaliłem chyba!

I: Wy... (136) Wypaliłem...? Ty draniu, ty... (137) Najpierw mnie podjarałeś...

R: Nie no, co ty mówisz...?

I: (138) A teraz rzucasz, jak pierwszego lepszego kiepa...! Ty draniu, przyznaj się,(139) że chciałeś mnie tylko wykorzystać! Że chodziło ci, jak każdemu, tylko o nikotynę! R: Słuchaj... niee!

I: Nie? Robert, no...

R: $O j$, bo to (140) matka mi kazała cię rzucić!

I: Oooo... Mamunia! Cholerne babsko... Nigdy mnie nie akceptowała! Musieliśmy się chować po kiblach jak gówniarze! A pamiętasz nasza wspólna piosenkę, pamiętasz, no? Idzie rak, nieborak...

I und $R$ singen zusammen: ...masz na płucach czarny znak.

I: Ej, pamiętałeś ty kokiecie, pamiętałeś!

R: Ale tak było kiedyś!

I: Co kiedyś...

R: Teraz sobie jakoś radze, mam gumy z nikotyna!

I: Pff... Gumy z nikotyna... Weź mnie nie rozśmieszaj. (141) Nędzne substytutki! No Robert, no! (142) Masz mnie tu jak na tacy i nic, i nic ?! No, Robert (143) rozpal mnie! Bo nie wytrzymam... Mam zapałki... No? No?!

R: No ja już nie wiem...

I: Nie wiem... Może ci mamunia powie?!

R: Ale nie płacz teraz!

I: Co nie płacz...

R: No (144) nie rozklejaj się, bo ci się tytoń wysypie, no...

I: (145) To puść dymka, badź mężczyzna!

R: Dobra, po kolacji! Tylko ani słowa matce!

I: (146a) Ha, twój pecik pragnie twoich (146b) usteczek! 


\section{R: Po kolacji mówię! \\ I: Dobra, dobra, dobra, znam cię, na jednym się nie skończy, (147) przyjdziemy cała paczka, ha!}

Der ganze Sketchtext ist doppeldeutig. Die Autoren bedienen sich der gleichwertigen Kontexte - des sprachlichen und des situativen. Der eine nur wäre ohne den anderen weniger verständlich. Um das Komische zu erzeugen greift man zur polysemen Lexik und denkt eine mehrdeutige Situation aus, in der sich beide am Sketch teilnehmenden Figuren befinden, was an eine Trennung innerhalb einer bisher gut funktionierenden Beziehung erinnert. Ein nicht zu lösendes Problem der Raucher - vergebliches Versuchen, mit dem Rauchen aufzuhören, wird auf der Bühne in Form eines Dialogs zwischen einem Raucher und einer Zigarette präsentiert. Im Vergleich zu den Mariolka-Sketchen ist dieser viel kürzer, dafür aber haben wir als Zuschauer die Möglichkeit, beide Gesprächspartner zu sehen, ihre nonverbale Sprache zu beurteilen und somit das Sprachliche mit dem Außersprachlichen so wahrzunehmen, dass wir im Endeffekt lachen.

Der Text fängt mit einer im Polnischen ziemlich geläufigen Konstruktion chodzić za kimś im übertragenen Sinne 'von jdm erträumt sein, begehrt, ersehnt sein' an und mit dem Satz (132) chodzi za mna papieros 'ich träume jetzt von einer Zigarette, ich würde jetzt gerne eine Zigarette rauchen' werden wir in die Problematik des Sketches eingeführt, des Weiteren erscheint im Text die Aussage (144) nie rozklejaj się, bo ci się tytoń wysypie, in der auch nicht nur auf die Grundbedeutung von rozklejać się 1.'nicht festhalten, auseinandergehen', sondern auch gleichzeitig auf die übertragene Variante gezeigt wird 'weich werden, gerührt sein, in Tränen ausbrechen'.

Im oben zitierten Text fällt der emotionale Charakter auf, der mit Hilfe der Diminuierung $^{60}$ (146a) Ha, twój pecik pragnie (146b) twoich usteczek! zum Ausdruck gebracht wird, wobei (146a) Ha, twój pecik als 'deine kleine liebe Kippe' und (146b) im Sinne von twoje usteczka 'deine lieben Lippchen, dein liebster Mund' starke Gefühle der Zigarette dem Raucher gegenüber ausdrücken. Mit (141) Nędzne substytutki! wird von der Zigarette die Kaugummi als Nicotinersatzprodukt in Frage gestellt, was im Wort substytutki zu sehen ist, das mit Hilfe der Wortbildung, analogisch zu prostytutki 'Prostituierte' gebildet wird. Die Verbindung dieser Bezeichnung mit dem Attribut nędzne 'miserabel, erbärmlich' hat auch einen starken emotionalen Charakter.

${ }^{60}$ Di|mi|nu|tiv, (auch:) Deminutiv, das; -s, -e (Sprachw.): eine Verkleinerung eines Substantivs ausdrückende Ableitung; Verkleinerungsform (z.B. Öfchen, Gärtlein). 2000 Dudenverlag 
Im Satz (133) Robert, ty mnie ostatnio unikasz! wird die Bedeutungsvariante von unikać czegoś 'etwas meiden' mit der von unikać kogoś 'sich von jdm fernhalten' vermischt, so dass der Zigarette menschliche Eigenschaften zugeschrieben werden.

Die Sätze wie (134) czuję przez bibułę, że coś się święci 'ich habe ein Vorgefühl, dass gleich etwas Schlimmes passiert', (137) Najpierw mnie podjarateś... 'zuerst hast du mich heiß gemacht' und der dazu gehörende weitere Teil (138) A teraz rzucasz 'und jetzt verlässt du mich' deuten auf die übertragene Bedeutung ihrer Elemente hin, d.h. podjarać als 'heiß machen' und nicht in der Grundbedeutung von 'anzünden' auftritt, so wie es bei im Falle von rzucać ist, wenn das Verb nicht in der Grundvariante erscheint 'wegwerfen' (aber in der Verbindung rzucać palenie 'mit dem Rauchen aufhören'), sondern als 'jdn verlassen'.

Die starken Gefühle werden mit der Aussage (139) że chciateś mnie tylko wykorzystać! 'Du wolltest mich nur ausnutzen!' oder (140) matka mi kazała cię rzucić! 'Meine Mutter ließ mich dich verlassen' betont.

Das Gespräch zwischen der Zigarette und dem Raucher erinnert an die Gespräche der Menschen, z.B. wenn folgende Aussagen formuliert werden (135) ja się wypalitem chyba! 'Ich habe jetzt keine Lust mehr, ich will nicht mehr', (136) Wypalitem...?, wo statt der Grundbedeutung von wypalić (papierosa) 'die Zigarette zu Ende rauchen' eben diese angeführte übertragene Bedeutung erscheint, aber mit diesem Unterschied, dass eben die Tatsache, dass eine Zigarette als eine Figur im Sketch erscheint, dieses Gespräch noch glaubwürdiger macht, wenn es um die Darstellung der Zigarettensucht geht. Der Zuschauer fühlt sich viel schneller angesprochen, als wenn man als Sketchfigur einen Arzt hätte, der über die Konsequenzen des Rauchens sprechen würde. Es ist somit den Autoren gelungen, alle Kontexte miteinander meisterhaft zu verbinden - es geht nicht nur darum, dass auch ein humoristischer Text eine belehrende Funktion haben kann, sondern auch darum, dass alles Problematische auch lustig präsentiert werden kann. Die Zielscheibe ist hier der Raucher, der nicht imstande ist, alleine mit seiner Sucht klar zu kommen und gegen sie etwas zu unternehmen. Das Motiv - das Gespräch zwischen dem Raucher und der Zigarette, das einem Gespräch zwischen den sich trennenden Partnern ähnelt, wird geschickt mit der präzise gewählten Lexik und natürlich mit allem Außersprachlichen, was auf der Bühne zu sehen ist, unterstützt. Der Kampf gegen die Sucht und die damit verbundenen menschlichen Schwächen bilden das Thema des Sketches. Die Pointe konnte doch hervorgesehen werden, denn so leicht hört man mit dem Rauchen nicht auf, der Mensch wird schwach und lässt sich von der Zigarette zu einem heimlichen Treffen überreden, wobei aber nicht nur eine Zigarette zum Treffen kommt, sondern sogar die ganze 
Schachtel, was im Satz (147) przyjdziemy cata paczka, ha! ausgedrückt wird, wo das Wort paczka nicht im Sinne von 'Clique' gebraucht wird, sondern als 'Zigarettenschachtel'.

Es ist ein Spiel nicht nur mit der Sprache, sondern auch mit der Situation, weil diese Art Abhängigkeit wie die innerhalb einer menschlichen Beziehung dargestellt und im Hintergrund auch kritisiert wird. Es werden auch stark emotional geprägte Wendungen gebraucht, die zusätzlich noch im Imperativ stehen oder als Anspielung einerseits auf das erwartete Verhalten des Rauchers - das Anzünden einer Zigarette - und andererseits auf seine Männlichkeit erscheinen, z.B. (143) rozpal mnie! 'Mach mich heiß!' und (145) To puść dymka, bądź mężczyzną!' Rauch eine Zigarette, sei ein Mann!' und (142) Masz mnie tu jak na tacy i nic, i nic?! 'Ich bin bereit und willig. Und du? Warum machst du nichts, warum nichts?'.

Da die Sketche als kurze Bühnenprogramme zu verstehen sind, fällt die $\mathrm{Zu}$ ordnung des Gesagten der Gruppe der Unterhaltungstexte viel leichter als wenn es nur um den geschriebenen Text ginge, denn in diesem Falle ist alles Außersprachliche mit im Spiel, steht sogar im Vordergrund, so dass der geschriebene und präsentierte Text um die Elemente der Körpersprache ergänzt wird und noch stärker wirken kann.

Jedoch wird die Witzigkeit [Kotthoff 1998b] in den dargestellten Texten auf solche Weise erzeugt, dass die Szenerie der lustigen Erzählung kreativ von dem Zuschauer bzw. Zuhörer rezipiert werden kann, was auf die entsprechende Wahl der Lexik und somit auf den sich daraus ergebenden Sinn zurückgeht. Der gelesene bzw. gesagte Text und das von dem Zuhörer bzw. Zuschauer assoziierte Bild werden schnell in dem Gesamtkontext platziert und somit richtig verstanden, d.h. das Komische wird erzeugt.

\subsection{Ethnowitze bei „Paranienormalni“" und „Ani Mru Mru'6}

Auch polnische Kabartettgruppen schreiben ethnische Witze, die im Vergleich zu den aus der Sendung „Was guckst du?“ jedoch auf bestimmte Nationen beschränkt sind. Meistens sind es Texte über Chinesen oder Vietnamesen, vielleicht deswegen, weil sich die Vertreter der genannten Nationen in Polen schon so eingelebt haben, dass sie auch zur Zielscheibe der Witze werden können. Das häufigste Motiv ist ein chinesisches Restaurant. Dieser situative Kontext wird für die humoristischen Zwecke u.a. von „Paranienormalni“ (,Wesele w chińskiej restauracji“) und von der Gruppe „Ani Mru Mru“ („Chińska restauracja“) ausgenutzt. 
Genau so wie im Falle der Sketchtexte mit Mariolka fällt es auf, dass die Texte im Vergleich zu den deutschen viel länger und was damit zusammenhängt - viel reicher an den sprachlichen Mitteln des Humoristischen sind.

Die Autoren berufen sich beim Schaffen ihrer humoristischen Texte vor allem auf die sich in den Gesprächen ergebenden sprachlichen Missverständnisse, die einerseits auf die fehlende Kenntnis der polnischen Sprache und ihrer Nuancen zurückzuführen sind und andererseits aus der fehlerhaften Aussprache resultieren. Somit entsteht auch ein Thema des Witzes - sprachliche Schwierigkeiten der Ausländer in Polen, die zu ihrem Alltag werden und sich leider nicht meiden lassen, vor allem wegen der totalen Verschiedenheit der Sprachsysteme - des Polnischen, des Chinesischen und des Vietnamesischen, vor allem auf der phonetischen Ebene.

Im Folgenden wird ein Sketchtext „Chińska restauracja“ von „Ani Mru Mru“ analysiert. Die beiden Figuren - der chinesische Kellner und der polnische Gast - haben vor allem Kommunikationsschwierigkeiten, die aus der dem Kellner fehlenden Kenntnis des Polnischen und auch aus der falschen Aussprache der polnischen Wörter resultiert. Sie verstehen einander nur wenig, trotzdem gelingt es den beiden, die Bestellung aufzugeben und anzunehmen.

\section{(79) Chińska restauracja}

Auf der Bühne sehen wir einen Gast sitzen und einen Kellner, der die Bestellung aufnehmen soll.Der Kellner trägt eine traditionelle chinesische Mütze, beugt sich vor dem Gast und grüßt ihn. K-Kellner, G-Gast

K: (1) Notieti nać pan! ... Hmmm... Notieti nać pan!! Aaaaaaa... Nasz klient nasz pan!! AAAAA... nasz klient nasz pan!! Nasz klient...

G: Dzień dobry.

K: (2)Nasz klient nasz pan!!!

G: Dobra, dobra, dobra, dobra. Co to jest?

K: (3a) tuteń.

G: Przepraszam, co?

K: (3b) tuuuuteńńńńńn.

G: Przepraszam, ale ja nie rozumiem. Pytam co to jest.

K: (4) Nie rosumie, nie rosumie. Se uczy to rosumie. Oo... (3c)

\section{tuteń? miska!}

G: Miska?! To jest bardzo dobry żart, bardzo śmieszne, bardzo!!

K:Hahahahahaha... Dziękuje...

G: Człowieku, ja nie pytam o to... tylko o to co jest w misce.

$K:$ (5a) Kutak w ciescie na otro.

G: Co takiego? 
K: (5b) Kuutakk w cieescie na otro!

G: (5c)Kurczak w cieście na ostro, tak?

K: Tak!

G: A tak się składa, że (6) z waszej ulotki, napisanej niby to po polsku, zamówiłem sobie UWAGA: (7a) kopytki ... w sosie.. na wynosi.. He!

K: (7b) My kopytki nie spsiedajemi.

G: Jak to... (7c) Co to znaczy my kopytki nie sprzedajemy? Co to w ogóle znaczy?? To dlaczego sa w waszej ulotce?!

K: (8) Chł... Chłu... Chłtł... Chłyt martetindodi.

$G: \dot{Z}$... że co, proszę??

K: (8a) Hihihihi... Chłłłaaaaaa... ... Chłyt martetindodi!!!!

G: (8b) Chwyt marketingowy?!

$K:$ Tak!!

G: (8c) Prosze pana, to jest dla mnie jakiś chiński chwyt marketingowy!

K: Ale działa!

G: Może i działa. Ja też zaraz zadziałam jak nie dostane czegoś normalnego do zjedzenia. (9) Ro-zu-miesz?

$\mathrm{K}:$ (10) Jak-sie-masz?

G: (11a) Hehehe... ping-pong?

K: (11b) Andrzej Grubba! Sto lat, sto lat!

G: Posłuchaj mnie Chińczyku. Posłuchaj mnie uważnie Chińczyku. Wszedłem tu bo byłem głodny i miałem blisko. Zamówiłem sobie jedno, dostałem zupełnie coś innego. Chce coś normalnego do jedzenia. Czy ty to rozumiesz?

K: (12)Nie wsystko. Pan młowi powolij. Ja się duszo domyślać.

G: Powoli?

K: Tak.

G: Dobrze... teraz będe mówił powoli. (13) A ty sobie weź jakiś mały chiński ołóweczek. I na malutkiej chińskiej karteczce sobie zapisz.

K: Tak.

G: Tylko dokładnie.

K: (14) Nasz klient nasz pan!

G: Tak, tak, tak, tak, tak... tak?

K: Tak!

G: Tak?

K: Tak.

G: ... schabowy.

K: Tak... (15a) niadodi. 
G: ... ziemniaki.

K: Tak... (15b) niotati.

G: I surówka...

K: Tak...

G: ... z czerwonej kapusty.

K: Oooo... tak! (16) Niututa so katuta... tak!

G: Tak.

K: Tak.

G: Tak.

K: Tak.

G: Tak.

K: Tak.

G: Tak.

K: Nie ma.

G: Powtórz to!

K: (17) Niiieee maaa...

G: Nie ma! To pytam po kiego grzyba bazgrolisz po tej kartce jak wiesz, że nie ma?! Skoro ci dyktuje schabowy, ziemniaki, surówka. To... (18) co to sq te czarne mróweczki, co te domki takie nastawiałeś. Co to jest w ogóle. Co to jest?

K: Sajgonki...

G: SAJGONKI?!

K: (19) Ryź z wadziwami.

G: Ja widzę ryż z warzywami jak patrzę na ciebie. Wygladasz jak wielki nieugotowany ryż z warzywami.

K: O! (20) Surówta z katusti!

G: Surówka z kapusty. Bardzo dobrze. Z jakiej? Czerwonej?

K: Pekińskiej...

G: W tym momencie moja cierpliwość się skończyła. Liczę do trzech i chce coś normalnego do jedzenia, jasne!

K: Tak.

G: Raz! Dwa! Trzy!

K: (21) Kocham cje...

G: (22) Ale ja nie potrzebuje twojej małej, skośnookiej, żółtej miłości. Tylko chce coś normalnego do jedzenia.

K: (23) Cielecina piec smaków.

G: Cielęcina pięć smaków. Dziękuję, już raz jadłem. Pół godziny szukałem mięsa między wiórkami bambusa... A kiedy już znalazłem okazało się, że to kalafior.

$K$ : To może (24a) łebtowina...

G: Co? 
$K:(24 b)$ Łebtowina...

G: Wieprzowina?

K: Tak!

G: Wieprzowina tak? A ile smaków 17 czy 61??

K: Niee...

G: Ja nie lubie chińskiej kuchni... wszedłem tu bo miałem blisko... (25) z chińskich rzeczy to lubie tylko podkoszulki... Zajrzyj do ulotki i znajdź mi coś normalnego do zjedzenia... to jest twoja ostatnia szansa...

K: Tak...

G: ... ostatnia szansa w życiu...

K: No to, no to może (26a)dzupa...??

G: Ale, że co (26b) dzupa?

K: (26c )Chińska dzupa... NIE!!! Nie o to chodzi... dzupa chińska...

G: Zupa?!

K: Tak... (26d) dzupa...

G: Zupa.

$K:(26 e)$ dzupa.

G: Zu-

K: (26f) Dzu-

$G$ und K: $(26 g)-p a .$.

G: Ale chińska nie! Nie jadłem chińskiej, nie jem i nigdy nie będę jej jeść...

$\mathrm{K}$ : Ale... ale...

G: Stop! Skończyłem dyskusję w tym momencie!! Nigdy nie...

K: Ale (26h) dzupa chińska, ale robiona w Radomiu! W ogóle nie smakuje jak chińska! G: W takim razie moge zaryzykować. Tylko raz-raz. No! Jak jest po chińsku raz-raz?

K: (27a) Ekekmewuzudu.

G: No (27b) to wuzudu, bo jestem głodny, jasne?

K: Tak.

G: A z czego ta zupa?

K: Nie wiem, nie napisano po chińsku na opakowaniu.

G: Pewnie z psa.

K: O nie, nie, nie. My z psa robimy cieleccine.

G: To aż boję się spytać co robicie z kota.

K: A z kota to kisiel i ciastka.

G: ?? A długo jeszcze będę czekał?

$K:$ (28) Tsy minuty!! Tsy minuty. Tsy...

G: To jakieś chińskie trzy minuty, chyba. 
K: (29) Już lece, pedze... dobra dzupa dobra... hehe... my presles... Aaaaaaaa... O kutwa!!

G: (30)I co narobiłeś kitajcu?! Co narobiłeś kitajcu?! Nie dość, że jestem głodny, zły to jeszcze oblewasz mnie ta cholerna zupa z Radomia! Ty, ty, ty, ty...

K: (31a) Hutank-tank-ze.

G: (31b) Hutanktankze?! A co to znaczy po chińsku?? (32a) Pan niescięscie?? Od dzisiaj będe tu przychodził codziennie, i ty codziennie będziesz mi robił suszi, aż całe spodnie wysuszi! Jasne!? Aha... i jeszcze jedno... (32b) zmień nazwe baru na niescięscie! (32c) Obsługa: pan niescięscie... (32d) Specjalność zakładu: niescięscie...

K: (32e) Tak... ja nietęcie! (33) Klient dzisiaj wielkie scięscie wielkie... (34) strach pomyśleć co by było, gdyby dzupa była goraca.

Der Chinese wiederholt die auswendig gelernten Sätze, die er entweder zur Begrüßung ausspricht (1) Nasz klient nasz pan!! 'Hier ist der Kunde König' oder als seine Rettung aus einer heiklen Situation versteht, wenn der Gast langsam schon die Geduld verliert (21) Kocham *cje...'Ich liebe dich'.

Interessant ist dabei die Tatsache, dass in diesem Text fehlerhafte Aussagen mit den korrekten vermischt werden, wie z.B. (33) Klient dzisiaj wielkie scięscie wielkie... oder (34) strach pomyśleć co by było, gdyby dzupa była goraca..., wo neben den falsch ausgesprochenen Elementen *scięscie statt szczęście 'Glück' und *dzupa statt zupa 'Suppe' , was auf Grund der Homophonie mit dem polnischen Wort dupa 'Hintern' assoziiert wird, auch grammatisch korrekte Formen erscheinen.$^{61}$ Das falsch ausgesprochene Wort *dzupa wird im Text mehrmals gebraucht, (26a-h) dzupa..., wobei von den Figuren auch mit der Sprache gespielt wird, indem das Wort in Silben zerlegt wird, d.h. (26f) Dzu- und (26g) - pa... und zum Schluss wird das polnische Produkt zupka chińska 'chinesische Instantsuppe` erwähnt, indem darüber gelacht wird, dass die Suppe keine echte chinesische Suppe ist, d.h. (26h) dzupa chińska, ale robiona w Radomiu! 'in Radom hergestellte chinesische Instantsuppe'.

In einer Textpassage wird von der Gastfigur ein falsch ausgesprochenes Wort (32a) niescięscie?? mehrmals in verschiedenen Verbindungen gebraucht, d.h. (32b) zmień nazwe baru na niescięscie! 'Benenne dein Restaurant um und nenne es niescięscie 'Unglück', (32c) Obsługa: pan niescięscie...'Bedienung ist ein

${ }^{61}$ Es erhebt sich an dieser Stelle die Frage, ob es Absicht oder vielleicht nur ein Versehen der Autoren war, was aber nichts an der Wahrnehmung des angebotenen Sketchtextes ändert. 
Unglück'(32d) Specjalność zaktadu: niescięscie... 'Spezialität des Hauses ist Unglück', was aber eine nicht erwartete Reaktion des Chinesen hervorruft, was wiederum davon zeugt, dass er die Aussage bis auf ein Wort nietęcie nicht verstanden hat, wovon die Bejahung (32e) Tak... ja nietęcie! zeugt.

Die Figur des Chinesen vermischt auch fremde Lexik mit der polnischen. z.B. (29) Już lece, pedze... dobra dzupa dobra... hehe... my presles... Aaaaaaaa... O kutwa!!, wo in einem Satz nicht nur fehlerhafte Formen des Polnischen *lece statt lece, *pedze statt pędze *dzupa statt zupa, *O kutwa!! statt O kurwa!, sondern auch die des Englischen *my presles statt my pleasure vorkommen.

Der Gast verwechselt die chinesische Küche mit der japanischen, indem er zum Kellner sagt (32a) Pan niescięscie?? Od dzisiaj będę tu przychodzit codziennie, i ty codziennie będziesz mi robit suszi, aż cale spodnie wysuszi!. In diesem Satz wird mit den Wörtern sushi und der falschen Form *wysuszi statt wysuszy gespielt, wobei es um die Bedeutung 'etwas trocknen' geht, was auf die phonetische Ähnlichkeit der genannten Elemente zurückzuführen ist.

Die ganze Geschichte ist voll von den fehlerhaften Wortformen, die eher phonetische Varianten sind, was aber doch lustig ist und den Rezipienten zum Lachen bringt, wie z.B. (1) *Notieti nać pan! ... Hmmm... Notieti nać pan!!, (3a-c)* Łuteń, (4) *Nie rosumie, nie rosumie. Se uczy to rosumie., (12) *Nie wsystko. Pan *młowi powolij. Ja się *duszo domyślać. [...], (7b) *My kopytki nie spsiedajemi und (8) ${ }^{*}$ Cht... Chtu... Chttl... Chtyt martetindodi. (8a) Hihihihi... *Chttlaaaaaa... ... Chlyt martetindodi!!!! statt chwyt marketingowy. (28) Tsy minuty!! Tsy minuty. Tsy... statt trzy minuty.

Der Chinese gebraucht auch fehlerhafte Formen der Bezeichnungen der in seinem Restaurant angebotenen Speisen, z.B. (7a-b) *kopytki w sosie... na wynosi... statt kopytka w sosie na wynos (5a-b) *Kutak w ciescie na otro. statt kurczak w cieście na ostro, '(19) *Ryź z wadziwami statt ryż z warzywami 'Reis mit Gemüse', (23) *Cielecina statt cielęcina, (20) *Surówta z katusti! statt surówka z kapusty und anstelle von wieprzowina erscheint (24a-b) *tebtowina.

Es gibt auch Elemente, die von dem Chinesen selbst erklärt werden (3a-b) Łuteń. als (3c) Euteń? miska! oder aber Wörter, die von dem Gast zuerst erfragt werden „,Jak jest po chińsku raz-raz?" als 'Wie heißt es auf Chinesich zackzack?’ und dann angeblich auf chinesisch wiedergegeben wird (27a) Ekekmewuzudu .

Es darf nicht außer Acht gelassen werden, dass der Chinese doch auch danach gefragt wird, ob er alles versteht (9) Ro-zu-miesz?, worauf er unerwartet mit der Frage (10) Jak-się-masz? antwortet und somit eine Kettenreaktion in Form von Frage und Antwort auslöst, d.h. auf die Frage (11a) Hehehe... ping-pong? reagiert der Chinese so, dass er sich auf sein Vorwissen aus dem Bereich Sport beruft und 
zusätzlich das typisch polnische zu jedem Anlass gesungene Lied „Sto lat!“ zu singen anfängt (11b) Andrzej Grubba! Sto lat, sto lat!

In diesem Sketch gibt es aber auch neben den falschen Wortformen völlig unverständliche Elemente, die erst von der anderen mitspielenden Figur - dem Gast - erklärt werden, so dass der Rezipient sie auch verstehen kann, z.B. (15a) *niadodi...als schabowy 'Schweinekotelett', (15b) *niotati als ziemniaki 'Kartoffeln', (16) *Niututa so katuta... als surówka z czerwonej kapusty 'Rotkohlsalat'. Nicht überraschend ist aber dabei die Reaktion des die Bestellung annehmenden Kellners, der gar nicht versteht, um welches Gericht es geht, d.h. kurze Antwort (17) Niiieee maaa...'Wir haben es nicht, wir führen es nicht'. Man könnte gleich annehmen, dass diese auf dem polnischen Gebiet erlernt worden ist, weil sie typisch polnisch ist. Demzufolge wird wieder auf die Stereotype gezeigt und mit ihnen gearbeitet. Die stereotype Denkweise stellt den Autoren ein breites Spektrum an Motiven und Themen zur Verfügung, was sie meisterhaft nutzen. Der situative Kontext ergänzt den sprachlichen und umgekehrt, so dass die Kommunikation zwischen den Darstellern und dem Publikum nicht gestört wird, obwohl es während des Gesprächs zwischen dem Gast und dem Chinesen zu den Missverständnissen kommt.

Das von den Rezipienten erwartete, sowohl mit der Sprache des Gastes und ihren Nuancen als auch mit den Gründen der im Sketch präsentierten komischen Situationen zusammenhängende Vorwissen impliziert das richtige Entschlüsseln der versteckten Inhalte aller Art - von der stereotypen Darstellung eines dienenden Chinesen, über seine sprachlichen Probleme bis zu den zahlreichen Anspielungen auf alles Chinesische, was aber nur bissig und ironisch gemeint wird. Die chinesische Nation wird mit den in China hergestellten Waren assoziiert (25) z chinskich rzeczy to lubię tylko podkoszulki... , ihrer leider nicht immer guten Qualität (8c) Prosze pana, to jest dla mnie jakiś chiński chwyt marketingowy!, 'für mich ist da leider ein chinesischer Werbeslogan' und mit der Größe der Chinesen, die in der Aussage auf alle chinesischen Gegenstände übertragen wird (13) A ty sobie weź jakiś mały chiński ołóweczek. I na malutkiej chińskiej karteczce sobie zapisz. ... 'Nimm einen kleinen chinesischen Bleistift! Auf einem kleinen chinesischen Zettel schreib...' Dabei wird auch die chinesische Schrift ausgelacht, die nicht zu entziffern ist (18) co to sa te czarne mróweczki, co te domki takie nastawiałeś? 'Was sind das für Zeichen, diese schwarzen Ameisen, diese Häuschen, die du gezeichnet hast?' und die schriftlichen Versuche des Chinesen, den Werbetext auf einem Flyer auf polnisch zu schreiben, werden in Frage gestellt (6) $z$ waszej ulot$k i$, napisanej niby to po polsku, was mit den von dem Gast zitierten Beispiel aus diesem Text belegt wird (7a) *kopytki ... w sosie.. na *wynosi... . Des Weiteren 
bewirkt das alles die Reaktion des Kellners und noch einmal wird eine fehlerhafte Äußerung (7b)* My kopytki nie spsiedajemi gebraucht, die dann doch von dem irritierten Gast wiederholt wird (7c) Co to znaczy my *kopytki nie sprzedajemy?

Es wird auch nicht auf den Gebrauch einer pejorativen polnischen Bezeichnung für einen Chinesen kitajec verzichtet (30) I co narobiteś kitajcu?! Co narobiteś kitajcu?! 'Und was hast du, Chinese, gemacht?'. Zum Schluss sagt der Gast dem Kellner direkt ins Gesicht, dass er auf seine angebliche Gastfreundschaft lieber verzichtet (22) Ale ja nie potrzebuję twojej matej, skośnookiej, żółtej miłości....'Ich brauche deine kleine chinesische schlitzäugige gelbe Liebe nicht!“" und so wie bei „Paranienormalni“" wird hier ein Quasivulgarismus gebraucht, nämlich kutwa.

In dem gerade anlaysierten und besprochenen Sketch wird deutlich gezeigt, wie stark die Unterschiede in der Darstellung der Relationen zwischen einem Polen und einem Ausländer sein können. Im Vergleich zu den Sketchtexten aus „Was guckt du?" fällt hier eher eine negative Einstellung zum Chinesen auf, was im ganzen Sketch mehrfach betont und wiederholt wird. Die Figur eines polnischen Gastes ist bissig, reagiert mit Ironie und Ärger auf die Versuche des Kellners, alles doch trotz der Sprachschwierigkeiten zu verstehen. Es kann hier nicht die Rede von der Sympathie und Verständnis sein, was natürlich nicht zu bedeuten hat, dass die Figur des Gastes als Vertreter der ganzen Nation erscheint und ihre Meinung vertritt.

Der auf der Bühne vorgetragene Sketch gewinnt dank der Körpersprache der daran teilnehmenden Darsteller an Witzigkeit. Der an sich schon lustige Text, der als eine relativ große Ansammlung von absichtlich von den Autoren entstellten sprachlichen Elementen besteht, wird geschickt mit der nonverbalen Sprache so ergänzt, dass der Zuschauer keine andere als erwartete Reaktion zeigen kann, indem er in Gelächter ausbricht, weil das Komische als eine gelungene Mischung des Sprachlichen und des Situativen zu verstehen ist.

Im Rahmen des bereits analysierten Textes werden vor allem fehlerhafte Formen gebraucht, die den phonetischen Formen der jeweiligen Wörter entsprechen, es gibt demzufolge ein andauerndes Spiel mit der polnischen Sprache, deren Erlernen so viele Schwierigkeiten in sich birgt, dass es sogar zum Thema eines witzigen Textes werden kann.

Dabei darf nicht vergessen werden, dass es von Kultur zu Kultur verschieden ist - das Tabu ist national, politisch, religiös und sozial bedingt. So kann man sich u.a. in Polen nicht vorstellen, dass man Witze über Behinderte oder über ältere Leute, über die Krankheiten und den Tod schreibt. In jeder Kultur sind die sog. dirty jokes, schmutzige Witze oder nieprzyzwoite, świńskie kawały/żarty zu fin- 
den. Als Witze dieser Art dienen sie auch dazu, tabuisierte Themen zu entschärfen und somit die in der jeweiligen Kultur geltenden Tabus zu verletzen und zu durchbrechen. Es darf nicht außer Acht gelassen werden, dass in jeder Kultur andere Themen ,verboten“ sind, was uns aber doch feststellen lässt, dass vor allem auf die Sexualität als Tabuthema hingewiesen wird:

In mediterranen Kulturen dominieren sexuelle Witze, während in den nord-westlichen Lädern Europas vorrangig skatologische Witze zu finden sind. Auch Schimpfwöter entstammen in süd-östlichen Kulturen dem Bereich des Sexuellen, während sie im Nordwesten vorrangig dem Wortfeld des AnalFäkalen entnommen werden [Dimova 2008, S. 17]. ${ }^{62}$

Aus der Analyse der deutschen Witze geht hervor, dass es dort kaum Tabus gibt - es wird über alles und über jeden gelacht. Der Sinn für Humor ist nämlich auch national bedingt, es wird doch zwischen dem britischen, deutschen und polnischen Sinn für Humor unterschieden, die im Wesentlichen andere Merkmale haben, was auf das allgemeine Charakteristikum des jeweiligen Volkes zurückgeht, was natürlich auf den Stereotypen basiert, d.h. britischer Humor ist eher absurd, deutscher Humor ist zu direkt und wird sogar als anal bezeichnet, weil Deutsche eine besondere Vorliebe für die geschmacklosen Witze zeigen, Polen dagegen werden mit solcher Humorart assoziiert, die entweder politisch, gegen Kirche gerichtet ist oder eher erotisch als geschmacklos zu bezeichnen wäre.

${ }^{62}$ ska|to|lo|gisch <Adj.>: 1. (Med., Paläont.) die Skatologie (1) betreffend. 2. (Psych.) eine auf den Analbereich bezogene Ausdrucksweise bevorzugend. 2000 Dudenverlag. 


\section{INTERTEXTUALITÄT IN DEN DEUTSCHEN UND POLNISCHEN SKETCHTEXTEN}

Bei der Analyse der humoristischen Texte, die dank den Medien bekannt werden, muss noch auf eine weitere Erscheinung hingewiesen werden, die auch mit den Medien zusammenhängt. Es geht um die Intertextualität, die in diesem Falle alle Fernsehformate anbetrifft - von einer Nachrichtensendung bis zur Werbung. Die Witze, die geschrieben werden, entstehen auch unter dem Einfluss des Gesehenen und des Gehörten, d.h. können sich demzufolge auf bestimmte Fernsehprogramme bzw. Radiosendungen beziehen und als ihre Parodie innerhalb der humoristischen Texte erscheinen. Nicht weniger oft berufen sich die Autoren der Texte auf die den Rezipienten bekannten Filme, deren Titel oder sogar die Helden aufs Neue den Zuschauern oder Zuhörern präsentiert werden, indem sie sie zum Lachen bringen.

Diese Erscheinung ist sowohl im Rahmen der deutschen als auch der polnischen humoristischen Texte zu sehen. Man kann sogar wagen zu sagen, dass es eine internationale Erscheinung ist, was wiederum sich doch einfach erklären ließe. Der heutige Rezipient hat Zugang zu allen möglichen Medien, wird täglich mit Millionen Informationen konfrontiert und zum Schluss noch soll er unterhalten werden. Diese letzte Aufgabe wird durch das Fernsehen, das Radio oder das Internet erfüllt, so dass die dort zu findenden Formate eben zu den Unterhaltungszwecken eingesetzt werden. Damit ein neu entstehender komischer Text sein Ziel erreichen kann, sollte er sich nicht nur auf die abstrakten Sachen beziehen, sondern sich eher darauf berufen, was dem Rezipienten schon irgendwoher bekannt vorkommt. Da der Mensch von heute rund um die Uhr fernsehen, im Internet surfen bzw. verschiedene Radiosendungen hören kann, scheint es sicher einfacher zu sein, das Bekannte zu parodieren als etwas Neues, was dem Rezipienten noch nicht bekannt ist, zu schreiben. Im polnischen Film „Rejs“, dem Kultfilm der 70er Jahre, wurde sogar schon darauf hingedeutet, dass der Mensch nur dann ins Kino geht und sich nur diese Filme anschaut, die er kennt. So scheint demzufolge auch die Unterhaltungsindustrie zu funktionieren - man beruft sich eben in erster Linie auf alles Bekannte, vor allem wegen der einfachen und vorauszusehenden erwarteten Interpretation. Im Falle der Intertextualität geht es um den Bezug jedes ein- 
zelnen Textes auf die schon vorhandenen anderen Texte. ${ }^{63}$ Es ist demzufolge die Relation zwischen den Texten - den schon bekannten und den erst erschienenen. Mit diesem Begriff wird erklärt, dass die entstehenden Texte verschiedenartige Relationen zu anderen schon vorhandenen aufweisen, zu denen u.a. solche gehören, die aus der traditionellen Rhetorik bekannt sind, nämlich

- Anspielung,

- Kommentar,

- Ironie und

- Parodie.

Als Referenztexte, also solche, auf die Bezug genommen wird, funktionieren

- Werbetexte,

- Titel und Formate der Fernsehshows oder aber auch

- Titel und Formate der Filme.

Die für die Zwecke dieser Arbeit analysierten humoristischen deutschen und polnischen Texte weisen auch diese Eigenschaft auf. Im Rahmen des deutschen Comedyprogramms „Was guckst du?“ wird mit folgenden Fernsehformaten gespielt:

- „Tagesschau“664 wird als „Tagesguck“ präsentiert.

- „Wer wird Millionär?“65 dient als Ausgangspunkt für den deutschen Integrationstest.

- Nach dem Vorbild der Show „Jeopardy“66 erscheinen in „Ausländer-Jeopardy“ drei Spieler (alle drei gespielt von Kaya Yanar), die ihre Antworten in Form einer Frage formulieren müssen

- In der Flirtshow „Herzblatt“67 sucht ein machohafter Italiener Francesco nach der Liebe seines Lebens

- „Richterin Ünal“ ist die Entsprechung der deutschen Fernsehprogramms „Richterin Barbara Salesch“68.

${ }^{63}$ Dieser Begriff wurde von Kristeva eingeführt. Vgl. Schweikle G., Schweikle I. [1990, S. 223].

\footnotetext{
${ }^{64}$ ARD-Nachrichtensendung

${ }^{65}$ RTL-Quizshow

${ }^{66}$ RTL-Quizshow

${ }^{67}$ Das Erste - und ORF-Flirtshow

${ }^{68}$ SAT.1 - Gerichtsshow
} 
Die Autoren beziehen sich auch auf die Werbung, die im deutschen Fernsehen läuft:

- Das Format der Werbung für die Haarkosmetikreihe „Drei-Wetter-Taft“ wird für die Werbung für einen BMW genutzt, indem im Programm die Werbung für „Dreier-Fahrt“ erscheint

oder aber schaffen neue Werbungen, die dem Format nach den schon laufenden ähnlich sind, z.B. Werbung für den deutschen Duft (,Deutsch-Duft“), in der es um das Parfüm geht, das dem Ausländer die Freundschaft der Deutschen garantiert, weil es solche Komponenten wie Bockwurst-, Schweiß- und Zigarettenduft enthält. Diese Mischung soll aus einem Ausländer einen Deutschen machen. Die einleitenden Worte erinnern an die Worte fast jeder Werbung, d.h. es erscheinen am Anfang einige Fragen, die als rhetorische Fragen zu verstehen sind -,,Fühlen Sie sich allein? Haben Sie keine Freunde? “, was noch mit der Körpersprache des Ausländers betont wird, weil er langes Gesicht macht, einen traurigen Blick hat, seine Augenbrauen hochzieht, was auch als Zeichen der Verwunderung zu verstehen ist, die als Reaktion auf eine so gestellte Fragen erscheint. Des Weiteren sind noch andere Elemente der Körpersprache im Spiel, wenn das neue Produkt „Deutsch-Duft“ präsentiert wird - der Ausländer ist jetzt glücklich, er strahlt vor Freude, was man an dem Gesichtsausdruck und seinen lächelnden Augen erkennen kann.

In den in „Was guckst du?“ gezeigten Werbungen erscheinen meist Ausländer, es gibt aber eine Werbung für „Turk-Deutsch-Simulator“, in der als Hauptfigur ein Deutscher zu sehen ist. Wieder fängt die Werbung mit der Frage an, die aber diesmal an einen Deutschen gerichtet wird:

\section{(80) Werbung für "Turk-Deutsch-Simulator"}

Kennen Sie das? Niemand versteht Sie, keiner weiß, was Sie wollen... und das alles, weil Sie die Sprache Ihres Heimatlandes nicht beherrschen.

Mit dem Turk-Deutsch-Simulator versteht man Sie auch in Deutschland wieder. Richtig angewendet, verwandelt der TurkDeutsch-Simulator Ihr verstaubtes Hochdeutsch in lupenreines Asi-Turk-Deutsch.

Somit wird auch auf die Situation in Deutschland angespielt, d.h. darauf, dass die fremden Einflüsse in der deutschen Sprache so stark sind, dass sogar die Kommunikation im Alltag erschwert ist. Es ist die Werbung für ,einen Turk-Deutsch- 
Simulator", der als Hilfe beim Gespräch zwischen einem Ausländer als Türsteher und einem Deutschen, der in die Disco will, dienen soll.

Die entstellte, bisher korrekte deutsche Sprache wird schnell mit diesem einfachen Gerät zum Türkisch-Deutsch und die Alltagsgespräche können ab sofort normal verlaufen.

Als ein anderes Beispiel dieser Art kann die Werbung für „den Navigator“ verstanden werden, die mit folgendem Text anfängt: „Sie kennen das Problem? Ihr veraltetes Navigationssystem drückt sich nicht genau aus? Vergessen Sie es! Ihr neues Turk-Deutsch-Navigator zeigt Ihnen, wo es lang geht! ". Auf diese Weise wird dem Fahrer ein neues Gerät angeboten, mit dem der richtige Weg auf Türkisch-Deutsch beschrieben wird und sogar an alles Wichtige wird der Fahrer aufmerksam gemacht, z.B. wenn er beim Aussteigen vergisst das Licht auszumachen „Hey, Alde, macht das Licht aus! Ey, bist du taub?? Mach das Scheiße-Licht aus!“

In einer weiteren Folge wird die Werbung für das Navigationssystem fortgesetzt, in der der am Steuer sitzende Nazi ein besonderes Gerät im Auto hat, nämlich „,den Nazigator“". In diesem Falle wird mit den Wörtern gespielt, weil das Wort Navigator als Basis für neue Lexeme dient - Turk-Deutsch-Navigator und Nazigator. Im ersteren Falle liegt eher eine Bezeichnung vor, die per analogiam zu Turk-Deutsch-Simulator gebildet wurde und im letzteren - wird der Buchstabe [v] zu [z] und somit kann sich auch der Fahrer angesprochen fühlen, der ein Nazi ist und demzufolge einen Nazigator haben muss. Das Motiv der Nazis ist nicht selten in „Was guckst du?“ zu sehen. Das Verhalten dieser Gruppe wird parodiert und ausgelacht. In dieser Werbung spielt auch das Außersprachliche eine wichtige Rolle, vor allem aber fällt das Erscheinungsbild der Figur auf - der Fahrer trägt eine schwarze Lederjacke, ist kahlköpfig, sieht wie „ein typischer Nazi“ aus und genau so reagiert er auf die Befehle des Navigationssystems mit „Jawohl!“ und hat dann zum Schluss einen Unfall, was wiederum als eine Anspielung auf die Nazis zu verstehen wäre, die blind die Befehle des Führers ausführten.

Im Falle der polnischen humoristischen Texte der Gruppe „Paranienormalni“ sollen vor allem folgende Merkmale genannt werden, die auf den intertextuellen Charakter ihrer Witze hinweisen

- Parodien der Filme „M jak miłośćc“69 und „Rocky“70

- Anspielungen auf die Werbung für Baumaterialien

- Gebrauch der Markenzeichen, wie z.B. McDonald's

\footnotetext{
${ }^{69}$ Polnischer Serienfilm.

${ }^{70}$ Amerikanischer Actionfilm.
} 
Auch im Falle von „Ani Mru Mru“ können noch weitere Elemente genannt werden, die mit der Intertextualität zusammenhängen, d.h.

- Gebrauch des geläufigen Slogans der polnischen Anti-Gewalt-Kampagne „Bo zupa była za stona“ als Ausgangspunkt für den Satz, der im Sketch der Gruppe „Ani Mru Mru“ erscheint (,,Strach pomyśleć co by było, gdyby zupa byta goraca!")

- Gebrauch der Formel vom Typ „Klient nasz pan!“ ('Hier ist der Kunde König') im Sketch von „Ani Mru Mru“, die von einem Chinesen gebraucht wird und als Rettung aus einer heiklen Situation verstanden wird.

An dieser Stelle sollte demzufolge noch einmal betont werden, dass eben dieser intertextuelle Charakter der humoristischen Texte von den Rezipienten ein relativ umfangreiches Vorwissen aus verschiedenen Bereichen verlangt, so dass im Endeffekt der Sinn der Sketchtexte richtig entschlüsselt, verstanden und dann auch interpretiert werden kann. Das Fehlen der genannten Kenntnisse impliziert demzufolge entweder falsches Verstehen der Texte oder sogar ihr Nichtverstehen. Bei der Wahrnehmung und beim Entschlüsseln der in den humoristischen Texten versteckten Inhalte ist der Rezipient auf sein eigenes Wissen angewiesen, das von den Autoren angenommen und gleichzeitig verlangt wird.

Sowohl in den Sketchtexten aus „Was guckst du?" als auch in den Witzen von der Gruppe „Paranienormalni“ wird unter anderem auf Folgendes angespielt:

- Werbungen, z.B. in „Was guckst du?“ - Dreier-Fahrt in Anlehnung an die Werbung für die Kosmetikreihe Drei Wetter Taft,

- bestimmte Filme, z.B. in „Was guckst du?“ - Szenenserie „Nicht mit meiner Tochter“, wo der Titel des Filmes „Nicht ohne meine Tochter“ paraphrasiert wird, und bei „Paranienormalni“ „M jak młotek“ statt „M jak miłość“

- Fernsehsendungen, wie z.B bei Kaya Yanar „Wer wird Millionär?“ (es wird in dem Sketch „Deutscher Integrationstest“" nachgeahmt) oder ,Jeopardy“, wo Yanar selbst in drei Figuren schlüpft und als ein Russe, ein Italiener und ein Türke an dem Quiz teilnimmt.

Die Kenntnis der einzelnen für die Zwecke der Comedy ausgenutzten Fernsehinhalte wird von den Autoren vorausgesetzt, kann aber auch den Rezipienten fehlen, was sich demzufolge im eventuellen Missverständnis oder sogar dem Nicht-Verstehen der jeweiligen Texte manifestiert. Anders ist es im Falle der alltäglichen Komik, wo man sich auf das vorhandene Gruppenwissen beruft, das das Verstehen des Komischen ermöglicht. 
Es ist dabei noch die Rede von den Scherzaktivitäten, wobei auf verschiedene Staretegien der Komik gezeigt wird [Kotthoff 2004a, S. 6]. Kotthoff nennt 30 Scherzaktivitäten, die für nicht-ernste Kommunikation und somit auch für witzige Texte charakteristisch sind, wobei betont werden soll, dass man es in den meisten Fällen mit den hybriden Formen zu tun hat, weil selten in einem Witz nur eine Scherzaktivität festzustellen ist.

1. Wortspiele/Verfremdungen und Konventionen

2. Witzige Bemerkungen

3. Absurde Theorien

4. Anekdoten

5. Necken

6. Frotzeln

7. Pflaumen/Aufziehen/Schmäh

8. Ironie und Selbstironie

9. Absurde Phantasien

10. Konversationelle Grotesken

11. Konversationelle Karrikaturen

12. Konversationelle Parodien

13. Narrative Witze

14. Rätselwitze

15. Sarkastische Aktivitäten

16. Scherze auf eigene Kosten

17. Sexuelles Witzeln

18. Sich-Mokieren

19. Spaßige Aufwertungen von Nichtigem und Banalem

20. Spaßige Anspielungen

21. Spaßiger Rollentausch

22. Spaßige Schilderungen

23. Spott

24. Verulken/Veräppeln

25. Verwischungen von Medien- und Realwelt

26. Schwarzer Humor

27. Blödeln

28. Running Gag

29. Lächerlich machen/Verarschen

30. Nonverbale Komik, zum Beispiel Grimassen 
Die Witze, die nach der Zielscheibe (Blondinen, Polizisten, Beamten, Lehrer, verschiedene Nationen), dem Thema (Dummheit, Leben im Ausland, Verhalten am Arbeitsplatz) oder dem Motiv, das oft gleichzeitig auch die Zielscheibe und das Thema sein kann, klassifiziert werden können, arbeiten mit allen genanten Scherzaktivitäten, die miteinander ein außergewöhnliches Format ergeben, so dass man auch im Rahmen der Comedy-Programme zu ihnen greift, um in Verbindung mit allem Außersprachlichen das Komische zu schaffen. In der Sendung „Was guckst du?" und auch in den Programmen von „Paranienormalni“ und „Ani Mru Mru“ ist zu sehen, dass die genannten Formate meist vermischt sind, so dass sich aus dieser Mischung im Endeffekt ein abwechslungsreiches Programm ergibt. Die für die Zuschauer bestimmten humoristischen Szenen verbinden das Verbale mit dem Nonverbalen, wobei dem letzterem eine besondere Rolle zugeschrieben steht, weil eben diese Scherzaktivität alles Gesagte unterstreicht und verstärkt.

Das Sexuelle verkörpert in „Was guckst du?" Francesco, ein sexbessener Italiener, der sich auf Kosten der Frauen amüsiert. In den Sketchen von „Paranienormalni“" wird dieses Motiv dagegen u.a. in den Szenen mit Mariolka mitberücksichtigt, z.B. Mariolka1, wenn die Figur von einem LKW-Fahrer erzählt, der sie fragte, ob sie ihm seine Ladung ausladen helfe, was doppeldeutig ist - es kann wortwörtlich verstanden werden oder aber auch im sexuellen Sinne von 'Geschlechtsverkehr'.

Die Gruppe „Ani Mru Mru“ hat in ihrem Programm auch einen Sketch mit Gienek, der sein ,erstes Mal“ haben soll. Der ältere Bruder führt ihn in die Situation ein. Der Satz Ojciec to zrobit, ja to zrobitem, teraz kolej na ciebie. 'Der Vater tat das, ich tat das, jetzt bist du dran. ' lässt die Rezipienten glauben, dass es um Sex geht. Das Gespräch wird von den beiden so geführt, dass der Zuschauer hundertprozentig daran glaubt, dass es um das erste sexuelle Erlebnis geht. Zum Schluss hören wir noch ein bekanntes Motiv von Jean Michelle Jarre, das als Motiv des Sieges gilt. Gienek erscheint glücklich auf der Bühne und dann kommt das Unerwartete - es war das erste Mal, dass er sich von einem Fotoautomaten fotografieren ließ. Das hat der Rezipient gar nicht erwartet und somit ist es den Autoren gelungen, eine richtige Pointe zu schaffen und das Publikum mit dem unerwarteten Ende so zu überraschen, dass es lacht.

In den erwähnten Sketchen werden verschiedene Scherzaktivitäten ausgenutzt, sie beinhalten Elemente aller genannten Typen, von den absurden Theorien und witzigen Bemerkungen, über Necken, Aufziehen, sexuelles Witzeln, Veräppeln, Anspielungen bis zu den Wortspielen. Das alles wird noch mit der nonverbalen Komik unterstützt, so dass sich zum Schluss um eine besondere, einzigartige Art Humor handeln kann. 



\section{KÖRPERSPRACHE ALS ELEMENT DES HUMORISTISCHEN}

In der Literatur zur nonverbalen Kommunikation wird in erster Linie ihre Funktion im Kommunikationsprozess und ihre Bedeutung für die an diesem Prozess beteiligten Gesprächspartner betont. Heutzutage geht man davon aus, dass die Kenntnis der Körpersprache, das richtige Dekodieren der in den nonverbalen Signalen versteckten Inhalte und das bewusste Einsetzen aller Elemente der Sprache des Körpers die Voraussetzung für den beruflichen und gesellschaftlichen Aufstieg sind [Matschnig 2007a, 2011a, 2011b, 2012]. Es werden sogar zahlreiche Seminare angeboten, die den angehenden Führungskräften die Macht dieser Signale zeigen und ihnen beibringen, wie man sich dieses Wissens bewusst bedient, so dass man als glaubwürdig, vertrauenswürdig und erfolgreich eingeschätzt wird.

Es wird dabei nicht außer Acht gelassen, dass das Sprachliche mit dem NichtSprachlichen eine Einheit bildet, so dass auf diese Weise eine Botschaft vollständig weitergeleitet werden kann.

Die Körpersprache, ihre Bedeutung, Funktionen und Einsatzmöglichkeiten sollen demzufolge als ein wichtiger Bestandteil des Kommunikationsprozesses verstanden werden, in dem es zur Mitwirkung des Sprachlichen und des Außersprachlichen kommt, so dass die zu sendenden Informationen möglichst genau und treu vermittelt werden.

Im Vergleich zu den geschriebenen Texten, deren Verständnis einerseits aus dem Entschlüsseln der zwischen den Zeilen versteckten lustigen Informationen und andererseits aus dem für jeden Rezipienten charakteristischen Vorwissen resultiert, steht noch ein weiterer Faktor im engen Zusammenhang mit der Wahrnehmung eines gesprochenen Textes, nämlich die Körpersprache [Ekman 2004; Heidemann 2009; Mühlisch 1997, 2007; Henley 1989, 2007].

Interessant scheint aber die Tatsache zu sein, dass die Psychologen auch zwischen verschiedenen Arten der Körpersprache innerhalb einer und derselben sozialen Gruppe sprechen, nämlich - über die Körpersprache der Frauen und die der Männer. Nach den zahlreichen Forschungen stellte man fest, dass es sog. frauen- und männerspezifische Verhaltensmuster gibt und demzufolge auch - ver- 
schiedene Systeme von nonverbalen Zeichen. Es geht dabei um Unterschiede in den Körperbewegungen, im Augenkontakt und in den Berührungen und der damit verbundenen räumlichen Distanz. ${ }^{71}$

Für die Interpretation der auf der Bühne aufzutragenden Sketchtexte sind alle nonverbalen Signale genauso wichtig wie die verbalen. Nicht nur die Art und Weise, auf die das Lustige präsentiert wird, also alle mit der Aussprache verbundenen Erscheinungen, wie die Stimmführung, die Stärke der Stimme, die Satzmelodie, der Akzent und die Aussprache der einzelnen Laute, Silben und Worte, sondern auch das Erscheinungsbild der Darsteller spielen dabei eine sehr wichtige Rolle. Die Interpretation solcher humoristischen Texte erfolgt demzufolge auf zwei verschiedenen Ebenen - der bewussten und der unbewussten. Damit meine ich in erster Linie die Tatsache, dass sich der Mensch als Kommunikationspartner auf der sprachlichen Ebene sicherer bewegt als auf der Ebene der nonverbalen Signale, weil er meint, die Sprache und ihre Nuancen zu verstehen, wobei er mit dem Verstehen der körperlichen Zeichen doch Probleme haben kann, was vor allem auf ihren individuellen Charakter zurückgeht. So wie für jeden von uns ein konkretes Idiolekt charakteristisch ist, so ist für uns auch ein bestimmtes eigen- und einzigartiges nonverbales Verhalten typisch. Wir senden verschiedene Signale, die auch unterschiedlich verstanden und interpretiert werden, was nicht selten zu den Missverständnissen führen kann. Die Gesprächspartner sind dazu gezwungen, sich nicht nur auf das Sprachliche zu konzentrieren, sondern auch Schlüsse aus dem nonverbalen Verhalten ihrer Gegenüber zu ziehen. Das Nonverbale wird zum größten Teil unbewusst eingesetzt, weil es auf die Emotionen zurückgeht und sich nicht ganz steuern lässt. Die Körpersprache wird der Sprache der Gefühle gleichgesetzt, woraus das intuitive Verstehen der Intentionen unserer Kommunikationspartner resultiert. Es darf aber nicht außer Acht gelassen werden, dass alle nicht-sprachlichen Signale manchmal doch auch mit Absicht in ein Gespräch eingeführt werden, damit auf diese Weise ein erhofftes Ziel erreicht wird.

Bei der Präsentation auf der Bühne ist vor allem das wichtig, wie der jeweilige Text vorgetragen wird, weil eben diese unbewussten Signale des menschlichen Körpers inhaltsreicher sind als die Worte selbst. Unser Verhalten, die Körperhaltung, die Augen, der Mund - das alles, was als Ausstrahlung definiert wird, beeinflusst es, wie man von seinen Kommunikationspartnern wahrgenommen wird. Auch das Erscheinungsbild - die Kleidung, die Frisur und sogar das benutzte Parfüm erzeugen ein gewisses Bild von uns, das von dem Gesprächspartner gespei-

${ }^{71}$ Es wird zwischen der intimen ( 0 bis 40 Zentimeter), vertrauten bzw. persönlichen (40 Zentimeter bis 1,5 Meter), gesellschaftlichen (1,5 Meter bis 4 Meter) und der öffentlichen Distanz (ab 4 Meter) unterschieden. 
chert wird und demzufolge auch später in dieser und nicht in einer anderen Form abberufen wird, unabhängig davon, ob es tatsächlich der Wahrheit entspricht oder aber irreführend ist. Dabei spielt noch ein weiterer Faktor eine Rolle - der so genannte erste Eindruck. Innerhalb von Sekunden wird entschieden, wie wir von einem Fremden gesehen, wahrgenommen und auch in seinem Gedächtnis gespeichert werden, ob mit unserem Bild positive oder negative Emotionen (Sympathie, Liebe, Zuneigung vs. Sarkasmus, Ironie, Aggression, Hass) assoziiert werden. Mit den Gesten, dem Gesichts- und Augenausdruck, die nicht immer kontrolliert werden, vermitteln wir viel mehr Informationen als mit dem Text selbst, weil dabei unsere Gefühle mit im Spiel sind. Die genannten Elemente der nonverbalen Kommunikation werden als Träger der Botschaft verstanden.

Wie es schon früher erwähnt wurde, wird das Nonverbale zum größten Teil unbewusst produziert, demzufolge bleibt jedem von uns als Gesprächspartner noch ein Teil übrig, der das umfasst, was erlernt werden kann. Durch das Beobachten anderer Menschen und ihres Verhaltens kann man gewisse Reaktionen nachmachen und sie dann bewusst und mit Absicht in einer bestimmten kommunikativen Situation als seine eigenen ausnutzen, um bei seinen Gesprächspartnern gut anzukommen. Nicht selten kommt es nämlich vor, dass wir auch unbewusst nicht nur die Körpersprache einer anderen Person, sondern auch ihre Sprache nachahmen und uns auf diese Weise an die konkrete kommunikative Situation anpassen.

Die Wirkung der Signale ist besonders in den Kontakten mit den Fremden von Bedeutung, unabhängig davon, ob damit andere Kulturkreise oder aber auch eine andere soziale Gruppe gemeint wird. Alle nonverbalen Signale hängen einerseits mit der Intention des Gesprächspartners zusammen und andererseits sind sie Reaktionen auf die Äußerung der anderen Person. Das Verstehen dieser Signale hängt jedoch auch davon ab, in welchem Kulturkreis wir uns als Sprecher befinden. Die Geste und die Mimik können demzufolge manchmal auch verschieden interpretiert werden. Dabei wird dem Körperkontakt eine wichtige Rolle zugeschrieben, denn es wird zwischen den so genannten kontaktarmen und kontaktreichen Kulturen unterschieden, worunter als erstere die Südeuropäer und als letztere die Asiaten verstanden werden. Für die genannten Gruppen ist u.a. eine andere Kontaktdistanz typisch, d.h. in den kontaktreichen Kulturen ist diese geringer, was in ihren Kontakten mit einer kontaktarmen Kultur zu den Missverständnissen und zu verschiedenen Problemen führen kann. Schwierigkeiten solcher Art können auch im Rahmen der Kommunikation auftauchen, was eben aus der falschen Interpretation der nonverbalen Signale in Verbindung mit dem Verständnis des Gehörten resultiert. Dabei soll noch darauf hingewiesen werden, dass die Dekodierung der nonverbalen Signale nicht immer gelungen ist, was darauf zurückgeht, dass auch die Körpersprache etwas Individuelles und somit auch 
für jeden einzelnen Menschen typisch ist. Als Beispiel kann man an dieser Stelle das bekannte Augenzwinkern nennen, das verschieden interpretiert werden kann, je nachdem, wann, von wem und natürlich zu welchem Zweck es eingesetzt wird. Wenn unser Gesprächspartner seine Aussage mit dem Augenzwinkern abschließt, können wir daraus schließen, dass er es entweder nicht ernst gemeint hat oder das Gesagte mehrdeutig sein kann. Aber wenn der Zuhörer auf die gehörte Information mit dem Augenzwinkern reagiert, kann es bedeuten, dass er die Ehrlichkeit der Sprechers bezweifelt und somit das Gehörte in Frage stellt. Interessant ist dabei folgende Bemerkung,

Wenn man längst vergessen hat, was Sie gesagt haben, wird man sich immer noch daran erinnern, welches Bild Sie abgegeben haben. ${ }^{72}$

mit der eindeutig darauf gezeigt wird, dass die nonverbale Kommunikation genauso wichtig wie die verbale ist, aber manchmal doch wichtiger als sie zu sein scheint.

Im Rahmen der Forschungen über die nonverbale Kommunikation wird eben das betont, dass man zwischen der unbewussten, teilbewussten und der bewussten nonverbalen Kommunikation unterscheidet.

Da schon die unbewusste und die bewusste nonverbale Kommunikationsart angesprochen wurden, muss noch auf die teilbewusste eingegangen werden. In dem letzteren Fall geht es darum, dass der Gesprächspartner sich dessen bewusst ist, wie und wann, unter dem Einfluss welcher Situationen und Menschen sich seine Mimik im Verlauf eines Gesprächs ändert, ob er traurig, aggressiv, verlegen oder zufrieden und glücklich ist und auf alles Lustige, was nicht nur mit der Sprache zum Ausdruck gebracht wird, z.B. mit dem Lächeln oder sogar mit dem lauten Lachen reagiert.

Dem Lächeln wird noch eine andere Funktion zugeschrieben - die des Mittels der Kontaktaufnahme. Nicht ohne Bedeutung ist auch, in diesem Zusammenhang auf das Lachen hinzuweisen, das als eine besondere Ausdrucksform innerhalb der nonverbalen Kommunikation zu verstehen ist. Mit dem Lächeln und dem Lachen reagiert man nicht nur auf etwas Komisches, es kann auch sein, dass es die Reaktionen der Verlegenheit sind und in diesem Falle dient das Lachen der Entspannung und der Entkrampfung einer für uns unangenehmen Situation. Das wird meisterhaft im Rahmen der scherzhaften Kommunikation ausgenutzt, wenn ein humoris-

${ }^{72}$ Wie man ehrliches Lächeln erkennt, von: Körpersprache. Ein Lächeln wirkt Wunder [2007], (=http://www.focus.de/finanzen/karriere/management/koerpersprache/koerpersprache/koerpersprache_aid_5470.html). 
tischer Text, der für die Bühne geschrieben wurde, von dem Darsteller so mit allen möglichen nonverbalen Elementen ergänzt und unterstützt wird, dass im Endeffekt das Publikum zu lachen anfängt. Das Ziel der scherzhaften Kommunikation wird somit erreicht. Da werden konkrete Rollen gespielt, die mit bestimmen Witzfiguren zusammenhängen. Die Schauspieler wirken auf die Zuschauer überzeugend, weil sie sich mit der Rolle identifizieren und sich in sie hineinversetzen. Auf der Bühne versuchen sie möglichst treu die Merkmale der jeweiligen zu spielenden Figur darzustellen, indem von ihnen die für sie charakteristische Sprache gebraucht wird, die dabei noch mit den nonverbalen Signalen unterstützt wird. Die außersprachlichen Elemente hängen einerseits mit der Rolle zusammen, andererseits aber sind sie mit dem Darsteller selbst verbunden, so dass daraus eine Mischung aus den nonverbalen Elementen entsteht, die zusammen mit dem präsentierten Text das Komische erzeugt, die Zuschauer erheitert und sie zum Lachen bringt.

Neben dem Text, der voll von mehrdeutigen Elementen ist, deren Bedeutungsvarianten vermischt und mit Absicht verwechselt werden, bekommt der Zuschauer von „Was guckst du?" auch ein bestimmtes Bild zu sehen - die vorzutragenden Rollen werden von den Darstellern meisterhaft mit ihrer Mimik, Körperbewegung, Stimme und mit ihrem Erscheinungsbild unterstützt. Konkrete Figuren, gespielt von Yanar, verlangen ein bestimmtes Image - von einer russischen Hellseherin Olga, die mit ihrer Kugel die Zukunft vorhersehen kann, über einen türkischen Fahrlehrer Yildirim, der ständig mit seiner Schirmmütze erscheint, seinen deutschen Schüler veräppelt, indem er seinen Namen nur selten gebraucht, weil er dabei mit den Assoziationen spielt (Manfred Winter wird Schneemann, Weihnachtsmann, sogar Rutschpartie genannt), Hakan in der Bomberjacke und mit einer aufgestylten Frisur, der sich in den Schritt greift, wenn er nervös wird und dann den Satz Hey du, du kommst hier nicht rein! gebraucht, der immer lächelnde und die Zuschauer mit einem Hallo begrüßende Randjid mit seiner Kuh Benita, der die deutsche Realität erst näher kennen lernt, der italienische Macho Francesco, mit viel Gel im Haar, schick angezogen, immer auf der Jagd nach Frauen, Tarek ab del Kalek, der arabische Journalist, der verschiedene Events besucht und Interviews durchführt und dabei meist auf arabisch spricht und die von ihm gedolmetschten Phrasen entsprechen nur selten der Wahrheit, denn der Zuschauer kann in den Untertiteln die deutsche Version lesen, so dass man genau sehen kann, dass die interviewten Personen einfach veräppelt werden (u.a. Dieter Bohlen, der Habibi 'Schatz' wiederholt) und Süleyman, der im Radio arbeitet, desssen Studio wie ein Rummelplatz aussieht, neben dem im Korb sein Hund Süleyhund sitzt und der den Zuhörern die Ratschläge gibt. In diesem Falle liegen demzufolge fast alle genannten Scherzaktivitäten vor, die in einem Sketch miteinander vermischt werden, so dass man auf einmal mit verschiedenen Komik- 
verfahren arbeitet. Nicht weniger wichtig ist die Hintergrundmusik, als die dann einerseits in den meisten Fällen bekannte Hits gewählt werden - von Presley in „Was guckst du?“, von Danzel bei „Paranienormalni“ oder von Jarre bei „Ani Mru Mru“ und andererseits auch Motive aus anderen Fernsehsendungen, wie z.B. „Wer wird Millionär?“, womit der intertextuelle Charakter der humoristischen Texte dieser Sendung betont wird.

Die Gienek-Figur („Ani Mru Mru“) erinnert dem Erscheinungsbild nach an einen etwas intellektuell zurückbleibenden Menschen, der komisch angezogen ist - zu kurze Hose, hoch gezogen, eine komische Schirmmütze auf dem Kopf, aufgesetzte Brille mit der altmodischen Fassung, der Mensch, der etwas seltsam spricht, sich schämt, etwas zu sagen und dazu noch Zähne wie ein Hase hat. Diese Mischung macht das Außersprachliche aus, das von den Autoren meisterhaft als Hintergrund des Sprachlichen eingesetzt wird, so dass sich die beiden Komponenten ergänzen und das Humoristische zum Ausdruck bringen.

Die Mariolka-Figur („Paranienormalni“), in die Igor Kwiatkowski schlüpft und sie meisterhaft darstellt, verdankt zwar ihre Wirkung dem vorzutragenden Text mit dem außergewöhnlichen Stil und der Lexik, die eine Mischung aus Elementen der polnischen Umgangssprache, der Jugendsprache und den von mir so genannten Quasivulgarismen ( $k u w a)$ ist, aber sie wird in erster Linie mit ihrem Aussehen assoziiert - nicht nur mit ihrem blonden Haar (womit auch die Blondinenwitze mit im Spiel sind), sondern auch mit dem gestreiften Pullover in grellen Farben und einer umgehängten kleinen Tasche. Auf der Bühne bewegt sie sich hektisch, hüpft, läuft hin und her und das alles wird mit Musik von Danzel begleitet, so dass auch das Intertextuelle miteinbezogen wird. Im Rahmen der Szenen mit Mariolka haben wir es mit dem narrativen Witz zu tun, weil die Figur uns von ihren Erlebnissen der vergangenen Tage erzählt und dabei ein Telefongespräch mit ihrer Freundin Gabryśka führt. In dem genannten Sketch wimmelt es von

- absurden Theorien (mcDonald's-Test),

- falsch oder gar nicht verstandenen fremdsprachigen Wörtern (banjo verwechselt mit bungee, bungalow verstanden als eine Art Unterwäsche),

- nicht bekannten polnischen Wörtern, die in einer Wortgruppe homophon sind (strefa bezctowa verstanden als strefa bez stowa),

- doppeldeutigen Lexemen pory 'Hautporen' vs. pory 'übergroße Hose', die dann in Verbindung mit dem Verb ściagać 'zusammenziehen' vs. ściagać 'herunterziehen, ausziehen' in der Wendung ściagać pory erscheinen und demzufolge auch verschieden interpretiert werden sollten, 
was doch dann eindeutig gemacht wird, indem Mariolka die außersprachlichen Elemente gebraucht und mit der Körperbewegung zeigt, dass es eben um die Hose geht, obwohl es die Rede von einer Creme ist. Auch das Banale wird scherzhaft aufgewertet, indem einfache Alltagstätigkeiten zu etwas Außergewöhnlichem werden. Der Einsatz der Körpersprache unterstreicht das im Text zum Ausdruck gebrachte Komische und das Ziel dieser Art Kommunikation wird erreicht - es wird gelacht.

Die kreative Wahrnehmung der Realität, die für die Autoren von „Paranienormalni” charakteristisch ist, gibt dem Rezipienten die Möglichkeit, weitere Kontexte im Rahmen der dargebotenen humoristischen Szenen zu bilden. Wir als Zuschauer wirken an allem mit, weil der Doppelsinn der ausgedachten Sketchtexte erst dann entschlüsselt und richtig interpretiert werden kann, wenn man bewusst daran teilnimmt, um die versteckten Informationen und die lustigen Inhalte zu finden, sie richtig einzustufen und als Elemente des Humoristischen zu verstehen. Interessant scheint dabei die Tatsache zu sein, dass auch in diesem Falle das Modell des konversationellen Humors eingesetzt wurde, das sich aus dem Sprachhumor und dem Sachhumor zusammensetzt.

Bei der Dekodierung der in den Witzen jeder Art versteckten Inhalte und Informationen und bei ihrer Interpretation spielt das Allgemeinwissen des Rezipienten eine enorm große Rolle. Es wird das Grundwissen aus verschiedenen Bereichen als Voraussetzung für das Verstehen der geschaffenen humoristischen Texte verlangt. Die Autoren bedienen sich dabei eines breiten Spektrums von Motiven, Figuren und Themen. Die sprachlichen und außersprachlichen Mittel verhelfen ihnen dabei zur Bildung und dann zur Präsentation einer fiktiven Realität, unabhängig davon, wer oder was ausgelacht wird. Die Gruppe „Paranienormalni" kommentiert keine politischen Ereignisse, sie konzentriert sich vor allem auf die menschlichen Schwächen und Verhaltensweisen, zeigt die Alltagssituationen, indem sie typisch polnisches Verhalten im Zerrspiegel darstellt. Geschickt wird dabei die polnische Sprache, ihre Nuancen, die manchmal echt komplizierten Regeln, die Mehrdeutigkeit und die stilistische Färbung als Mittel des Humoristischen eingesetzt. Nicht ohne Bedeutung ist gleichzeitig der außersprachliche Kontext, der von Igor Kwiatkowski geschaffen wird. Durchdachte, sprachlich interessant konstruierte Texte, unterstützt mit allen Details der Körpersprache, mit der Sprechweise und natürlich auch mit dem Kostüm und der Maske machen das Komische aus. Der sprachliche Kontext, in Verbindung mit dem außersprachlichen, gibt den Autoren die Möglichkeit, ihr Ziel zu erreichen - den Zuschauer zum Lachen zu bringen. 



\section{SCHLUSSFOLGERUNGEN UND PROGNOSEN}

Sowohl für die Sendungen dieser Art wie „Was guckst du?“, als auch für die Sketche von den polnischen Gruppen „Paranienormalni“ und „Ani Mru Mru“ ist vor allem das Wortspiel als das wichtigste sprachliche Mittel des Humoristischen zu nennen. In seinem Rahmen wird vor allem mit der Polysemie gespielt, womit nicht nur die semantische Mehrdeutigkeit, sondern auch die auf der phonetischen Ebene gemeint werden. Es wird mit Hilfe der semantischen und phonetischen Ambiguität auf Kosten anderer gelacht, was mit der übertriebenen Darstellung der ausgedachten witzigen Figuren verbunden ist. Diese Vertreter von verschiedenen Nationen, Kulturen, sozialen Gruppen und Berufsgruppen werden manchmal mit Biss, aber gleichzeitig mit viel Sympathie und Zuneigung präsentiert, so dass, obwohl es etwas riskant klingt, der Zuschauer auf diese Weise neue Freunde gewinnt, die ihn regelmäßig beim Fernsehen begleiten. Was sucht man in diesen Gestalten? Der Rezipient versucht vor allem in den kennen gelernten Figuren etwas von seiner Persönlichkeit zu finden, besonders dann, wenn diese mit viel Humor präsentiert werden, der meist zwischen den Zeilen gefunden und entschlüsselt werden soll, manchmal aber auch direkt ist.

Die durchgeführte Analyse der ausgewählten deutschen und polnischen Sketchtexten hat Folgendes ergeben:

- In „Was guckst du?“ wird eine besondere Art Witz angeboten - der Ethnowitz, der die ganze Sendung determiniert und sogar zum Markenzeichen dieses Programms geworden ist. Dieses Programm gilt als erste Ethno-Comedy in der deutschen Medienlandschaft. Der Charakter der angebotenen Witze impliziert den Einsatz von fehlerhaften Wortformen, Wendungen und sogar den ganzen Sätzen, was auf die fehlenden Deutschkenntnisse der darzustellenden Witzfiguren zurückgeht. Die Sprache der Figuren als ein hybride Form aus fremdsprachigen Varianten des Deutschen wird zum weiteren Erkennungszeichen der Sendung.

- Die Darstellung des Multikulturellen mit Hilfe des Humoristischen hat zum Ziel, die Einwanderergesellschaft dem deutschen Publikum näher zu bringen, ohne nur eine von vielen in jeder der ausgedachten Witzfiguren stilisierten 
Nationen besonders scharf zu kritisieren und auszulachen. Das macht das Besondere der Sendung aus.

- Der Rezipient der Texte von „Paranienormalni“ bekommt dagegen eine Mischung aus Witzen verschiedener Art zu sehen und zu hören, wobei darauf hingewiesen werden kann, dass diese Gruppe vor allem mit der Figur von Mariolka assoziiert wird, die die jungen Menschen von heute besonders anspricht, obwohl sie eigentlich bei jedem Zuschauer, unabhängig vom Alter, beliebt ist, wovon die Reaktionen des Publikums zeugen.

- Für deutsche und polnische Texte ist es charakteristisch, dass sie ihre eigenen Formate haben, mit denen auch u.a. auf die Fernsehsendungen, Filme, Werbungen, bestimmte Musikmotive, bekannte Namen und Marken angespielt wird. Somit kann sowohl im Falle der polnischen („M jak miłość“ „,Rocky“, Anspielungen auf die Werbung für Baumaterialien, Gebrauch der Markenzeichen, wie z.B. McDonald's) als auch der deutschen Sketchtexte („Tagesschau“, „Wer wird Millionär?“, „Jeopardy“, „Herzblatt“, „Richterin Barbara Salesch“, Werbung für die Haarkosmetikreihe „Drei-Wetter-Taft“) von der Intertextualität die Rede sein.

- Sowohl im Deutschen als auch im Polnischen steht das Wortspiel als Mittel des Humoristischen an erster Stelle. Der situative Kontext, bezogen auf die deutsche bzw. die polnische Realität, betont mit allem Nonverbalen, wird von den Autoren mit den für die Zwecke des Humoristischen eingesetzten sprachlichen Mitteln unterstützt, so dass sich daraus eine in sich zusammenhängende witzige Einheit ergibt.

- Interessant ist dabei, dass in den beiden Sprachen vor allem mit der Polysemie, der Homonymie und der Homophonie gespielt wird. Die angebotenen witzigen Texte setzen eher die Kenntnisse der umgangssprachlichen Varianten der beiden Sprachen voraus und gehen nicht auf die Literatursprache des jeweiligen Sprachsystems zurück, in der es auch zahlreiche stilistische Mittel gibt, mit denen das Witzige zum Ausdruck gebracht werden könnte.

- In den beiden Sprachen wird genauso gern und oft zur Polysemie als Mittel des Humoristischen gegriffen, wobei darauf hingewiesen werden soll, dass sehr oft damit gespielt wird, dass die mehrdeutigen Lexeme neben ihrer fachlichen Grundbedeutung auch eine übertragene Bedeutungsvariante haben, die eben in den witzigen Texten erscheint.

- Die Autoren von „Paranienormalni“ spielen geschickt mit der Fachlexik, die neben ihrer fachlichen Bedeutung auch eine übertragene Bedeutungsvariante hat, wobei sich die Witzfiguren eher der letzteren bedienen, was aber erst aus dem Gesamtkontext zu erschließen ist und demzufolge von den Rezipienten sowohl die Sprach- als auch Fachkenntnisse verlangt (u.a. zwinać się als 'weggehen, jdn 
verlassen', kleić się ‘gut verlaufen, gut funktionieren', znosić coś 'etw. ertragen, aushalten`, drzeć się `sehr laut schreien` oder aber odejść od kogoś `jdn verlassen, sich von jdm trennen' bzw. skuć się `sich besaufen', rozklejać się 'weich werden, gerührt sein, in Tränen ausbrechen', wypalić się 'keine Lust mehr haben, etwas nicht mehr wollen', pustak 'dumme Frau' usw.)

- Im Vergleich zu den deutschen Sketchtexten, fallen die von den polnischen Autoren durch die fachlichen Ausdrücke auf, deren Bedeutung dem Rezipienten bekannt sein soll, was das notwendige Vorwissen impliziert (u.a. francuz 'Franzose, verstellbarer Schraubenschlüssel ', wiartarka z udarem/udarowa 'Schlagbohrmaschine', ściana nośna 'Tragwand')

- An dritter Stelle steht die Homophonie, wobei darauf hingewiesen werden soll, dass es doch schwer einzuschätzen ist, welche der beiden Sprachen sich häufiger der homophonen Formen bedient, aber anhand der analysierten Sketchtexte konnte festgestellt werden, dass der Gebrauch solcher phonetisch ähnlichen Formen als Element des Witzigen vom Thema der angebotenen Texte abhängig ist. Im Polnischen fallen die homophonen Formen vor allem in den Mariolka-Sketchen, was auf das fehlende Sprachkenntnis der Figur zurückgeht, sei es das des Polnischen (u.a. strefa bez stowa statt strefa bezclowa, sczepić się statt szczepić się, laleczka lubudu statt laleczka woodoo, magnez 'Magnesium' statt magnes 'Magnet', oder aber auch das des Englischen bungee statt banjo).

Im Deutschen dagegen geht es um solche Ähnlichkeiten, die sich z.B. aus der Zusammenstellung der den Sketchfiguren nicht bekannten deutschen Einzellexeme und der ganzen Wortgruppen ergeben (u.a.na, Sie wissen... verstanden als Nazis).

- Die Autoren der Texte von „Paranienormani“ bevorzugen die Umgangssprache als Mittel zum Ausdruck der witzigen Inhalte (alle Mariolka-Szenen mit solchen Elementen wie u.a. übertrieben oft gebrauchter Quasivulgarismus kuwa, Vulgarismus spieprzałam 'ich bin so schnell weggelaufen', zahlreiche Elemente der polnischen Jugendsprache und der Umgangssprache zugleich Hejka! 'Hallo!', * ja pitole 'nicht schlecht, Scheiße!', zarąbiście /zarąbisty/ zarąbiste, rewelacja als 'super, toll!', jaka siara 'was für Schande!', pojechana na maxiora ty krejzolko als 'Du bist so verrückt!', bez kitu 'echt!', Kuwa, ja nie moge!! 'Mann, das trage ich nicht mehr aus!', spoko pieniadz 'ganz schön viel Geld!', brechtać się 'lachen', balety 'Party', nie mieć prawka 'keinen Führerschein haben').

Dabei werden auch Wörter fremder Herkunft eingesetzt, die von der Figur oft entweder nicht korrekt ausgesprochen oder sogar falsch verstanden werden (u.a. helot 'Hör auf, erzähl keinen Unsinn!', *a propo statt a propos, no sex, Wiadomo o co kaman! 'Man weiß, worum es geht'). Als Fremdwörter im engeren Sinne 
Happy Birthday und no sex werden in ihrer Originalform gelassen. Es erscheinen auch fremdsprachige Eigennamen der von McDonalds angebotenen Produkte (cheeseburger, Happy Meal).

Vor allem in den Mariolka-Szenen fallen die für diese Figur typischen Floskeln auf, die als Unterbrechungselemente gelten (Quasivulgarismus kuwa, co nie? 'nicht wahr?', helot und Czujesz to?! 'Verstehst du das?').

- Sowohl im Deutschen als auch im Polnischen werden in den Sketchtexten die Vulgarismen gebraucht, wobei sich die deutschen Autoren auf das Wort Schei$\beta e$ ! beschränken, die polnischen dagegen zu den sog. Quasivulgarismen greifen, die wegen ihrer homophonen Form kuwa und kutwa den polnischen Vulgarismus kurwa ersetzen und auf diese Weise das Lustige im Text auch noch stärker betonen.

- Auch die Wortbildung des Deutschen kann als sprachliches Instrument des Witzigen verstanden werden. Mit Hilfe der Komposition, vor allem aber nach dem Modell des Determinativkompositums, werden in der deutschen Sprache solche Lexeme gebildet, die als Neologismen aufzufassen sind und in den jeweiligen witzigen Szenen als Verstärkungsmittel aller lustigen Inhalte auftauchen, was vor allem auf ihren besonderen emotionalen Wert zurückgeht. Im Falle der Sendung „Was guckst du?“ hat das noch einen anderen Grund - es soll nämlich noch stärker betonen, wie schwer es für einen Ausländer sein kann, alle Regeln der für ihn bisher unbekannten Sprache zu verstehen, sich diese anzueignen und zum Schluss noch - korrekt anzuwenden. Das beste Beispiel dafür haben wir im Sketch (64. Türkischer Dolmetscher beim Bewerbungsgespräch), wo zum Ausdruck der Empörung eben neue Wörter gebildet werden, d.h. Scheißjob, Dreckkohle und Scheiß-dreck-arsch-scheißjob. Das ist eine nur für die deutsche Sprache charakteristische Erscheinung.

- Die Annahme, das das Polnische als Mittel des Humoristischen eine stärker emotional gefärbte Sprache zu verstehen ist, hat sich demzufolge bestätigt, wovon zahlreiche polyseme Lexeme mit den emotional gekennzeichneten, für die Zwecke des Humoristischen eingesetzten Bedeutungsvarianten zeugen, die in den polnischen Sketchtexten auftauchen.

- Die Analyse der ausgewählten Texte hat deutlich gezeigt, dass der Humor doch einerseits etwas Neutrales sein kann, das unabhängig von der Sprache und von der Zeit existiert und als eine universelle Erscheinung wahrgenommen werden kann, wobei andererseits seine Ausdrucksmittel und die damit zusammenhängenen Möglichkeiten der Interpretation doch etwas Subjektives darstellen, was vom Rezipienten zum Rezipienten verschieden aussehen kann und dazu noch auf das jeweilige Sprachsystem und seinen emotionalen Wert zurückgeht, woraus eben die emotionale Ladung der witzigen Texte resultieren kann, die nicht nur 
mit dem Außersprachlichen, sondern auch mit der bestimmten Lexik ausgedrückt werden kann.

Da sich sowohl die polnische als auch die deutsche Kabarettszene in den letzten Jahren ziemlich schnell entwickeln und weil immer wieder neue Tetxe mit aktuellen Inhalten entstehen, kann die konfrontative Analyse der beiden Sprachsysteme fortgesetzt werden, damit gezeigt werden kann, ob die Vereinfachung der Lexik, unabhängig vom Sprachsystem als die sog. sprachliche Globalisierung verstanden werden kann, was in erster Linie auf die immer an Bedeutung zunehmenden Entlehnungen, vor allem aus dem Englischen, zurückzuführen ist.

Man kann des Weiteren auch der Frage nachgehen, ob die Sprache als Quelle der Mittel zum Ausdruck des Humoristischen an Bedeutung gewinnt oder verliert und welche Rolle demzufolge dem Nonverbalen im Rahmen der scherzhaften Kommunikation im Laufe der Zeit zugeschrieben wird. Um auf die so gestellten Fragen zu antworten, müsste man dann davon ausgehen, dass man nach den Sendungen bzw. Bühnenprogrammen sucht, die, wie in dem in diesem Buch besprochenen Falle, ähnliche, vergleichbare Formate und Inhalte anbieten würden. Interessant wäre auch das Problem der Rolle der neu übernommenen fremdsprachigen Elemente aus verschiedenen Bereichen als Mittel des Humoristischen, was davon zeugen würde, dass sich diese in dem jeweiligen Sprachsystem entweder gut oder vielleicht gar nicht eingebürgert haben.

Die Analyse der Sprache als Mittel des Humoristischen in den beiden Sprachsystemen könnte auch das Problem der witzigen Inhalte in der polnischen und der deutschen Werbung betreffen, die uns doch auf Schritt und Tritt begleitet.

Man kann demzufolge zu dem Schluss kommen, dass eben der Bereich des Humoristischen ein ziemlich breites Spektrum der weiteren Forschungsthemen darstellt, die aufgeriffen werden können. In diesem Bereich wären vor allem die weiteren Analysen auf der konfrontativen Ebene Deutsch vs. Polnisch besonders interessant. 



\section{BIBLIOGRAPHIE UND QUELLENVERZEICHNIS}

Apte M.L. [1985], Humor and Laugher. An Anthropological Aproach, Ithaca/London Arnu T. [1997], Da lacht der Autor, in: Süddeutsche Zeitung, Kulturmarkt, Nr. 84, S. 7-8. Attardo S. [1994], Linguistic Theories of Humour, Berlin/New York

Awdiejew A. [2005], Strategie konwersacyjne, in: Acta Universitatis Lodziensis. Folia Litteraria Polonica 7, S. 127-150.

Awdiejew A. [1991], Technologia dowcipu a proces twórczy, in: Potrzeby, twórczość, przyszłość, Wrocław, S. 227-239.

Awdiejew A. [1991], Strategie konwersacyjne (próba typologii), in: Socjolingwistyka XI, Katowice, S. 7-20 .

Awdiejew A. [1992], Nieśmieszne aforyzmy (Refleksja nad semantyka humoru Viktora Raskina), in: Nowakowska-Kempna, I. (Hrsg.), Podstawy metodologiczne semantyki współczesnej, Język a Kultura 8, Wrocław, S. 279-285.

Bachmeier H. (Hrsg.) [2007], Lachen macht stark. Humorstrategien, Göttingen.

Bartmiński J. (Hrsg.) [2001], Współczesny język polski, Lublin.

Bartmiński J. [2001], Styl potoczny, in: Bartmiński J. (Hrgs.), Współczesny język polski, Lublin, S. 115-134.

Bartmiński J., Szadura J. (Hrsg.) [2003], Współczesna polszczyzna. Warianty języka, Lublin.

Bausinger H. [2000], Typisch Deutsch. Wie deutsch sind die Deutschen?, München.

Bergson H. [1972], Das Lachen. Ein Essay über die Bedeutung des Komischen, Zürich.

Best O.F. [1989], Der Witz als Erkenntniskraft und Formprinzip, Darmstadt.

Best O.F. [1993], Ein Volk ohne Witz. Über ein deutsches Defizit, Frankfurt a.M.

Bondzio W. (Hrsg.) [1980], Einführung in die Grundfragen der Sprachwissenschaft, Leipzig.

Borew J. [1960], Über das Komische, Bonn.

Böhler M. [1981], Die verborgene Tendenz des Witzes. Zur Soziodynamik des Komischen, in: Deutsche Vierteljahresschrift 55, S. 351-387.

Bremmer J., Rodenburg H., (Hrsg.) [1999], Kulturgeschichte des Humors - Von der Antike bis heute, Darmstadt.

Brock A. [2006], Was wandelt sich am Komischen? Comedy-Formate unter Veränderungszwang. Abstract des Referats, Kasseler Komik - Kolloquium 21. bis 26. Februar 2006, Kassel.

Brzozowska D. [2000], Uczucia w dowcipach, in: Nowakowska-Kempna I. (Hrsg.),Uczucia w języku i tekście, Język a Kultura 14, Wrocław, S. 285-294.

Brzozowska D. [2004], Wpływ dowcipów amerykańskich na dowcipy polskie, Stil 3, Beograd, S. 429-435. 
Brzozowska D. [2005], Agresja w dowcipach, in: Dąbrowska A., Nowakowska A. (Hrsg.), Agresja i życzliwość w języku i kulturze, Język a Kultura 17, Wrocław, S. 231-236.

Bußmann H. [1983], Lexikon der Sprachwissenschaft, Stuttgart.

Buttler D. [1961], O dowcipie słowotwórczym, in: Poradnik Językowy, z. 3, S.7.

Buttler D. [1971], Odmiany polskiej homonimii, in: Poradnik Językowy, z. 1-2.

Buttler D. [1980], Typy polisemii przymiotników polskich, in: Slavia 49.

Buttler D. (Hrsg.) [1984], Stownik polskich form homonimicznych, Wrocław.

Buttler D. [2001], Polski dowcip językowy, Warszawa.

Bystroń J. [1960], Komizm, Wrocław.

Cherubim D. [2008], „Selten so gelacht ... “ Darf man über Sprachfehler lachen?, in: Hoffmann T., Lercher M-Ch., Middeke A., Tittel K. (Hrsg.), Humor. Grenzüberschreitende Spielarten eines kulturellen Phänomens, Göttingen, S. 181-194.

Cialdini R. [2000], Wywieranie wptywu na ludzi. Teoria i praktyka, Gdańsk.

Cui P. [2008], Deutscher und Chinesischer Humor - eine kontrastive Studie zu deutschen und chinesischen ethnischen und Familienwitzen, Bremen.

Czeszewski M. [2006], Stownik polszczyzny potocznej, Warszawa.

Detering K. [1983], Zur linguistischen Typologie des Wortspiels, Tübingen

Dimova A. [2008], Humor und Witz als Übersetzungsproblem, in: Hoffmann T., Lercher M-Ch., Middeke A., Tittel K. (Hrsg.), Humor, Göttingen, S. 7-20.

Drews G. [2005], Das extra dicke Witzbuch, Augsburg

Driessen H. [1999], Lachen und Feldforschung: Betrachtungen aus dem Blickwinkel der Ethnologie, in: Bremmer J., Roodenburg H. (Hrsg.), Kulturgeschichte des Humors, Darmstadt, S. 167-185.

Drösser Ch. [1998], Linguisten wissen, warum wir über Witze kichern: Der Scherz lebt vom Widerspruch, von:http:// www.zeit.de/ (Stand vom 14.06.2010).

DUDEN [2000], Das große Wörterbuch der deutschen Sprache, CD-Rom, Mannheim.

Duszak A. [1998], Tekst, dyskurs, komunikacja międzykulturowa, Warszawa.

Dżereń-Głowacka S., Kwiatkowska A. (Hrsg.) [2009], Humor. Teorie - praktyka-zastosowania. Zrozumieć humor, Piotrków Trybunalski.

Dżereń-Głowacka S., Kwiatkowska A. (Hrsg.) [2008], Humor. Teorie-praktyka-zastosowania. Odcienie humoru, Piotrków Trybunalski.

Ekman P. [2004], Gefühle lesen - Wie Sie Emotionen erkennen und richtig interpretieren, München

Fąka P. [2008], Parametr „abstrakcyjność-rubaszność” w humorze językowym. in: Dżereń-Głowacka S., Kwiatkowska A. (Hrsg.), Odcienie humoru. Humor - Teoria. Praktyka. Zastosowania, Piotrków Trybunalski, S. 83-91.

Fiedler S. [2003], Sprachspiele im Comic. Das Profil der deutschen Comic-Zeitschrift Mosaik, Leipzig

Filipiak M. [2003], Homo communicans. Wprowadzenie do teorii masowego komunikowania, Lublin.

Fischer-Fabian S. [1992], Lachen ohne Grenzen. Der Humor der Europäer, Frankfurt a.M.-Wien.

Frahm T. [2008], Verneigung vor dem Säbel. Gibt es einen bulgarischen Humor?, in: Hoffmann T., Lercher M.Ch., Middeke A., Tittel K. (Hrsg.), Humor, Göttingen, S. 24

Freud S. [1993], Dowcip i jego stosunek do nieświadomości, Warszawa. 
Freud S. [1985], Der Witz und seine Beziehung zum Unbewußten, Frankfurt a.M.

Freud S. [1982], Der Humor. Studienausgabe, Bd. IV, Frankfurt a. M.

Gabler Wirtschaftslexikon, Stichwort: Massenkommunikation, http://wirtschaftslexikon. gabler.de/Archiv/55023/massenkommunikation-v5.html

Gajda S., Brzozowska D. (Hrsg.) [2000], Świat humoru, Opole.

Garski R. [2008], ,,Der mit dem Wort tanzt “. Kreativer Gebrauch von Phraseologismen in Zeitungsüberschriften, in: Der Sprachdienst 3, 52, S. 105-116.

Grassegger H. [1985], Sprachspiel und Übersetzung. Eine Studie anhand der Comicserie Asterix, Tübingen

Grochala B. [2006], Komizm językowy w felietonach Antoniego Stonimskiego, Łódź.

Grochala B. [2004], Dowcip językowy na stronach www., in: Michalewski K. (Hrsg.), Współczesne odmiany języka narodowego, Łódź, S.111-120.

Grochala B. [2005], Dowcip językowy w nagłówkach ,, Gazety Wyborczej”, in: Acta Universitatis Lodziensis. Folia Linguistica 43, S. 25-35.

Grochala B. [2007], Językowe przejawy stereotypów w dowcipach, in: W dialogu języków i kultur, Warszawa, S. 417-425.

Grochala B. [2008], Kraj się śmieje, czyli z czego śmieja się dziś Polacy? Analiza komizmu językowego współczesnych tekstów kabaretowych, in: Cygal-Krupa Z. (Hrsg.), Wspótczesna polszczyzna - stan, perspektywy, zagrożenia, Kraków-Tarnów, S. 343351.

Grochala B. [2008], Od Zielonego Balonika do Ani Mru Mru - kabaretowa tradycja i nowoczesność (aspekty językowe), in: Woźniak E. (Hrsg.), Tradycja a nowoczesność, Łódź, S. 469-478.

Grochala B. [2009], Z kogo śmieja się Polacy-tematyka dowcipów na przestrzeni dziejów, in: Bieńkowska D., Lenartowicz A. (Hrsg.), Tajemnice rozwoju, Łódź, S. 147-156.

Grochala B. [2009], Inne oblicze dowcipu politycznego, in: Dżereń-Głowacka S.,

Kwiatkowska A. (Hrsg.) [2009], Humor. Teorie - praktyka-zastosowania. Zrozumieć humor, Piotrków Trybunalski, S. 49-57.

Grochala B. [2010], Intertekstualność jako element budujacy teksty o zabarwieniu

Humorystycznym, in: Mazur J., Matyska A., Sobstel K. (Hrsg.), Intertekstualność we współczesnej komunikacji językowej, Lublin, S. 135-142.

Glück H. (Hrsg.) [1993], Lexikon Sprache, Stuttgart.

Grassegger H. [1985], Sprachspiel und Übersetzung. Eine Studie anhand der ComicsSerie Asterix, Tübingen.

Gruntz-Stoll J. [2001], Ernsthaft humorvoll. Lachen(d) Lernen in Erziehung und Unterricht, Beratung und Therapie, Bad Heilbrunn.

Grzegorczykowa R. [2001], Wprowadzenie do semantyki językoznawczej, Warszawa.

Hallstatt M. [2002], Faule Witze von A bis Z, München.

Heibert F. [1993], Das Wortspiel als Stilmittel und seine Übersetzung. Am Beispiel von sieben Übersetzungen des „,Ulysses “ von James Joyce, Tübingen.

Henley N.M. [1989], Körperstrategien. Geschlecht, Macht und nonverbale Kommunikation, Frankfurt a.M.

Hohenhaus P. [2000], Zur Verwendung humoristischen Materials im DaFUnterricht, in: Info DaF. H. 4, S. 433-448.

Holtbernd T. [2004], Humorzitien. Die etwas anderen Exerzitien, Münster. 
Howland Ch., Lentz M., Thoma D. [1999], Ganz Deutschland lacht. 50 deutsche Jahre im Spiegel ihrer Witze, München.

Höfner E., Schachtner H.-U. [2004], Das wäre doch gelacht! - Humor und Provokation in der Therapie, Hamburg.

Höfding H. [1930], Humor als Lebensgefühl, Leipzig.

Humorforschung. Wo ist der Witz?[2007], http://www.zeit.de/2007/31/humorforschung.

Humorstrategien. Das wäre doch gelacht, von http://www.schrotundkorn.de./ (Stand vom Mai 2012).

Hübner K. [2006], Mehrsprachigkeit und Identität. "He Alder, hassu Ei-Pott bei? “, Goethe-Institut, Die Macht der Sprache - Mehrsprachigkeit, online- Redaktion, Mai 2006, http://www.goethe.de/lhr/prj/mac/msp/de1398809.htm.

Interview mit Helga Kotthoff [2008], Die Humorforscherin: Lachen, theoretisch, http:// fudder.de/artikel/2008/01/21/die-humorforscherin-eher-sach-als-lachgeschichten/.

Janicka J. [2011], Sprachspiel - Wortspiel - Wortbildungsspiel, in: Kaczmarek D., Makowski J., Michoń M., Weigt Z. (Hrsg.), Felder der Sprache - Felder der Forschung. Lodzer Germanistikbeiträge, Łódź, S. 69-76

Kabaret Ani Mru-Mru [2006], Edycja specjalna, 2 x DVD, Lublin.

Kabaret PARANIENORMALNI [2009], „Mariolka prawdę Ci powie“, DVD, Warszawa.

Kaya Yanar erzählt: „Liebeserklärung an die Nation“, [2004], http://www.kino.de/news/ liebeserklaerung-an-die-nation/165950.

Körpersprache. Ein Lächeln wirkt Wunder [2007], http://www.focus.de/finanzen/karriere/ management/koerpersprache/koerpersprache/koerpersprache_aid_5470.html.

Kotthoff H. [1986], Scherzen und Lachen in Gesprächen von Frauen und Männern, in: Der Deutschunterricht, Nr. 3, S. 16-29.

Kotthoff H. [1988], Das Gelächter der Geschlechter. Humor und Macht in Gesprächen von Frauen und Männern, Frankfurt.

Kotthoff H. [1992], Von gackernden Hühnern und röhrenden Hirschen.Konversationelles Scherzen zwischen Männern und Frauen, in: Vogel T. (Hrsg.), Vom Lachen. Tübingen, S. 192-209.

Kotthoff H. [1994], Worte und ihre Werte. Konversationelle Stildifferenzen und Asymmetrie, in: Huber J., Müller A.M. (Hrsg.), Kultur und Gemeinsinn, Basel, S. 73-99.

Kotthoff H. [1996b], Conversational joking and impoliteness. On relational politics, in: Folia Linguistica 30, 3-4, S. 299-327.

Kotthoff H. [1997], Erzählstile von mündlichen Witzen. Zur Erzielung von Komikeffekten durch Dialoginszenierungen und die Stilisierung sozialer Typen im Witz, in: Selting M., Sandig B. (Hrsg.), Sprech-und Gesprächsstile, Berlin/New York, S. 123-170.

Kotthoff H. [1998a], Witzige Darbietungen auf eigene Kosten. Über Komplexitäten Weiblicher Imagepolitik in der Scherzkommunikation, in: Zeitschrift für germanistische Linguistik 139/140, S. 253-279.

Kotthoff H. [1998b], Spaß Verstehen. Zur Pragmatik von konversationellem Humor, Tübingen.

Kotthoff H. [1999], Coherent Keying in Conversational Humour: Contextualising Joint Fictionalisation, in: Bublitz W., Lenk U.,Ventola E. (Hrsg.), Coherence in Spoken and Written Discourse, Amsterdam, S. 125-150.

Kotthoff H. [2000], Konversationelle Parodie. Über komische Intertextualität in der Alltagskommunikation, in: Germanistische Linguistik 153, S. 159-186. 
Kotthoff H. [2002a], Über die Zähmung des weiblichen Gelächters.in: Chauré E., Gutknecht O., Schmidt C. (Hrsg.), Zur Konstruktion von Geschlecht in Sprache, Gesellschaft und Kultur. Gedächnisschrift für Gisela Schoenthal, Freiburg, S. 103-113.

Kotthoff H. [2002b], Ironie in Privatgesprächen und Fernsehdiskussionen. Zum Zusammenhang von Konversation, Kognition und Ethnographie, in: Keim I., Schütte W. (Hrsg.), Soziale Welten und kommunikative Stile. Festschrift für Werner Kallmeyer, Tübingen, S. 445-473.

Kotthoff H. [2002c], Humor und (Un)höflichkeit. Über konversationelle Beziehungspolitik, in: Felderer B., Macho T. (Hrsg.), Höflichkeit. Aktualität und Genese von Umgangsformen, München, S. 289-318.

Kotthoff H. [2003], Witz komm raus. Komik und Humor bei Kindern - ein Überblick,in: TelevIZIon. Internationales Zentralinstitut für das Jugend- und Bildungsfernsehen 16,1, S. 4-12.

Kotthoff H. [2004a], Lachkulturen heute: Humor in Gesprächen, http://opus.bsz-bw.de/ hdms/volltexte/2004/338.

Kotthoff H. [2004b], Geschlechterverhältnisse in der Scherzkommunikation: Althergebrachtes und neue Trends in der Alltags- und Fernsehkomik, in: Epp H. (Hrsg.), Gender studies - Interdisziplinäre Ansichten 1, Freiburg, S. 15-53.

Kotthoff H. [2004c], Overdoing culture. Sketch-Komik, Typeninstilisierung und Identitätskonstruktion bei Kaya Yanar, in: Hörning K., Reuter J. (Hrsg.), Doing culture. Neue Positionen zum Verhältnis von Kultur und sozialer Praxis, Bielefeld, S. 184-201.

Kotthoff H. [2006a], Lachen im Kontext von Selbstbewitzelung. Sequentielle Platzierung, Performanz und Funktion - gesprächsanalytisch betrachtet, in: Mauser W., Pfeiffer J. (Hrsg.), Lachen. Reihe Freiburger Literaturpsychologische Gespräche, Band 25, Freiburg, S. 57-75.

Kotthoff H. [2006b], Freud und sein weiblicher Witz, in: Fallend K. (Hrsg.), 100 Jahre Der Witz und seine Beziehung zum Unbewussten, Insbruck, S. 159-180.

Kotthoff H. [2007], Gemeinsame Herstellung humoristischer Fiktionen im Gespräch. Eine namenlose Sprechaktivität in der spielerischen Modalität, in: Andresen H., Januschek F. (Hrsg.), SpracheSpielen, Freiburg, S. 187-214.

Kotthoff H. [2009], Ethno-Comedy zwischen Unterlaufung und Bestätigung von Stereotypen. Potentiale für den Deutschunterricht, in: Nauwerck P. (Hrsg.), Kultur der Mehrsprachigkeit in Schule und Kindergarten, Freiburg, S. 37-55.

Kotthoff H. (Hrsg.) [2006], Scherzkommunikation. Beiträge aus der empirischen Gesprächsforschung, Radolfzell, http://www.verlag-gespraechforschung.de.

Kowalewska-Dąbrowska J. [2009], Humor w języku osób publicznych. Perspektywa odbiorcy, in: Dżereń-Głowacka S., Kwiatkowska A. (Hrsg.), Humor. Teorie-praktyka - zastosowania. Zrozumieć humor, Piotrków Trybunalski, S. 41-49.

Kucharski A. [2009], Struktura i treść jako wyznaczniki komizmu tekstów humorystycznych, Lublin.

Kunschmann D. [2003], Die besten Witze von A-Z, München

Kwiatkowska A., Stanecka A. (Hrsg.) [2012], Humor. Teorie - praktyka-zastosowania. Kody humoru, Piotrków Trybunalski.

Legman G. [1970], Der unanständige Witz, Hamburg.

Lew R. [2000], Dowcip językowy w świetle najnowszych badań językoznawczych teorii humoru, in: Gajda S., Brzozowska D. (Hrsg.), Świat humoru, Opole, S. 127-135. 
Lixfeld H. [1978a], Witz. Arbeitstexte für den Unterricht, Stuttgart.

Lixfeld H. [1978b], Witz und Aggression. Zur Begriffsbestimmung und Funktion einer Textsorte, in: Zeitschrift für Volkskunde, H. 74, S. 1-19.

Lixfeld H. [1984], Witz und soziale Wirklichkeit. Bemerkungen zur interdisziplinären Witzforschung, in: Fabula. Zeitschrift für Erzählforschung 25, S. 183-213.

Maletzke G. [1963], Psychologie der Massenkommunikation, Hamburg.

Marhenke D. [2003], Britischer Humor im interkulturellen Kontext, Braunschweig.

Marfurt B. [1977], Textsorte Witz. Möglichkeiten einer sprachwissenschaftlichen Textsortenbestimmung, Tübingen.

Matschnig M. (2007a), ,Ein Hauch von Nichts “ entscheidet, in: Weser - Kurier 5, http:// matschnig.com/de/images/pdf/WeserKurier.pdf.

Matschnig M. [2007b], Gesten sagen mehr als Worte. Die Körpersprache gibt den Ton an, in: telis magazin, S. 142-144, http://matschnig.com/files/TELIS.pdf.

Matschnig M. [2011a], Der Eindruck zählt: Was uns die Körpersprache sagt, In: Badische Zeitung vom 18.01.2011, http://www.badische-zeitung.de/liebe-familie/dereindruck-zaehlt-was-uns-die-koerpersprache-sagt--40096498.html.

Matschnig M. [2011b], Zuerst spricht der Körper, in: Kärtner Wirtschaft 10, http:// matschnig.com/de/images/MX-2600N_20111007_100213.pdf.

Matschnig M. [2012], Die Körpersprache der Macher, in: Ruhrzeitung, September, http:// matschnig.com/home/wp-content/uploads/2012/02/Koerpersprache.pdf.

Michalewski K. (Hrsg.) [2002], Tekst w mediach, Łódź.

Millowitsch W. [1970], Da bleibt kein Auge trocken. Die besten Witze gesammelt von Willy Millowitsch, München

Moser-Rath E. (1972/73), Galgenhumor wörtlich genommen, in: Schweizerisches Archiv für Volkskunde, Bd. 68/69, S. 423-432.

Mühlisch S. [1997], Mit dem Körper sprechen, Wiesbaden.

Mühlisch S. [2007], Fragen der KörperSprache, Paderborn.

Müller G. [1964], Theorie der Komik, Würzburg.

Müller G. [2002], Das thema tisch-Spiel, in: Sprachreport 18, H. 1, S. 14-17.

Neumayer, I. (2007), Interview mit Kaya Yanar ,Mein Bruder wurde katholisch unterrichtet, ich evangelisch ", in: GALORE 21, S. 155-161.

Pałuszyńska E. [2007], Strategie wywierania wpływu realizowane na poziomie struktur globalnych dialogu, in: Habrajska G., Obrębska A. (Hrsg.), Mechanizmy perswazji i manipulacji, Łódź, S. 251-259.

Pałuszyńska E. [2008], Gry językowe jako źródto humoru w felietonach radiowych,in: Dżereń-Głowacka S., Kwiatkowska A. (Hrsg.), Humor. Teorie - praktyka - zastosowania. Odcienie humoru, Piotrków Trybunalski, S. 39-45.

Plessner H. [1982], Lachen und Weinen. Eine Untersuchung nach den Grenzen menschlichen Verhaltens, in: Plessner H., Gesammelte Schriften VII, Frankfurt a. M., S. 201387.

Poethe H. [2002], Wort(bildungs)spiele, in: Barz I., Fix U., Lerchner G. (Hrsg.), Das Wort in Text und Wörterbuch, Stuttgart/Leipzig, S. 23-40.

Preisendanz W. [1970], Über den Witz, Konstanz.

Prestin E. [2000], Ironie in Printmedien, Wiesbaden. 
Quasthoff U. M. [1989], Ethnozentrische Verarbeitung von Informationen: Zur Ambivalenz der Funktion von Stereotypen in der interkulturellen Kommunikation, in: Matusche P. (Hrsg.): Wie verstehen wir Fremdes? Aspekte zur Klärung von Verstehensprozessen, München, S. 37-62.

Radomska A. [1994], Humor a płeć. Przegląd badań ze szczególnym uwzględnieniem okresu średniego i późnego dzieciństwa, in: Kwartalnik Polskiej Psychologii Rozwojowej 2, S. 16-31.

Radomska A. [2000], ,, Co kogo śmieszy?” - o różnicach indywidualnych w preferencjach komizmu u ludzi dorostych, in: Nowiny Psychologiczne 3, S. 73-97.

Radomska A. [2002], Rola poczucia humoru w procesie radzenia sobie ze stresem przegląd badań, in: Heszen-Niejodek I., Mateusiak J. (Hrsg.), Konteksty stresu psychologicznego, Katowice, S. 150-166.

Radomska A., Klinowska A. [2004], Czy Polaków i Niemców śmieszy to samo? - o narodowych różnicach w odbiorze komizmu, in: Psychologia Rozwojowa 5, S. 189-203.

Radomska A., Wiśniewska K. [2009], Przekraczając granice - humor feministyczny jako skuteczny oręż $w$ walce z seksizmem, in: Dżereń-Głowacka S., Kwiatkowska A. (Hrsg.), Humor. Teoria - praktyka - zastosowania. Zrozumieć humor, Piotrków Trybunalski, S. 299-315.

Radomska A., Jelińska, M. [2009], Społeczne funkcje humoru, in: Świątkiewicz W.,

Świątkiewicz-Mośny M., Ślęzak-Tazbir W. (Hrsg.), Oblicza humoru, Bd. III, Warszawa, S. 21-37.

Rojek E. [2003], Łaskotanie mózgu. Co wiemy o śmiechu i humorze, in: Kosmos 52, S. 237-247.

Raeithel G. [1996], Der ethnische Witz. Am Beispiel Nordamerikas, Frankfurt a.M.

Raskin V. [1985], Semantic Mechanism of Humour, Dordrecht.

Raskin V. (Hrsg.) [2008], The Primer of Humor Research, Berlin.

Röhrich L. [1977], Der Witz. Figuren, Formen, Funktionen, Stuttgart.

Rösch O. (Hrsg.) [2000], Stereotypisierung des Fremden. Auswirkungen in der Kommunikation, Berlin, S. 85-112.

Sauer N. [1998], Werbung - wenn Worte wirken. Ein Konzept der Perlukation, entwickelt an Werbeanzeigen, München/Berlin/Münster/New York.

Schippan T. [1972], Einführung in die Semasiologie, Leipzig

Schröder S. [2001], Interview mit Kaya Yanar „,Was willst du?!“, in: Subway Magazin, April.

Schubert G. [1999], Homo narrans und homo ridens in Südosteuropa. Alltagsbewältigung und Identität in Schwank und Witz, in: Die Welt der Slaven XLIV, S. 135-154.

Schweikle G., Schweikle I. [1990], Metzler-Literatur-Lexikon: Begriffe und Definitionen, Stuttgart.

Sikorska K. [2009], Zur Sprache der deutschen Comedyshow, in: Sadziński W. (Hrsg.), Acta Universitatis Lodziensis. Folia Germanica 5, Łódź, S. 29-37.

Sikorska K. [2009b], Język niemieckiego programu komediowego ,, Was guckst du??, in: Dżereń-Głowacka S., Kwiatkowska A. (Hrsg.), Humor. Teorie - praktyka-zastosowania. Zrozumieć humor, Piotrków Trybunalski, S. 161-167. 
Sikorska-Bujnowicz K. [2010], Polysemie und Homonymie als Mittel des Humoristischen in einem Sketchtext, in: Sadziński W., Sikorska-Bujnowicz K. (Hrsg.), Adäquatheit in Sprache und Literatur, Acta Universitatis Lodziensis. Folia Germanica 6, S. 69-78.

Sikorska-Bujnowicz K. [2011a], Sprachliche und außersprachliche Mittel des Humoristischen am Beispiel von „,Was guckst du? “, in: Kaczmarek D., Makowski J., Michoń M., Weigt Z. (Hrsg.), Felder der Sprache - Felder der Forschung, Łódź, S. 134-141.

Sikorska-Bujnowicz K. [2011b], Deutscher Pressewitz und seine gesellschaftskritische Funktion, in: Sadziński W. (Hrsg.), Die Presse als sprach- und kulturwissenschaftliches Forschungsobjekt, Acta Universitatis Lodziensis. Folia Germanica 7, S. 69-78.

Sikorska-Bujnowicz K. [2012a], ,Was guckst du? “ als ein multikulturelles Comedy-Programm im deutschen Fernsehen, in: Kaczmarek D., Makowski J., Michoń M., Weigt Z. (Hrsg.), Felder der Sprache - Felder der Forschung. Impulse für Forschung und Lehre, Łódź, S. 55-62.

Sikorska-Bujnowicz K. [2012b], Ethnowitz als Wiedererkennungszeichen der deutschen

Comedy-Sendung ,, Was guckst du? “, in: Sadziński W., Gołaszewski M. (Hrsg.), Wechselbeziehungen zwischen Sprache, Literatur und Kultur, Acta Universitatis Lodziensis. Folia Germanica 8, Ł, S. 17-24.

Sikorska-Bujnowicz [2012c], Polisemia i homofonia jako środki budowania humoru na przykładzie skeczów kabaretu „Paranienormalni”, in: Kwiatkowska A., Stanecka A. (Hrsg.), Humor. Teorie - praktyka - zastosowania. Kody humoru, Piotrków Trybunalski, S. 153-159.

Tęcza Z. [1997], Das Wortspiel in der Übersetzung. Stanisław Lems Spiele mit dem Wort als Gegenstand interlingualen Transfers, Tübingen.

Titze M. [2009a], Wie komisch ist der Humor? Über Erheiterung, Lachen, Schadenfreude, Inkongruenz und Ironie, in: TPS - Theorie und Praxis der Sozialpädagogik 6, S. 20-25, http://www.michael-titze.de/ (Stand vom Mai 2012).

Titze M. [2009b], Die disziplinarische Funktion der Schadenfreude - oder: Die Ambivalenz des Lachens, in: Wahl P., Sasse H. Lehmkuhl U. (Hrsg.), Freude - Jenseits von Ach und Weh?, Beiträge zur Individualpsychologie, Bd. 37, S. 11-39 (gekürzt), http://www.michael-titze.de/ (Stand vom Mai 2012).

Ulrich W. [1976], Wortbildungsspiele. Semantik des komplexen Wortes, in: Diskussion Deutsch 31, S. 424-437.

Ulrich W. [2007], „Wie vermehren sich Mönche und Nonnen? - Durch Zellteilung..."Wie und mit welcher Absicht spielt man mit der Sprache?, in: Der Sprachdienst 51, S. 189-200.

Wie man ehrliches Lächeln erkennt [2007], von: Körpersprache. Ein Lächeln wirkt Wunder, http://www.focus.de/finanzen/karriere/management/koerpersprache/koerpersprache/koerpersprache_aid_5470.html.

Wierzbicka A., [1999], Język-umyst - kultura, Warszawa.

„Was guckst du?”, (1995-2000), eigene Aufnahmensammlung der deutschen Fernsehsendung von SAT.1

http://www.witz-des-tages.de/

http://www.dein-witz.de/

http://www.grocceni.com/

http://www.lustich.de/ 


\section{ANHANG}

\section{Liste der besprochenen Witze ${ }^{73}$}

(1) Wie lautet die Werbung eines polnischen Reisebüros in Deutschland?

Kommen Sie nach Polen, Ihr Auto ist schon da!

(2) Kauf + ein Türke eine Villa, sein Nachbar auch. So ging es weiter mit anderen Sachen. Dann sagt der Türke: "Ich habe es besser." "Warum?", fragt der deutsche Nachbar, der Arzt ist. "Ich habe einen Arzt als Nachbar und Sie - einen Türken!"

(3) Der Schotte kommt in die Bäckerei und bittet den Bäcker „Bitte ein Stück Brot! Wickeln Sie es bitte in die Zeitung von heute ein!"

(4) Ein Russe bestellt immer fünf Schnaps gleichzeitig im Restaurant. Er trinkt für seine vier gefallenen Kameraden mit. Es soll eine Abmachung sein, dass der Überlebende für die anderen mittrinken müsse und er sei der einzige Überlebende. Eines Abends bestellt er nur vier Schnaps.

"Ich trinke nur für meine gefallenen Freunde. Ich selbst bin Antialkoholiker geworden."

${ }^{73}$ Als Textvorlagen werden hier die in diesem Buch präsentierten und analysierten Witze zusammengestellt. Sie entstammen den Internetseiten: http://www.witz-des-tages.de/, http://www. dein-witz.de/ und den Sammlungen von Witzen: Millowitsch [1970]; Kunschmann [2003],; Drews [2005; Hallstatt [2002]. 
(5) Die Frau eines Ostfriesen bekam Drillinge. Was macht der Ostfriese? Er sucht nach den anderen beiden Männern.

(6) Was machen die Ostfriesen bei Ebbe? Sie verkaufen Land an die Schwaben.

(7) Ein Polizist kommt in eine Buchhandlung und will einen Akkordeon kaufen.

Die Verkäuferin erwidert : „Es tut mir leid, aber es ist kein Akkordeon, es ist der Heizkörper!"

(8) Warum gehen die Polizisten nie alleine? Der eine kann lesen, der andere schreiben.

(9) Der Arzt beruhigt seinen Patienten vor der Operation. 2Machen Sie sich keine Sorgen. Ich habe diesen Eingriff schon hundertmal vorgenommen!" Der Patient sagt "Da haben Sie mich aber beruhigt, Herr Doktor!" Der Arzt erwidert "Na ja, einmal muss es doch klappen!"

(10) Jada dwie blondynki tramwajem. W pewnym momencie jedna z nich pyta "Dojadę do dworca?"

Na co druga: "A ja?"

(11) Was sagt die Blondine, wenn sie erfährt, dass sie schwanger ist? "Sind Sie sicher, Herr Doktor, dass es meins ist?"

(12) Ein Chinese kommt mit zwei Blondinen aus der Bäckerei. Warum? Er wollte zwei Blödchen!

(13) Zwei Blondinen treffen sich. Die eine prahlt: "Ich habe gestern einen Rekord geschlagen!" "Wieso?" fragt die andere "Ich habe ein Puzzle nur in 3 Wochen gemacht, obwohl auf der Packung geschrieben steht 3-5 Jahre!"

(15) Der Chef brüllt seinen neuen Mitarbeiter an: "Bringen Sie etwas zustande, was Hand und Fuß hat?" Der Buchhalter seufzt nur: "Nicht, solange Ihre Tochter die Pille nimmt!" 
(16) Eine Frau fragt den Verkäufer: „Kann ich die Bluse im Schaufensteranprobieren?" Der Verkäufer erwidert überrascht: "Sehr gern, tun Sie das, aber Sie können es auch in der Kabine tun"

(17) "Ist der Doktor zu Hause?" flüstert der Patient mit der Erkältungsstimme.

"Nein", haucht die hübsche Frau des Arztes, „kommen Sie doch schnell herein!"

(18) Die schottische Nationalmannschaft hat ein Spiel gewonnen.

Der Trainer kommt in die Kabine und sagt: „Jungs, ihr wart großartig. Ihr habt euch eine Erfrischung recht verdient. Los, John, mach das Fenster auf!"

(19) In der Zeitung: Schottisches Taxi stürzt in einen Fluss: 42 Tote...

(20) Woran erkennt man ein schottisches Schiff? Keine Möwen!...

(21) Wieso sind die Italiener so klein? Wenn sie groß sind, müssen sie arbeiten gehen.

(22) Ein Amerikaner und ein Italiener treffen sich in einer Kneipe. Sagt der Amerikaner: "Gib mir Deinen Hammer und ich bau Dir einen Flugzeugträger!"

Sagt der Italiener: „Gib mir Deine Tochter und ich mach Dir die Besatzung dazu!"

(23) Eine italienische Reisegruppe besichtigt die berühmten Niagarafälle.

Der Fremdenführer macht sie aufmerksam:" Meine Damen und Herren, wenn Sie für einen Moment Ihre Unterhaltung einstellen würden, könnten Sie das gewaltige Tosen und Brausen des Wasserfalls hören".

(24) Kommt ein Türke zum Arbeitsamt.

Berater: ... und was kann ich für Sie tun?

Ali: Ich brauche einen Job. 
Berater: Mann haben Sie Glück, sagt der Berater, ich hab sogar schon einen Job für Sie im Angebot.

Ali: Im ernst?

Berater: Ja, Sie werden eine große Luxuslimousine fahren, im Büro sitzen und 600.000 DM im Jahr verdienen. Ali: Sagen Sie mal, wollen Sie mich verarschen?

Berater: Na hören Sie mal, wer hat den damit angefangen?

(25) Ein Türke ist auf der Autobahn unterwegs. Plötzlich bekommt er einen Plattfuss. Als er gerade dabei war das Rad zu wechseln, kommt ein Pole angefahren steigt aus und geht zu dem Türken sein Auto und reist das Radio raus. Darauf der Türke wütend: " Ey, spinnst du!"

Da meinte der Pole: „Pssst, ich Radio und du Reifen."

(26) Welches ist das freundlichste Volk auf der Welt? Die Türken, sie kommen mit 20 Mann auf dich zu und fragen dich, ob du ein Problem hast.

(27) Ein Satz mit 10 Wörtern und 4 Lügen? Ein ehrlicher Pole fährt nüchtern mit seinem Wagen

(28) Was ist in Polen der Unterschied zwischen einer Hochzeit und einer Beerdigung? Auf letzterer ist einer weniger besoffen.

(29) In Polen hat man nie Probleme, wenn man im Halteverbot parkt: Bis die Politesse da ist, ist der Wagen eh geklaut.

(30) Surfen

Thomas geht zur Party. Fragt er die Partygäste: "Was machst du denn nächstes Wochenende?" - "Surfen" - „...und du?" - "Surfen” - "...und du?" "Auch surfen." Nach hunderten gleichen Antworten geht Thomas zufrieden nach Hause und eröffnet gleich am nächsten Tag einen Surfladen. In einem halben Jahr ist er Pleite. Auf der nächsten Party trifft er seine Freunde und wundert sich, dass keiner bei ihm eingekauft hat. Fragt sein Freund: "Na Thomas, hast du dir endlich auch einen Computer gekauft?" 
(31) Blondine am Computer

Warum schüttet eine Blondine Wasser über den Computer?

Damit sie auch mal surfen kann!

(32) Ofen

Sitzen eine Blondine und ihr Freund im Wohnzimmer. Sagt der Freund: "Kannst du mal bitte den Ofen anmachen?" Darauf die Blondine: "Na, du süßer, sexy kleiner Ofen..." Freund: „Nee, du sollst ihn bloß anfeuern!!" Ruft die Blondine: „O-FEN, O-FEN...!"

(33) Zebra

Ist eine Blondine im Zoo, sieht sie am Zaun vom Zebragehege ein Schild ,frisch gestrichen'. ,Oh!' sagt sie: ,ich dachte die Streifen wären echt!"

(34) Autounfall mit Blondine

Eine Blondine hat mit ihrem Auto ein anderes Fahrzeug gerammt. Brüllt der Fahrer: "Sie dummes Huhn, haben Sie überhaupt eine Fahrprüfung gemacht?" Zischt die Blondine zurück: "Bestimmt öfter als Sie!"

(35) Schwangerschaftstest

Zwei Blondinen unterhalten sich. Sagt die eine: "Ich machte einen Schwangerschaftstest".

Darauf die andere: „Und, waren die Fragen schwer?"

(36) Blondine und Fernseher

Warum streut eine Blondine Pfeffer auf ihren Fernseher? Damit das Bild schärfer wird!

(37) Co to jest - krzywi się w biurze i na nodze? Szef / szew.

(38) Was machen die Elefanten, wenn sie wissen wollen, wie spät es ist? Sie gehen zum Urwald!

(39) Woraus wird ein deutsches Auto gebaut? - Aus deutschem Stahl. Woraus wird ein schwedisches Auto gebaut? - Aus schwedischem Stahl.

Und woraus wird ein polnisches Auto gebaut? - Aus Diebstahl! 
(40) Was haben Robinson Crusoe und Beamte gemeinsam?Beide warten auf Freitag

(41) Kommt ein Frosch in einen Milchladen. "Was wünschen Sie?", fragt der Verkäufer "Quaaaaak!"

(42) Ein Mann besucht seinen sterbenden Freund. "Ich leide Höllenqualen", ächzt der und wirft sich auf seinem Lager herum. "Jetz† schon?" fragt der Freund

(43) "Wenn ich sterbe", sagt der Mann nachdenklich, "möchte ich verbrannt werden"

"Typisch für dich", sagt seine Frau gehässig. "Dann werde ich auch einmal die Asche im ganzen Haus verstreuen"

(44) "Stellen Sie sich vor", weint Frau Meier, "mein Mann ist von einer Dampfwalze überfahren worden und liegt im Städtischen Krankenhaus." "Grauenhaft", ruft die Freundin aus. "In welchem Zimmer liegt er denn?"

"Zimmer dreizehn bis achtzehn"

(45) "Herr Müller", ruft die Nachbarin aufgeregt, "Ihre Frau ist soeben im Dorfweiher ertrunken!" "Dann sollten Sie sofort die Seuchenstelle verständigen!"

(46) Der Maler sagt nachdenklich zu seinem Modell: "Ich überlege mir gerade, ob ich Sie nicht lieber nackt malen soll." Um Himmels willen", entsetzt sich das Modell, "bleiben Sie besser angezogen!"

(47) "Wissen Sie", wird der Zeuge gefragt, "ob der Angeklagte Selbstgespräche führt, wenn er allein ist?" "Ich war nie bei ihm, wenn er allein war" bedauert der Zeuge.

(48) Ein Vater will sein Kind von der Schule abholen. „Erwarten Sie ein Kind?" fragt eine Lehrerin

"Nein, ich bin einfach so dick!" erwidert der Vater 


\section{Verschriftungen der deutschen und polnischen Sketche ${ }^{74}$}

(14) Ein Polizist zu einem in Deutschland lebenden Türken: „Könnten Sie sich bitte ausweisen?" Der Türke antwortet: "Wieso sollte ich, ich lebe gerne in Deutschland!"

\section{(49) Advents- und Weihnachtszeit in Deutschland}

Am Sonntag ist schon ja erster Advent. Da rücken die Menschen wieder ein bisschen näher zusammen. Vor allem im Kaufhaus. "Können Sie bitte die Kerze aus meiner Nase nehmen???!!"

Für meine Familie waren diese Weihnachtvorbereitungen ja neu, als wir nach Deutschland kamen. Aber wir haben alles mal getestet Glühwein, Zimtsterne oder auch Marzipankartoffeln. Hier macht er eine kurze Pause, damit auf diese Weise noch Spannung steigen kann. Das war mein erster und letzter Kartoffelsalat! Er lächelt, das Lächeln ist echt, was wir an den Augen erkennen können, die mitlächeln. Anfangs haben wir sogar den ersten Advent mit dem ersten April verwechselt. Das machte uns dann Spaß - Scheiße in jeder Plätzchendose. Advent, Advent! Die Stimme lässt uns erkennen, wie glücklich er ist. Ich hab etwas anderes in der Dose. Schei... Beee!!! Jetzt erkennt man an seinem Gesichtsausdruck vor allem Überraschung und Wut.

Mittlerweile sind wir Ausländer aber auch voll auf Weihnachten eingestellt. Türken legen ihren Schlenker tiefer, im Verkauf statt Rosen - Tannenbäume, und Chinesen suchen sich ein Tier für ihren Weihnachtsbraten aus.

Hakan steht total auf diese Adventskränze mit Äpfeln und Walnüssen, obwohl er Walnüsse immer falsch versteht. "Was? Das sind Walnüsse? Du armer Fisch!"

Mein Kumpel Süleyman freut sich auf die Adventszeit. Der macht jede Nacht ein Türchen auf. Kaya macht ein Miene, die eine Überraschung ausdrückt, zieht die Augenbrauen hoch. "Oh, ein Rembrandt. Danke, Christkind!" Er macht Süleyman nach, lächelt zufrieden und glücklich.

Besonders liebe ich am Advent die Weihnachtsmärkte. Da kann man sich von seiner Nase leiten lassen. Wo es nach [...] Zimt riecht,

${ }^{74}$ Alle verschrifteten deutschen Texte (14) und (49-74) entsammen der eigenen Aufnahmensammlung der Comedy-Sendung „Was guckst du?” aus den Jahren 1995-2000, die polnischen dagegen sind Verschriftungen der Sketche von der Gruppe „Paranienormalni“ ( Kabaret PARANIENORMALNI (2009), „Mariolka prawdę Ci powie“, DVD, Wydawnictwo Agora S.A., Warszawa) und von „Ani Mru-Mru“ (Kabaret Ani Mru-Mru (2006), Edycja specjalna, 2xDVD, Wydawnictwo New Abra) 
gibt es Plätzchen und wo es nach Kotze riecht - den Glühwein! Ja, ein Glühweinstand. Glühwein. Am Glühweinstand merkt man immer - Besinnlichkeit und Besinnungslosigkeit liegen dicht beieinander.

Wir Ausländer finden den Advent immer toll. Zumindest anfangs. Endlich wieder Lichterketten. Hier macht er eine Pause, als ob er sich etwas überlegen würde. Sein Gesicht zeigt etwas Enttäuschung. Ach so, wegen Weihnachten und nicht wegen uns...

Der Advent ist natürlich auch die Zeit der Wunschzettel. Dabei geht es natürlich um die richtige Anrede. "Lieber Weihnachtsmann!..", nee, "Lieber Herr Weihnachtsmann!" nee, "Lieber sehr geehrter Herr Weihnachtsmann!" Scheiße!!! Oder... oder...die türkische Anrede Alde! oder "Sehr geehrter Herr Alde!"Bei meinem Vater hieß das nicht Weihnachtszettel, sondern Beate-Uhse-Katalog. Schickt mir dies und dies.Aber denken Sie dran, wichtig ist nicht, was man schenkt oder welche Marke oder wie teuer. Wichtig ist, dass man den Kassenbon aufbewahrt und wenn Sie dann mal einkaufen, sollen Sie sich besser nicht von irgendwelchen Meinungsforschern aufhalten lassen.

(50) "Achtung! (a) Wenn die Lampe nicht wäre, darf man nur mit der Ersatzlampe oder gleichen Typen Lampe wechseln. Bei dem Wechseln muss die Lampe nach gleicher Richtung in die Lampedose (b) zustecken sein. Wenn man die Lampe nicht wechseln könnte, kann man zum Beispiel (c) man mit dem Hersteller abreden $[\ldots]^{\prime \prime}$

K: Da fragt man sich, wie es zu solcher Übersetzung überhaupt kommen kann. Wir haben einen deutschen Übersetzer gefunden, der zu diesem Thema interessante Insider-Informationen liefern kann. Natürlich will er aus Angst vor seinen Kollegen unerkannt bleiben, deswegen befindet er sich in einem abgedunkelten Raum.

Guten Abend!

Guten Abend, Herr Yanar!

Sie behaupten also an der solchen Übersetzung steckt die regelrechte Übersetzungsmafia?

Nun, es ist folgendermaßen, dass...

Hallo, ich muss es kurz sauber machen.

Ja, danke für Informationen. Übrigens [...], die Lichterkette und viele andere Preise können Sie also beim Adventskalender gewinnen unter www.wasguckstdu.de [...] 


\section{(51) Hakan und Geburtsvorbereitung}

Im Studio von Yanar sitzen Aische und Hakan. Die Frau versucht Hakan dazu zu überreden, dass er bei der Geburtsvorbereitung dabei ist.

A: Hakan, gehst du jetzt zu der Geburtsvorbereitung oder ne, he? $\mathrm{H}$ : Hast du das Baby in den Ohren? Ich habe gesagt, ich muss Kayas Mütze finden.

A: Aaa!!!

$H:$ (a) Mach dir Knack am Arsch oder was?

A: Du Idiot, es ist das Baby, das Baby kommt. Könntest du mir helfen?

$H$ : (b) A, ruf mal an den Doktor Stefan Frank oder was! Scheiße!

A: Der Stuhl, der Stuhl, der Stuhl!!!

$H: J a, j a, j a$, der Stuhl, eine gute Idee mit dem Stuhl!

$A: \mathrm{Ja}, \mathrm{ja}$, der Stuhl!

$H$ : Und du?

A: Ey, (c) wie schon die Wehen, doch muss ich entspannen, o.k.?

Du musst mich jetzt massieren. Tu dies für Baby!

H: Da komm ich, komm jetzt dran..

A: Muss ich jetzt versuchen, das wegzuatmen.

H: Was? Das Baby?

A: (d) Den Schmerzen, du Idiot! Machst du mit?

$H:$ Ah, so..

A: Eee, mach du richtig!

H: Korrekt?

A: (e) Gehst du mal runte auf den Boden? ( $f$ ) Haltest du Hände auf den Knien

H: Scheiße!

A: Genau, jetzt mach mich den Becken locker..

$H$ : Das soll gut sein für das Baby?

A: Keine Ahnung, aber Mama macht Spaß!

$\mathrm{H}$ : Kommt das Baby jetzt raus oder nicht?

$A:$ Neee, $(g)$ aber hast du wenigstens gemacht Geburtsvorbereitung!

$H$ : E! Pass du auf! Dich verarsche ich auch, wenn ich schwanger bin oder so!

(52) Umfrage auf der Straße

D: Entschuldigung, wir machen eine Unterschriftenaktion zum Thema "Ausländer". Was sagen Sie zu den Ausländern? 
Der angehaltene Türke will sich aber nicht dazu äußern, sondern schnell weiter gehen.

T: Bitte, (a) müsse nach Hause gehen!

Der Deutsche ist mit der Antwort zufrieden, obwohl der Befragte etwas Anderes gemeint hat und lächelt.

D: Genau, müssen nach Hause gehen. Bitte unterschreiben Sie! Danke schön! Günther....

\section{(53) Türken und Polizeikontrolle}

Ein Auto wird von der Polizei angehalten. Am Steuer sitzt ein Türke, neben ihm - seine Frau. Der Polizist fragt den Fahrer nach dem Führerschein.

$P$ : Ihre Dokumente bitte.

T: E? Der Türke scheint die Frage nicht zu verstehen.

$\mathrm{P:}$ (a) $\mathrm{Na}$, Sie wissen schon..

T: (b) Nazis? Nazis?

P: Dokumente: Ausweis, (c)Führerschein...

$T:$ (d) Führer, Führer, Adolf Hitler! Hilfe!

P: Schon gut, schon gut, fahren Sie weiter! Der Polizist gibt auf und lässt sie los. Es stellt sich heraus, dass der Türke keinen Führerschein hat und seine mangelnden Deutschkenntnisse nur vortäuschte.

\section{(54) Ranjid und Kayas Mütze}

$\mathrm{R}: \mathrm{Na}$, Kaya, jetzt kriegst du ein Geschenk.

K: Ey! Was hast du in der Hand?

R: (a) Meine neue Putzlappe, habe ich es (b) in der Dusche gefunden, sehr (c) strapaziert. Wirklich! Der geht einfach nicht kaputt, der hat mehr Leben und wir haben schon 100!Gleich nur mal 99.

K: $O$, toll! Meine Mütze, gib her!

$R:$ (d)Du willst lieber nicht aufziehen...

K: Findest du? Ich wollte schon immer mal ohne Mütze...

R: Setz die auf!

K: Schon gut, schon gut!

(55) Ranjid im SAT.1-Studio
R: Hallo!
P: Hallo!
R: Kurz bevor wir anfangen - wer von euch ist Meister Proper und wer ist hier der General? 
P: Ich bin General und jetzt geht's los!

Ranjid geht von Tür zu Tür und nennt die bekannten Namen auf den Schildern.

R: Das ist Harald Schmidt, das ist Anke Angelke und der Ingolf Lück und die Benita.

Er fängt an zu putzen, gebraucht dabei die ihm zugeteilten Putzmittel.

R: Eins, zwei, drei und so..eins, zwei... ich habe es gleich benutzt und ein bisschen geputzt. So000... habe ich jetzt die Mattscheibe geputzt, jetzt ist es sauber.

P: Gut!

R: Es ist jetz† Klarscheibe!

Ranjid spielt jetzt in einer Quizshow und versucht auf die letzte Frage zu antworten.

P: Und jetzt die Meisterfrage - es geht um 16000 Mark. Passen Sie gut auf! Womit reinigt man am schnellsten? Erstens: mit Glasreiniger, zweitens - mit Spiritus, drittens - mit Spucke.

R: Mit Spucke?

P: Richtig! 16000 Mark!

\section{(56) Deutscher Integrationstest}

Szene in einem deutschen Büro. Ein Türke will den deutschen Pass bekommen und muss die drei letzten Fragen des Integrationstestes beantworten.

Beamter: Ja, Herr Egötürk, nur noch drei Fragen und Sie haben den deutschen Integrationstest bestanden. Im Hintergrund läuft die Musik aus , Wer wird Millionär?"

Welche Unterhose trug Helmut Schön bei der WM 1954 in der Schweiz beim Endspiel Deutschland - Ungarn, das wir mit 3:2 gewonnen haben?

Kandidat: Das ist eine Fangfrage. Erstens Trainer war (a) nichts Helmut Schön, sondern Sepp Herberger und zweitens (b) Unterhosenfarbe war blau.

B: Gut, gut, richtig. Im Hintergrund läuft die Musik aus ,, Wer wird Millionär?".

Aber wir haben noch zwei Fragen. Er guckt den Kandidaten ironisch und böse an. Was sagte August Heinrich Hoffmann von Fallersleben, der uns unsere Nationalhymne schenkte, am 26. August 1841 auf Helgoland um genau 19.45?

K: Das kann ich Ihnen genau sagen. Genau um 19.45 sagte er: "Wo habe ich diesen Liedtext hingetan?" Bei der Antwort gestikuliert er heftig. 
B: Gut, gut, schöooöon...Aber jetzt zur entscheidenden Frage. Dann können Sie Ihren deutschen Pass mit nach Hause nehmen. Was geschah in Deutschland von 1933 bis 1945?

K: Zwischen ' 33 und '45?

B: Der Beamte nickt.

$\mathrm{K}: A$, ich weiß, ich weiß. Er wird traurig, zuckt mit den Augenbrauen, verzieht das Gesicht zur Grimasse und überlegt sich die Antwort. Doch, doch, ich weiß!

B: Ja??????? Jetzt erkennt man an der Mimik, dass der Beamte völlig überrascht ist. Aber er hofft zugleich darauf, dass der Kandidat durchfällt.

K: Ich kann mich einfach nicht erinnern... Er ist sehr traurig und fängt an zu weinen, da er weiß, dass er die Chance verpasst hat.

B: Bravo!!!!!!! Ich auch nicht! Er macht die Begrüßungsgeste der Nazis. Herzlich willkommen in Deutschland! Sehr glücklich überreicht er dem Türken seinen erträumten deutschen Pass.

(57) K: Guckst du "Was guckst du?", ich freue mich sehr, dass "Was guckst du?" Fans in ganz Deutschland hat, z.B. in Kuxhafen, guckst du hier! Da habe ich Folgendes entdeckt Yanar zeigt das Kennzeichenschild cux-Du 33 und unter den Kleizuschauern wachsen sogar meine Nachfolger. Kaya zeigt die von den Zuschauern geschickte Bilder, auf denen u.a. Kinder mit einer schwarzen Baskenmütze zu sehen sind und ihn nachahmen.

\section{(58) Radio Süleyman}

S: Hallo, Leute! Hier ist wieder Sprechradio Süleyman. Natürlich (a) mit meine beste Freund Süleyhund. Scheiße! Muss mal wieder Gassi gehen. Braucht Auslauf. Also mach schnell. Erster Anrufer A1: Hallo. Süleyman! Ich bin traurig.

S: Traurig? Es ist ein Scheißname! (b) Aber was geht, traurig? A1: Nein, du verstehst mich nicht! Ich heiße Thomas und bin traurig. Ich lerne einfach keine Frau kennen, weil ich immer Angst habe, dass sie nur mein Geld wollen, ich bin nämlich sehr reich. S: Keine Angst, wenn du erst eine Frau hast, bist du bald nicht mehr reich...!

A1: Ich will keine Frau, die nur auf Materielles aus ist. Ich habe sogar schon einmal überlegt, mein Geld einfach wegzuschmeißen.

S: Was??? Mach keine Dummheiten, (c) helf ich dir, bist du alles los - Probleme und Geld. Scheiße!!! Süleyman ist aufgeregt, weil sich ein anderer Zuhörer dazwischen meldet und unterbricht das Gespräch 
mit Thomas. Des Weiteren unterhält sich Süleyman u.a. mit Wachtmeisterin Hümmer.

A3: Guten Tag, hier spricht die Wachtmeisterin Hümmer. Uns liegt eine Anzeige wegen Umweltverschmutzung vor.

S: Umweltverschmutzung? Ich bin doch kein Umweltverschmutzer. (d) Umweltverschmutzer ist Mann, der Sache wegwirft oder... Moment! Er kommt auf die Idee und verbindet sich wieder mit Thomas.

S: Traurig? Ich hab eine gute Idee für dich. Wirfst du doch Geld weg, packst du alles in großen Sack, wirfst du (e) rückwärts von Rheinbrücke..

A1: Was??? Von mir aus... Und dann lerne ich eine Frau kennen?

$\mathrm{S}: \mathrm{Ja},(f)$ vertraust du mir! Wieder verbindet er sich mit der Polizistin. Wachtmeisterin. (f) Habe ich gehört, steht Mann am Rheinbrücke und wirft Müllsack in Fluss. Das ist ein Umweltverschmutzer, darfst du ihn festnehmen, mich nicht!

Süleyman spricht wieder mit Thomas und arrangiert ein Treffen mit der Polizistin.

S: Thomas, (g) verspreche ich dich, wenn du den Sack geworfen hast, kommt nette Frau auf dich zu und nimmt dich mit, auch ohne Geld!

A1: Ohne? Danke, Süleyman! [...]

\section{(59) Passantenbefragung}

K: Entschuldigen Sie, der Herr, die Dame da, haben Sie vielleicht eine Minute Zeit für die Umfrage für eine türkische Zeitschrift? Eine Frage: warum, glauben Sie, vertragen die Deutschen mehr Bier als Ausländer? Zieht die Augenbrauen hoch und wartet auf die Antwort.

$P 1:$ Das ist regelmäßig, trinken!

K: Was gefällt Ihnen bei Deutschen am wenigsten? Mit drei Worten müssen Sie sagen. Also, eine..., ja?

P2: Sie sind pingelig

P3: Pingelig. Aber auch penibel, manchmal

P2: Ja, penibel, ja..und...

$\mathrm{K}$ : Was heißt penibel eigentlich? Weil ich kenne..Also...großzügig

P2. Nein, nein, penibel ist, ...ist...., ist...der Gegenteil von...

P3: Penibel ist pingelig

K: Ergänzen Sie: was für den Türken sein Schnurrbart ist, ist für den Deutschen sein... 
P1: Was würde ich sagen? Die deutsch.. typisch deutsch...kein Schnurbart.

K: Kaya nickt verständnisvoll und notiert die Antwort. Kein Schnurrbart Was heißt pingelig? Wenn man oft aufs Klo gehen muss und...?

P2: Nein, nein.. Doch, penibel ist Sauberkeit.

K: Aha, pingelig ist, wenn es nicht so sauber ist! Kaya nickt verständnisvoll und notiert die Antwort. Warum, glauben Sie, haben die deutschen Männer bei ausländischen Frauen so viel Erfolg? Also, bei uns, in der Türkei sind deutsche Männer sehr beliebt.

P1: Ehrlich?

$\mathrm{K}: \mathrm{Ja}$, glauben Sie mir.

P2: Das glaube ich nicht.

$\mathrm{K}$ : Glauben Sie nicht?

P2: Was ist denn mit verheirateten und und und..das weiß ich nicht $\mathrm{K}$ : Also, pingelig hat nichts zu tun mit aufs Klo gehen?

P1: Nein, nein, nein

P2: Nein. Es kommt eine Frau auf ihn zu und überrascht ihn mit dem Satz.

P4: Wissen Sie, wem Sie ähnlich aussehen?

K: Nein.

P4: Dem Komiker, der sagt „Was guckst du?" Kaya lächelt.

$\mathrm{K}: \mathrm{Ja}$, da gibt's einen. Kaya ist überrascht, lächelt. und gibt nicht zu wissen, dass es um ihn geht.

P4: Sie sind inm ähnlich.

$\mathrm{K}$ : Momentan, wir sind vom türkischen Schlag alle gleich!

P4: Ja, aber gut! Kaya ist überrascht, lächelt und gibt nicht zu wissen, dass es um ihn geht.

$\mathrm{K}: \mathrm{O}$, finden Sie ihn gut?

P4: Ja! [...]

\section{(60) Szene im Zimmer von Kaya Yanar}

Kaya unterhält sich mit Francesco

Francesco: Was ist so wichtig, (a) dass du mich hat wegholen von einem Essen mit (aa) bella signorina?

Kaya: (b) Du bist bestimmt wieder in die Hose gegangen.

Francesco: (c) Stimmt es nix! Bin ich gar nicht in die Hose gegangen, aber bis Bluse...

Kaya: Hör zu, ich habe der junge Damen eingeladen, die an einer Show mit Herzblatt spielen werden.

Francesco: Ich darf in deiner Sendung spielen mit drei jungen Damen? (d) Was ist genau für eine Sendung, die du hie machst? 
Kaya: Nein, nein! Du solltest dir nur eine aussuchen. Hier! Ich habe dir Fragen schon völlig aufgeschrieben. Bis später.

Francesco: Kandidatin eins, ich bin Italiener. Kannst du ein bisschen Italienisch? Wieso Italienisch??? (e) Besser ist es, viel Französisch...

\section{(61) Szene auf dem Polizeirevier}

Ein Pole, namens Olszewski, will eine Anzeige erstatten, denn jemand hat ihm sein Auto geklaut.

Polizist 1: Ihr Name?

Pole: Olszewski

Polizist 1: Nationalität?

Pole: Pole

Polizist 1: Und? Warum möchten Sie die Anzeige erstatten?

Pole: (a) Hat man mir geklaut meine Auto.

Polizist 1: Hehe

Pole: (b) Was los?

Polizist 1: Heheh, Tschuldigung, es ist nur, ein Pole, dem man das Auto geklaut hat. Egal. Haben Sie schon irgendwelche Hinweise auf den Täter?

Pole: Nein.

Polizist 1: Haben Sie schon bei mal bei Ihren Bekannten in Polen nachgefragt? Vielleicht haben die ja, naja. Waren denn im Wagen noch irgendwelche persönlichen Besitztümer?

Pole: Persönliche was??

Polizist 1: Naja, Sachen, die Ihnen gehören. Tasche, Hut, ... ein Brecheisen

Pole: Jetzt habe ich aber genug. Willst du damit sagen, dass (46c) jede Pole ist eine Dieb?

Polizist 1: Schon gut, tut mir leid. Die dummen Vorurteile, Sie wissen schon. Hier. Sie müssen noch einmal unterschreiben

Pole: Also (sucht sich ab, hat keinen Kugelschreiber)

Polizist 1: Nehmen Sie meinen. (Olszewski unterschreibt und verschwindet)

Polizist 2: Was?!

Polizist 1: Ah, nichts. Bin noch mal kurz weg. Mir n neuen Kuli kaufen. Wo ist mein Portemonnaie? Und meine Schreibmaschine? Heinz, hast du?

Polizist 2: Nee. (sitzt nackt am Schreibtisch) 
(62) Szene im "Radio Süleyman"

Süleyman: Hallo, Leute! Ich bins wieder, Süleyman. Heute (a) aus provisorische Studio, (b) naturlich mit meine beste Freund Süleyhund. Süleyhund, (c) kommst du! So, erster Anrufer. (d) Was gibt's oder gibt's nix

\section{(63) Ein Ausländer befragt die Passanten}

Kaya (K) steht, ein Wörterbuch in der Hand haltend, auf der Straße und fragt die Passanten (P1, P2) nach verschiedenen Sachen.

Kaya: (a) Entschuldigen, ich habe eine Frage..

K: (b) Wo... kann man... hier gut... Er fängt an, im Wörterbuch nachzuschauen.

P1: Langsam...

K: ...(c) Popel essen?

Das Gespräch wird unterbrochen und wir sehen den zweiten Fußgänger, der gefragt wird.

$\mathrm{K}:$ (d) Wo ist hier... die Toilette... machen?

P2: (e) Machen nichts. Machen nichts.

$\mathrm{K}$ : Doch, doch!

P2: Sie müssen zur Toilette.

$\mathrm{K}$ : Hose

P2: (f) Wenn Sie nicht rechtzeitig auf die Toilette, dann geht es in die Hose.

Eine vorbeigehende Frau wird gefragt.

K: (g) Entschuldigen. (h) Wo... kann man hier in den Rhein schiffen?

Sie ist überrascht, geht weiter, ohne ein Wort zu sagen.

An dieser Stelle wird der Dialog mit zwei früher erwähnten Passanten fortgesetzt.

P1: Da kommt unten aus, aus (der Mann gestikuliert viel) eine Säule (am Regenschirm will er dem Ausländer zeigen, was eine Säule ist und macht dabei eine mehrdeutige Geste, die mit der Selbstbefriedigung der Männer assoziiert wird. Kaya lacht.)

P1: Ja, Hohe Straße, ja... (schaut den Kaya an, um sich zu vergewissern, dass er ihn verstanden hat).

K: Säule (Kaya wiederholt die vorher von dem Passanten gemachte Geste), essen, (i) nichts Säule (lacht weiter) An dieser Stelle wird der Dialog mit dem netten älteren Mann fortgesetzt.

P2: Da ist die Toilette.

K: (j) Dort Hose 
P2: Da ist die Toilette.

K: O.K, danke schön!!

\section{(64) Türkischer Dolmetscher beim Bewerbungsgespräch}

Wir sehen einen Deutschen im Büro sitzen. Er ist Berufsberater (B). Zwei Leute kommen herein (T1 als Dolmetscher und T2 als Arbeitsuchender, Herr Elgül)

B: Herein! A, Herr Elgül... und wer sind Sie?

T1: Sein Bekannter. Ich übersetze.

B: Ah, ja. Es soll diesmal besser gehen. Nehmen Sie Platz.

T1: Danke schön.

B: O.K., dann übersetzen Sie mal, bitte. Guten Tag, Herr Elgül!

T1: (a) Hey, Alde! Sie begrüßen sich mit den Gesten wie die Mitglieder einer Subkulturgruppe.

B: Sie arbeiten seit 14 Jahren als Schlosser.

T1: (b) Ey, bist du Schlosser oder was? Er verzieht das Gesicht.

T2: Das weißt du doch!

T1: Ja.

B: und plötzlich hat Ihnen Ihr Arbeitgeber gekündigt

T1: (c) und dann haben sie dich die Schweine den Arschtritt vor die Tür gejagt

T2: (d) Dann sag doch, die Arschlöcher, ja!

T1: Ja.

B: Aus welchem Grunde ist das passiert?

T1: Wieso?

T2: (e) Ich habe Bohrmaschine geklaut.

T1: Herr Elgül und seine Arbeitgeber hatten gewisse Differenzen bezüglich seines Gehaltes und da seine Arbeitgeber auf wiederholte freundliche Schreiben von Herrn Elgül verständnislos, um nicht zu sagen, schroff reagierten, hat Herr Elgül, obwohl er eine sehr große Stütze für seinen Betrieb ist, ...war, ein weinig zu viel Eigeninitiative entwickelt. Das gefiel seinen Arbeitgebern überhaupt nicht und sie haben ihm deswegen gekündigt.

$B: H m m$, verstehe.

T1-T2: (f) Korrekt! Sie klatschen einander ab.

$B:$ Ja..., leider habe ich im Moment keine Angebote für Schlosser, aber können Sie sich vorstellen, als Packer zu arbeiten?

T1: (g) Ey, willst du Scheißjob als Packer oder was?

T2: Wie viel? Mit dem Reiben der Finger der rechten Hand wird von ihm auf das Geld hingedeutet. 
T1: Wie sind dann die Verdienstmöglichkeiten?

B: $O$, da vertut man sich. Moment... Ja... Er blättert die Unterlagen durch. Wenn Sie die Zulagen, Weihnachtsgeld und Wochenendausgleich berücksichtigen, dann kommen Sie auf 4500 brutto.

T1: (h)Sie wollen dich verarschen. (i) Das ist Scheiß-dreckarsch-scheißjob, keine Kohle, Dreckkohle, Scheiße.

T2: Er fängt an, auf Türkisch zu schimpfen und verlässt den Raum.

B: Was ist denn jetzt passiert? Was haben Sie gesagt?

T1: Ich habe natürlich nur das übersetzt, was Sie gesagt haben. Aber meine andere Frage: kann ich den Job haben?

$\mathrm{B}: \mathrm{Ja}$, natürlich.

\section{(65) Werbung für Dreier-Fahrt}

Wir sehen Hakan, der aus seinen langen Haaren eine merkwürdige, aber doch perfekt haltende Frisur gemacht hat.

20:00 Szeneladen,

Hakan: (a) Ey du, du kommst hier nicht rein! Die Frisur hält.

23:00 Discoeinlass,

Hakan: (b) Ey du, du kommst hier nicht rein! Die Frisur hält. 1:00 Coctailbar,

Hakan: (c) Ey du, du kommst hier nicht rein!

Perfekter Halt dank Dreier-Fahrt, denn nur die Dreier-Fahrt gibt Ihrer Frisur den perfekten Halt für den ganzen Tag.

\section{(66) Polizeikontrolle 1}

Ein Türke geht einem Polizisten entgegen. Wenn sie schon aneinander vorbeigehen, fragt der Polizist

P: Können Sie (a) sich bitte ausweisen?

Darauf antwortet der Türke

T: Wieso sollte ich? Ich lebe gerne in Deutschland.

\section{(67) Fahrschule Kelal Yildirim 4}

Fahrlehrer Yildirim spricht mit seinem deutschen Schüler Manfred Winter

Yildirim: Wenn du willst Auto fahren lernen wie ein Türke, du musst anfangen türkisch zu denken. Sonst (a) kriegst du Durchfall. 
(68) Francesco im Restaurant1

$F$ : $O$, Bella, ich habe noch nie so eine Frau gesehen,

$\mathrm{B}: \mathrm{Ja}$ ?

F:... die so schnell essen kann er dreht seinen Kopf zur Seite......und wiederholt diese Kopfbewegung ...... so viel Es sieht so aus, das er sich umsieht, ob jemand sie dabei beobachtet... und so teuer.

\section{(69) Francesco im Restaurant 2}

$\mathrm{F}$ : $O$, Bella, kannst du mir sagen, was ist das Parfüm, das du benutzt? Francesco zieht die Augenbrauen hoch. Die Frau nimmt die Frage als zeichen seines großen Interesses an ihr.

B: Wieso? Gefällt es dir? Die Frau ist überglücklich, dass er eben diesen Duft spürt.

F: No, no, no! Es überdeck† deinen Mundgeruch! Die Frau ist empört, steht auf und geht weg. Zahlen!

\section{(70) Francesco im Restaurant 3}

B: Zu dir oder zu mir? Die Frau lächelt verlockend.

F: Lieber zu mir. Von seinem Gesicht lässt sich aber keine Freude ablesen, eher das, dass gleich etwas Überraschendes gesagt wird.

B: Wieso? Die Frau erhöht die Stimme, zieht die Augenbrauen hoch, aber hört nicht auf zu lächeln. Man sieht ihre Überraschung wegen des Wortes lieber

F: Der Gesichtsausdruck von Francesco verrät böse Absichten - er lächelt seltsam, verzieht das Gesicht. Wegen meines Rasierapparates! Sie steht auf und geht weg.

\section{(71) Francesco im Restaurant 4}

$F$ : $O$, bella! Ich suche eine Frau, die nicht nur kochen kann..

B: Ja? Die Frau lässt sich auf dieses provokante Frage-Antwort-Spiel ein. Von ihrem Gesicht kann man ablesen, dass sie auf einen Heiratsantrag von Francesco wartet.

F: Sondern auch ... backen! Der Gesichtsausdruck ändert sich, die Frau ist empört, steht auf und verlässt das Restaurant. Zahlen! Francesco scheint aber zufrieden zu sein, zieht seine Augenbrauen hoch, bewegt seinen Schnurrbart, hebt die Hand hoch und ruft zum Kellner Zahlen! 
(72) Tarek ab del Kalek auf der Modemesse in Düsseldorf 1.

Tarek ab del Kalek spricht mit einem Model.

T: You speak Deutsch? Allemange, anything you come, spanish? How is it to play in Germania, to make the fashion-show in the..., here?

$M$ : Its good!

$T$ : Is good?

M: Jeah!

$T:$ Is ist different from Spain?

$M$ : I' ve never modelled in Spain

T: Are you from Spain?

$M:$ No, Kolumbien.

$T:$ Oh, Kolumbien! How is it to model in Kolumbien?

$M: I^{\prime}$ ve never modeled in Kolumbien.

$\mathrm{T}$ : Are you model?

M: Yes!

(73) Tarek ab del Kalek auf der Modemesse in Düsseldorf 2.

Tarek ab del Kalek präsentiert die Ausstellung von der Firma Kwark

Tarek: Auf dieser Messe sind Firmen aus der ganzen Welt vertreten. Dahinten Frankreich, hier Italien. Nur Dubai ist nicht (a) dubei, dabei, duba, duba, du, dabei.

Tarek: (b) Ist das hier alles Quark / Kwark?

Vertreter: Ja, das ist..

Tarek: (c) Alles Quark. Also Frauen ziehen gern Quark an. (d) Und für Männer ist es wahrscheinlich Quark, oder?

\section{(74) Schachweltmeisterschaften}

Am Tisch sitzen zwei Spieler, wobei der eine schwarz ist.

Richter: Ich begrüße die beiden Spieler im Finale der Schachweltmeisterschaft und eröffne damit offiziell die Partie. (a) Weiß beginnt

Spieler: He? Wer jetzt? 


\section{(75) $M$ jak młotek ${ }^{75}$}

Im Hintergrund hören wir die Musik aus dem Film „,M jak miłość“, der Titelsong hat jedoch einen geänderten Text (,, M jak mtotek wiele funkcji ma, M jak młotek zawsze radę da...). Der Hammer erscheint auf der Bühne und sucht nach dem Nagel, fragt dabei das Publikum, ob jemand ihn gesehen hat.

M: Gwoździu, gwoździu! Słuchajcie, nie widzieliście może gwoździa? Co, nie bardzo? Taki (1) przybity troche. $O$, jesteś..

G: O kurcze, czekaj, ale (2) mnie boli szyjka od obrazka. Er bewegt mit dem Kopf und man sieht, dass ihm etwas weh tut.

M: No gdzież ty się podziewał?

G: (3) Tkwiło sie to tu, to tam., nie? A co nowego na budowie?

$M$ : Co na budowie? (4) teb mnie strasznie boli. Er fasst sich am Kopf. Wiesz, słuchaj, jak ja się wczoraj (5) nawaliłem z kilofem, tak żeśmy (6) się skuli, słuchaj, no, impreza w "Castoramie" była, słuchaj, co mówie, no. Powiem ci, że nawet nie wiedziałem, że (7a) francuz potrafi (7b) takie śruby kręcić, słuchaj, no...

G: Męska impreza, co?

M: Nie była taka męska, wiesz, (8a) podkowa też tam była

$G$ : A ona po co?

M: (8b) Na szczęście, hyhyhy. Nie, no za kierowce robiła, wiesz.

G: Ty ty ty ty ty ty, a tej, no, wiertarki, nie było z wami?

M: Wiertarki? No co ty! Jaja sobie robisz? Nie słyszałeś o wiertarce? Er ist empört und überrascht, dass der Gesprächspartner von nichts weiß.

G: Nie.

M: (9) Wiertarka na słońcu leżała i udaru dostała. [...]

G: Ty patrz, jakie nieszczęście, co nie? Bo o betonie to styszałeś, prawda?

M: ... No gadaj, co z betonem? Geste und Mimik betonen die Wichtigkeit dieser Information.

G: (10) Wylali go!

$M$; [...] A o tapecie i ścianie styszałeś?[...] (11) Tapeta odeszła od ściany.[...]

(12) Przestało się między nimi kleić i (13) tapeta się zwinęła.

G: [...] Przecież (14) ściana zawsze tak była za tapeta.[...]

M: Ale znasz tapete, wiesz, jak (15) ona lubi się drzeć... Er zuckt mit den Schultern.

${ }^{75}$ Sketch von der Gruppe „Paranienormalni“ 
G: (16) A ściany maja uszy! [...] A powiedz, (17a) jak ściana to zniosła?

M: [...] Bardzo dobrze, przecież to (17b) ściana nośna. [...] Trzeba przyznać, że (18a) chociaż beton to (18b) ma kontakty. [...] Mit der Mimik wird die Begeisterung zum Ausdruck gebracht.

\section{(76) Mariolka $1^{76}$}

Auf der Bühne erscheint der als Frau verkleidete Igor Kwiatkowski, der in diesem Sketch die Figur Mariolka spielt. Mit einer Blondperücke, einen Pullover in grellen Farben anhabend, mit einer kleinen umgehängten Tasche. Wenn die Gestalt auf der Bühne erscheint, hören wir die Musik von Danzel (,,Don 't you know, pump it up, You got to pump it up, Don 't you know, pump it up, You got to pump it up“).Dieses Musikmotiv begleitet Mariolka in jedem Sketch und wird somit zum Wiedererkennungszeichen dieser Figur.

Słuchajcie, (19) ja pitole, (20) jestem taka podjarana, taka podenerwowana, że szok, normalnie, (21) kuwa, tipsy zjadłam, no mówię wam, (22) tera mi sie plastikiem odbija. Mam takiego (23) newsa dla Gabryśki, że jak jej powiem, to (24) kuwa, padnie. Greift zum Handy und versucht Gabryśka zu erreichen. Super, (25) kuwa, granie na czekanie! Man hört die Musik „Don't you know, pump it up, You got to pump it up, Don't you know, pump it up, You got to pump it up "No cześć, Gabryśka, Mariola mówi. A ty kto (26) kuwa, jesteś, a kim pan jest? Ale ja (27) kuwa nic nie rozumiem po polsku, jaki (28) „the subscriber you hale called"? [...] (29) Kuwa ${ }_{\perp}$ co to było? (30) Kuwa, ale (31) jestem pojechana. Próbuje raz jeszcze połaczyć się z Gabryśka. No cześć, no! Teraz dobrze, Gabryśka, słuchaj, mam dla ciebie takiego (32) newsa, że padniesz, dziewczyno, Mariolka ist nervös und läuft auf der Bühne hin und her (33) ja pitole, stoisz, to (34) kuwa usiadź, siedzisz, to (35) kuwa wstań, Gabryśka, twoja przyjaciółka (36) sensu stricto, czyli ja, (37) kuwa, wygrałam zagraniczna wycieczke, słuchaj, no gdzie jedziemy, gdzie się (38) kuwa bawimy, no nieważne, taki (39a) casting był "Zostań twarza firmy budowlanej" i (40) kuwa wygrałam. (39b) Co będe reklamować? Pustaki! No, (41) spoksik, spoksik.Gabryśka, no to gdzie się bawimy, gdzie szalejemy [...] Gabryśka, (42) gdzie jedziemy, gdzie się (43) kuwa bawimy? No, ja słyszałam, że jak sie jedzie na zagraniczna wycieczke, to się koniecznie trzeba (44) sczepić. No, ja wiem, że głupio, ty będziesz

\footnotetext{
${ }^{76}$ Sketch von der Gruppe „Paranienormalni“
} 
chciała gdzieś pójść, to jak pójdziemy takie sczepione? No, no. Mit der Mimik und den Gestem gibt sie ein Zeichen, dass sie derselben Meinung ist. Co? (45a) Paszport mam, a po co mam go (45b) przedłużać? Żeby mi się do torebki nie zmieścił? Greift zu ihrer Tasche und zeigt, wie groß diese ist. Ty, zabiore ten krem, co mi dałaś. No dziewczyno, (46) rewelka, (47) zarabisty jest, ale mi pudełka nie dałaś, nie wiem, na co on jest, co on robi. No, weź mnie (48) kuwa nie strasz, (49) ściaga pory??? Mit Angst greift sie an ihre Hose. Szczera jesteś, wiesz? Dobra, Gabryśka, [...] (50) gdzie jedziemy, gdzie się (51) kuwa bawimy? Co? (52a) Morze Egejskie? No wariatko, (52b) kuwa ${ }_{4}$ zwariowałaś? Dwa tygodnie z (52c) egejami? (54) Hello! Sie ist empört. Man sieht es ihr an. Ty, (55a) kuwa., gdzie ty, zaraz mnie, (55b) kuwa na wyspę Lesbos wyślesz! I co byśmy tam w ogóle robiły? Skoki na (56) bungee? Super. A ty wariatko, (57) kuwa_widziałaś, jakie to małe jest, no ja widziałam gościa w telewizji, jak na tym (58) bungee grat, no! Ahmt einen Banjospieler nach. Torebke (59) se kupiłam, (60) podrabiana imitacja sztucznej skóry. Słuchaj, okazyjnie, w tym sklepie "Wszystko po 5 złotych". Jak to (61) kuwa, ile dałam? Gabryśka, (62) kuwa, sklep po 5 złotych. (63) Don't matter! [...] A tak w ogóle, to co byśmy tam [na Hawajach] robiły? W czym spały? W (64) bunga bungalowach? A coś ty, ja tam tradycyjnie, jak ciepło jest, w samych gaciach wolę. A w ogóle słyszałam, że na Hawaje się (65) zarabiście długo pociagiem jedzie. Samolotem to (66) kuwa zapomnij, zapomnij, wiesz, jak ja (67) kuwa gadać lubię, a słyszałam, że tam na lotnisku jest taka (68) strefa bez słowa. Strefa bez słowa, mówię ci. [...] Dobra, Gabryśka, dawaj, gdzie jedziemy? Jamajka odpada, nie, nie, nie, tam sa te (69) laleczki łubudu, to odpada. [...] Ciebie jak o coś poprosić, to tak jak z tym calgonem, tak żeś mi poradziła. Co z (70) calgonem? Jeszcze się (70a) kuwa pytasz, beznadzieja! Nie dość, że (70b) kamienia nie usuwa, to wiesz, (70c) kuwa, jaka zgage miałam!

\section{(77) Mariolka $2^{77}$}

Auf der Bühne erscheint wieder der als Frau verkleidete Igor Kwiatkowski und wir hören die Musik von Danzel (,,Don't you know, pump it up, You got to pump it up, Don't you know, pump it up, You got to pump it up")

${ }^{77}$ Sketch von der Gruppe „Paranienormalni“ 
(71) Hejka! Cześć! Czekajcie, czekaj, telefon mam! Poczekajcie, Gabryśka dzwoni, poczekajcie... Gabryśka cześć! Hej Gabrycha. Gabryśka, skad Ty (72) kuwa wiedziałaś, że ja tu bede ? Nieważne. Gabryśka, czekaj, Gabryśka! Ty masz dzisiaj urodziny, (73) co nie? No to Gabryśka! (74) Happy Birthday kuwa to you! Happy Birthday kuwa to you! (75) Brechtasz się? No ja też! Sie lacht. Co robisz? (76) Bez kitu! Uczysz się? Ja (77) pitole! Naprawdę? Gabryśka, to ja słyszałam, że jak się człowiek dużo uczy, to musi dużo (78) magnezu jeść, masz magnez? Jak nie masz, widziałam, na lodówce masz, no! Sie lacht. Tylko tego plastiku (79) kuwa nie jedz, nie? A słuchaj, (80) a propo w ogóle nauki na uczelni, to muszę ci się pochwalić, że (81) założyłam kółko na uczelni, wiesz? No, metalowe, na głowe, (82) kuwa ściagnać nie mogłam! No mówię cil Nie wiem, sylwestra tu maja, czy coś, szaleja nie... Ale w ogóle, Gabryśka, smutna mam nowine, słuchaj, nie jest już hostessa w supermarkecie, wiesz... Dlaczego, dlaczego, nie wiem dlaczego, wiesz... Byłam na spożywczym, swoja osoba jedzenie reklamowałam, pomyślałam (83) se wiesz, nie wyłożę na tace, ludzie nie spróbuja. (84) kuwa nie kupia, co nie? Ty, wyłożyłam (85) zarabiście wszystkim smakowało, Ty, nie? Przyszła kierowniczka, zobaczyła to, z geba na mnie, wywaliła mnie, wiesz? A (86) zarabiste jedzenie w takich fajnych puszkach z pieskiem i kotkiem, no mówię ci, no. Sie lacht. A co, co, co, ty nie lepsza byłaś! Nie pamiętasz za co ciebie wywalili? No za to mleko, (87) helot, no... No jakie mleko, nie pamiętasz, co wszystkich mlekiem częstowałaś, a to sie okazało, że to mleczko do demakijażu było! Sie lacht. (88) Ja pitole, ja nigdy (89) tyle pawi na raz nie widziałam, normalnie szok... Sie lacht. Gabryśka, ale się w ogóle tym nie przejmuje, wiesz. Mam nowa robote, wiesz? W solarium (90) bede robiła, mówie ci. (91) Zarabiste, takie dużo, yyy imienia yyy...Andrzeja Leppera! Sie lacht. Gabryśka, (92) bez kitu, ty nie wiesz kto to jest Lepper? (93) Ja pitole, (94) jaka siara, dziewczyna...! (95) Kuwa, ja nie moge, nie wiesz, kto to jest Lepper? Gabryśka, to piłkarz jest, co ty! Ale słuchaj ty, poszłam do tego szefa na (96) rozmowe kwa... kwa... kwalifikacyjna, o! Ty i go pytam, (97) ile miesięcznie można wyciagnać, a on mówi, że 1500 , myślę (98) spoko pieniadz, co nie? To się go pytam, kiedy moge przyjść, a on mówi (99) "„powiem pani ten sposób - w ogóle tu pani nie musi przychodzić"! (100) Czujesz to?! (101) Bede miała 1500, w ogóle do roboty nie musze przychodzić! A Gabrycha! Gabrycha, mam nowego chłopaka. (102) Zarabisty, (103) rewelacja, niebieskooki blondyn, dociera to (104) 
kuwa do ciebie? (105) Czujesz to? (106) Rewelacja, słuchaj, przystojny, namiętny... Jak ma na imię? Gabryśka, (107) heloł, heloł! Przecież ja mam jakieś zasady, na pierwszej randceo takie rzeczy nie pytam, co nie? Co ty... Tylko żeśmy się całowali, wiesz. Ale słuchaj, zabiera mnie na romantyczna kolacje... do McDonald'a! Sie lacht. O (108) kuwa Gabryśka, (109) o kuwa, Gabryśka ty, (110) co to takiego jest przypomnij mi, ty, jak boli głowa i obrazki sa? Jak boli głowa i obrazki sa co to jest? Pomysł, właśnie, pomysł mam, ty! Słuchaj no, zrobię mu ten nasz test, pamiętasz? Jak kupi (111) cheeseburgera to sknera, co nie? Jak cały zestaw, znaczy, że ma gest, nie? Jak (112) Happy Meal, znaczy, że rodzinny jest, dzieci lubi, wiadomo (113) o co kaman, (114) co nie...Ty, a jak (115) wieśmaka, to wiadomo! Kupuję wieśmaka - zmieniam chłopaka, nie! Sie lacht. A Gabryśka... Gabryśka, pamiętasz tego pieprzyka co go miałam? No (116) co na niego tak chłopaki leciały... No co mnie z ta wokalistka mylili. Jak która ta co śpiewa to "Nieee Heee". Kojarzysz? No Gabryśka! "Nieee Heee”. (117) Kuwa to czekaj, to ci obszerniejszy fragment "Mam dość, nie mów mi nic, pozwól żyć, pozwól mi byyć, nie chce już..." No, słuchaj, to ja już nie mam tego pieprzyka... Taa, usunełam... Sam zszedł, bo to kleszcz był! No wiem, ja też (118) się nie brechtam, no... A jeszcze ci nie mówiłam, ze mandat ostatnio dostałam, wiesz! No wiem, że (119) prawka nie mam, ale posłuchaj jak było. Ty, (120) jechałam na balety, na stopa, wiesz, (121) zatrzymałam sie na wylotówce i (122) kuwa gibie przy trasie, co nie? Ty, ale się nikt nie chce zatrzymać, (123a) co nie, (123b) w końcu się zatrzymat koleś, ale tirem, (124) co nie! Ty, on wysiada i się mnie pyta, (125) czy mu nie pomoge towaru rozładować! (126) Wiadomo o co kaman, (127) co nie? Ty, więc mu mówię (128) no sex, ty, a on za mna ty, ja biegne, a on za mna Gabryśka, tak (129) spieprzałam, że mi fotoradar zdjecie zrobił! No, mówię ci! A Gabrycha, Gabryśka, a gdzie ty w ogóle jesteś (130) pojechana na maxiora ty krejzolko jedna, co? W kuchni? Gabrycha koniecznie idź to dużego pokoju. Do dużego pokoju idź i mi drzwi od balkonu otwórz, bo się (131) kuwa zatrzasnęłam. 


\section{(78) Papieros ${ }^{78}$}

Wir sehen auf der Bühne einen Darsteller ( $R$ - Robert), der das Publikum nach Zigaretten fragt. Gleich erscheint auf der Bühne eine Zigarette (in dieser Rolle sehen wir Igor Kwiatkowski).

R: Słuchajcie, ma ktoś może papierosa?Albo nie... Słuchajcie, chodzi o to, że ja teraz jestem $w$ takiej sytuacji obecnie, że mi ciężko o tym mówić, ponieważ ja palę i próbuję rzucić, ale mi się nie udaje, wiecie? I to jest tak z nałogiem, że ja sobie zdaje sprawe, że to jest szkodliwe i...Na przykład dzisiaj rano wstałem z taka myśle, że ani jednego więcej, słuchajcie, ani jednego więcej papierosa. Takie miałem postanowienie i nie da rady! Normalnie od rana (132) chodzi za mna papieros. Słuchajcie, ta myśl mi się pojawia i od razu... Auf der Bühne erscheint die Zigarette, gespielt von Igor Kwiatkowski

I: A kuku, cześć! Haha! Hej, hej!

R: A co ty tu robisz?

I: Co ja tu robię? No nic, wiesz, nie myśl, że cię śledzę czy coś. Po prostu zupełnie przypadkowo sobie tędy przechodze, wiesz, koło Rotundy, patrze, stoisz... podchodze, nie? Zigarette lacht, dann fängt sie an zu weinen

R: Co się dzieje?

I: Jak co się dzieje, nie udawaj! Robert, (133) ty mnie ostatnio unikasz!

R: Nie no, coś ty...

I: Co nie, nie kłam! Tylko mi tu nie kłam!

Robert, ja (134) czuję przez bibułę, że coś się święci. Co się dzieje?

R: Oj, bo (135) ja się wypaliłem chyba!

I: Wy... (136) Wypaliłem...? Ty draniu, ty... (137) Najpierw mnie podjarałeś...

R: Nie no, co ty mówisz...?

I: (138) A teraz rzucasz, jak pierwszego lepszego kiepa...!

Ty draniu, przyznaj się, (139) że chciałeś mnie tylko wykorzystać! Że chodziło ci, jak każdemu, tylko o nikotynę!

R: Słuchaj...niee!

I: Nie? Robert, no...

R: Oj, bo to (140) matka mi kazała cię rzucić!

I: Oooo... Mamunia! Cholerne babsko... Nigdy mnie nie akceptowała! Musieliśmy się chować po kiblach jak gówniarze! A pamiętasz nasza wspólna piosenke, pamiętasz, no ? Idzie rak, nieborak...

${ }^{78}$ Sketch von der Gruppe „Paranienormalni“ 
I und $R$ singen zusammen: ...masz na płucach czarny znak.

I: Ej, pamiętałeś ty kokiecie, pamiętałeś!

R: Ale tak było kiedyś!

I: Co kiedyś...

R: Teraz sobie jakoś radze, mam gumy z nikotyna!

I: Pff... Gumy z nikotyna... Weź mnie nie rozśmieszaj. (141) Nędzne substytutki!

No Robert, no! (142) Masz mnie tu jak na tacy i nic, i nic?!

No, Robert (143) rozpal mnie! Bo nie wytrzymam... Mam zapałki... No? No?!

R: No ja już nie wiem...

I: Nie wiem... Może ci mamunia powie?!

R: Ale nie płacz teraz!

I: Co nie płacz...

R: No (144) nie rozklejaj się, bo ci się tytoń wysypie, no...

I: (145) To puść dymka, badź mężczyzna!

R: Dobra, po kolacji! Tylko ani słowa matce!

I: (146a) Ha, twój pecik pragnie twoich (146b) usteczek!

R: Po kolacji mówię!

I: Dobra, dobra, dobra, znam cię, na jednym się nie skończy, (147) przyjdziemy cała paczka, ha!

\section{(79) Chińska restauracja $a^{79}$}

Auf der Bühne sehen wir einen Gast sitzen und einen Kellner, der die Bestellung aufnehmen soll. Der Kellner trägt eine traditionelle chinesische Mütze, beugt sich vor dem Gast und grüßt ihn. K-Kellner, G-Gast

K: (1) Notieti nać pan! ... Hmmm... Notieti nać pan!! Aaaaaaa.... Nasz klient nasz pan!! AAAAA... nasz klient nasz pan!! Nasz klient...

G: Dzień dobry.

K: (2) Nasz klient nasz pan!!!

G: Dobra, dobra, dobra, dobra. Co to jest?

$K:$ (3a) tuteń.

G: Przepraszam, co?

K: (3b) tuuuuteńńńńńń.

G: Przepraszam, ale ja nie rozumiem. Pytam co to jest.

K: (4) Nie rosumie, nie rosumie. Se uczy to rosumie. Oo... (3c) tuteń? miska!

${ }^{79}$ Sketch von der Gruppe „Ani Mru-Mru“ 
G: Miska?! To jest bardzo dobry żart, bardzo śmieszne, bardzo!!

K: Hahahahahaha... Dziękuje...

G: Człowieku, ja nie pytam o to... tylko o to co jest w misce.

$K:(5 a)$ Kutak w ciescie na otro.

G: Co takiego?

$\mathrm{K}$ : (5b) Kuutakk w cieescie na otro!

G: (5c)Kurczak w cieście na ostro, tak?

K: Tak!

G: A tak się składa, że (6) z waszej ulotki, napisanej niby to po Polsku, zamówiłem sobie UWAGA: (7a) kopytki ... w sosie.. na wynosi.. He!

K: (7b) My kopytki nie spsiedajemi.

G: Jak to... (7c) Co to znaczy my kopytki nie sprzedajemy? Co to w ogóle znaczy?? To dlaczego sa w waszej ulotce?!

K: (8) Chł. . Chłu. . Chłłł... Chłyt martetindodi.

G: Że... że co, proszę??

K: (8a) Hihihihi... Chłtłaaaaaa... .... Chłyt martetindodi!!!!

G: (8b) Chwyt marketingowy?!

K: Tak!!G: (8c) Proszę pana, to jest dla mnie jakiś chiński chwyt marketingowy!

K: Ale działa!

G: Może i działa. Ja też zaraz zadziałam jak nie dostanę czegoś normalnego do zjedzenia. (9) Ro-zu-miesz?

$\mathrm{K}:$ (10) Jak-sie-masz?

G: (11a) Hehehe... ping-pong?

K: (11b) Andrzej Grubba! Sto lat, sto lat!

G: Posłuchaj mnie Chińczyku. Posłuchaj mnie uważnie Chińczyku. Wszedłem tu bo byłem głodny i miałem blisko. Zamówiłem sobie jedno, dostałem zupełnie coś innego. Chce coś normalnego do jedzenia. Czy ty to rozumiesz?

K: (12)Nie wsystko. Pan młowi powolij. Ja się duszo domyślać.

G: Powoli?

K: Tak.

G: Dobrze... teraz będe mówił powoli. (13) A ty sobie weź jakiś mały chiński ołóweczek. I na malutkiej chińskiej karteczce sobie zapisz.

K: Tak.G: Tylko dokładnie.

$K:$ (14) Nasz klient nasz pan!

G: Tak, tak, tak, tak, tak... tak?

K: Tak!

G: Tak? 
K: Tak.

G: ... schabowy.

K: Tak... (15a) niadodi.

G: ... ziemniaki.

K: Tak... (15b) niotati.

G: I surówka...

K: Tak...

G: ... z czerwonej kapusty.

K: Oooo... tak! (16) Niututa so katuta... tak!

G: Tak.K: Tak.G: Tak.K: Tak.

G: Tak.

K: Tak.

G: Tak.

K: Nie ma.

G: Powtórz to!

$K:$ (17) Niiieee maaa...

G: Nie ma! To pytam po kiego grzyba bazgrolisz po tej kartce jak wiesz, że nie ma?! Skoro ci dyktuje schabowy, ziemniaki, surówka. To... (18) co to sa te czarne mróweczki, co te domki takie nastawiałeś. Co to jest $w$ ogóle. Co to jest?

K: Sajgonki...

G: SAJGONKI?!

K: (19) Ryź z wadziwami.

G: Ja widzę ryż z warzywami jak patrzę na ciebie. Wygladasz jak wielki nie ugotowany ryż z warzywami.

K: O! (20) Surówta z katusti!

G: Surówka z kapusty. Bardzo dobrze. Z jakiej? Czerwonej?

K: Pekińskiej...

G: W tym momencie moja cierpliwość się skończyła. Liczę do trzech i chce coś normalnego do jedzenia, jasne!

K: Tak.

G: Raz! Dwa! Trzy!

K: (21) Kocham cje...

G: (22) Ale ja nie potrzebuje twojej małej, skośnookiej, żółtej miłości. Tylko chce coś normalnego do jedzenia.

K: (23) Cielecina piec smaków.

G:Cielęcina pięć smaków. Dziękuje, już raz jadłem. Pół godziny szukałem mięsa między wiórkami bambusa... A kiedy już znalazłem okazało się, że to kalafior.

K: To może (24a) łebtowina....

G: Co? 
$K:(24 b)$ Łebtowina...

G: Wieprzowina?

K: Tak!

G: Wieprzowina tak? A ile smaków 17 czy 61??

K: Niee...G: Ja nie lubię chińskiej kuchni... wszedłem tu bo miałem blisko... (25). z chińskich rzeczy to lubię tylko podkoszulki... Zajrzyj do ulotki i znajdź mi coś normalnego do zjedzenia... to jest twoja ostatnia szansa...

K: Tak...

G: ... ostatnia szansa w życiu...

K: No to, no to może (26a) dzupa...??

G: Ale, że co (26b) dzupa?

K: (26c ) Chińska dzupa... NIE!!! Nie o to chodzi... dzupa chińska...

G: Zupa?!

K: Tak... (26d) dzupa...

G: Zupa.

$K:(26 e)$ dzupa.

G: Zu-

K: (26f) Dzu-

$G$ und K: (26g) -pa...

G: Ale chińska nie! Nie jadłem chińskiej, nie jem i nigdy nie będe jej jeść...

$\mathrm{K}:$ Ale... ale...

G: Stop! Skończyłem dyskusję w tym momencie!! Nigdy nie...

K: Ale (26h) dzupa chińska, ale robiona w Radomiu! W ogóle nie smakuje jak chińska!

G: W takim razie moge zaryzykować. Tylko raz-raz. No! Jak jest po chińsku raz-raz?

K: (27a) Ekekmewuzudu.

G: No (27b) to wuzudu, bo jestem głodny, jasne?

K: Tak.

G: A z czego ta zupa?

K: Nie wiem, nie napisano po chińsku na opakowanniu.

G: Pewnie z psa.

K: O nie, nie, nie. My z psa robimy cieleccine.

G: To aż boje się spytać co robicie z kota.

$K: A$ z kota to kisiel i ciastka.

G: ?? A długo jeszcze będę czekał?

K: (28) Tsy minuty!! Tsy minuty. Tsy...

G: To jakieś chińskie trzy minuty, chyba. 
K: (29) Już lece, pedze... dobra dzupa dobra... hehe... my presles... Aaaaaaaa... O kutwa!!

G: (30) I co narobiłeś kitajcu?! Co narobiłeś kitajcu?! Nie dość, że jestem głodny, zły to jeszcze oblewasz mnie ta cholerna zupa z Radomia! Ty, ty, ty, ty....

K: (31a) Hutank-tank-ze.

G: (31b) Hutanktankze?! A co to znaczy po chińsku?? (32a) Pan niescięscie?? Od dzisiaj będe tu przychodził codziennie, i ty codziennie bedziesz mi robił suszi, aż całe spodnie wysuszi! Jasne!? Aha... i jeszcze jedno... (32b) zmień nazwe baru na niescięscie! (32c) Obsługa: pan niescięscie...(32d) Specjalność zakładu: niescięscie... K: (32e) Tak... ja nietęcie! (33) Klient dzisiaj wielkie scięscie wielkie... (34) strach pomyśleć co by było, gdyby dzupa była goraca

(80) Werbung für "Turk-Deutsch-Simulator"

Kennen Sie das? Niemand versteht Sie, keiner weiß, was Sie wollen... und das alles, weil Sie die Sprache Ihres Heimatlandes nicht beherrschen.

Mit dem Turk-Deutsch-Simulator versteht man Sie auch in Deutschland wieder. Richtig angewendet, verwandelt der TurkDeutsch-Simulator Ihr verstaubtes Hochdeutsch in lupenreines Asi-Turk-Deutsch.

\section{Verzeichnis der besprochenen Sketchtexte}

(14) Polizeikontrolle, S. 25

(49) Advents- und Weihnachtszeit in Deutschland, S. 68

(50) Bedienungsanleitung, S. 71

(51) Hakan und Geburtsvorbereitung, S. 73

(52) Umfrage auf der Straße, S. 74

(53) Türken und Polizeikontrolle,S. 75

(54) Ranjid und Kayas Mütze, S. 76

(55) Ranjid im SAT.1-Studio, S. 77

(56) Deutscher Integrationstest, S. 77

(57) Lustige Post, S. 79

(58) Radio Süleyman, S. 79

(59) Passantenbefragung, S. 81

(60) Szene im Zimmer von Kaya Yanar, S. 86 
(61) Szene auf dem Polizeirevier, S. 87

(62) Szene im "Radio Süleyman”, S. 88

(63) Ein Ausländer befragt die Passanten, S. 89

(64) Türkischer Dolmetscher beim Bewerbungsgespräch, S. 91

(65) Werbung für Dreier-Fahrt, S. 93

(66) Polizeikontrolle 1, S. 94

(67) Fahrschule Kelal Yildirim, S. 95

(68) Francesco im Restaurant 1, S. 96

(69) Francesco im Restaurant2, S. 96

(70) Francesco im Restaurant3, S. 96

(71) Francesco im Restaurant4, S. 97

(72) Tarek ab del Kalek auf der Modemesse in Düsseldorf 1., S. 98

(73) Tarek ab del Kalek auf der Modemesse in Düsseldorf 2., S. 99

(74) Schachweltmeisterschaften, S. 100

(75) M jak młotek ${ }^{80}$, S. 107

(76) Mariolka $1^{81}$, S. 111

(77) Mariolka 282, S. 115

(78) Papieros ${ }^{83}$, S. 119

(79) Chińska restauracja ${ }^{84}$, S. 124

(80) Werbung für "Turk-Deutsch-Simulator"85, S. 135

\footnotetext{
${ }^{80}$ Sketch von der Gruppe „Paranienormalni““

${ }^{81}$ Sketch von der Gruppe „Paranienormalni“

82 Sketch von der Gruppe „Paranienormalni“

${ }^{83}$ Sketch von der Gruppe „Paranienormalni“

${ }^{84}$ Sketch von der Gruppe „Ani Mru-Mru“

${ }^{85}$ Aus ,Was guckst du?“"
} 


\section{VON DER REDAKTION}

Dr. Katarzyna Sikorska-Bujnowicz:

- Studium an der Germanistik der Universität Łódź (1987-1992) und an der Humboldt Universität Berlin (1989-1990).

- Stipendienaufenthalte und Forschungsstipendien: Universität Bochum (02.1995-08.1995); Justus-Liebig-Universität Giessen (1995, 1996); DAAD-Jahresstipendium an der Justus-Liebig-Universität Giessen (1998-1999).

- Seit 1992 als wissenschaftlicher Mitarbeiter an der Universität Łódź tätig: in den Jahren 1992-2001 - wissenschaftlicher Assistent am Lehrstuhl für Deutsche und Angewandte Sprachwissenschaft; seit 2001 - Oberassistent am Lehrstuhl für Deutsche und Angewandte Sprachwissenschaft (Abteilung für Deutsche Sprachwissenschaft)

- Didaktische Schwerpunkte: beschreibende Grammatik der deutschen Sprache; Syntax der deutschen Sprache; kontrastive Grammatik; praktische Grammatik der deutschen Sprache; Sprachpraxis; konfrontative Forschungen im Bereich der Lexikologie

- Forschungsschwerpunkte: lexikalische Elemente fremder Herkunft im Gegenwartspolnischen und im Gegenwartsdeutschen; konfrontative Forschungen im Bereich der Lexikologie; sprachliche und außersprachliche Mittel des Humoristischen in der Konfrontation Deutsch vs. Polnisch 